\title{
Physical, Chemical, and Mineralogical Data from Surficial Deposits, Groundwater Levels, and Water Composition in the Area of Franklin Lake Playa and Ash Meadows, California and Nevada
}
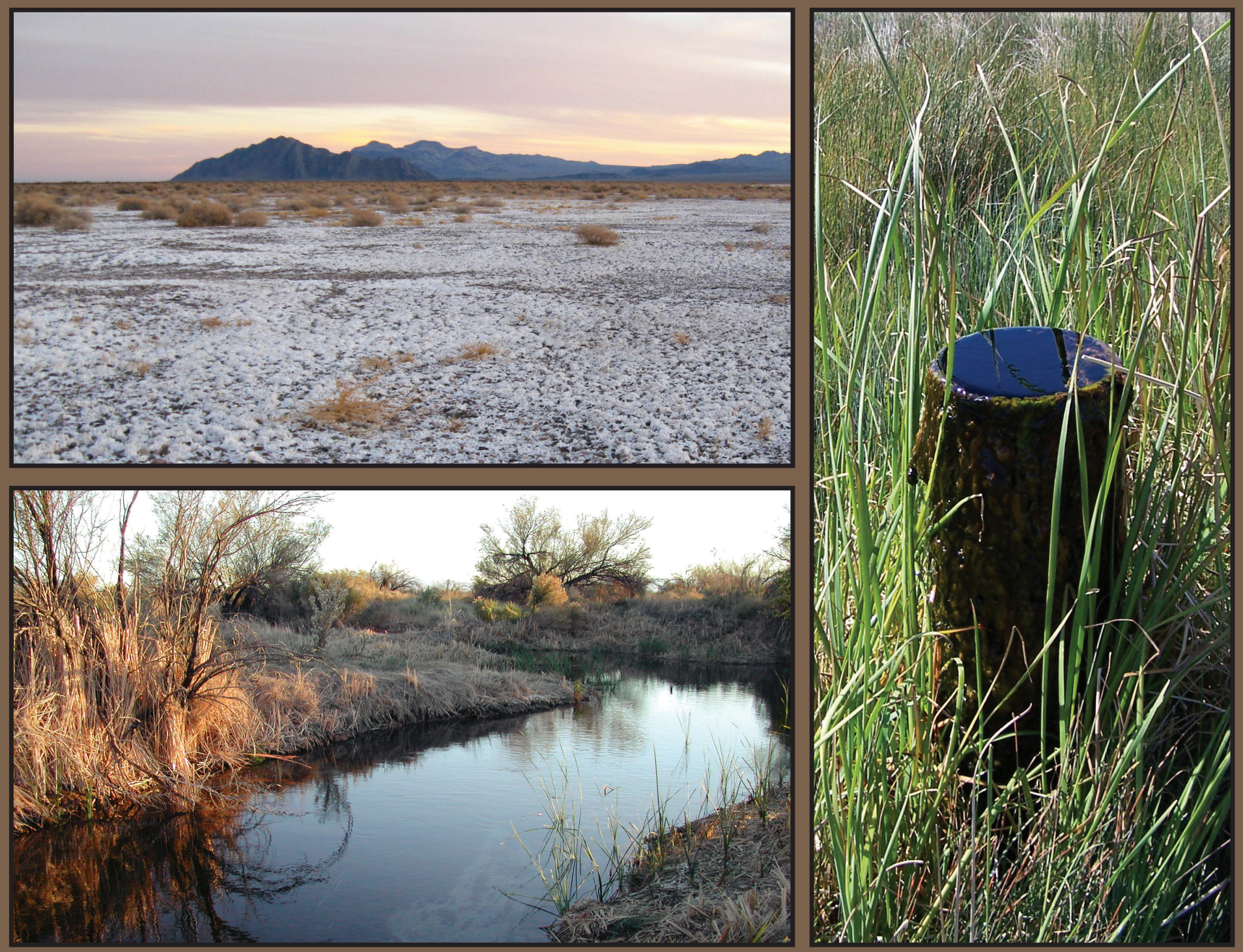

Data Series 607

U.S. Department of the Interior U.S. Geological Survey 


\section{FRONT COVER}

Upper Left: View south from site FP-CSL in Carson Slough showing recently precipitated salt crusts. Eagle Mountain in distance. Photograph by Richard L. Reynolds.

Lower left: Spring discharge from Crystal Spring at Ash Meadows National Wildlife Rufuge. Photograph by Richard L. Reynolds.

Right: Obelisk artesian well. Photograph by Harland L. Goldstein. 


\section{Physical, Chemical, and Mineralogical Data from Surficial Deposits, Groundwater Levels, and Water Composition in the Area of Franklin Lake Playa and Ash Meadows, California and Nevada}

By Harland L. Goldstein, George N. Breit, James C. Yount, Richard L. Reynolds, Marith C. Reheis, Gary L. Skipp, Eric M. Fisher, and Paul J. Lamothe

Data Series 607 


\title{
U.S. Department of the Interior \\ KEN SALAZAR, Secretary \\ U.S. Geological Survey \\ Marcia K. McNutt, Director
}

\author{
U.S. Geological Survey, Reston, Virginia: 2011
}

For more information on the USGS - the Federal source for science about the Earth, its natural and living resources, natural hazards, and the environment, visit http://www.usgs.gov or call 1-888-ASK-USGS.

For an overview of USGS information products, including maps, imagery, and publications, visit http://www.usgs.gov/pubprod

To order this and other USGS information products, visit http://store.usgs.gov

Any use of trade, product, or firm names is for descriptive purposes only and does not imply endorsement by the U.S. Government.

Although this report is in the public domain, permission must be secured from the individual copyright owners to reproduce any copyrighted materials contained within this report.

Suggested citation:

Goldstein, H.L., Breit, G.N., Yount, J.C., Reynolds, R.L., Reheis, M.C., Skipp, G.L., Fisher, E.M., and Lamothe, P.J., 2011, Physical, chemical, and mineralogical data from surficial deposits, groundwater levels, and water composition in the area of Franklin Lake playa and Ash Meadows, California and Nevada: U.S. Geological Survey Data Series 607, 153 p. 


\section{Contents}

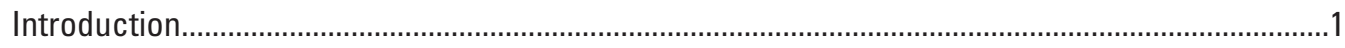

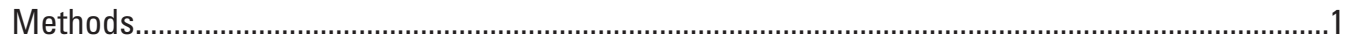

Site Selection and Sampling Locations ...................................................................................

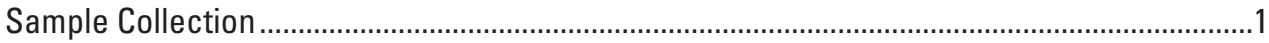

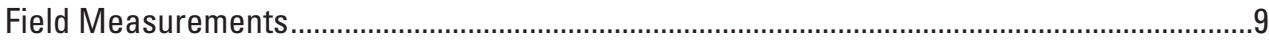

Surface Characteristics....................................................................................................

Groundwater Levels, Well Data, and Field Parameters ...................................................10

Laboratory Analysis.............................................................................................................. 10

Gravimetric Water Content ....................................................................................... 10

Carbonate Percent ........................................................................................................... 10

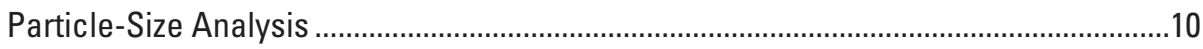

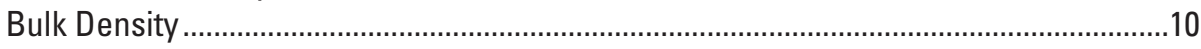

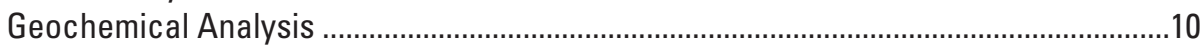

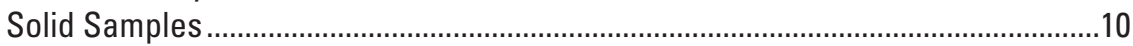

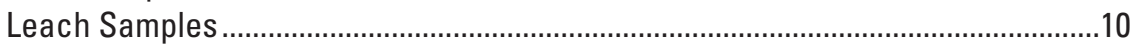

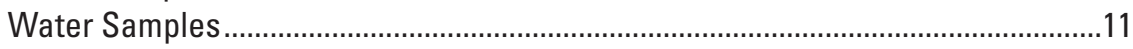

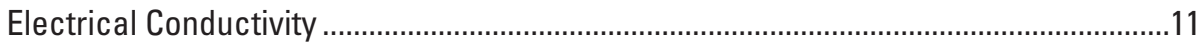

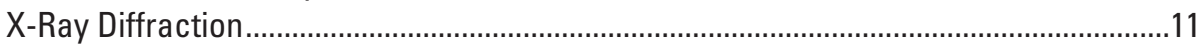

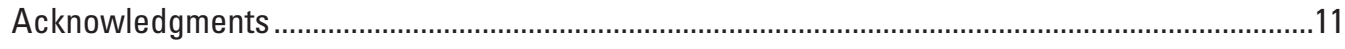

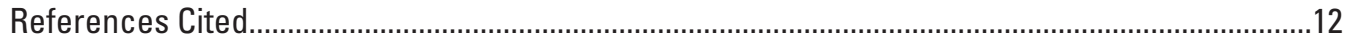

\section{Figures}

1A. Shaded relief map showing regional setting of the Franklin Lake playa and Ash Meadows study area ..............................................................................................

1B. Satellite image showing location of Franklin Lake playa and Ash Meadows

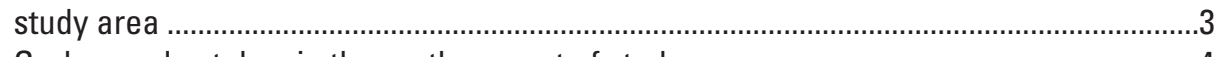

1C. Grab samples taken in the northern part of study area .....................................................

1D. Grab samples taken in the central part of study area .......................................................5

1E. Auger and grab samples taken in the southern part of study area..................................6

1F. Repeat and dust trap sites ..........................................................................................

1G. Water samples taken throughout study area ..............................................................

\section{Tables}

1. Location information for sampling sites...........................................................................14

2. Summary of laboratory analyses completed on sediment samples .................................17

3. Summary of field and laboratory water analyses completed ..........................................25

$4 A-J$. Repeat measurement data for:

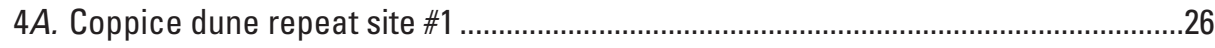

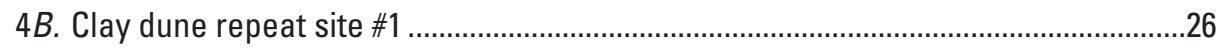

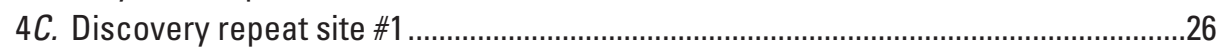

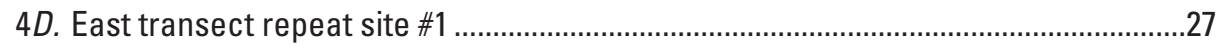

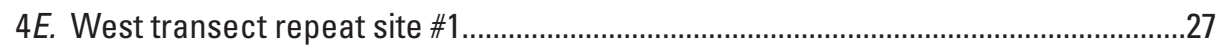

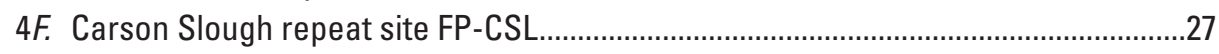

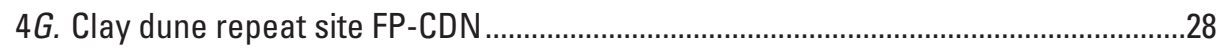

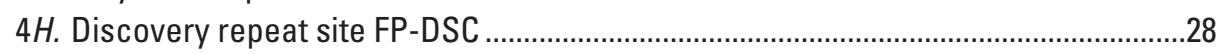

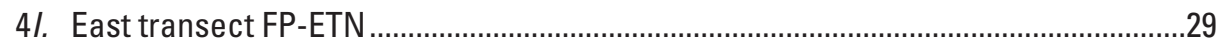

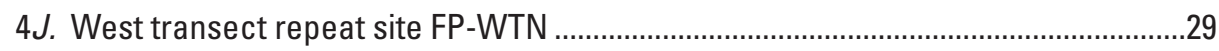


5. Casing, water level, well depth, and ground-surface elevations for Franklin Lake playa wells

6. Values of field parameters measured on water samples ...................................................34

7. Moisture content of sediment collected by augering at Franklin Lake playa......................35

8. Carbonate percent of selected grab samples .................................................................38

9A-C. Particle size data for:

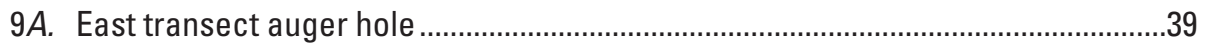

9B. Coppice dune site auger hole ................................................................................ 43

9C. Franklin Lake playa and Ash Meadows grab samples...............................................46

10. Bulk density data of Franklin Lake playa grab samples ....................................................53

11A-C. Geochemical data determined on:

11A. Selected bulk East transect auger hole samples .................................................54

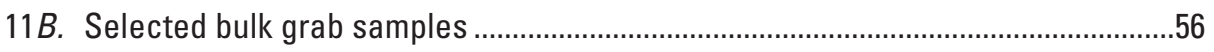

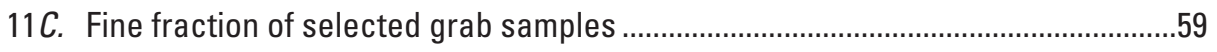

12A-I. Composition of:

12A. DI-leach bulk East transect auger hole samples ...............................................60

12B. Nitric acid leach bulk East transect auger hole samples......................................66

12C. Residual fraction of bulk East transect auger hole samples .................................72

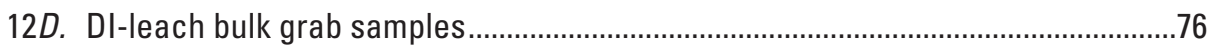

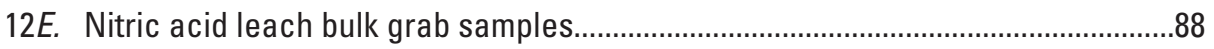

12F. Residual fraction of bulk grab samples ................................................................102

12G. DI-leach fine fraction of grab samples .................................................................110

$12 \mathrm{H}$. Nitric acid leach fine fraction of grab samples.....................................................117

121. Residual fraction of fine fraction of grab samples ..............................................124

13. Composition of water samples ......................................................................................... 129

$14 A-B$. Electrical conductivity measurements of:

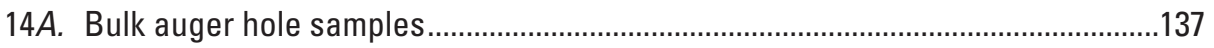

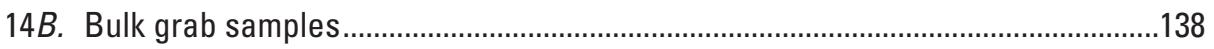

15. Mineralogical data of auger hole and grab samples determined by

X-ray diffraction 


\section{Conversion Factors}

SI to Inch/Pound

\begin{tabular}{lcl}
\hline Multiply & By & To obtain \\
\hline centimeter $(\mathrm{cm})$ & Length & \\
millimeter $(\mathrm{mm})$ & 0.3937 & inch (in.) \\
meter $(\mathrm{m})$ & 0.03937 & inch (in.) \\
kilometer $(\mathrm{km})$ & 3.281 & foot (ft) \\
kilometer $(\mathrm{km})$ & 0.6214 & mile (mi) \\
meter $(\mathrm{m})$ & 0.5400 & mile, nautical (nmi) \\
\hline & 1.094 & yard (yd) \\
\hline square meter $\left(\mathrm{m}^{2}\right)$ & Area & \\
square centimeter $\left(\mathrm{cm}^{2}\right)$ & 0.0002471 & acre \\
square meter $\left(\mathrm{m}^{2}\right)$ & 0.001076 & square foot $\left(\mathrm{ft}^{2}\right)$ \\
square centimeter $\left(\mathrm{cm}^{2}\right)$ & 10.76 & square foot $\left(\mathrm{ft}^{2}\right)$ \\
\hline & 0.1550 & square inch (ft $\left.{ }^{2}\right)$ \\
\hline liter $(\mathrm{L})$ & Volume & \\
liter $(\mathrm{L})$ & 33.82 & ounce, fluid (fl. oz) \\
liter $(\mathrm{L})$ & 2.113 & pint (pt) \\
liter $(\mathrm{L})$ & 1.057 & quart (qt) \\
cubic meter $\left(\mathrm{m}^{3}\right)$ & 0.2642 & gallon (gal) \\
cubic meter $\left(\mathrm{m}^{3}\right)$ & 264.2 & gallon (gal) \\
cubic centimeter $\left(\mathrm{cm}^{3}\right)$ & 0.0002642 & million gallons $(\mathrm{Mgal})$ \\
liter $(\mathrm{L})$ & 0.06102 & cubic inch (in $\left.{ }^{3}\right)$ \\
\hline gram $(\mathrm{g})$ & 61.02 & cubic inch (in $\left.{ }^{3}\right)$ \\
kilogram $(\mathrm{kg})$ & Mass & \\
\hline & 0.03527 & ounce, avoirdupois $(\mathrm{oz})$ \\
\hline kilopascal $(\mathrm{kPa})$ & 2.205 & pound avoirdupois $(\mathrm{lb})$ \\
\hline gram per cubic centimeter $\left(\mathrm{g} / \mathrm{cm}^{3}\right)$ & Pressure & \\
\hline
\end{tabular}

Temperature in degrees Celsius $\left({ }^{\circ} \mathrm{C}\right)$ may be converted to degrees Fahrenheit $\left({ }^{\circ} \mathrm{F}\right)$ as follows: ${ }^{\circ} \mathrm{F}=\left(1.8 x^{\circ} \mathrm{C}\right)+32$

Temperature in degrees Fahrenheit $\left({ }^{\circ} \mathrm{F}\right)$ may be converted to degrees Celsius $\left({ }^{\circ} \mathrm{C}\right)$ as follows: ${ }^{\circ} \mathrm{C}=\left({ }^{\circ} \mathrm{F}-32\right) / 1.8$

Vertical coordinate information is referenced to the insert datum name North American Vertical Datum of 1988 (NAVD 88).

Horizontal coordinate information is referenced to the insert datum name North American Datum of 1983 (NAD 83).

Altitude, as used in this report, refers to distance above the vertical datum.

Specific conductance is given in millisiemens per centimeter at 25 degrees Celsius $(\mu \mathrm{S} / \mathrm{cm}$ at $\left.25^{\circ} \mathrm{C}\right)$.

Concentrations of chemical constituents in water are given either in milligrams per liter ( $\mathrm{mg} / \mathrm{L}$ ) or micrograms per liter ( $\mu \mathrm{g} / \mathrm{L})$.

NOTE TO USGS USERS: Use of liter (L) as a special name for cubic decimeter $\left(\mathrm{dm}^{3}\right)$ is restricted to the measurement of liquids and gases. No prefix other than milli should be used with liter. Metric ton ( $\mathrm{t}$ ) as a name for megagram ( $\mathrm{Mg}$ ) should be restricted to commercial usage, and no prefixes should be used with it. 



\title{
Physical, Chemical, and Mineralogical Data from Surficial Deposits, Groundwater Levels, and Water Composition in the Area of Franklin Lake Playa and Ash Meadows, California and Nevada
}

\author{
By Harland L. Goldstein, George N. Breit, James C. Yount, Richard L. Reynolds, Marith C. Reheis, Gary L. Skipp, \\ Eric M. Fisher, and Paul J. Lamothe
}

\section{Introduction}

This report presents data and describes the methods used to determine the physical attributes, as well as the chemical and mineralogical composition of surficial deposits; groundwater levels; and water composition in the area of Franklin Lake playa and Ash Meadows, California and Nevada (figs. $1 A$ and $1 B$ ).

The results support studies that examine (1) the interaction between groundwater and the ground surface, and the transport of solutes through the unsaturated zone; (2) the potential for the accumulation of metals and metalloids in surface crusts; (3) emission of dust from metal-rich salt crust; and (4) the effects of metal-rich dusts on human and ecosystem health.

The evaporation of shallow ( $<3$ to $4 \mathrm{~m}$ ) groundwater in saline, arid environments commonly results in the accumulation of salt in the subsurface and (or) the formation of salt crusts at the ground surface. Ground-surface characteristics such as hardness, electrical conductivity, and mineralogy depend on the types and forms of these salt crusts. In the study area, salt crusts range from hard and bedded to soft and loose (Reynolds and others, 2009). Depending on various factors such as the depth and composition of groundwater and sediment characteristics of the unsaturated zone, salt crusts may accumulate relatively high contents of trace elements (Breit and others, 2009).

Soft, loose salt crusts are highly vulnerable to wind erosion and transport. These vulnerable crusts, which may contain high contents of potentially toxic trace elements, can travel as atmospheric dust and affect human and ecosystem health at local to regional scales (Reynolds and others, 2007).

\section{Methods}

\section{Site Selection and Sampling Locations}

The study area encompasses Franklin Lake playa (FLP) and portions of Ash Meadows National Wildlife Refuge (fig. 1B). Information about sampling sites is displayed in table 1. Surficial materials at the study sites were studied by repeated in situ measurements, and samples were collected for laboratory determination of physical, chemical, and mineralogical properties (table 2). Water levels in wells were recorded and samples were collected for geochemical characterization (table 3).

Sediment sampling sites were chosen to be representative of the spatial variations across the study area. Although the study area is temporally dynamic, it can be divided into seven general areas on the basis of overall persistent surface characteristics: (1) spring discharge areas, (2) salt pans, (3) areas with porous and sometimes soft sediments, (4) hard and compact areas, (5) areas dominated by braided stream channels, (6) areas dominated by coppice dunes, and (7) vegetated, desert pavement surfaces (table 1; Czarnecki, 1997). In the Ash Meadows area, surface and subsurface sediment samples were collected along the Carson Slough in the west-central part of the refuge, and near Rogers Spring in the north (fig. 1C). Sediments collected from FLP include surface and subsurface samples and samples collected by hand auger to depths as much as $4.3 \mathrm{~m}$ (figs. $1 D$ and $1 E$ ). Some FLP sample sites, referred to as repeat sites, were identified for replicate sampling (fig. $1 F$; discovery, coppice dune, west transect, east transect, clay dune, and salt pan).

Existing wells and springs were used for the study of groundwater in the area. Water levels were determined during many of the field visits. Water samples were collected from spring discharge pools at Ash Meadows and from artesian and nonflowing wells at FLP (fig. $1 G$ ).

\section{Sample Collection}

Sediment samples were collected by grab sampling and with a hand auger. Auger samples were collected from each bucket retrieved, typically representing $<15-\mathrm{cm}$ intervals. Samples from discrete intervals, referred to as grab samples, were collected from small excavations using a hand trowel or shovel. At these sites a surface-crust sample (usually the upper $\approx 1 \mathrm{~cm}$ ) and two subsurface samples (on the order of 1-5 cm and 5-10 cm depths) were collected. Surface measurements were made at repeat sites, and surface-crust grab samples were collected for mineralogic identification intermittently for approximately 2.5 years. 


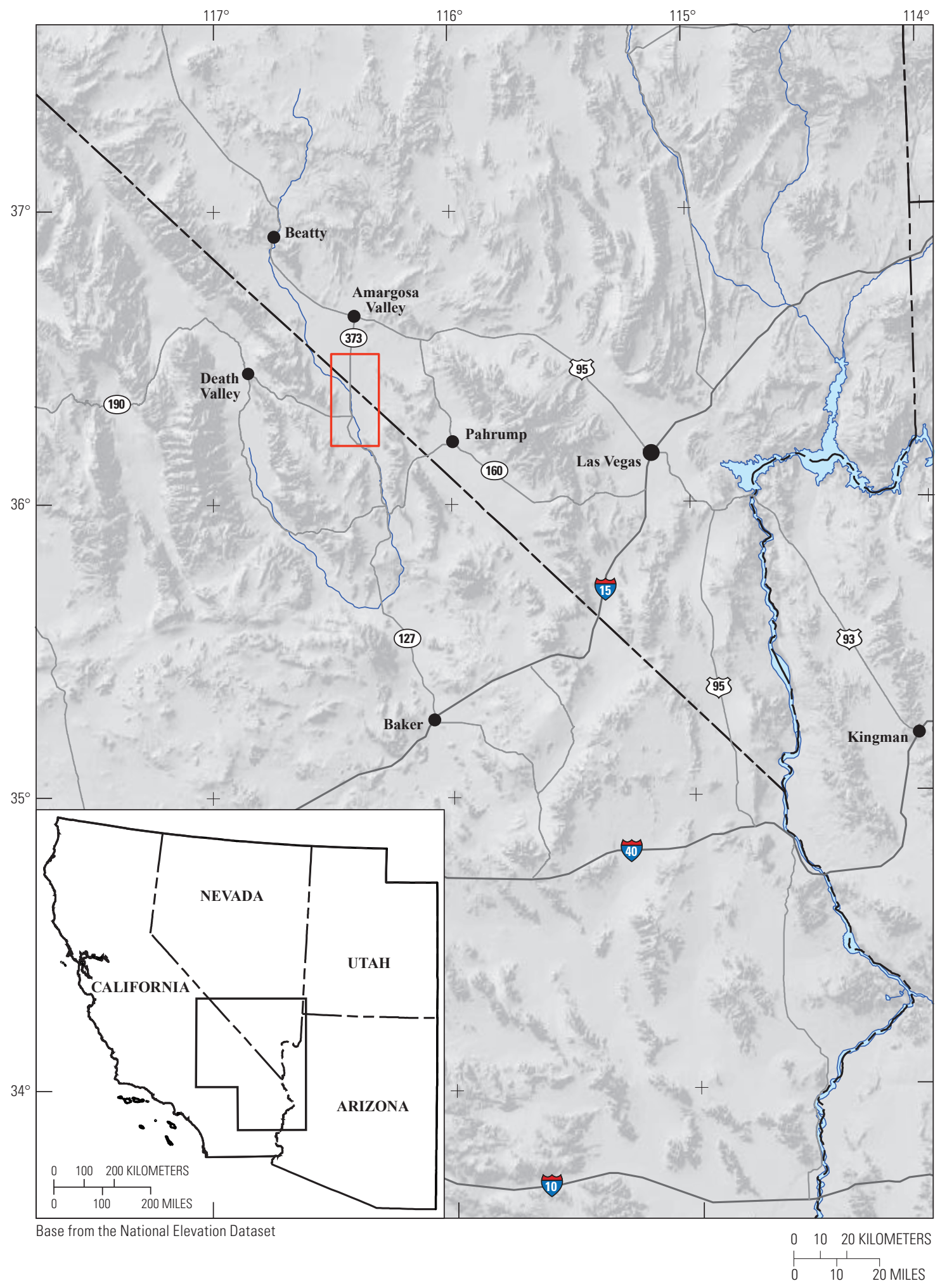

Figure 1A. Shaded relief map showing regional setting of the Franklin Lake playa and Ash Meadows study area. The study area (and the extent of figure $1 B$ ) is noted by red box. Inset index map shows coverage of the shaded relief map. 


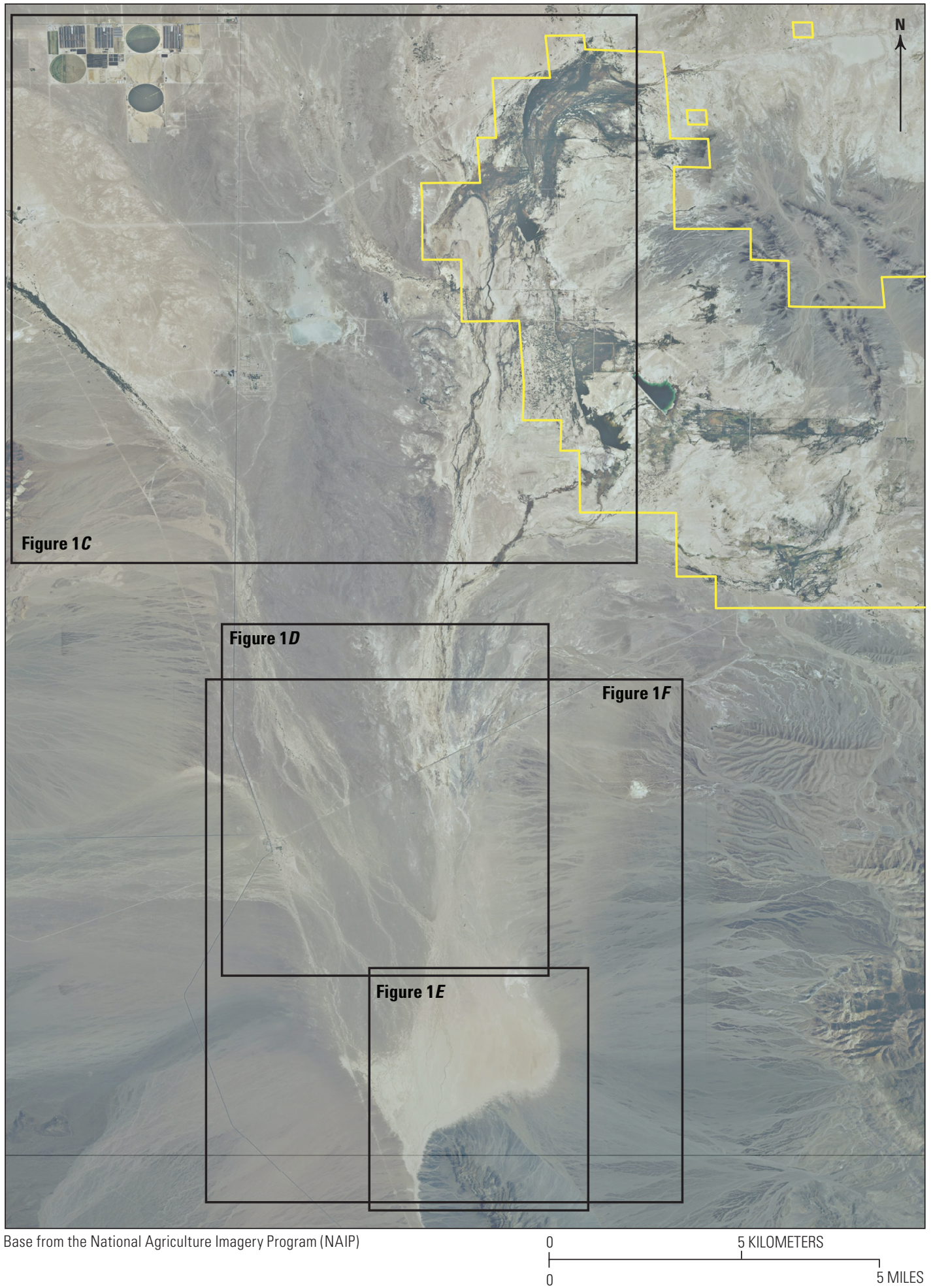

Figure 1B. Satellite image showing the Franklin Lake playa and Ash Meadows study area. Black boxes denote area covered in figures $1 \mathcal{C}-1$. Yellow line represents the Ash Meadows National Wildlife Refuge boundary. 


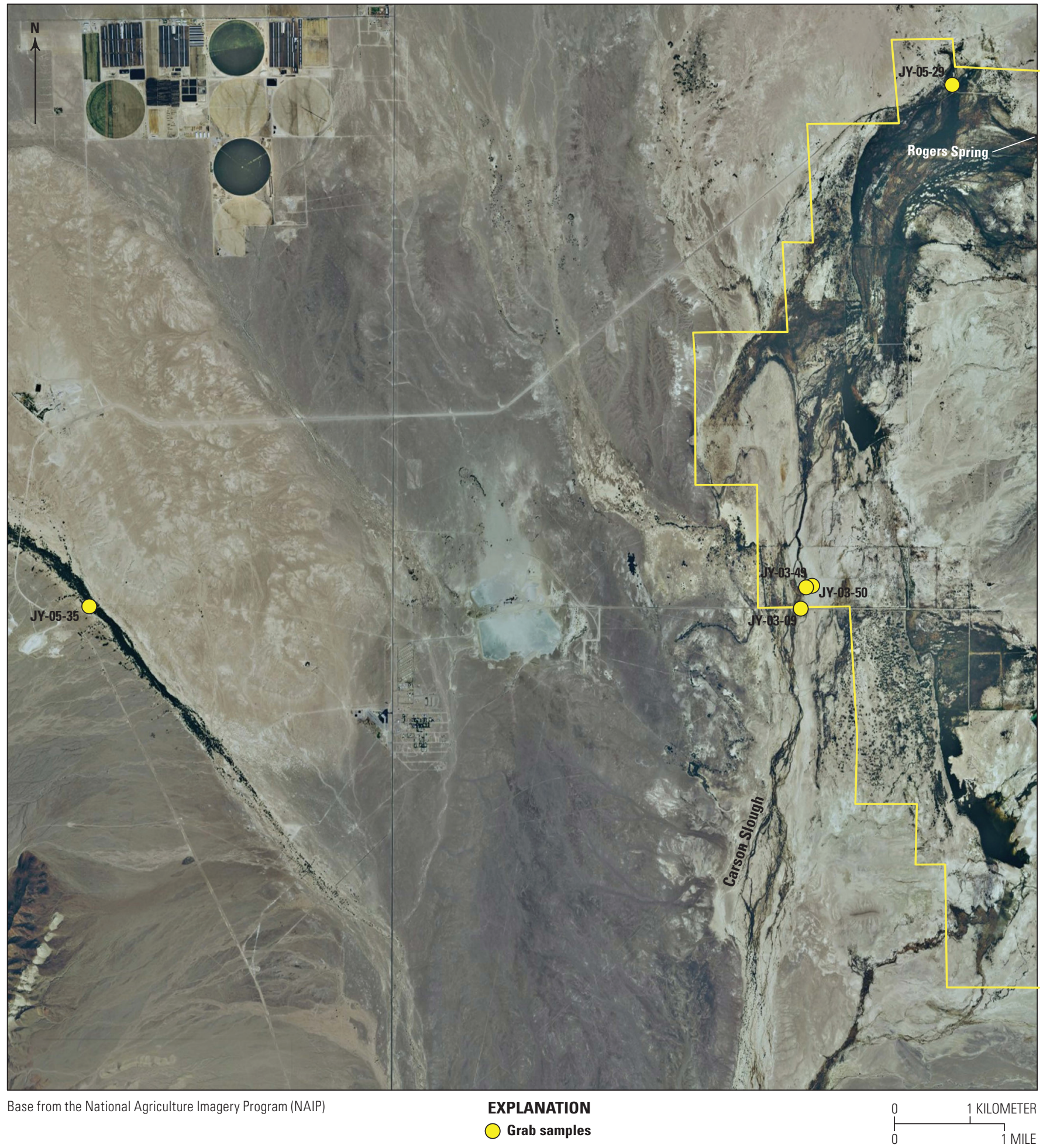

Figure 1C. Location of grab samples taken in the northern part of study area. Yellow line represents the Ash Meadows National Wildlife Refuge boundary. 


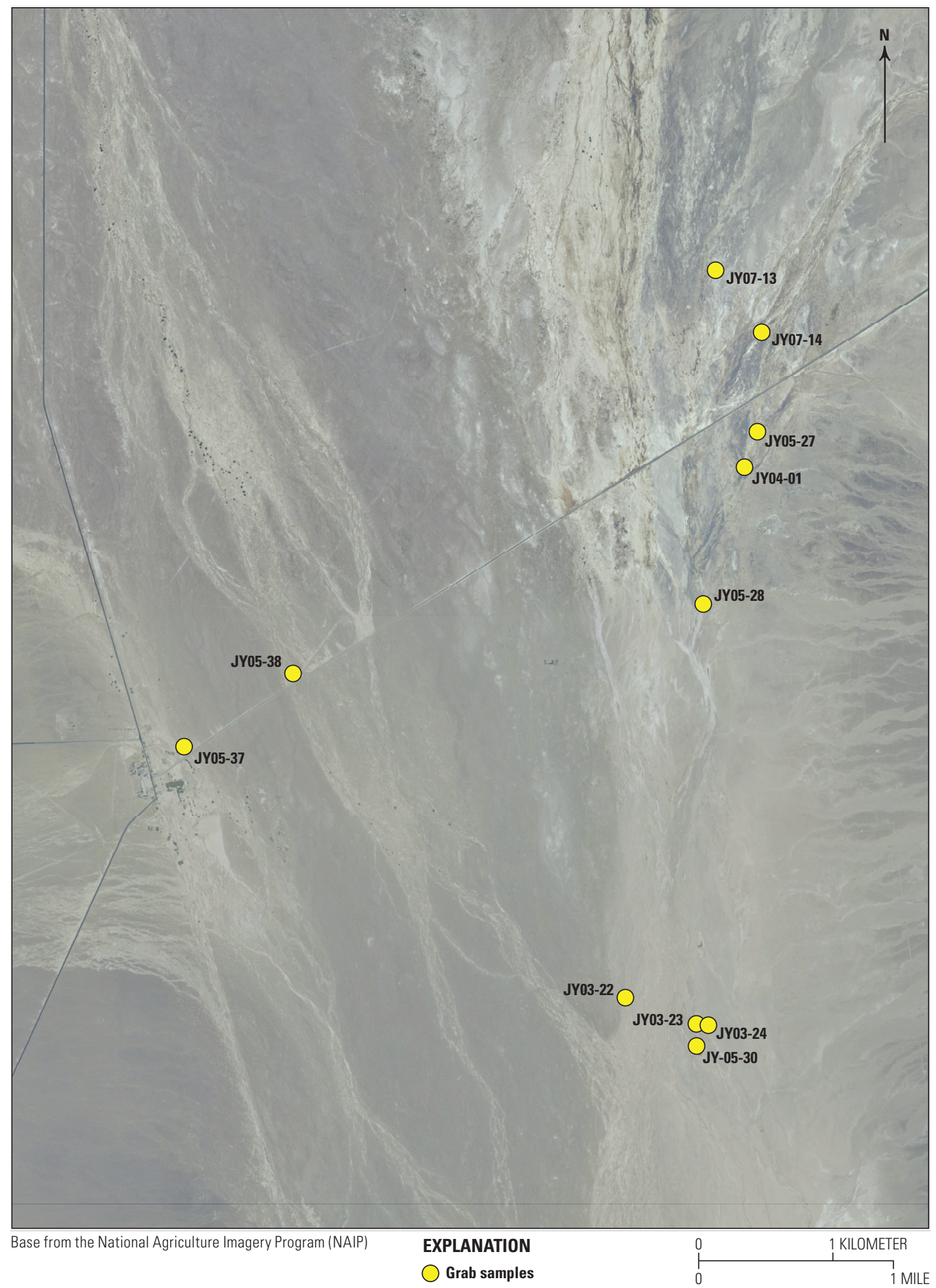

Figure 1D. Location of grab samples taken in the central part of study area. 


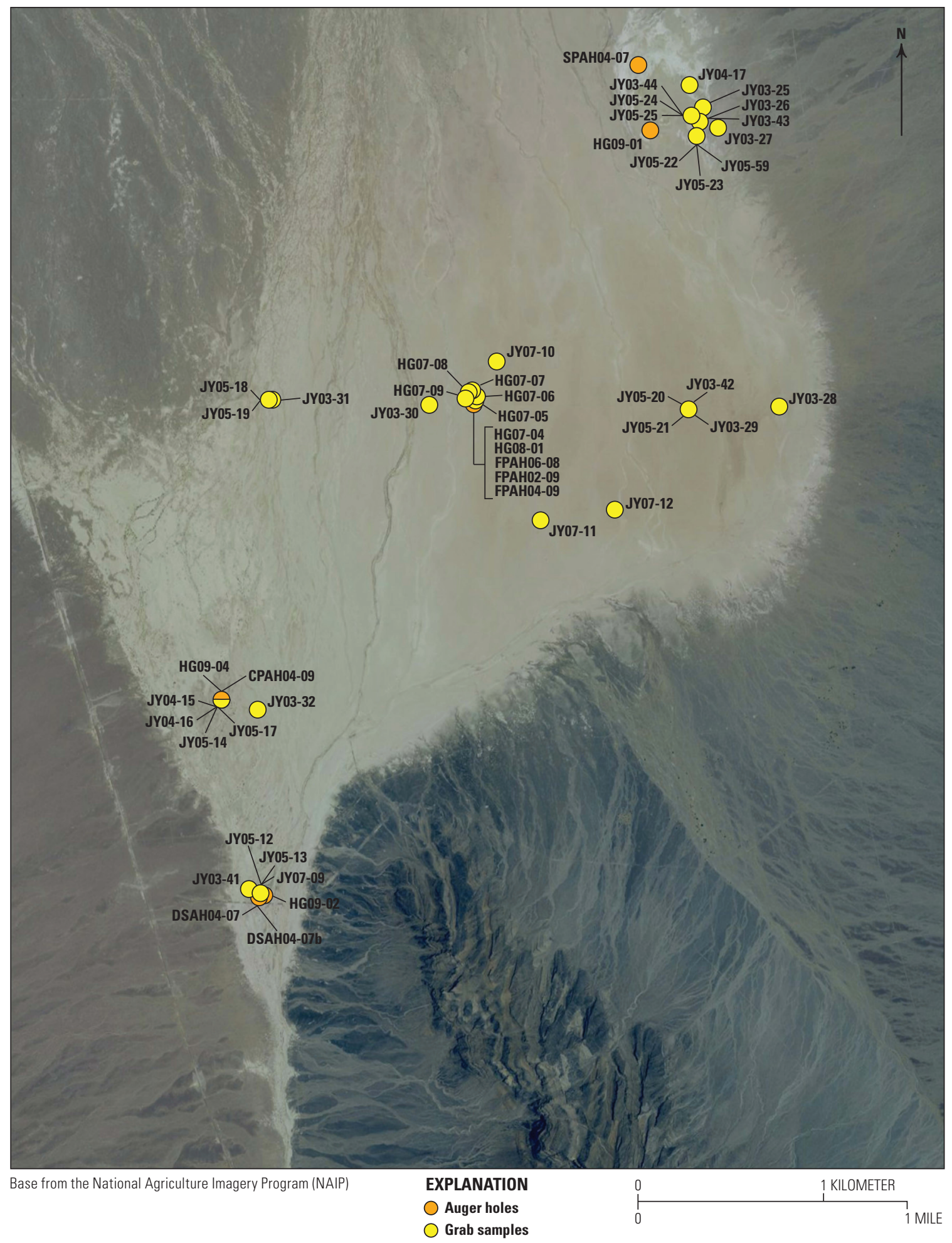

Figure 1E. Location of auger and grab samples taken in the southern part of study area. 


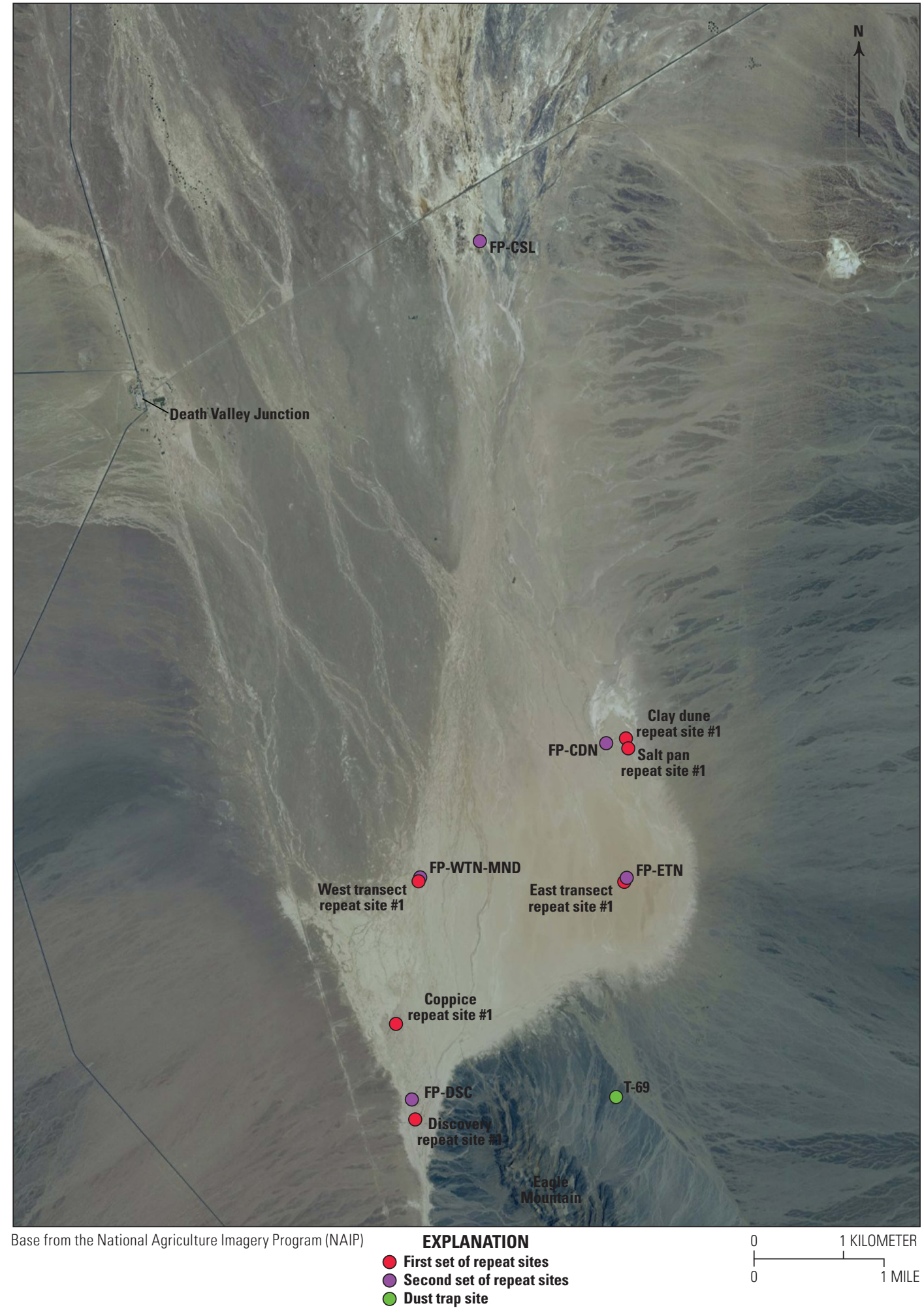

Figure 1F. Location of repeat and dust trap sites. 


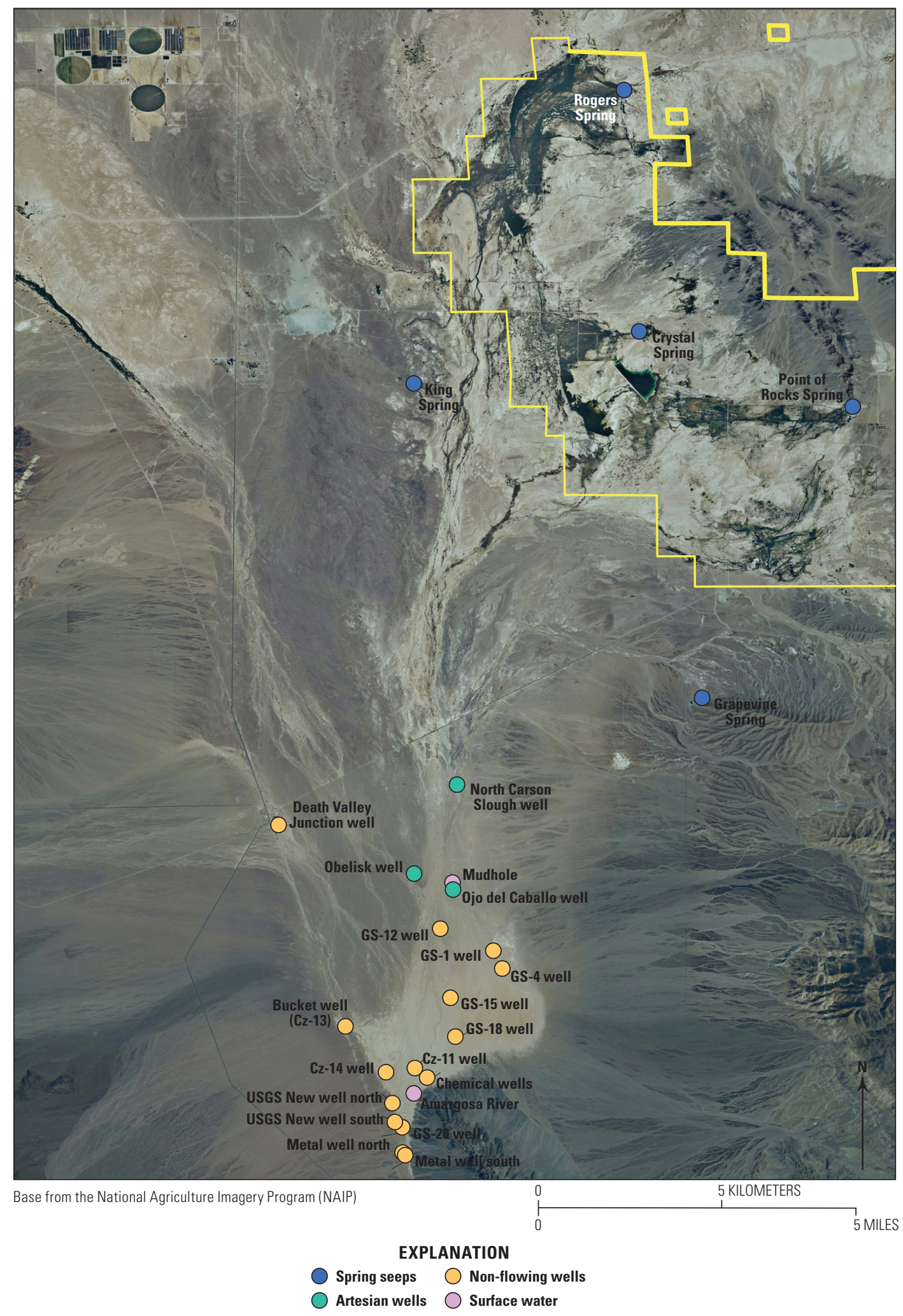

Figure 1G. Location of water samples taken throughout study area. Yellow line represents the Ash Meadows National Wildlife Refuge boundary. 
Marble dust traps (Reheis and Kihl, 1995; Reheis, 1997) were installed in February 2004 on the southern end of FLP on an alluvial fan surface on the northeastern flank of Eagle Mountain (fig. $1 F$ ). Dust samples were collected during the spring and fall of subsequent years until the dust traps were removed in November 2009. Dust analyses are not included in this report except for one dust trap sample that was collected in the spring of 2005. This sample represents the collection period of October 2004 through April 2005 and was leached for chemical analysis (see below).

Methods to collect water samples varied depending on characteristics of the sample site. Spring seeps were sampled directly by drawing water into a clean $60-\mathrm{mL}$ syringe, or for large pools (Crystal and Rogers Springs), through tubing connected to a peristaltic pump. The tubing was suspended over the pools with a pole and lowered to a depth of approximately $10 \mathrm{~cm}$ below the pool surface prior to sampling. Water from flowing wells (North Carson Slough, Ojo del Caballo, and Obelisk wells) was collected from tubing that was lowered approximately $1 \mathrm{~m}$ into the well casing. Several wells installed by Czarnecki (1997) (GS-4, GS-12, GS-15, GS-18, GS-20) were pumped until empty and allowed to refill for 4 to 6 hours prior to sampling. A peristaltic pump was used to purge and sample these wells. A recently installed well (USGS New well north) was pumped to remove about $100 \mathrm{~L}$ of water and then sampled. The small amount of suspended clay in the effluent suggests that this well was incompletely purged at the time of sampling. The Death Valley Junction well was an actively used supply well with an electric pump. Water at this site was collected from a petcock valve installed on the production pipe. Three sample sites (Bucket, Chemical, and Cz-11 wells) were large diameter wells that were incompletely purged prior to sampling. The Bucket well was sampled with a peristaltic pump after removal of approximately $25 \mathrm{~L}$ of water. A bailer was used to sample the Chemical well after drawing approximately $30 \mathrm{~L}$ of water from the well bore. $\mathrm{Cz}-11$ well was pumped to remove the standing water, leaving a muddy slurry that was collected in a beaker and allowed to settle, before collecting the water fraction. The Chemical and Cz-11 wells were open at the surface; therefore, atmospheric input may influence these water compositions.

One surface-water sample was collected from the Amargosa River at the southern end of FLP shortly after a heavy flood event. The sample was collected from a flowing channel into a plastic bottle without filtration or stabilization.

Water samples were collected for anion, cation, trace element, and isotopic analyses. All water samples were filtered to $0.2 \mathrm{~mm}$ with a syringe filter. Sampled water rinsed the syringe, filter, and collection bottles prior to obtaining the sample for analysis. Anion samples were stored with ice in deionizedwater-rinsed polyethylene bottles. Water samples for cation and trace element analyses were collected in acid-washed polyethylene bottles and acidified in the field to $\mathrm{pH}<2$ with high-purity nitric acid. A separate aliquot of water was acidified to a $\mathrm{pH}$ between 3 and 4 with hydrochloric acid for preservation of dissolved sulfate to be used for isotopic analysis.

\section{Field Measurements}

\section{Surface Characteristics}

Surface characteristics were measured at two sets of repeat sites (fig. $1 F$ ). The first set of sites was established during the initial visits to the area. The sites were visited irregularly from August 2003 through April 2007 (tables 4A, B, C, D, and E). These sites consisted of a triangular plot area of approximately $45 \mathrm{~m}^{2}$ with a rebar stake at each apex. The rebar was approximately $1.2 \mathrm{~m}$ long and was pounded approximately two-thirds of its total length into the ground. One apex represented a reference point for repeat photography (not included in this report), and the heights of the other two rebar stakes were measured during each visit to assess deflation or aggradation of the ground surface. Crust compressive strength was measured vertically using a spring-piston-style pocket penetrometer. Surface shearstress measurements were made in situ with a hand-held shearvane tester, and sediment compaction was measured using a spear penetrometer. These measurements were made to evaluate changes in the playa surface and subsurface at these sites and were not intended for comprehensive description of playa sediment strength.

The second set of sites was established during the later part of the studies, and these sites were visited during a 29-month period from December 2006 through April 2009 (tables 4F, G, $H, I$, and $J$ ). A $10-\mathrm{m}^{2}$ plot was set up at each repeat site with four rebar stakes, one at each corner, and all repeat measurements were taken within the plot area. The location of each repeat measurement was noted so that these areas were avoided during future measurements. The rebar stakes were approximately $1.2 \mathrm{~m}$ long and were pounded into the ground during the initial site set-up so that the top of the rebar was approximately $45 \mathrm{~cm}$ above ground surface. These rebar heights were measured during each visit to assess deflation or aggradation of the ground surface. Small holes were dug in two places in the plot area per visit to determine the depth to moist or wet sediment. Differences in the measured depth to moisture between the two holes per visit are likely due to spatial variability within the plot area. During some sampling events, moist sediment was not intersected within the depth of excavation at the west and east transect sites. When this was the case, the measurement was designated as greater than the depth of the excavation (for example, $>70 \mathrm{~cm}$ ), or as "n.r." if the depth of the excavation was not recorded. Surface crust conductivities were measured with a portable conductivity meter, crust thickness (in millimeters) was measured using a caliper, and crust compressive strength was measured using a spring-piston-style pocket penetrometer. Samples were collected for mineralogical identification in the laboratory, and observations describing the ground-surface characteristics were recorded. 


\section{Groundwater Levels, Well Data, and Field Parameters}

Groundwater levels were measured from wells installed by Czarnecki (1997; "GS" and "Cz" wells). Measurements were also made from two new wells drilled in 2008 by the U.S. Geological Survey ("USGS" wells), as well as two previously installed but plugged wells that were re-drilled during USGS activities in 2008 ("Metal" wells). GS and Cz wells were measured 11-15 times over a 30-month period, and USGS and Metal wells were measured 2-5 times over a 12-month period to document groundwater-level fluctuations (table 5). Measurements were made with a standard weighted steel tape. All water levels were measured in feet and later converted to meters. Well depths were also measured at all well sites during the first visit to each site (table 5). Ground-surface elevations around most wells were measured using a GPS receiver and post-correcting, yielding an average vertical error of $20 \mathrm{~cm}$ (table 5).

During collection of groundwater samples, characteristics of the water that were measured in the field with standardized meters include $\mathrm{pH}$, temperature, and specific conductance (table 6). Standard titrations to determine the acid-neutralizing capacity were made at the sample site, or within 24 hours on an aliquot of the water sample that was stored with ice.

\section{Laboratory Analysis}

\section{Gravimetric Water Content}

Gravimetric water content was determined on select auger samples (table 7). During sampling, 15- to 20-g aliquots were stored immediately after excavation in pre-weighed glass scintillation vials fitted with polycone lids. The lids were tightened and wrapped with electrical tape to assure retention of moisture. The mass of sediment in each vial was measured upon return from the field and following heating for four days at $105^{\circ} \mathrm{C}$. The decrease in mass following heating is attributed to water loss including free water, nonstructural water in expansive clays, and structural water in some salt minerals.

Following determination of the water loss, the scintillation samples were placed in a chamber at 100 percent humidity to determine the water absorption capacity of the sediment. Samples were weighed weekly until the change in mass was less than 0.5 percent. This typically took six to ten weeks.

\section{Carbonate Percent}

Carbonate percent (table 8) was measured on a limited number of select grab samples using a Chittick apparatus (Dreimanis, 1962) as described by Machette (1986) whereby $6 \mathrm{~N} \mathrm{HCl}$ is applied to the sample and the gas evolved from the reaction displaces fluid within the Chittick apparatus. The volume of liquid displaced is used to calculate the percent of carbonate in the sample.

$$
\mathrm{CO}_{3} \%=\left(\mathrm{V}_{\mathrm{CO} 2} \times \mathrm{C}\right) / \mathrm{W}_{\mathrm{S}} \text {, }
$$

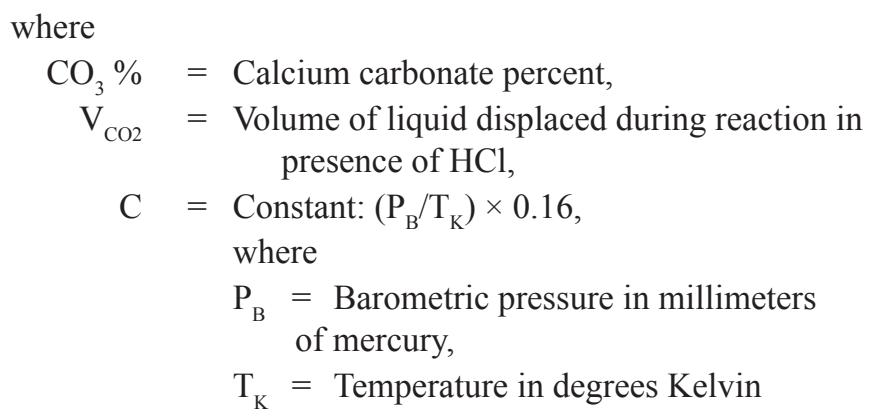

and

$\mathrm{W}_{\mathrm{S}}=$ Oven-dry sample weight (at $105^{\circ} \mathrm{C}$ for $16 \mathrm{hrs}$ ).

\section{Particle-Size Analysis}

Particle-size analysis was performed on the $<2$-mm-size fraction of select grab samples and east transect and coppicedune auger samples using a laser diffraction system. Prior to measurement of particle size, the samples were digested to remove organic matter and carbonate minerals using 30 percent $\mathrm{H}_{2} \mathrm{O}_{2}$ and 10 percent $\mathrm{HCl}$, respectively. Sodium hexametaphosphate was added to each sample to aid in the suspension of clay particles. Measurements were made using a Malvern laser analyzer. The samples were introduced into an aqueous medium and pumped through the laser analyzer for particle-size measurements. Thus, the particle size reported here represents the nonwater-soluble fraction. Particle size is reported in accordance with the Wentworth (Wentworth, 1922) scale (tables 9A, $B$, and $C$ ).

\section{Bulk Density}

Sediment bulk density (table 10) was determined by using either a 1- or 3-cm-tall brass ring, each having a diameter of $5.4 \mathrm{~cm}$ and thus a known volume. The rings were pressed into the sediment, excavated, and excess sediment was shaved flush to the ring with a trowel. The sample was then dried and weighed. The bulk density is expressed in grams of sediment per cubic centimeter $\left(\mathrm{g} / \mathrm{cm}^{3}\right)$.

\section{Geochemical Analysis}

\section{Solid Samples}

Samples were analyzed to determine their bulk chemical composition using inductively coupled plasma-optical emission spectrometry (ICP-OES; Briggs, 2002a) and inductively coupled plasma-mass spectrometry (ICP-MS; Briggs and Meier, 2002) following acid digestion. Samples collected by hand auger were sieved to obtain the $<2$-mm-size fraction that was analyzed (table 11A). The $<2$-mm- and $<53$-mm-size fractions (tables $11 B$ and $C$ ) of grab samples were also analyzed using ICP-OES and ICP-MS. All sediment samples were finely ground using a shatterbox prior to analysis.

\section{Leach Samples}

Deionized (DI) water and 1-percent nitric acid leachates were prepared to determine the water soluble, acid soluble, and final residual components of selected sediment samples 
(table 12). Chemical analysis was completed on leachates and final residuals from the east transect auger hole bulk samples (tables $12 A, B$, and $C$ ), and on leachates and final residuals from selected bulk (tables $12 D, E$, and $F$ ) and fine-fraction (tables $12 G, H$, and $I$ ) grab samples. The steps of leaching were DI water $\rightarrow$ nitric acid $\rightarrow$ final residual. Thirty milliliters of DI water was added to approximately $1 \mathrm{~g}$ of solid sample and shaken for 1 hour. After shaking, the samples were centrifuged to remove suspended aluminosilicate particles that were greater than $0.05 \mu \mathrm{m}$ in diameter. The leachate was then passed through a $0.2-\mathrm{mm}$ filter to remove organic particles and solids that were resuspended during transfer of the leachate. Measurements of $\mathrm{pH}$ were made using $\mathrm{pH}$ indicator strips or using a $\mathrm{pH}$ meter on a separated aliquot. The resulting leachate was split into two subsamples for chemical analysis: one split for anion analysis (IC-ion chromatograph; Theodorakos and others, 2002) and the second split for cation analysis (ICP-MS; Lamothe and others, 2002; ICP-OES; Briggs, 2002b). The split for cation analysis was stabilized by adding concentrated nitric acid until a $\mathrm{pH}<2$ was obtained. The anion split was refrigerated. Separate splits for alkalinity determinations (tables $12 A, D$, and $G$ ) were prepared as a DI-water leach and were not stabilized. Initial $\mathrm{pH}$ measurements are reported in tables $12 A, D$, and $G$ and include those that were made during alkalinity determinations or those made during DI leaching using $\mathrm{pH}$ strips. The solid residue remaining after the DI-water leach was dried and weighed, then combined with 1-percent nitric acid at a 1:30 weight:volume ratio. The samples were shaken for 1 hour, then centrifuged and filtered using the same protocol as the DI-water leaching. Measurements of $\mathrm{pH}$ were made on the resulting leachate, and if necessary, the leachate was stabilized to a $\mathrm{pH}<2$ using concentrated nitric acid. The leachate was submitted for chemical analysis by ICP-MS and ICP-OES methods. The final residue remaining after the nitric leach was dried, weighed, ground, and submitted for total chemical analysis by ICP-MS and ICP-OES methods. Some final residues were very low in weight so that only one analytical method (ICP-MS) was possible.

\section{Water Samples}

A summary of field and laboratory water analyses is listed in table 3. Water samples were analyzed (table 13) for the concentration of several major, minor, and trace elements, and anions. In addition, the hydrogen $(\delta \mathrm{D})$ and oxygen $\left(\delta^{18} \mathrm{O}\right)$ isotopic composition of the water, and sulfur $\left(\delta^{34} \mathrm{~S}\right)$ and oxygen $\left({ }^{18} \mathrm{O}\right)$ in dissolved sulfate were measured. Major cation, minor, and trace elements were determined by ICP-OES (Briggs, 2002b) and ICP-MS (Lamothe and others, 2002). Major anion concentrations were determined by ion chromatography (IC; Theodorakos and others, 2002 ). Analyses of replicate water samples for the ICP and IC analyses indicated a precision of 5 to 10 relative percent for all measured constituents. Determinations of $\delta \mathrm{D}$ and $\delta^{18} \mathrm{O}$ in water were made at the University of Arizona Environmental Isotope Laboratory. Chromium reduction was used to determine $\delta \mathrm{D}$, and it was measured using isotope-ratio mass spectrometry (Gehre and others, 1996). Determination of $\delta^{18} \mathrm{O}$ was through equilibrium with $\mathrm{CO}_{2}$ measured using isotoperatio mass spectrometry (Craig, 1957).

Dissolved sulfate was precipitated as $\mathrm{BaSO}_{4}$ from filtered, weakly acidified ( $\mathrm{pH} 3-4)$ water samples, and the $\mathrm{BaSO}_{4}$ was analyzed for sulfur $\left(\delta^{34} \mathrm{~S}\right)$ and oxygen $\left(\delta^{18} \mathrm{O}\right)$ isotopes using continuous-flow isotope-ratio analysis coupled with a thermal combustion/elemental analyzer system. Analyses were completed at the USGS Stable Isotope Laboratory, Denver, Colo. Sulfur values were normalized using IAEA-SO-6 and NBS 127 or sulfide standards IAEA-S-3 and NBS 123 to Vienna Cañon Diablo Troilite (VCDT). Replicate analyses reproduced to within $\pm 0.1 \%$ (one-sigma). Oxygen values were normalized to in-house barium sulfate standards (Wasserman and others, 1992). Replicate analyses reproduced to within $\pm 0.3 \%$.

\section{Electrical Conductivity}

Electrical conductivity was measured in the field on repeatsite crust samples (tables $4 F, G, H$, $I$, and $J$ ) and in the laboratory on bulk auger-hole samples and a few bulk grab samples (tables $14 A$ and $B$ ). Field measurements were made using an electrical conductivity meter on a sediment-water mixture. One level teaspoon of sediment was combined with $250 \mathrm{~mL}$ of de-ionized water. The mixture was immediately shaken to suspend the solid and shaken again after 20 minutes. Following a 10-minute settling time the electrical conductivity of the suspension was measured. Lab measurements were made on a 30:1 DI-water-to-sediment mixture as in the leaching methods. After the mixture was shaken and centrifuged, the electrical conductivity was measured.

\section{X-Ray Diffraction}

The mineral composition of the sampled sediments was determined using powder X-ray diffraction techniques. Several fractions of the samples were characterized (table 15) including bulk solid, DI residue (bulk and fine fractions), final residue after nitric acid leaching (bulk and fine fractions), bulk clay, and bulk solid as a slurry. All data were collected using copper $\mathrm{K} \alpha$ radiation using a Philips XRG 3100 and a Norelco goniometer equipped with a graphite monochromater. Phase identification was facilitated using JADE software by Materials Data, Inc.

\section{Acknowledgments}

We thank the manager of the Ash Meadows National Wildlife Refuge for graciously granting permission to sample water and surficial materials within the Refuge; Rian Bogle, Miguel Velasco, and John Vogel for field assistance; Jiang Xiao and Todd Preston for sediment preparation and analyses; Darren "Paco" Van Sistine for GIS work; and Jeremy Havens for graphics advice. Thanks also to Kenzie Turner and Jeff Honke for review of this report and helpful suggestions for improvement. 


\section{References Cited}

Breit, G., Goldstein, H., Reynolds, R.L., and Yount, J.C., 2009, Distribution of major anions and trace elements in the unsaturated zone beneath Franklin Lake playa, California, in Oren, A., Naftz, D.L., and Wurtsbaugh, W.A., eds., Saline lakes around the world: Unique systems with unique values: Natural Resources and Environmental Issues, v. 15, p. 93-103 (ISSN 1069-5370).

Briggs, P.H., 2002a, The determination of forty elements in geological and botanical samples by inductively coupled plasma-atomic emission spectrometry, chap. G of Taggart, J.E., ed., Analytical methods for chemical analysis of geologic and other materials: U.S. Geological Survey OpenFile Report 02-223, 20 p.

Briggs, P.H., 2002b, The determination of twenty-seven elements in aqueous samples by inductively coupled plasma-atomic emission spectrometry, chap. F of Taggart, J.E., ed., Analytical methods for chemical analysis of geologic and other materials: U.S. Geological Survey Open-File Report 02-223, 13 p.

Briggs, P.H., and Meier, A.L., 2002, The determination of fortytwo elements in geological materials by inductively coupled plasma-mass spectrometry, chap. I of , Taggart, J.E., ed., Analytical methods for chemical analysis of geologic and other materials: U.S. Geological Survey Open-File Report 02-223, $16 \mathrm{p}$.

Craig, H., 1957, Isotopic standards for carbon and oxygen and correction factors for mass spectrometric analysis of carbon dioxide: Geochimica et Cosmochimica Acta, v. 12, p. 133-149.

Czarnecki, J.B., 1997, Geohydrology and evapotranspiration at Franklin Lake playa, Inyo County, California: U.S. Geological Survey Water-Supply Paper 2377, 75 p.

Dreimanis, A., 1962, Quantitative gasometric determination of calcite and dolomite by using Chittick apparatus: Journal of Sedimentary Petrology, v. 32, no. 3, p. 520-529.

Gehre, M., Heofling, R., Kowski, P., and Strauch, G., 1996, Sample preparation device for quantitative hydrogen isotope analysis using chromium metal: Analytical Chemistry, v. 68, p. 4414-4417.
Lamothe, P.J., Meier, A.L., and Wilson, S.A., 2002, The determination of forty four elements in aqueous sample by inductively coupled plasma-mass spectrometry, chap. H. of Taggart, J.E., ed., Analytical methods for chemical analysis of geologic and other materials: U.S. Geological Survey Open-File Report 02-223, 13 p.

Machette, M., 1986, Calcium and magnesium carbonates, in Singer, M., and Janitzky, P., eds., Field and laboratory procedures used in a soil chronosequence study: U.S. Geological Survey Bulletin 1648, p. 30-33.

Reheis, M.C., 1997, Dust deposition downwind of Owens (dry) Lake, 1991-1994: Preliminary findings: Journal of Geophysical Research, 102D, p. 25999-26008.

Reheis, M.C., and Kihl, R., 1995, Dust deposition in southern Nevada and California, 1984-1989: Relations to climate, source area, and lithology: Journal of Geophysical Research 100D5, p. 8893-8918.

Reynolds, R.L., Bogle, R., Vogel, J., Goldstein, H., and Yount, J.C., 2009, Dust emission at Franklin Lake playa, Mojave Desert (USA): Response to meteorological and hydrologic changes 2005-2008, in Oren, A., Naftz, D.L., and Wurtsbaugh, W.A., eds., Saline lakes around the world: Unique systems with unique values: Natural Resources and Environmental Issues v. 15, p. 105-116 (ISSN 1069-5370).

Reynolds, R.L., Yount, J.C., Reheis, M.C., Goldstein, H., Chavez, P., Jr., Fulton, R., Whitney J., Fuller, C., and Forester, R.M., 2007, Dust emission from wet and dry playas in the Mojave Desert: Earth Surface Processes and Landforms, v. 32, p. 1811-1827, DOI: 10.1002/esp.1515.

Theodorakos, P.M., d'Angelo, W.M., and Ficklin, W.H., 2002, Fluoride, chloride, nitrate, and sulfate in aqueous solution utilizing AutoSuppression chemically suppressed ion chromatography, chap. V of Taggart, J.E., ed., Analytical methods for chemical analysis of geologic and other materials: U.S. Geological Survey Open-File Report 02-223, 9 p.

Wasserman, M.D., Rye, R.O., Bethke, P.M., and Arribas, Jr., A.,1992, Methods for separation and total stable isotope analysis of alunite: U.S. Geological Survey Open-File Report 92-9, 20 p.

Wentworth, C.K., 1922, A scale of grade and class terms for clastic sediments: Journal of Geology, v. 30, p. 377-392. 


\section{Tables}


Table 1. Location information for sampling sites.

[Sample site, name of sampling site; Surface characteristic, general nature of the ground surface around the sample site; Collection method, method of sample collection; Sampling designation, sample labeling designation; WC, water chemistry sampling; WM, groundwater level measurement; *, date designates time of repeat site or dust trap site set-up]

\begin{tabular}{|c|c|c|c|c|c|c|c|}
\hline Sample site & $\begin{array}{c}\text { Surface } \\
\text { characteristic }\end{array}$ & $\begin{array}{c}\text { Collection } \\
\text { method }\end{array}$ & $\begin{array}{c}\text { Sampling } \\
\text { designation }\end{array}$ & Latitude & Longitude & Datum & $\begin{array}{c}\text { Collection } \\
\text { date }\end{array}$ \\
\hline East Transect Auger & Porous and soft & Auger & HG07-04 & 36.254 & -116.368 & WGS84 & $11 / 12 / 2007$ \\
\hline East Transect Auger & Porous and soft & Auger & FPAH02-09 & 36.254 & -116.368 & WGS84 & $2 / 25 / 2009$ \\
\hline East Transect Auger & Porous and soft & Auger & FPAH04-09 & 36.254 & -116.368 & WGS84 & $4 / 7 / 2009$ \\
\hline Around East Transect Auger & Porous and soft & Grab & HG07-05 & 36.254 & -116.368 & WGS84 & $11 / 12 / 2007$ \\
\hline Around East Transect Auger & Porous and soft & Grab & HG07-08 & 36.254 & -116.369 & WGS84 & $11 / 12 / 2007$ \\
\hline Around East Transect Auger & Porous and soft & Grab & HG07-09 & 36.254 & -116.369 & WGS84 & $11 / 12 / 2007$ \\
\hline Clay Dune Auger & Porous and soft & Auger & HG09-01 & 36.267 & -116.357 & WGS84 & $2 / 23 / 2009$ \\
\hline Discovery Auger & Porous and soft & Auger & HG09-02 & 36.230 & -116.381 & WGS84 & $2 / 24 / 2009$ \\
\hline Discovery Auger & Porous and soft & Auger & DSAH04-07 & 36.230 & -116.381 & WGS84 & $4 / 11 / 2007$ \\
\hline Discovery Auger & Porous and soft & Auger & DSAH04-07b & 36.230 & -116.381 & WGS84 & $4 / 11 / 2007$ \\
\hline Ash Meadows & $\begin{array}{l}\text { Spring discharge/ } \\
\text { porous and soft }\end{array}$ & Grab & JY03-09 & 36.425 & -116.364 & WGS84 & $1 / 27 / 2003$ \\
\hline Obelisk & $\begin{array}{l}\text { Vegetated and } \\
\text { desert pavement }\end{array}$ & Grab & JY03-22 & 36.287 & -116.375 & WGS84 & $5 / 11 / 2003$ \\
\hline Ojo del Caballo & $\begin{array}{c}\text { Braided stream channel/ } \\
\text { porous and soft }\end{array}$ & Grab & JY03-23 & 36.285 & -116.369 & WGS84 & $5 / 11 / 2003$ \\
\hline Ojo del Caballo & $\begin{array}{c}\text { Braided stream channel/ } \\
\text { porous and soft }\end{array}$ & Grab & JY03-24 & 36.285 & -116.368 & WGS84 & $5 / 11 / 2003$ \\
\hline Salt Pan & Salt pan & Grab & JY03-25 & 36.268 & -116.354 & WGS84 & $5 / 11 / 2003$ \\
\hline Salt Pan & Salt pan & Grab & JY03-26 & 36.267 & -116.354 & WGS84 & $5 / 11 / 2003$ \\
\hline Salt Pan & Salt pan & Grab & JY03-27 & 36.267 & -116.353 & WGS84 & $5 / 11 / 2003$ \\
\hline East of East Transect & Hard and compact & Grab & JY03-28 & 36.253 & -116.350 & WGS84 & $5 / 11 / 2003$ \\
\hline East Transect & Hard and compact & Grab & JY03-29 & 36.253 & -116.355 & WGS84 & $5 / 11 / 2003$ \\
\hline Ash Meadows & $\begin{array}{l}\text { Spring discharge/ } \\
\text { porous and soft }\end{array}$ & Grab & JY03-50 & 36.428 & -116.362 & WGS84 & $8 / 19 / 2003$ \\
\hline Franklin Lake Playa/Carson Slough & $\begin{array}{l}\text { Braided stream channel/ } \\
\text { porous and soft }\end{array}$ & Grab & JY04-01 & 36.323 & -116.365 & WGS84 & $1 / 31 / 2004$ \\
\hline Coppice & Coppice dunes & Grab & JY04-15 & 36.239 & -116.384 & WGS84 & $2 / 4 / 2004$ \\
\hline Coppice & Coppice dunes & Grab & JY04-16 & 36.239 & -116.384 & WGS84 & $2 / 4 / 2004$ \\
\hline North of Clay Dune & Salt pan & Grab & JY04-17 & 36.269 & -116.355 & WGS84 & $2 / 4 / 2004$ \\
\hline Discovery & Porous and soft & Grab & JY05-12 & 36.230 & -116.381 & WGS84 & $4 / 15 / 2005$ \\
\hline Discovery & Porous and soft & Grab & JY05-13 & 36.230 & -116.381 & WGS84 & $4 / 15 / 2005$ \\
\hline Coppice & Coppice dunes & Grab & JY05-14 & 36.239 & -116.384 & WGS84 & $4 / 15 / 2005$ \\
\hline Discovery & Porous and soft & Grab & JY-05-15 & 36.230 & -116.381 & WGS84 & $4 / 15 / 2005$ \\
\hline Discovery & Porous and soft & Grab & JY-05-16 & 36.230 & -116.381 & WGS84 & $4 / 15 / 2005$ \\
\hline Coppice & Coppice dunes & Grab & JY-05-17 & 36.239 & -116.384 & WGS84 & $4 / 15 / 2005$ \\
\hline West Transect & Braided stream channel & Grab & JY05-18 & 36.254 & -116.380 & WGS84 & $4 / 16 / 2005$ \\
\hline West Transect & Braided stream channel & Grab & JY-05-19 & 36.254 & -116.380 & WGS84 & $4 / 16 / 2005$ \\
\hline East Transect & Hard and compact & Grab & JY05-20 & 36.253 & -116.355 & WGS84 & $4 / 16 / 2005$ \\
\hline East Transect & Hard and compact & Grab & JY-05-21 & 36.253 & -116.355 & WGS84 & $4 / 16 / 2005$ \\
\hline Salt Pan & Salt pan & Grab & JY05-22 & 36.267 & -116.355 & WGS84 & $4 / 16 / 2005$ \\
\hline Salt Pan & Salt pan & Grab & JY-05-23 & 36.267 & -116.355 & WGS84 & $4 / 16 / 2005$ \\
\hline
\end{tabular}


Table 1. Location information for sampling sites.-Continued

[Sample site, name of sampling site; Surface characteristic, general nature of the ground surface around the sample site; Collection method, method of sample collection; Sampling designation, sample labeling designation; WC, water chemistry sampling; WM, groundwater level measurement; *, date designates time of repeat site or dust trap site set-up]

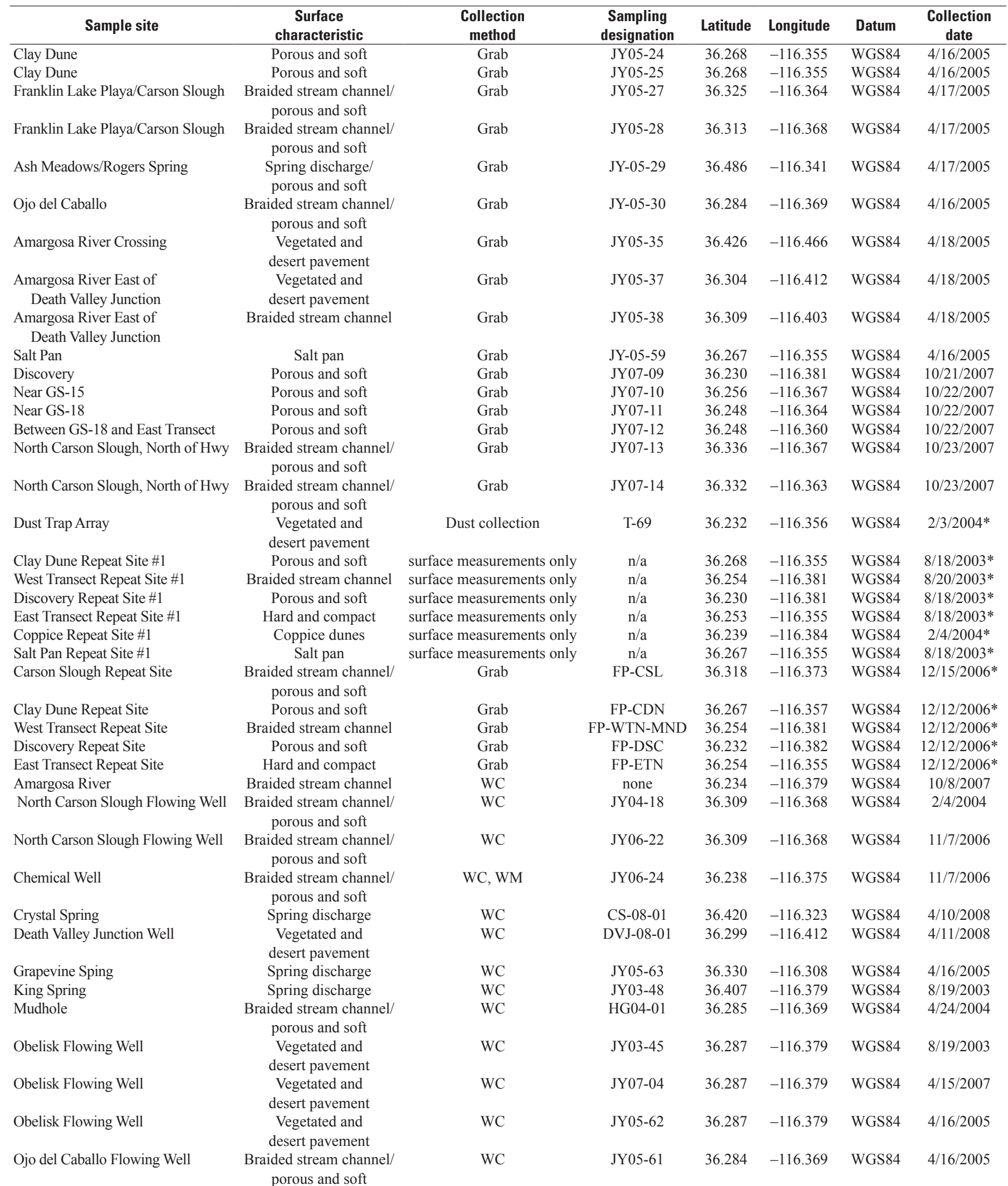


Table 1. Location information for sampling sites.-Continued

[Sample site, name of sampling site; Surface characteristic, general nature of the ground surface around the sample site; Collection method, method of sample collection; Sampling designation, sample labeling designation; WC, water chemistry sampling; WM, groundwater level measurement; *, date designates time of repeat site or dust trap site set-up]

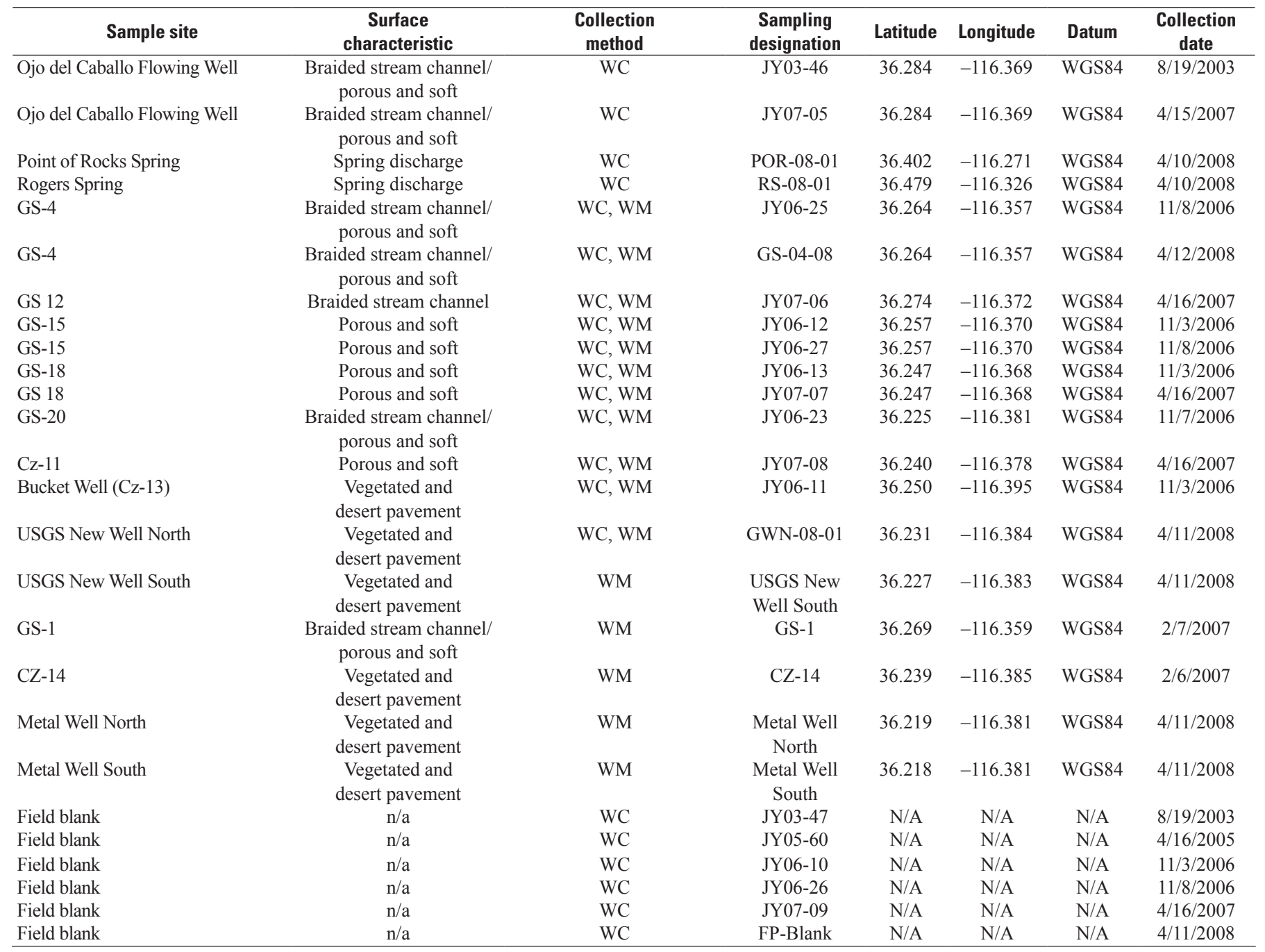


Tables 17

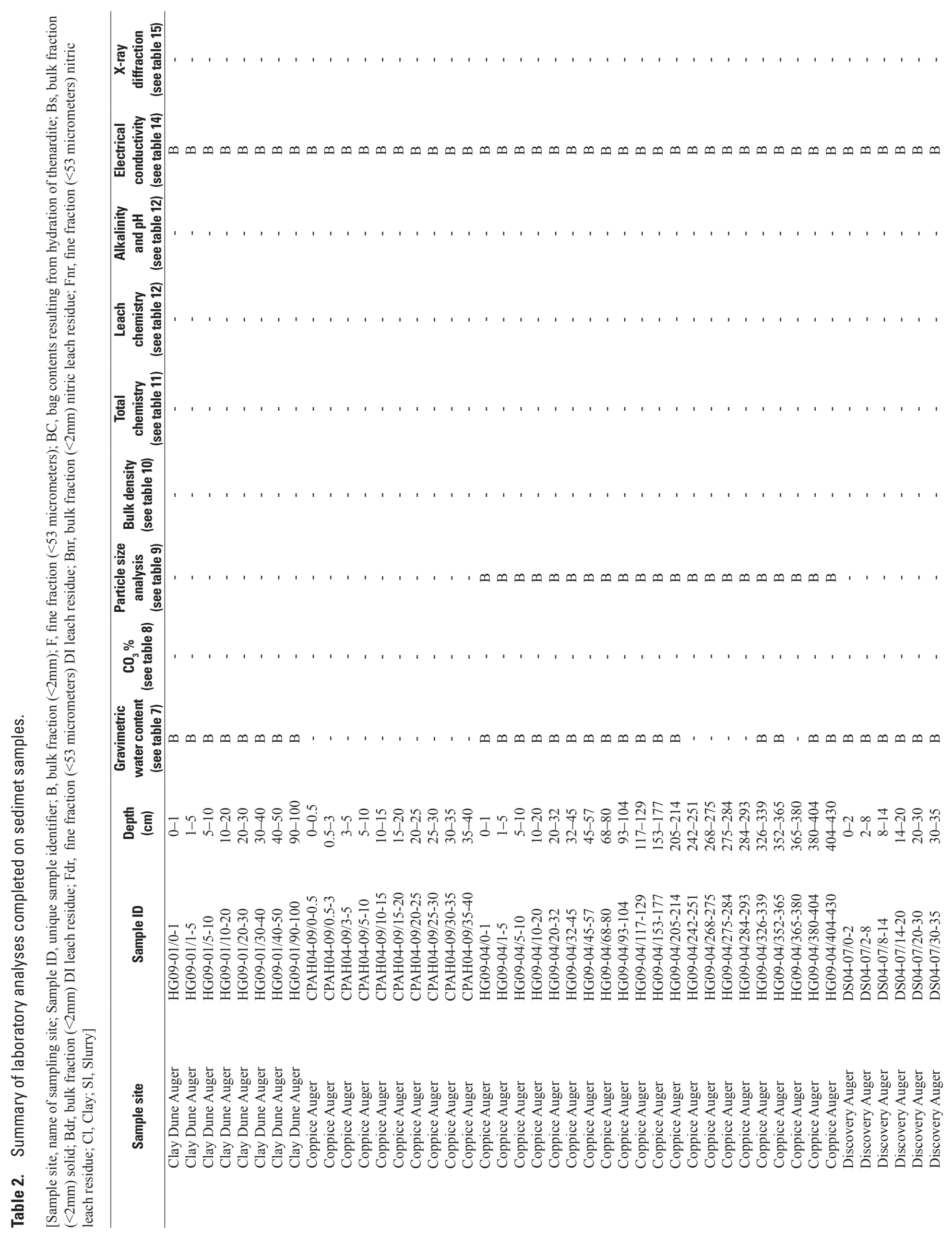




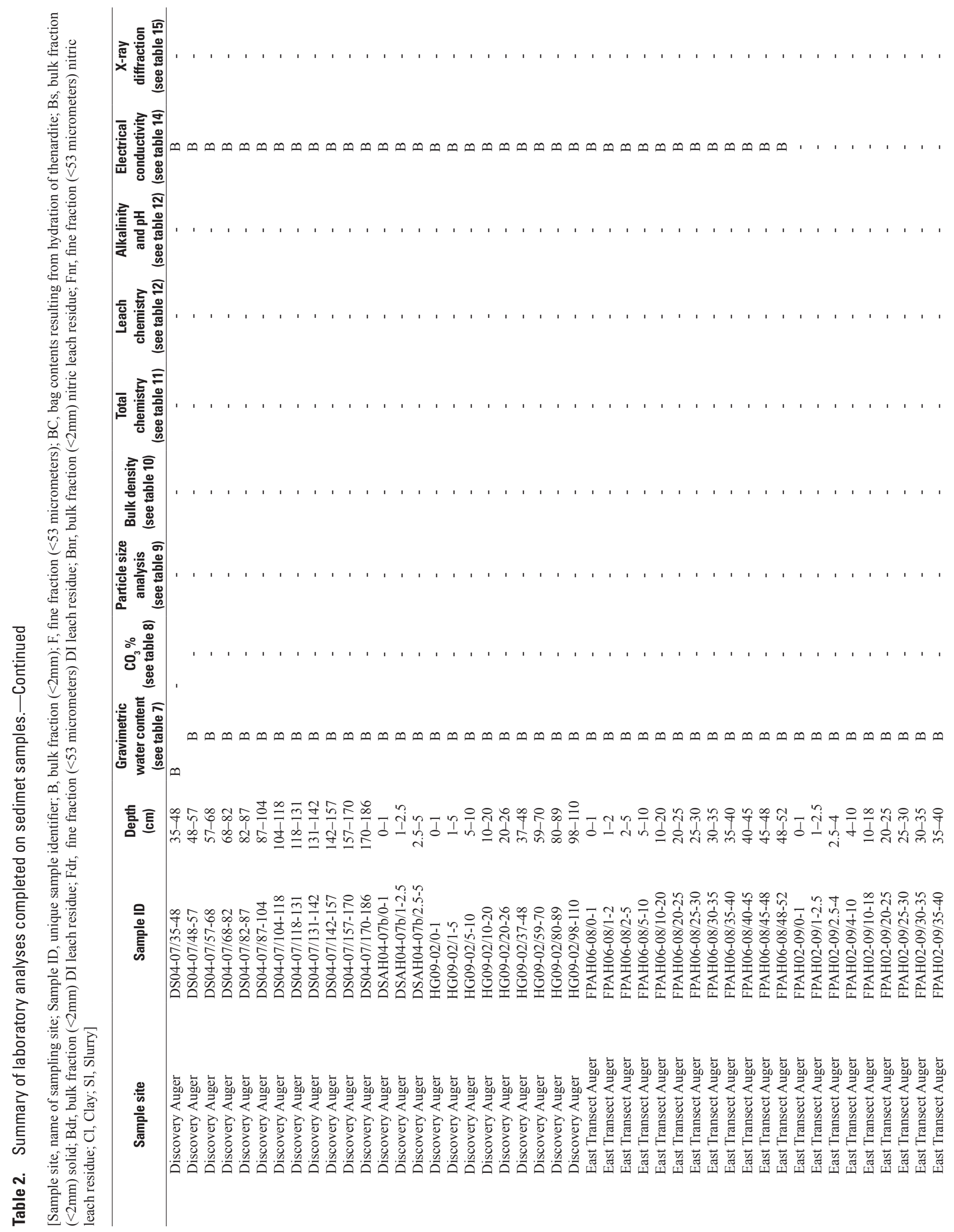




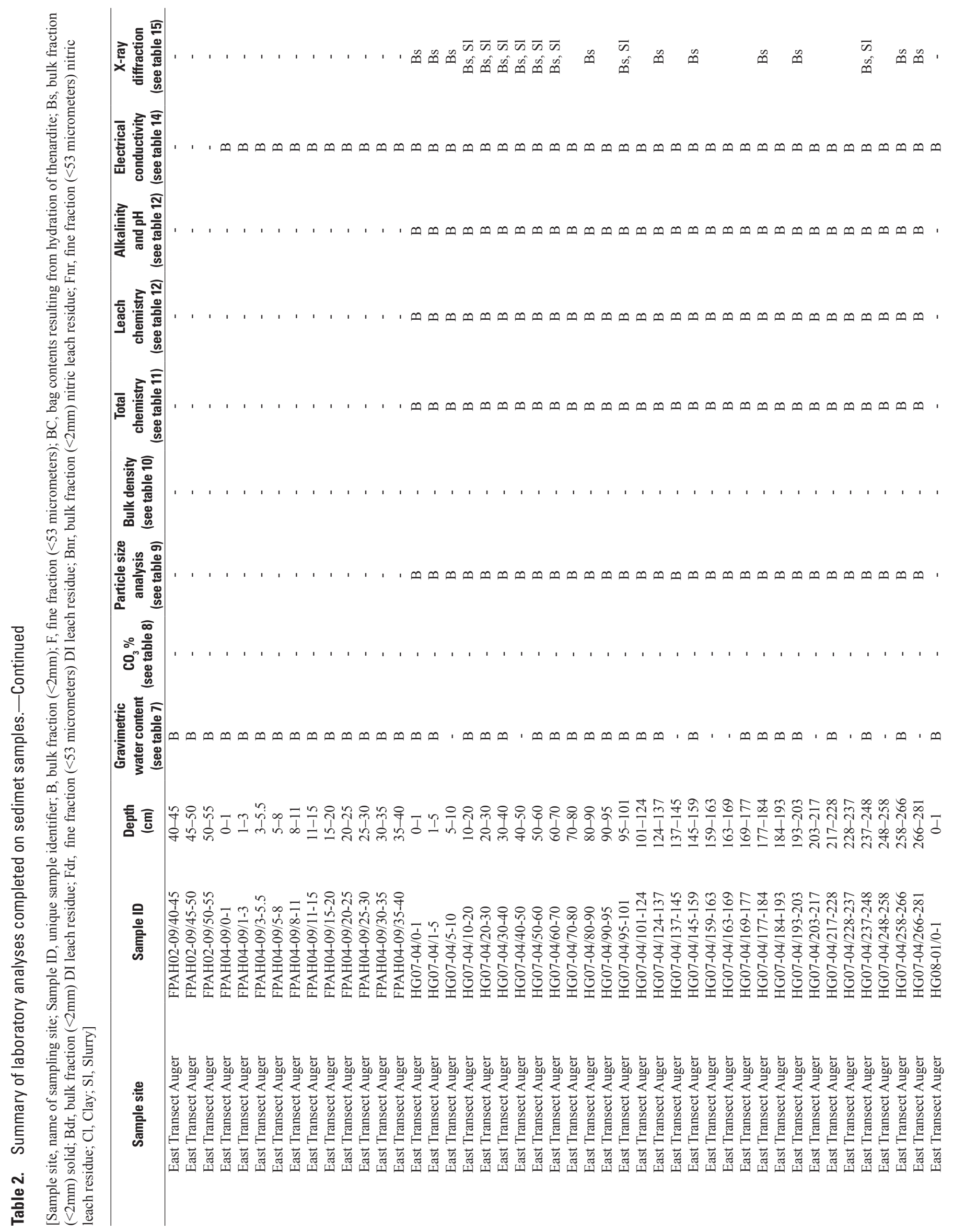




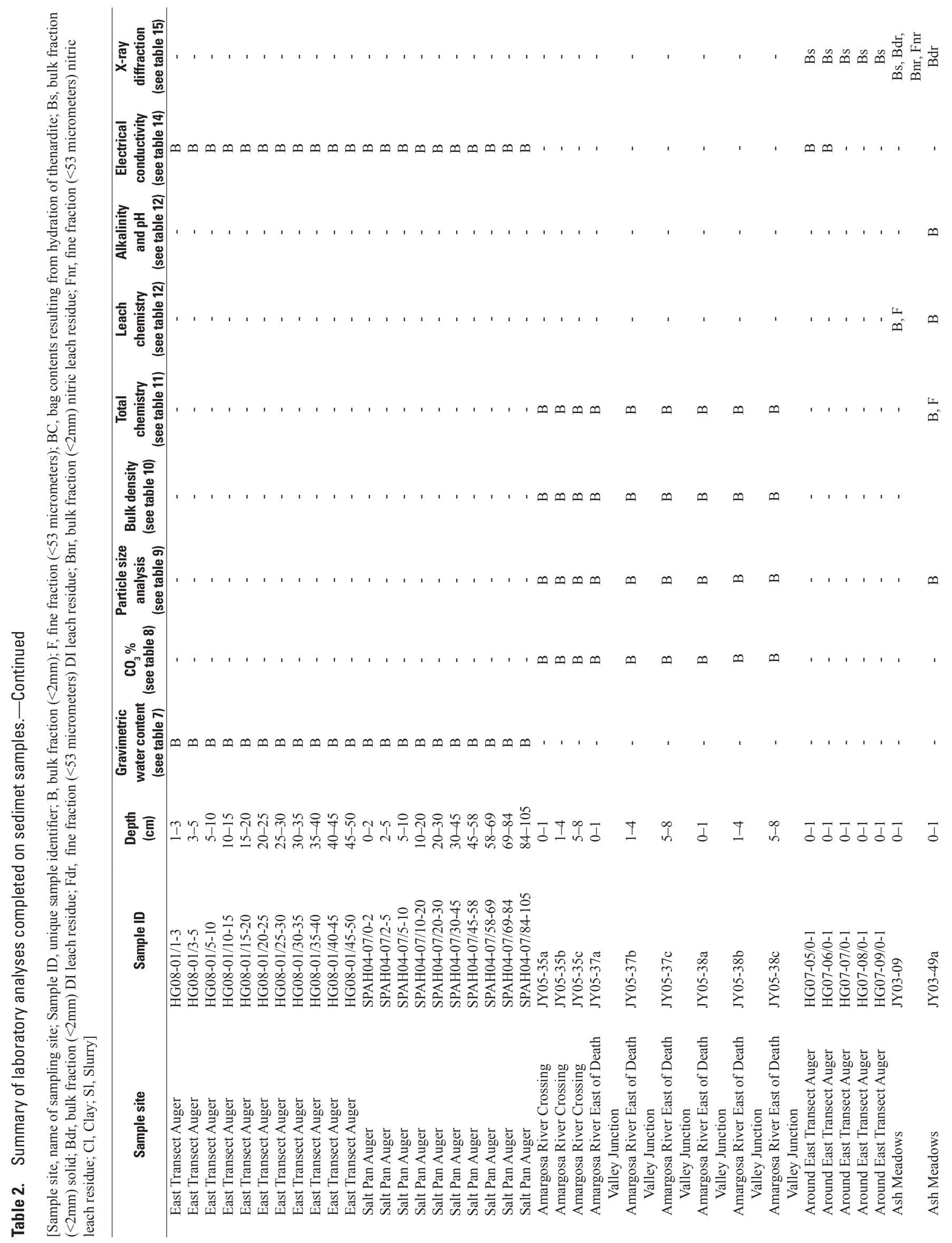




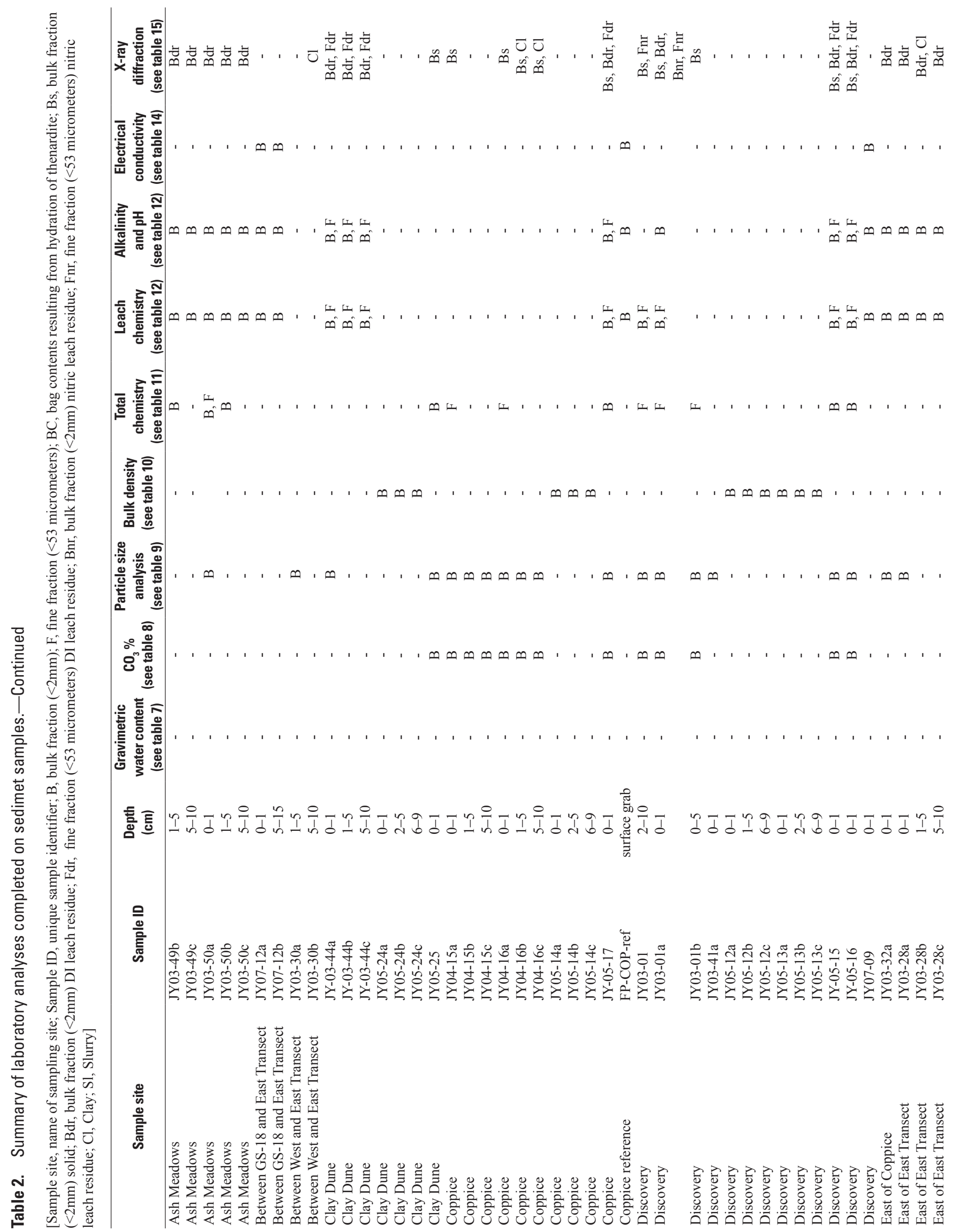




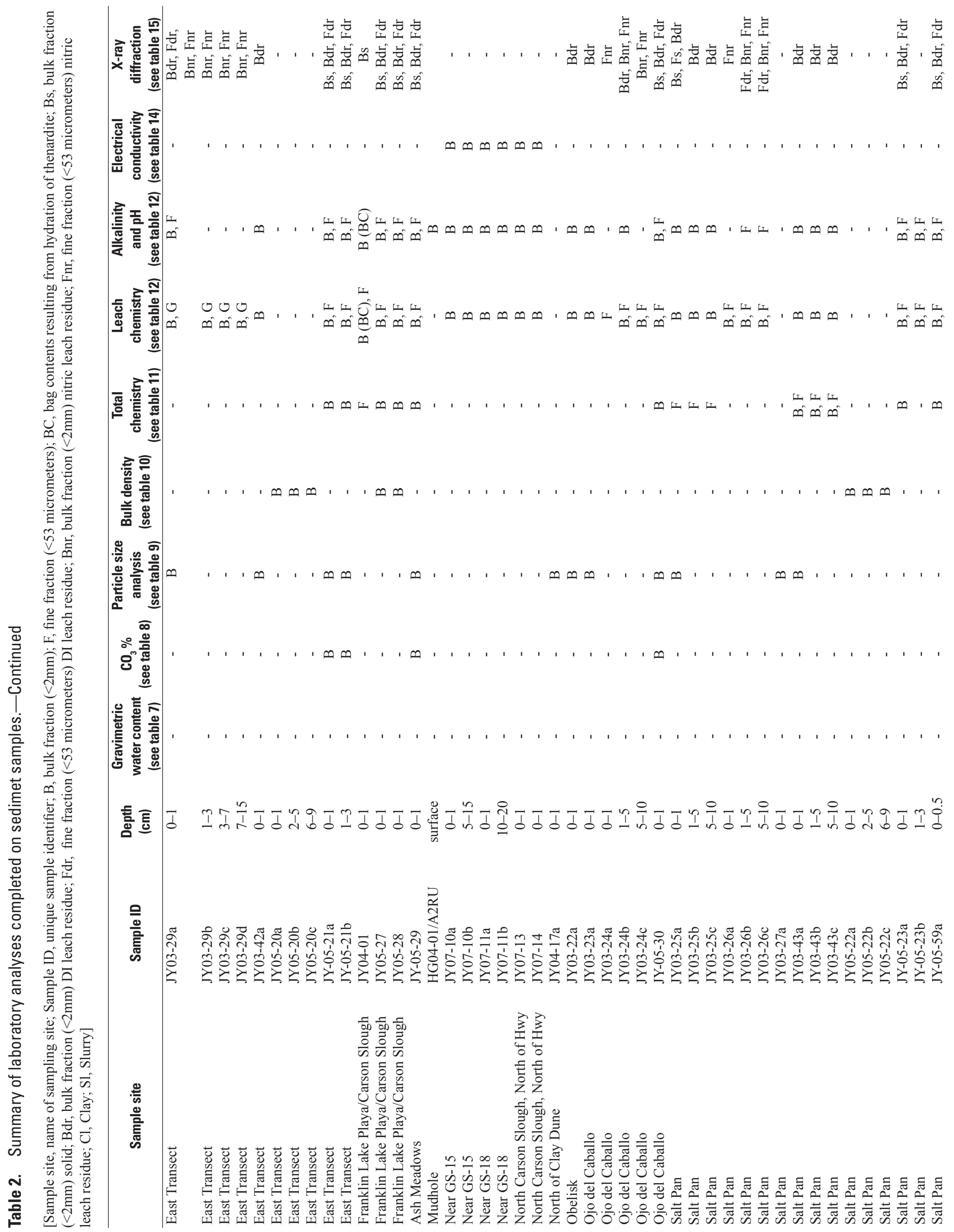




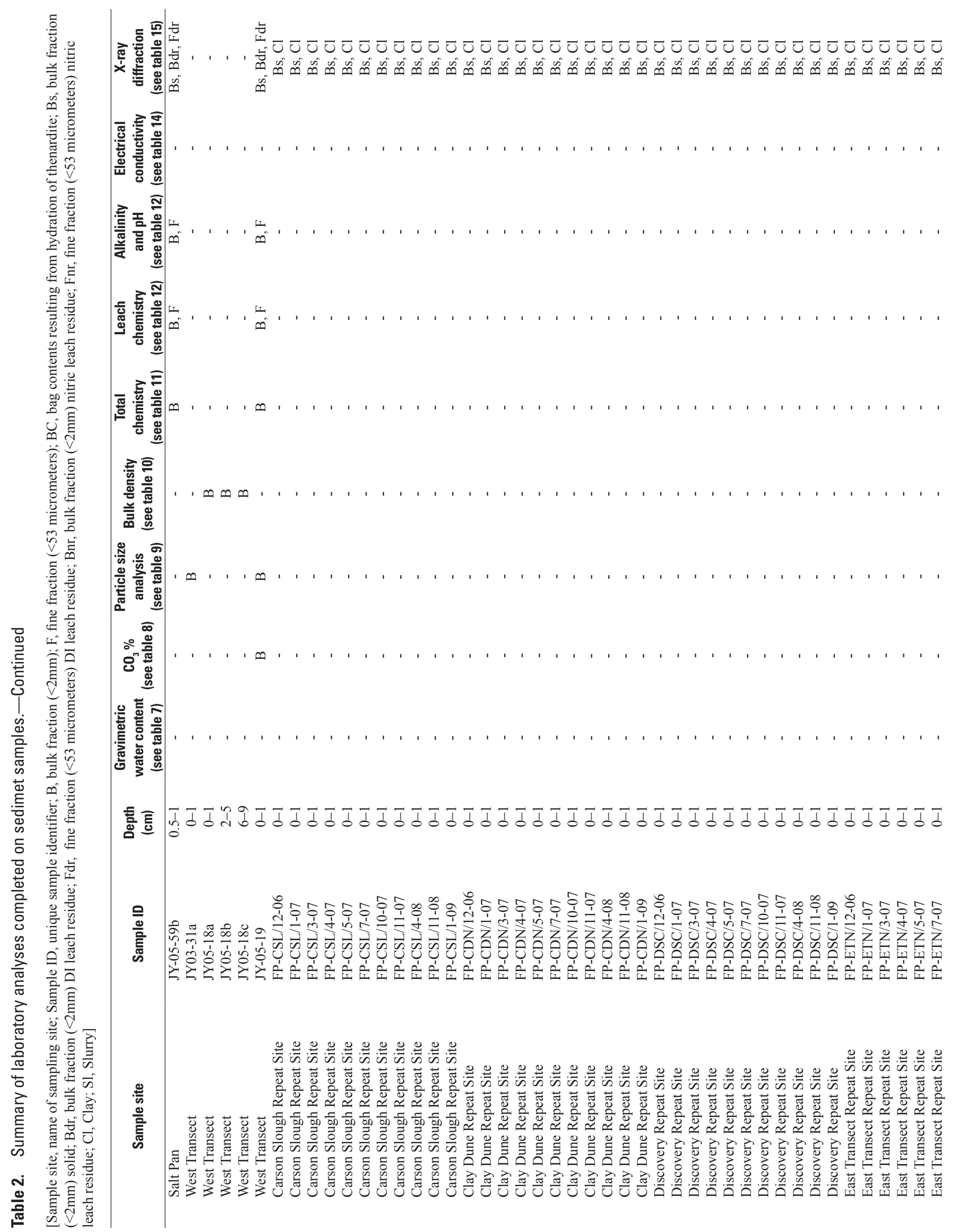




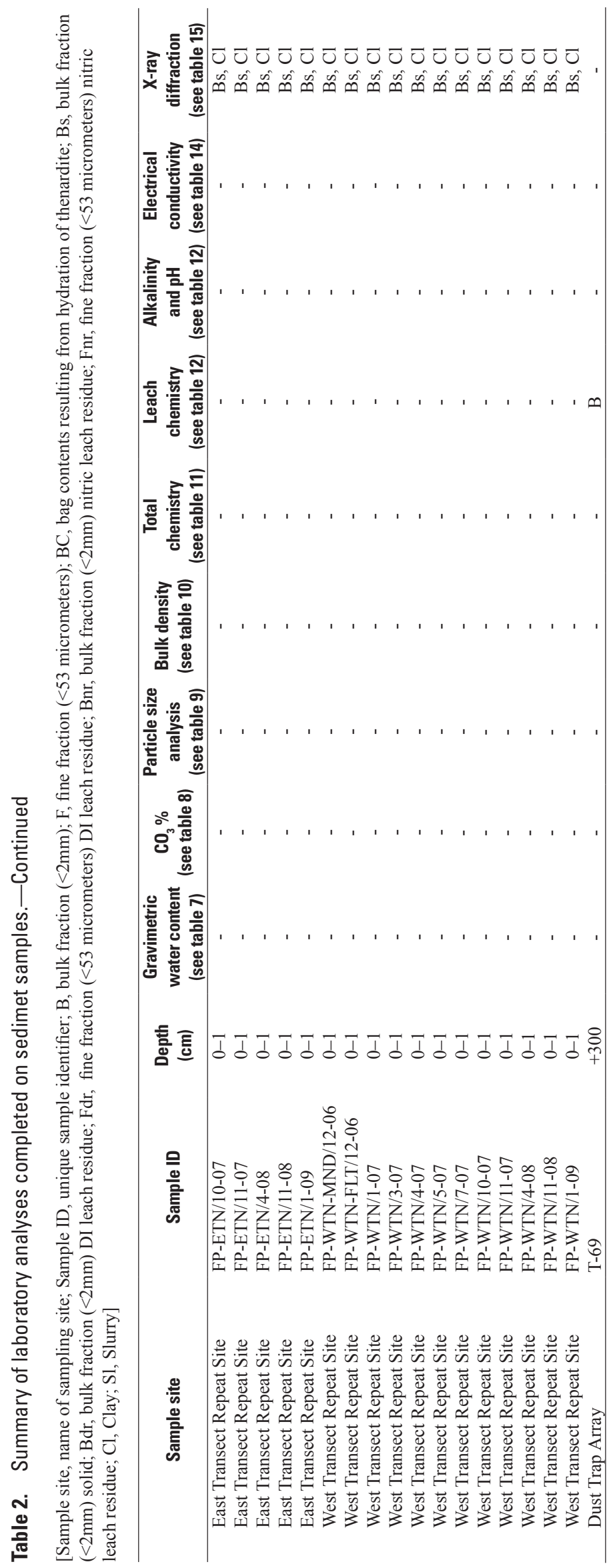




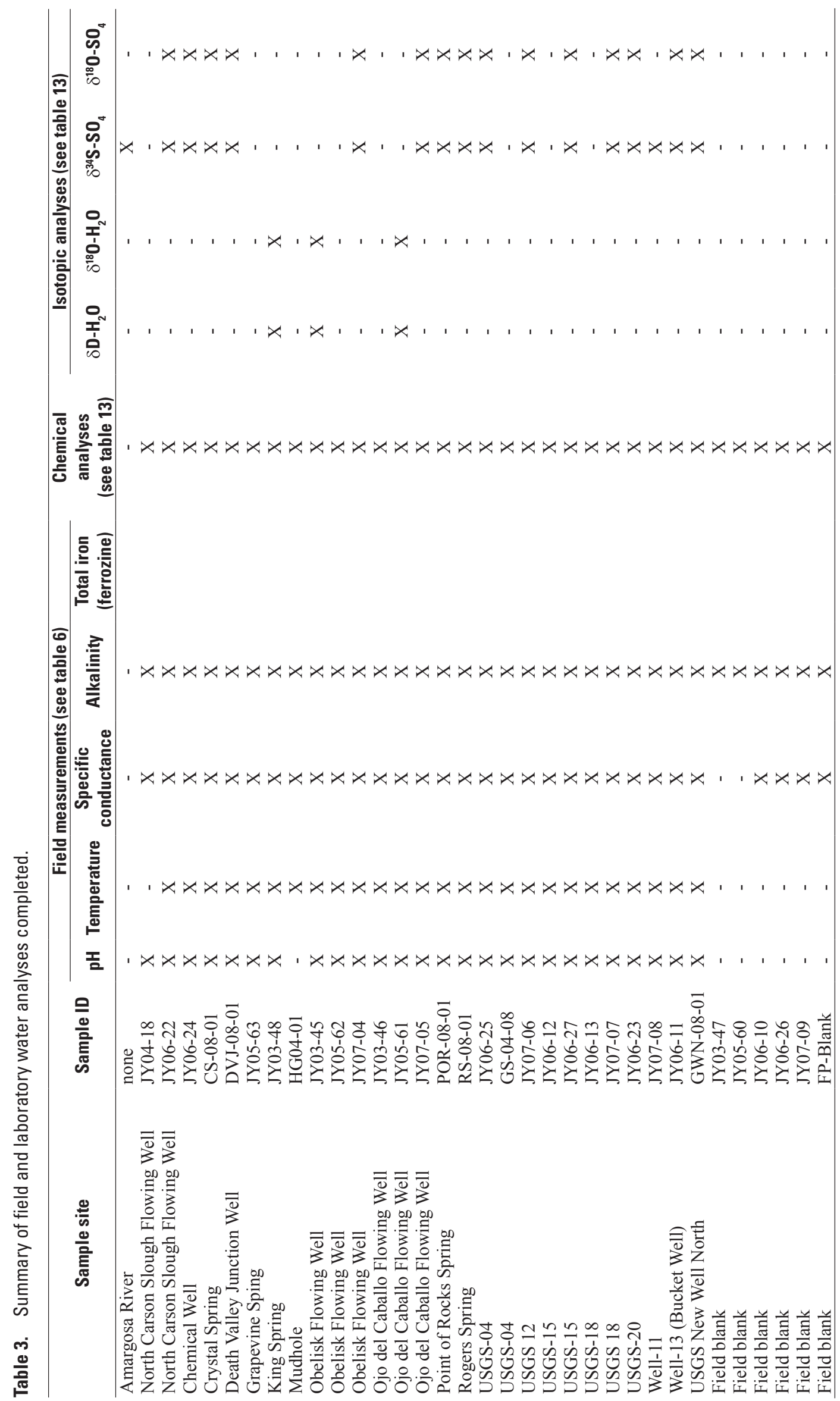


Table 4A. Repeat measurement data for Coppice Dune repeat site \#1.

[n.d., not determined; Date, date measurements were made; Pin 1, 2, height of top of erosion pin above the ground surface; Compressive strength, vertical compressive strength of ground surface measured with a spring-piston-style pocket penetrometer; Shear stress, horizontal shear stress of ground surface measured with a hand-held shear vane; Sub-surface compaction, vertical sub-surface compaction measured in pounds per square inch (PSI) using a spear penetrometer; $\mathrm{cm}, \mathrm{centimeters;} \mathrm{kg} / \mathrm{cm}^{2}$, kilograms per centimeter squared; “, inches]

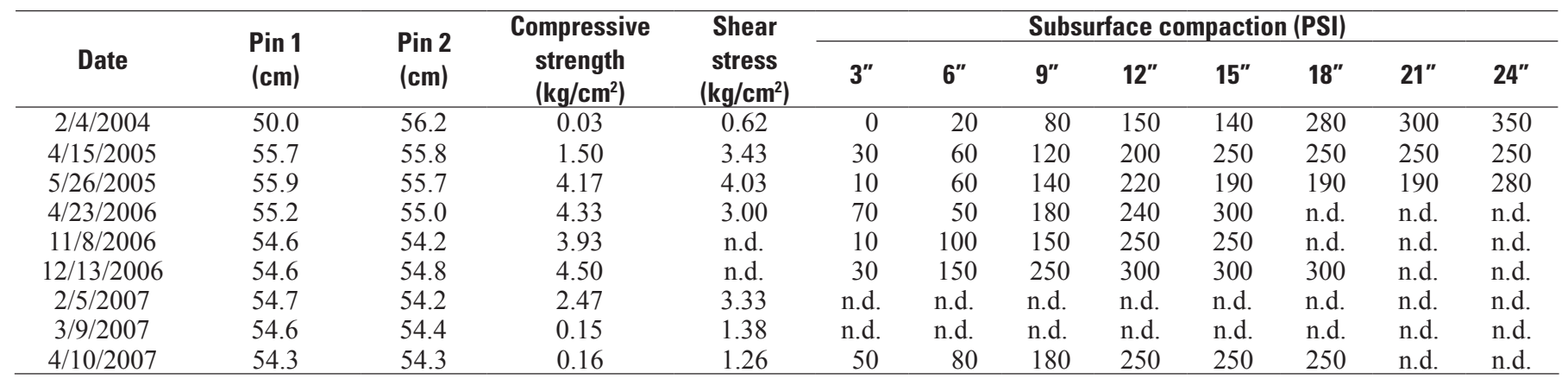

Table 4B. Repeat measurement data for Clay Dune repeat site \#1.

[n.d., not determined; Date, date measurements were made; Pin 1, 2, height of top of erosion pin above the ground surface; Compressive strength, vertical compressive strength of ground surface measured with a spring-piston-style pocket penetrometer; Shear stress, horizontal shear stress of ground surface measured with a hand-held shear vane; Sub-surface compaction, vertical sub-surface compaction measured in pounds per square inch (PSI) using a spear penetrometer; $\mathrm{cm}$, centimeters; $\mathrm{kg} / \mathrm{cm}^{2}$, kilograms per centimeter squared, “; inches]

\begin{tabular}{|c|c|c|c|c|c|c|c|c|c|c|c|c|}
\hline \multirow[b]{2}{*}{ Date } & \multirow{2}{*}{$\begin{array}{l}\text { Pin } 1 \\
\text { (cm) }\end{array}$} & \multirow{2}{*}{$\begin{array}{l}\text { Pin } 2 \\
(\mathrm{~cm})\end{array}$} & \multirow{2}{*}{$\begin{array}{c}\text { Compressive } \\
\text { strength } \\
\left(\mathbf{k g} / \mathrm{cm}^{2}\right)\end{array}$} & \multirow{2}{*}{$\begin{array}{c}\text { Shear } \\
\text { stress } \\
\left(\mathrm{kg} / \mathrm{cm}^{2}\right)\end{array}$} & \multicolumn{8}{|c|}{ Subsurface compaction (PSI) } \\
\hline & & & & & 3" & 6" & 9" & $12^{\prime \prime}$ & $15^{\prime \prime}$ & $18^{\prime \prime}$ & $21 "$ & $24^{\prime \prime}$ \\
\hline $8 / 18 / 2003$ & 35.0 & 30.4 & 0.42 & 2.92 & 5 & 5 & 5 & 5 & 5 & 5 & n.d. & n.d. \\
\hline $4 / 16 / 2005$ & 36.3 & 32.7 & 0.40 & 2.53 & 0 & 10 & 10 & 10 & 10 & 20 & 20 & 20 \\
\hline $5 / 26 / 2005$ & 36.9 & 32.9 & 0.33 & 0.87 & 0 & 10 & 10 & 10 & 20 & 20 & 20 & 20 \\
\hline $4 / 23 / 2006$ & 36.1 & 33.8 & 0.73 & 1.57 & 10 & 50 & 0 & 10 & 10 & 10 & 10 & 10 \\
\hline $2 / 7 / 2007$ & 37.7 & 34.0 & 1.47 & 1.51 & n.d. & n.d. & n.d. & n.d. & n.d. & n.d. & n.d. & n.d. \\
\hline $3 / 9 / 2007$ & 38.4 & 34.0 & 1.57 & 1.59 & n.d. & n.d. & n.d. & n.d. & n.d. & n.d. & n.d. & n.d. \\
\hline $4 / 21 / 2007$ & 38.4 & 33.4 & 1.23 & 1.32 & 0 & 80 & 50 & 20 & 20 & 20 & 20 & 20 \\
\hline
\end{tabular}

Table 4C. Repeat measurement data for Discovery repeat site \#1.

[n.d., not determined; Date, date measurements were made; Pin 1, 2, height of top of erosion pin above the ground surface; Compressive strength, vertical compressive strength of ground surface measured with a spring-piston-style pocket penetrometer; Shear stress, horizontal shear stress of ground surface measured with a hand-held shear vane; Sub-surface compaction, vertical sub-surface compaction measured in pounds per square inch (PSI) using a spear penetrometer; $\mathrm{cm}, \mathrm{centimeters;} \mathrm{kg} / \mathrm{cm}^{2}$, kilograms per centimeter squared; “, inches]

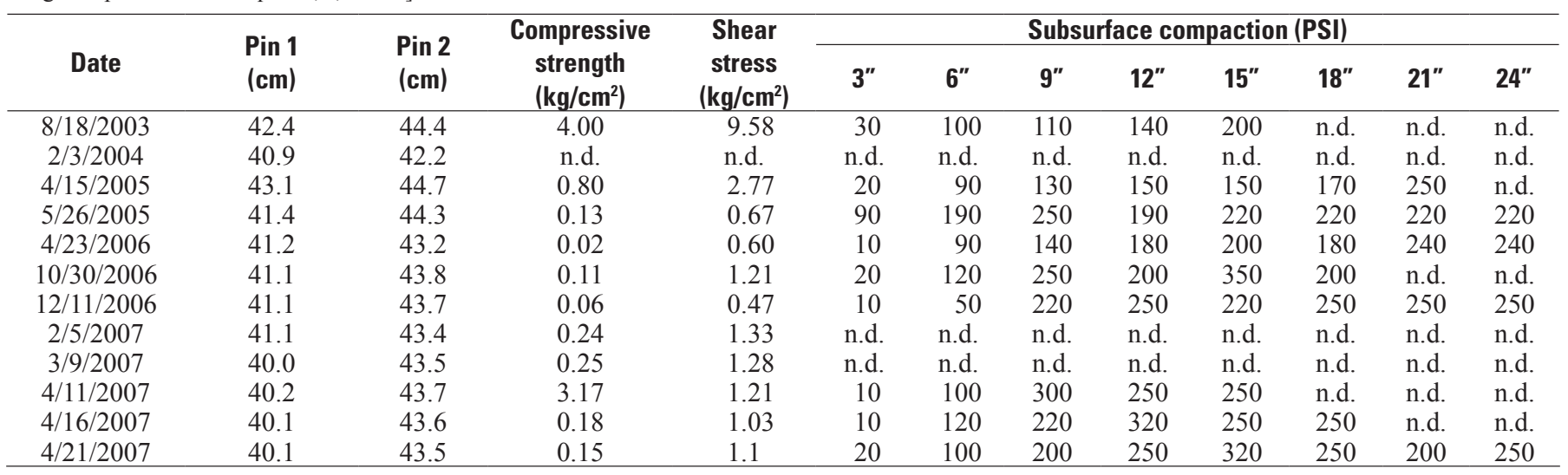


Table 4D. Repeat measurement data for East Transect repeat site \#1.

[n.d., not determined; Date, date measurements were made; Pin 1, 2, height of top of erosion pin above the ground surface; Compressive strength, vertical compressive strength of ground surface measured with a spring-piston-style pocket penetrometer; Shear stress, horizontal shear stress of ground surface measured with a hand-held shear vane; Sub-surface compaction, vertical sub-surface compaction measured in pounds per square inch (PSI) using a spear penetrometer; $\mathrm{cm}$, centimeters; $\mathrm{kg} / \mathrm{cm}^{2}$, kilograms per centimeter squared; “, inches]

\begin{tabular}{|c|c|c|c|c|c|c|c|c|c|c|c|c|}
\hline \multirow[b]{2}{*}{ Date } & \multirow{2}{*}{$\begin{array}{l}\text { Pin } 1 \\
(\mathrm{~cm})\end{array}$} & \multirow{2}{*}{$\begin{array}{l}\text { Pin } 2 \\
\text { (cm) }\end{array}$} & \multirow{2}{*}{$\begin{array}{c}\text { Compressive } \\
\text { strength } \\
\left(\mathrm{kg} / \mathrm{cm}^{2}\right)\end{array}$} & \multirow{2}{*}{$\begin{array}{c}\text { Shear } \\
\text { stress } \\
\left(\mathrm{kg} / \mathrm{cm}^{2}\right)\end{array}$} & \multicolumn{8}{|c|}{ Subsurface compaction (PSI) } \\
\hline & & & & & 3" & $6^{\prime \prime}$ & 9" & $12^{\prime \prime}$ & $15^{\prime \prime}$ & $18^{\prime \prime}$ & 21" & 24" \\
\hline $8 / 18 / 2003$ & 33.0 & 36.0 & 1.67 & 1.87 & 5 & 0 & 5 & 10 & 10 & 10 & 10 & n.d. \\
\hline $4 / 16 / 2005$ & 33.6 & 33.3 & 0.50 & 1.07 & 20 & 30 & 100 & 110 & 120 & 150 & 180 & 200 \\
\hline $5 / 26 / 2005$ & 33.2 & 33.3 & 0.63 & 1.07 & 0 & 10 & 30 & 50 & 70 & 70 & 70 & 70 \\
\hline $4 / 23 / 2006$ & 32.3 & 32.3 & 0.80 & 1.19 & 20 & 10 & 30 & 50 & 110 & 120 & 120 & 120 \\
\hline $2 / 7 / 2007$ & 31.6 & 32.7 & 2.10 & 1.23 & n.d. & n.d. & n.d. & n.d. & n.d. & n.d. & n.d. & n.d. \\
\hline $3 / 9 / 2007$ & 32.5 & 32.5 & 2.10 & 1.35 & n.d. & n.d. & n.d. & n.d. & n.d. & n.d. & n.d. & n.d. \\
\hline $4 / 10 / 2007$ & 32.1 & 32.0 & 0.23 & 1.14 & 20 & 10 & 50 & 70 & 100 & 150 & 160 & 160 \\
\hline
\end{tabular}

Table 4E. Repeat measurement data for West Transect repeat site \#1.

[n.d., not determined; Date, date measurements were made; Pin 1,2, height of top of erosion pin above the ground surface; Compressive strength, vertical compressive strength of ground surface measured with a spring-piston-style pocket penetrometer; Shear stress, horizontal shear stress of ground surface measured with a hand-held shear vane; Sub-surface compaction, vertical sub-surface compaction measured in pounds per square inch (PSI) using a spear penetrometer; $\mathrm{cm}$, centimeters; $\mathrm{kg} / \mathrm{cm}^{2}$, kilograms per centimeter squared; “, inches]

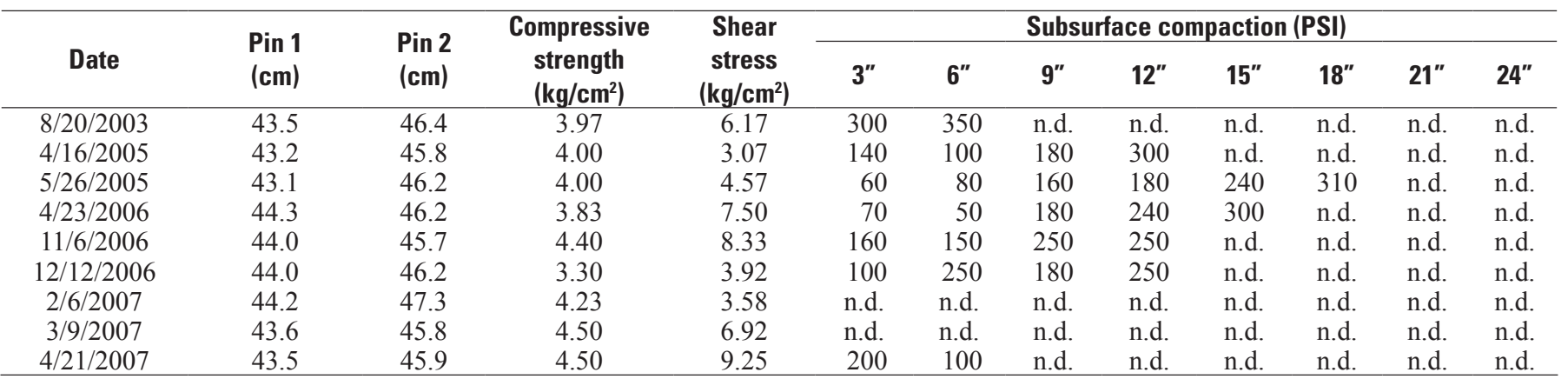

Table 4F. Repeat measurement data for Carson Slough repeat site FP-CSL.

[n.d., not determined; Date, date measurements were made; Pin 1, 2, 3, 4, height of top of erosion pin above the ground surface; M1, depth to moisture measurement \#1; M2, depth to moisture measurement \#2; >\#, depth to moisture was not reached within the depth noted, e.g. $>70$, depth of moisture was not reached within $70 \mathrm{~cm}$ of the ground surface; Conductivity, specific conductance of surface crust; Crust thickness, average measurement of 20 crusts; Compressive strength, vertical compressive strength of ground surface measured with a spring-piston-style pocket penetrometer; $\mathrm{cm}$, centimeters; $\mathrm{mS}$, millisiemens; $\mathrm{mm}, \mathrm{millimeters;} \mathrm{kg} / \mathrm{cm}^{2}, \mathrm{kilograms}$ per centimeter squared]

\begin{tabular}{|c|c|c|c|c|c|c|c|c|c|}
\hline Date & $\begin{array}{l}\text { Pin } 1 \\
(\mathrm{~cm})\end{array}$ & $\begin{array}{l}\text { Pin } 2 \\
(\mathrm{~cm})\end{array}$ & $\begin{array}{l}\text { Pin } 3 \\
(\mathrm{~cm})\end{array}$ & $\begin{array}{l}\text { Pin } 4 \\
\text { (cm) }\end{array}$ & $\begin{array}{c}\text { Depth to } \\
\text { moisture M1 } \\
\text { (cm) }\end{array}$ & $\begin{array}{c}\text { Depth to } \\
\text { moisture M2 } \\
\text { (cm) }\end{array}$ & $\begin{array}{c}\text { Conductivity } \\
\text { (mS) }\end{array}$ & $\begin{array}{c}\text { Crust } \\
\text { thickness } \\
(\mathrm{mm})\end{array}$ & $\begin{array}{c}\text { Compressive } \\
\text { strength } \\
\left(\mathrm{kg} / \mathrm{cm}^{2}\right)\end{array}$ \\
\hline $12 / 15 / 2006$ & 45.7 & 46.4 & 46.2 & 48.9 & 9.00 & 4.00 & 3.70 & 3.2 & 0.474 \\
\hline $3 / 7 / 2007$ & 46.0 & 47.0 & 45.5 & 49.0 & 3.00 & 5.00 & 0.46 & 7.9 & 0.053 \\
\hline $4 / 21 / 2007$ & 45.7 & 47.1 & 45.4 & 48.1 & 6.00 & 7.00 & 6.20 & 6.4 & 1.505 \\
\hline $5 / 31 / 2007$ & 45.5 & 47.0 & 45.7 & 48.0 & 7.00 & 9.00 & 5.90 & 9.0 & 0.044 \\
\hline $11 / 14 / 2007$ & 47.5 & 47.5 & 48.0 & 51.5 & 5.00 & 5.00 & 20.00 & 3.0 & 0.096 \\
\hline $4 / 8 / 2008$ & 46.0 & 46.5 & 45.5 & 50.5 & 0.50 & 0.50 & 26.00 & 9.0 & 0.020 \\
\hline $5 / 20 / 2008$ & n.d. & n.d. & n.d. & n.d. & n.d. & n.d. & 27.90 & n.d. & n.d. \\
\hline $10 / 15 / 2008$ & n.d. & n.d. & n.d. & n.d. & n.d. & n.d. & 26.90 & n.d. & n.d. \\
\hline $11 / 24 / 2008$ & 44.0 & 46.3 & 44.8 & 48.8 & 9.00 & 5.00 & 15.80 & 8.9 & 0.083 \\
\hline $1 / 2 / 2009$ & 44.9 & 45.0 & 44.6 & 46.9 & 3.00 & 2.00 & 9.60 & 7.4 & 0.012 \\
\hline $2 / 26 / 2009$ & 47.5 & 48.0 & 46.0 & 50.0 & 0.00 & 0.00 & 15.90 & 8.7 & 0.002 \\
\hline
\end{tabular}


Table 4G. Repeat measurement data for Clay Dune repeat site FP-CDN.

[n.d., not determined; Date, date measurements were made; Pin 1, 2, 3, 4, height of top of erosion pin above the ground surface; M1, depth to moisture measurement \#1; M2, depth to moisture measurement \#2; >\#, depth to moisture was not reached within the depth noted, e.g. $>70$, depth of moisture was not reached within $70 \mathrm{~cm}$ of the ground surface; Conductivity, specific conductance of surface crust; Crust thickness, average measurement of 20 crusts; Compressive strength, vertical compressive strength of ground surface measured with a spring-piston-style pocket penetrometer; $\mathrm{cm}$, centimeters; $\mathrm{mS}$, millisiemens; mm, millimeters; $\mathrm{kg} / \mathrm{cm}^{2}, \mathrm{kilograms}$ per centimeter squared]

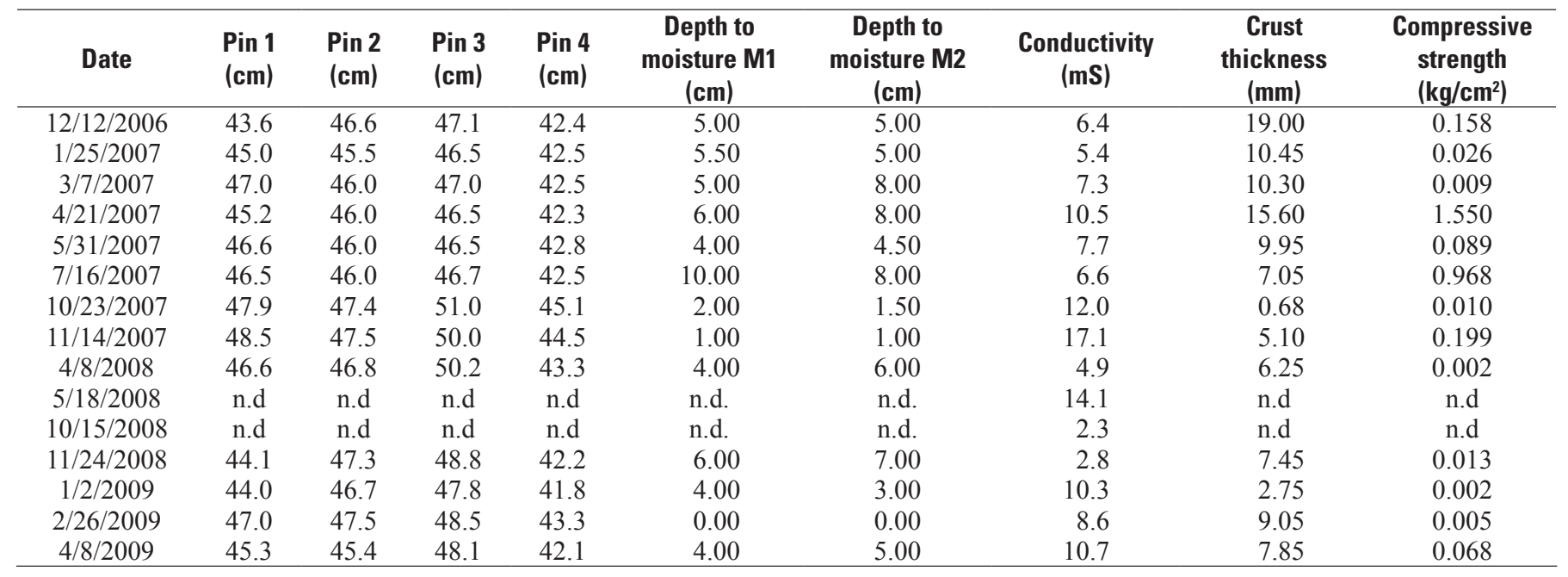

Table 4H. Repeat measurement data for Discovery repeat site FP-DSC.

[n.d., not determined; Date, date measurements were made; Pin 1, 2, 3, 4, height of top of erosion pin above the ground surface; M1, depth to moisture measurement \#1; M2, depth to moisture measurement $\# 2$; $>\#$, depth to moisture was not reached within the depth noted, e.g. $>70$, depth of moisture was not reached within $70 \mathrm{~cm}$ of the ground surface; Conductivity, specific conductance of surface crust; Crust thickness, average measurement of 20 crusts; Compressive strength, vertical compressive strength of ground surface measured with a spring-piston-style pocket penetrometer; $\mathrm{cm}$, centimeters; $\mathrm{mS}$, millisiemens; $\mathrm{mm}$, millimeters; $\mathrm{kg} / \mathrm{cm}^{2}, \mathrm{kilograms} \mathrm{per}$ centimeter squared]

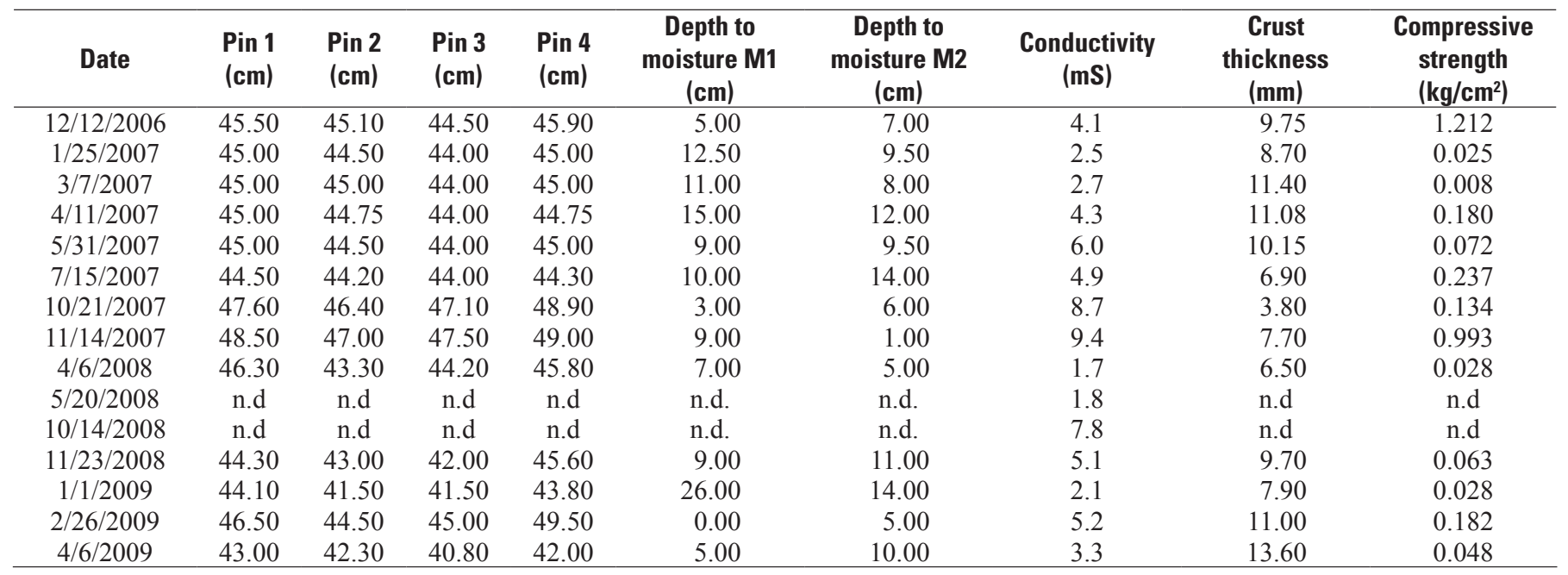


Table 4I. Repeat measurement data for East Transect FP-ETN.

[n.d., not determined; Date, date measurements were made; Pin 1, 2, 3, 4, height of top of erosion pin above the ground surface; M1, depth to moisture measurement \#1; M2, depth to moisture measurement $\# 2 ;>\#$, depth to moisture was not reached within the depth noted, e.g. $>70$, depth of moisture was not reached within $70 \mathrm{~cm}$ of the ground surface; Conductivity, specific conductance of surface crust; Crust thickness, average measurement of 20 crusts; Compressive strength, vertical compressive strength of ground surface measured with a spring-piston-style pocket penetrometer; $\mathrm{cm}$, centimeters; $\mathrm{mS}$, millisiemens; $\mathrm{mm}$, millimeters; $\mathrm{kg} / \mathrm{cm}^{2}, \mathrm{kilograms}$ per centimeter squared]

\begin{tabular}{|c|c|c|c|c|c|c|c|c|c|}
\hline Date & $\begin{array}{l}\text { Pin } 1 \\
(\mathrm{~cm})\end{array}$ & $\begin{array}{l}\text { Pin } 2 \\
(\mathrm{~cm})\end{array}$ & $\begin{array}{l}\text { Pin } 3 \\
(\mathrm{~cm})\end{array}$ & $\begin{array}{l}\text { Pin } 4 \\
(\mathrm{~cm})\end{array}$ & $\begin{array}{l}\text { Depth to } \\
\text { moisture M1 } \\
\text { (cm) }\end{array}$ & $\begin{array}{c}\text { Depth to } \\
\text { moisture M2 } \\
\text { (cm) }\end{array}$ & $\begin{array}{c}\text { Conductivity } \\
\text { (mS) }\end{array}$ & $\begin{array}{c}\text { Crust } \\
\text { thickness } \\
(\mathrm{mm})\end{array}$ & $\begin{array}{c}\text { Compressive } \\
\text { strength } \\
\left(\mathrm{kg} / \mathrm{cm}^{2}\right)\end{array}$ \\
\hline $12 / 12 / 2006$ & 45.9 & 46.2 & 45.6 & 44.7 & 38.1 & 27.9 & 0.69 & 23.40 & 0.856 \\
\hline $3 / 7 / 2007$ & 46.5 & 46.5 & 46.0 & 45.0 & 23 & 24 & 0.49 & 20.45 & 0.725 \\
\hline $4 / 11 / 2007$ & 49.0 & 48.5 & 48.0 & 46.0 & 28 & 28 & 0.55 & 17.25 & 1.665 \\
\hline $5 / 31 / 2007$ & 48.9 & 49.4 & 48.0 & 47.0 & 32 & 28 & 0.53 & 17.25 & 1.258 \\
\hline $11 / 14 / 2007$ & 50.0 & 50.5 & 48.0 & 47.0 & 12 & 10 & 1.58 & 18.90 & 0.857 \\
\hline $4 / 8 / 2008$ & 48.8 & 48.1 & 48.8 & 46.3 & 12 & 16 & 0.37 & 14.30 & 0.078 \\
\hline $5 / 20 / 2008$ & n.d. & n.d. & n.d. & n.d. & n.d. & n.d. & 0.47 & n.d. & n.d. \\
\hline $10 / 15 / 2008$ & n.d. & n.d. & n.d. & n.d. & n.d. & n.d. & 0.48 & n.d. & n.d. \\
\hline $11 / 23 / 2008$ & 49.5 & 48.0 & 48.8 & 46.5 & 41 & 30 & 0.57 & 15.65 & 0.057 \\
\hline $1 / 2 / 2009$ & 47.4 & 44.5 & 47.5 & 46.0 & $>50$ & $>50$ & 0.36 & 14.85 & 0.033 \\
\hline
\end{tabular}

Table 4J. Repeat measurement data for West Transect repeat site FP-WTN.

[n.d., not determined; Date, date measurements were made; Pin 1, 2, 3, 4, height of top of erosion pin above the ground surface; M1, depth to moisture measurement \#1; M2, depth to moisture measurement \#2; >\#, depth to moisture was not reached within the depth noted, e.g. $>70$, depth of moisture was not reached within $70 \mathrm{~cm}$ of the ground surface; Conductivity, specific conductance of surface crust; Crust thickness, average measurement of 20 crusts; Compressive strength, vertical compressive strength of ground surface measured with a spring-piston-style pocket penetrometer; $\mathrm{cm}$, centimeters; $\mathrm{mS}$, millisiemens; $\mathrm{mm}$, millimeters; $\mathrm{kg} / \mathrm{cm}^{2}$, kilograms per centimeter squared]

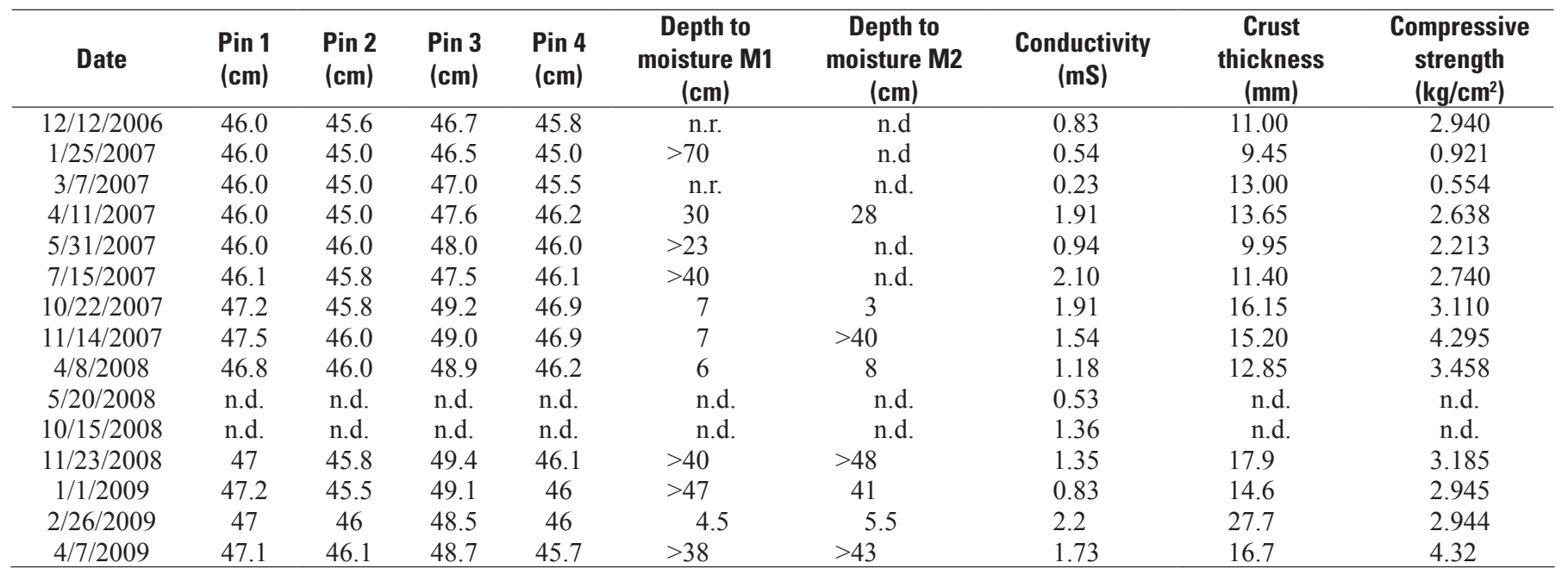


Table 5. Casing, water level, well depth, and ground-surface elevations for Franklin Lake playa wells. All measurements except ground-surface elevation were made in feet and were later converted to meters.

[Well ID, unique well identifier; Casing height, height to the top of the casing from the ground surface; Water level, the depth of water below the ground surface; Total well depth, the depth to the bottom of the well below the ground surface; Ground-surface elevation, elevation determined using a GPS receiver and post correction with an average vertical error of $20 \mathrm{~cm}$; ft, feet; $\mathrm{m}$, meters; n.d., not determined]

\begin{tabular}{|c|c|c|c|c|c|c|c|c|}
\hline Well ID & $\begin{array}{c}\text { Date } \\
\text { measured }\end{array}$ & $\begin{array}{l}\text { Casing } \\
\text { height } \\
\text { (ft) }\end{array}$ & $\begin{array}{c}\text { Casing } \\
\text { height } \\
\text { (m) }\end{array}$ & $\begin{array}{c}\text { Water } \\
\text { level } \\
\text { (ft) }\end{array}$ & $\begin{array}{c}\text { Water } \\
\text { level } \\
(\mathrm{m})\end{array}$ & $\begin{array}{c}\text { Total well } \\
\text { depth } \\
\text { (ft) }\end{array}$ & $\begin{array}{c}\text { Total well } \\
\text { depth } \\
(\mathrm{m})\end{array}$ & $\begin{array}{c}\text { Ground-surface } \\
\text { elevation } \\
\text { (m) }\end{array}$ \\
\hline GS-1 & $\begin{array}{c}2 / 7 / 2007 \\
3 / 9 / 2007 \\
4 / 11 / 2007 \\
7 / 16 / 2007 \\
10 / 23 / 2007 \\
11 / 17 / 2007 \\
4 / 11 / 2008 \\
11 / 24 / 2008 \\
1 / 2 / 2009 \\
2 / 23 / 2009 \\
4 / 8 / 2009\end{array}$ & 4.08 & 1.24 & $\begin{array}{l}1.72 \\
1.72 \\
1.72 \\
2.12 \\
1.52 \\
1.57 \\
1.52 \\
2.12 \\
2.17 \\
2.02 \\
1.87\end{array}$ & $\begin{array}{l}0.52 \\
0.52 \\
0.52 \\
0.65 \\
0.46 \\
0.48 \\
0.46 \\
0.65 \\
0.66 \\
0.62 \\
0.57\end{array}$ & 6.52 & 1.99 & 611.68 \\
\hline GS-4 & $\begin{array}{c}11 / 6 / 2006 \\
11 / 8 / 2006 \\
12 / 13 / 2006 \\
2 / 7 / 2007 \\
3 / 9 / 2007 \\
4 / 21 / 2007 \\
7 / 16 / 2007 \\
10 / 23 / 2007 \\
11 / 17 / 2007 \\
4 / 11 / 2008 \\
11 / 24 / 2008 \\
1 / 2 / 2009 \\
2 / 23 / 2009 \\
4 / 8 / 2009\end{array}$ & 3.60 & 1.10 & $\begin{array}{l}4.50 \\
4.30 \\
4.70 \\
4.70 \\
4.63 \\
4.60 \\
4.60 \\
4.70 \\
4.73 \\
4.65 \\
4.90 \\
4.95 \\
4.95 \\
4.85\end{array}$ & $\begin{array}{l}1.37 \\
1.31 \\
1.43 \\
1.43 \\
1.41 \\
1.40 \\
1.40 \\
1.43 \\
1.44 \\
1.42 \\
1.49 \\
1.51 \\
1.51 \\
1.48\end{array}$ & 22.80 & 6.95 & 611.39 \\
\hline GS-12 & $\begin{array}{c}2 / 7 / 2007 \\
3 / 9 / 2007 \\
4 / 15 / 2007 \\
7 / 16 / 2007 \\
10 / 23 / 2007 \\
11 / 17 / 2007 \\
4 / 11 / 2008 \\
11 / 24 / 2008 \\
1 / 2 / 2009 \\
2 / 23 / 2009 \\
4 / 8 / 2009\end{array}$ & 1.29 & 0.39 & $\begin{array}{l}9.51 \\
9.45 \\
9.46 \\
9.51 \\
8.61 \\
8.59 \\
8.66 \\
9.41 \\
9.46 \\
9.46 \\
9.56\end{array}$ & $\begin{array}{l}2.90 \\
2.88 \\
2.88 \\
2.90 \\
2.62 \\
2.62 \\
2.64 \\
2.87 \\
2.88 \\
2.88 \\
2.91\end{array}$ & 28.91 & 8.81 & 612.82 \\
\hline GS-15 & $\begin{array}{c}11 / 3 / 2006 \\
11 / 8 / 2006 \text { (a.m.) } \\
11 / 8 / 2006 \text { (p.m.) } \\
12 / 13 / 2006 \\
2 / 7 / 2007 \\
3 / 9 / 2007 \\
4 / 21 / 2007 \\
7 / 15 / 2007 \\
10 / 22 / 2007 \\
11 / 17 / 2007 \\
4 / 11 / 2008 \\
11 / 23 / 2008 \\
1 / 1 / 2009 \\
2 / 23 / 2009 \\
4 / 7 / 2009\end{array}$ & 2.83 & 0.86 & $\begin{array}{l}7.57 \\
7.57 \\
7.87 \\
7.77 \\
7.87 \\
7.87 \\
7.97 \\
8.07 \\
7.97 \\
8.02 \\
7.82 \\
7.72 \\
7.82 \\
7.97 \\
7.97\end{array}$ & $\begin{array}{l}2.31 \\
2.31 \\
2.40 \\
2.37 \\
2.40 \\
2.40 \\
2.43 \\
2.46 \\
2.43 \\
2.44 \\
2.38 \\
2.35 \\
2.38 \\
2.43 \\
2.43\end{array}$ & 23.57 & 7.18 & 610.41 \\
\hline
\end{tabular}


Table 5. Casing, water level, well depth, and ground-surface elevations for Franklin Lake playa wells. All measurements except ground-surface elevation were made in feet and were later converted to meters.-Continued

[Well ID, unique well identifier; Casing height, height to the top of the casing from the ground surface; Water level, the depth of water below the ground surface; Total well depth, the depth to the bottom of the well below the ground surface; Ground-surface elevation, elevation determined using a GPS receiver and post correction with an average vertical error of $20 \mathrm{~cm}$; ft, feet; m, meters; n.d., not determined]

\begin{tabular}{|c|c|c|c|c|c|c|c|c|}
\hline Well ID & $\begin{array}{c}\text { Date } \\
\text { measured }\end{array}$ & $\begin{array}{c}\text { Casing } \\
\text { height } \\
\text { (ft) }\end{array}$ & $\begin{array}{c}\text { Casing } \\
\text { height } \\
\text { (m) }\end{array}$ & $\begin{array}{c}\text { Water } \\
\text { level } \\
\text { (ft) }\end{array}$ & $\begin{array}{c}\text { Water } \\
\text { level } \\
(\mathrm{m})\end{array}$ & $\begin{array}{c}\text { Total well } \\
\text { depth } \\
\text { (ft) }\end{array}$ & $\begin{array}{c}\text { Total well } \\
\text { depth } \\
(\mathrm{m})\end{array}$ & $\begin{array}{l}\text { Ground-surface } \\
\text { elevation } \\
\text { (m) }\end{array}$ \\
\hline GS-18 & $\begin{array}{c}11 / 3 / 2006 \\
12 / 12 / 2006 \\
2 / 7 / 2007 \\
3 / 9 / 2007 \\
4 / 16 / 2007 \\
7 / 15 / 2007 \\
10 / 22 / 2007 \\
11 / 17 / 2007 \\
4 / 11 / 2008 \\
11 / 23 / 2008 \\
1 / 1 / 2009 \\
2 / 23 / 2009 \\
4 / 7 / 2009\end{array}$ & 3.33 & 1.01 & $\begin{array}{r}9.97 \\
10.37 \\
10.42 \\
10.42 \\
10.67 \\
10.57 \\
10.42 \\
10.40 \\
10.72 \\
10.47 \\
10.52 \\
10.62 \\
10.72\end{array}$ & $\begin{array}{l}3.04 \\
3.16 \\
3.18 \\
3.18 \\
3.25 \\
3.22 \\
3.18 \\
3.17 \\
3.27 \\
3.19 \\
3.21 \\
3.24 \\
3.27\end{array}$ & 26.87 & 8.19 & 610.52 \\
\hline Chemical Well (Cz1-middle) & $\begin{array}{c}11 / 6 / 2006 \\
11 / 7 / 2006 \\
12 / 13 / 2006 \\
2 / 6 / 2007 \\
3 / 9 / 2007 \\
4 / 21 / 2007 \\
7 / 15 / 2007 \\
10 / 22 / 2007 \\
11 / 17 / 2007 \\
4 / 11 / 2008 \\
11 / 23 / 2008 \\
1 / 1 / 2009 \\
2 / 21 / 2009 \\
4 / 6 / 2009\end{array}$ & 2.40 & 0.73 & $\begin{array}{r}8.10 \\
12.50 \\
8.30 \\
8.30 \\
8.20 \\
8.15 \\
8.20 \\
8.20 \\
8.10 \\
7.95 \\
8.00 \\
8.00 \\
8.05 \\
8.05\end{array}$ & $\begin{array}{l}2.47 \\
3.81 \\
2.53 \\
2.53 \\
2.50 \\
2.48 \\
2.50 \\
2.50 \\
2.47 \\
2.42 \\
2.44 \\
2.44 \\
2.45 \\
2.45\end{array}$ & 25.50 & 7.77 & 609.47 \\
\hline Chemical Well (Cz1a-south) & $\begin{array}{c}11 / 6 / 2006 \\
12 / 13 / 2006 \\
2 / 6 / 2007 \\
3 / 9 / 2007 \\
4 / 21 / 2007 \\
7 / 15 / 2007 \\
10 / 22 / 2007 \\
11 / 17 / 2007 \\
4 / 11 / 2008 \\
11 / 23 / 2008 \\
1 / 1 / 2009 \\
2 / 21 / 2009 \\
4 / 6 / 2009\end{array}$ & 1.50 & 0.46 & $\begin{array}{l}6.30 \\
8.20 \\
8.00 \\
8.00 \\
8.10 \\
8.10 \\
8.40 \\
8.08 \\
8.15 \\
8.00 \\
7.95 \\
8.00 \\
8.10\end{array}$ & $\begin{array}{l}1.92 \\
2.50 \\
2.44 \\
2.44 \\
2.47 \\
2.47 \\
2.56 \\
2.46 \\
2.48 \\
2.44 \\
2.42 \\
2.44 \\
2.47\end{array}$ & 86.90 & 26.49 & n.d. \\
\hline
\end{tabular}


Table 5. Casing, water level, well depth, and ground-surface elevations for Franklin Lake playa wells. All measurements except ground-surface elevation were made in feet and were later converted to meters.-Continued

[Well ID, unique well identifier; Casing height, height to the top of the casing from the ground surface; Water level, the depth of water below the ground surface; Total well depth, the depth to the bottom of the well below the ground surface; Ground-surface elevation, elevation determined using a GPS receiver and post correction with an average vertical error of $20 \mathrm{~cm}$; ft, feet; m, meters; n.d., not determined]

\begin{tabular}{|c|c|c|c|c|c|c|c|c|}
\hline Well ID & $\begin{array}{c}\text { Date } \\
\text { measured }\end{array}$ & $\begin{array}{c}\text { Casing } \\
\text { height } \\
\text { (ft) }\end{array}$ & $\begin{array}{l}\text { Casing } \\
\text { height } \\
\text { (m) }\end{array}$ & $\begin{array}{l}\text { Water } \\
\text { level } \\
\text { (ft) }\end{array}$ & $\begin{array}{c}\text { Water } \\
\text { level } \\
(\mathrm{m})\end{array}$ & $\begin{array}{c}\text { Total well } \\
\text { depth } \\
\text { (ft) }\end{array}$ & $\begin{array}{l}\text { Total well } \\
\text { depth } \\
\text { (m) }\end{array}$ & $\begin{array}{c}\text { Ground-surface } \\
\text { elevation } \\
\text { (m) }\end{array}$ \\
\hline $\mathrm{Cz} 5$ & $\begin{array}{c}12 / 13 / 2006 \\
2 / 6 / 2007 \\
3 / 9 / 2007 \\
4 / 21 / 2007 \\
7 / 15 / 2007 \\
10 / 22 / 2007 \\
11 / 17 / 2007 \\
4 / 11 / 2008 \\
11 / 23 / 2008 \\
1 / 1 / 2009 \\
2 / 21 / 2009 \\
4 / 6 / 2009\end{array}$ & 0.90 & 0.27 & $\begin{array}{l}7.00 \\
6.90 \\
6.90 \\
6.95 \\
7.00 \\
7.15 \\
6.90 \\
6.85 \\
6.85 \\
6.75 \\
6.80 \\
6.90\end{array}$ & $\begin{array}{l}2.13 \\
2.10 \\
2.10 \\
2.12 \\
2.13 \\
2.18 \\
2.10 \\
2.09 \\
2.09 \\
2.06 \\
2.07 \\
2.10\end{array}$ & 34.30 & 10.45 & n.d. \\
\hline Cz10 & $\begin{array}{c}2 / 6 / 2007 \\
3 / 9 / 2007 \\
4 / 21 / 2007 \\
7 / 15 / 2007 \\
10 / 22 / 2007 \\
11 / 17 / 2007 \\
4 / 11 / 2008 \\
11 / 23 / 2008 \\
1 / 1 / 2009 \\
2 / 21 / 2009 \\
4 / 6 / 2009\end{array}$ & 0.96 & 0.29 & $\begin{array}{l}9.54 \\
9.44 \\
9.44 \\
9.54 \\
9.59 \\
9.59 \\
9.39 \\
9.54 \\
9.59 \\
9.54 \\
9.59\end{array}$ & $\begin{array}{l}2.91 \\
2.88 \\
2.88 \\
2.91 \\
2.92 \\
2.92 \\
2.86 \\
2.91 \\
2.92 \\
2.91 \\
2.92\end{array}$ & 31.04 & 9.46 & n.d. \\
\hline Cz11 & $\begin{array}{c}2 / 6 / 2007 \\
3 / 9 / 2007 \\
4 / 16 / 2007 \\
7 / 15 / 2007 \\
10 / 21 / 2007 \\
11 / 17 / 2007 \\
4 / 9 / 2008 \\
11 / 23 / 2008 \\
1 / 1 / 2009 \\
2 / 21 / 2009 \\
4 / 6 / 2009\end{array}$ & 0.90 & 0.27 & $\begin{array}{l}13.00 \\
12.95 \\
13.00 \\
15.55 \\
14.60 \\
14.35 \\
13.75 \\
13.00 \\
13.10 \\
13.10 \\
13.10\end{array}$ & $\begin{array}{l}3.96 \\
3.95 \\
3.96 \\
4.74 \\
4.45 \\
4.37 \\
4.19 \\
3.96 \\
3.99 \\
3.99 \\
3.99\end{array}$ & 17.30 & 5.27 & n.d. \\
\hline Bucket Well (Cz13) & $\begin{array}{c}11 / 3 / 2006 \\
12 / 11 / 2006 \\
2 / 6 / 2007 \\
3 / 9 / 2007 \\
4 / 21 / 2007 \\
7 / 15 / 2007 \\
10 / 22 / 2007 \\
11 / 17 / 2007 \\
4 / 9 / 2008 \\
11 / 22 / 2008 \\
1 / 1 / 2009 \\
2 / 22 / 2009 \\
4 / 7 / 2009\end{array}$ & 3.50 & 1.07 & $\begin{array}{l}11.70 \\
11.20 \\
11.30 \\
11.25 \\
11.30 \\
11.50 \\
11.20 \\
11.25 \\
11.35 \\
11.35 \\
11.40 \\
11.45 \\
11.45\end{array}$ & $\begin{array}{l}3.57 \\
3.41 \\
3.44 \\
3.43 \\
3.44 \\
3.51 \\
3.41 \\
3.43 \\
3.46 \\
3.46 \\
3.47 \\
3.49 \\
3.49\end{array}$ & 15.20 & 4.63 & 610.13 \\
\hline
\end{tabular}


Table 5. Casing, water level, well depth, and ground-surface elevations for Franklin Lake playa wells. All measurements except ground-surface elevation were made in feet and were later converted to meters. - Continued

[Well ID, unique well identifier; Casing height, height to the top of the casing from the ground surface; Water level, the depth of water below the ground surface; Total well depth, the depth to the bottom of the well below the ground surface; Ground-surface elevation, elevation determined using a GPS receiver and post correction with an average vertical error of $20 \mathrm{~cm}$; ft, feet; m, meters; n.d., not determined]

\begin{tabular}{|c|c|c|c|c|c|c|c|c|}
\hline Well ID & $\begin{array}{c}\text { Date } \\
\text { measured }\end{array}$ & $\begin{array}{c}\text { Casing } \\
\text { height } \\
\text { (ft) }\end{array}$ & $\begin{array}{c}\text { Casing } \\
\text { height } \\
\text { (m) }\end{array}$ & $\begin{array}{c}\text { Water } \\
\text { level } \\
\text { (ft) }\end{array}$ & $\begin{array}{c}\text { Water } \\
\text { level } \\
(\mathrm{m})\end{array}$ & $\begin{array}{c}\text { Total well } \\
\text { depth } \\
\text { (ft) }\end{array}$ & $\begin{array}{c}\text { Total well } \\
\text { depth } \\
\text { (m) }\end{array}$ & $\begin{array}{c}\text { Ground-surface } \\
\text { elevation } \\
\text { (m) }\end{array}$ \\
\hline $\mathrm{Cz} 14$ & $\begin{array}{c}2 / 6 / 2007 \\
3 / 9 / 2007 \\
4 / 21 / 2007 \\
7 / 15 / 2007 \\
10 / 21 / 2007 \\
11 / 17 / 2007 \\
4 / 9 / 2008 \\
11 / 22 / 2008 \\
1 / 1 / 2009 \\
2 / 21 / 2009 \\
4 / 6 / 2009\end{array}$ & 1.30 & 0.40 & $\begin{array}{l}4.50 \\
4.50 \\
4.40 \\
4.50 \\
4.50 \\
4.45 \\
4.35 \\
4.45 \\
4.45 \\
4.45 \\
4.50\end{array}$ & $\begin{array}{l}1.37 \\
1.37 \\
1.34 \\
1.37 \\
1.37 \\
1.36 \\
1.33 \\
1.36 \\
1.36 \\
1.36 \\
1.37\end{array}$ & 58.10 & 17.10 & 610.34 \\
\hline USGS North Well - Shallow & $\begin{array}{l}4 / 11 / 2008 \\
2 / 24 / 2009\end{array}$ & 0.20 & 0.06 & $\begin{array}{l}8.65 \\
8.95\end{array}$ & $\begin{array}{l}2.64 \\
2.73\end{array}$ & 41.00 & 12.50 & 609.46 \\
\hline USGS South Well - Shallow & $\begin{array}{l}4 / 11 / 2008 \\
2 / 27 / 2009\end{array}$ & 0.20 & 0.06 & $\begin{array}{l}12.60 \\
12.90\end{array}$ & 3.84 & 36.80 & 11.22 & 609.12 \\
\hline USGS South Well - Deep & $\begin{array}{l}4 / 11 / 2008 \\
2 / 27 / 2009\end{array}$ & 0.20 & 0.06 & $\begin{array}{l}12.65 \\
12.95\end{array}$ & 3.86 & 106.90 & 32.58 & 609.12 \\
\hline Metal Well North & $\begin{array}{c}4 / 11 / 2008 \\
11 / 22 / 2008 \\
1 / 1 / 2009 \\
2 / 21 / 2009 \\
4 / 6 / 2009\end{array}$ & 2.20 & 0.67 & $\begin{array}{l}16.30 \\
17.20 \\
17.15 \\
17.25 \\
17.35\end{array}$ & $\begin{array}{l}4.97 \\
5.24 \\
5.23 \\
5.26 \\
5.29\end{array}$ & 130.60 & 39.81 & 608.39 \\
\hline Metal Well South & $\begin{array}{c}4 / 11 / 2008 \\
11 / 22 / 2008 \\
1 / 1 / 2009 \\
2 / 21 / 2009 \\
4 / 6 / 2009\end{array}$ & 0.80 & 0.24 & $\begin{array}{l}17.20 \\
18.05 \\
18.10 \\
18.15 \\
18.25 \\
\end{array}$ & $\begin{array}{l}5.24 \\
5.50 \\
5.52 \\
5.53 \\
5.56 \\
\end{array}$ & 123.00 & 37.49 & 608.25 \\
\hline
\end{tabular}


Table 6. Values of field parameters measured on water samples.

[Sample site, name of sampling site; Sample ID, unique sample identifier; n.d., not determined; *, well incompletely purged prior to sampling; ${ }^{\circ} \mathrm{C}$, degrees Celsius; $\mathrm{mS} \mathrm{cm}{ }^{-1}$, millisiemens per centimeter, $\mathrm{mg} / \mathrm{L} \mathrm{CaCO}_{3}$, milligrams per liter calcium carbonate]

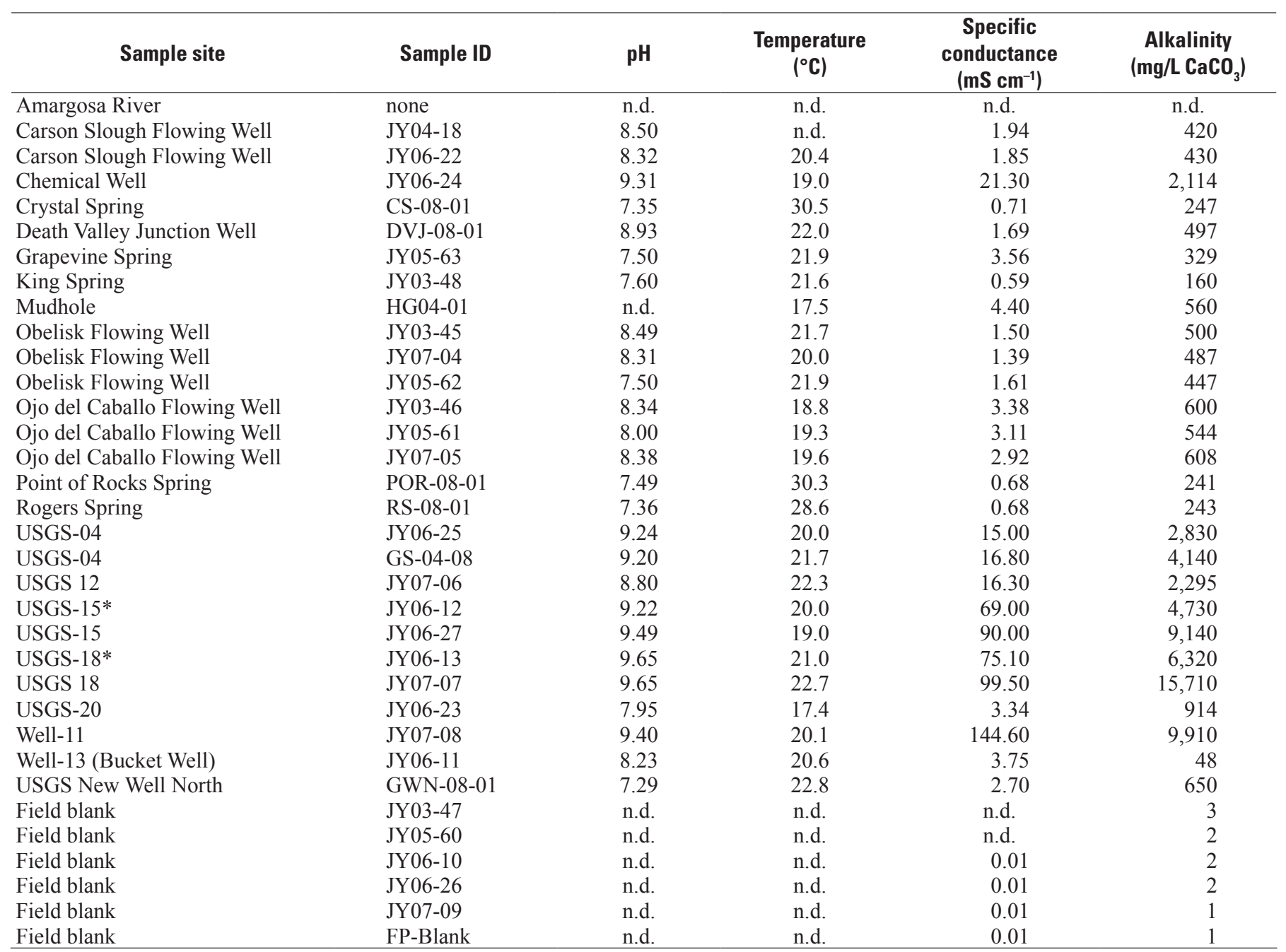


Table 7. Moisture content of sediment collected by augering at Franklin Lake playa.

[Sample site, name of sampling site; Sample ID, unique sample identifier; Gravimetric water content, determined by weight loss on heating at $105^{\circ} \mathrm{C}$; Adsorbed water, the amount of water adsorbed by the dried sample in an atmosphere of $100 \%$ humidity; n.d., not determined; cm, centimeters; wt. $\%$, weight percent]

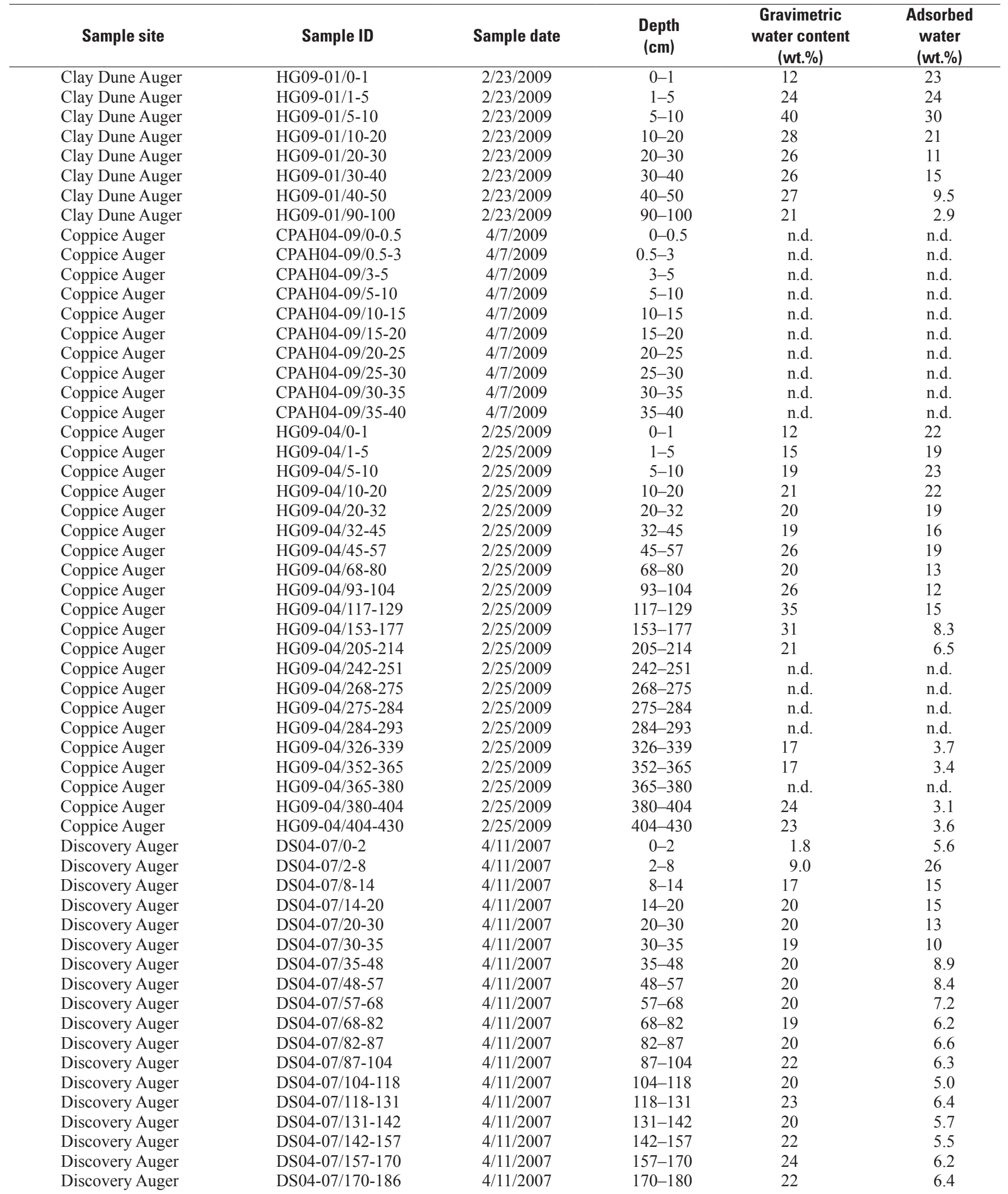


Table 7. Moisture content of sediment collected by augering at Franklin Lake playa._Continued

[Sample site, name of sampling site; Sample ID, unique sample identifier; Gravimetric water content, determined by weight loss on heating at $105^{\circ} \mathrm{C}$; Adsorbed water, the amount of water adsorbed by the dried sample in an atmosphere of $100 \%$ humidity; n.d., not determined; cm, centimeters; wt.\%, weight percent]

\begin{tabular}{|c|c|c|c|c|c|}
\hline Sample site & Sample ID & Sample date & $\begin{array}{c}\text { Depth } \\
\text { (cm) }\end{array}$ & $\begin{array}{c}\text { Gravimetric } \\
\text { water content } \\
\text { (wt. } \% \text { ) }\end{array}$ & $\begin{array}{c}\text { Adsorbed } \\
\text { water } \\
\text { (wt.\%) }\end{array}$ \\
\hline Discovery Auger & DS04-07b/0-1 & $4 / 16 / 2007$ & $0-1$ & 16 & 22 \\
\hline Discovery Auger & DS04-07b/2.5-5 & $4 / 16 / 2007$ & $2.5-5$ & 5.5 & 25 \\
\hline Discovery Auger & HG09-02/0-1 & $2 / 24 / 2009$ & $0-1$ & 5.6 & 17 \\
\hline Discovery Auger & HG09-02/1-5 & $2 / 24 / 2009$ & $1-5$ & 19 & 25 \\
\hline Discovery Auger & HG09-02/20-26 & $2 / 24 / 2009$ & $20-26$ & 22 & 18 \\
\hline Discovery Auger & HG09-02/37-48 & $2 / 24 / 2009$ & $37-48$ & 25 & 11 \\
\hline Discovery Auger & HG09-02/59-70 & $2 / 24 / 2009$ & $59-70$ & 23 & 6.8 \\
\hline Discovery Auger & HG09-02/80-89 & $2 / 24 / 2009$ & $80-89$ & 24 & 6.7 \\
\hline Discovery Auger & HG09-02/98-110 & $2 / 24 / 2009$ & $98-110$ & 22 & 6.8 \\
\hline East Transect Auger & FPAH06-08/0-1 & $6 / 24 / 2008$ & $0-1$ & 1.47 & 8.5 \\
\hline East Transect Auger & FPAH06-08/20-25 & $6 / 24 / 2008$ & $20-25$ & 14 & 22 \\
\hline East Transect Auger & FPAH06-08/25-30 & $6 / 24 / 2008$ & $25-30$ & 17 & 25 \\
\hline East Transect Auger & FPAH06-08/30-35 & $6 / 24 / 2008$ & $30-35$ & 17 & 24 \\
\hline East Transect Auger & FPAH06-08/35-40 & $6 / 24 / 2008$ & $35-40$ & 17 & 24 \\
\hline East Transect Auger & FPAH06-08/40-45 & $6 / 24 / 2008$ & $40-45$ & 18 & 25 \\
\hline East Transect Auger & FPAH06-08/45-48 & $6 / 24 / 2008$ & $45-48$ & 17 & 25 \\
\hline East Transect Auger & FPAH06-08/48-52 & $6 / 24 / 2008$ & $48-52$ & 18 & 26 \\
\hline East Transect Auger & FPAH02-09/0-1 & $2 / 25 / 2009$ & $0-1$ & 3.4 & 6.9 \\
\hline East Transect Auger & FPAH02-09/1-2.5 & $2 / 25 / 2009$ & $1-2.5$ & 8.1 & 8.9 \\
\hline East Transect Auger & FPAH02-09/2.5-4 & $2 / 25 / 2009$ & $2.5-4$ & 23 & 21 \\
\hline East Transect Auger & FPAH02-09/4-10 & $2 / 25 / 2009$ & 4-10 & 12 & 17 \\
\hline East Transect Auger & FPAH02-09/10-18 & $2 / 25 / 2009$ & $10-18$ & 10 & 22 \\
\hline East Transect Auger & FPAH04-09/3-5.5 & $4 / 7 / 2009$ & $3-5.5$ & 4.6 & n.d. \\
\hline East Transect Auger & FPAH04-09/5-8 & $4 / 7 / 2009$ & $5.5-8$ & 6.1 & n.d. \\
\hline East Transect Auger & FPAH04-09/8-11 & $4 / 7 / 2009$ & 8-11 & 8.4 & n.d. \\
\hline East Transect Auger & FPAH04-09/11-15 & 4/7/2009 & $11-15$ & 10 & n.d. \\
\hline East Transect Auger & FPAH04-09/15-20 & $4 / 7 / 2009$ & $15-20$ & 11 & n.d. \\
\hline East Transect Auger & FPAH04-09/20-25 & $4 / 7 / 2009$ & $20-25$ & 12 & n.d. \\
\hline East Transect Auger & FPAH04-09/25-30 & 4/7/2009 & $25-30$ & 15 & n.d. \\
\hline East Transect Auger & FPAH04-09/30-35 & 4/7/2009 & $30-35$ & 15 & n.d. \\
\hline East Transect Auger & FPAH04-09/35-40 & $4 / 7 / 2009$ & $35-40$ & 17 & n.d. \\
\hline East Transect Auger & HG07-04/0-1 & 11/12/2007 & $0-1$ & 4.1 & 8.8 \\
\hline East Transect Auger & HG07-04/1-5 & $11 / 12 / 2007$ & $1-5$ & 7.7 & 11 \\
\hline East Transect Auger & HG07-04/5-10 & $11 / 12 / 2007$ & $5-10$ & n.d. & 14 \\
\hline
\end{tabular}


Table 7. Moisture content of sediment collected by augering at Franklin Lake playa._-Continued

[Sample site, name of sampling site; Sample ID, unique sample identifier; Gravimetric water content, determined by weight loss on heating at $105^{\circ} \mathrm{C}$; Adsorbed water, the amount of water adsorbed by the dried sample in an atmosphere of $100 \%$ humidity; n.d., not determined; cm, centimeters; wt.\%, weight percent]

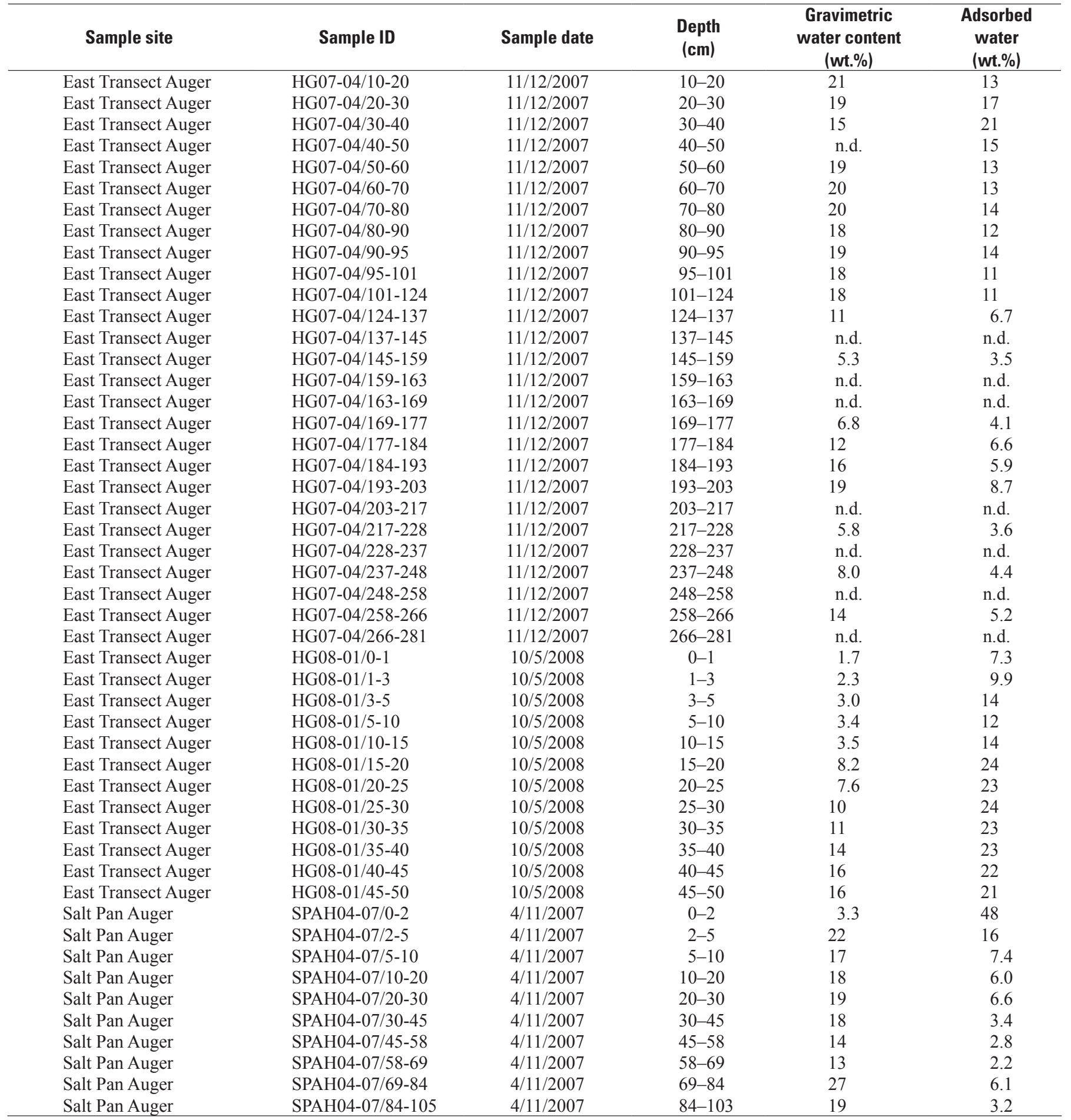


Table 8. Carbonate percent of selected grab samples.

[Sample site, name of sampling site; Sample ID, unique sample identifier; \%, percent]

\begin{tabular}{llc}
\hline \multicolumn{1}{c}{ Sample site } & Sample ID & Carbonate \% \\
\hline Amargosa River Crossing & JY05 35a & 9.62 \\
Amargosa River Crossing & JY05 35b & 9.76 \\
Amargosa River Crossing & JY05 35c & 14.20 \\
Amargosa River East of Death Valley Junction & JY05 37a & 21.38 \\
Amargosa River East of Death Valley Junction & JY05 37b & 20.98 \\
Amargosa River East of Death Valley Junction & JY05 37c & 15.17 \\
Amargosa River East of Death Valley Junction & JY05 38a & 7.69 \\
Amargosa River East of Death Valley Junction & JY05 38b & 7.27 \\
Amargosa River East of Death Valley Junction & JY05 38c & 8.23 \\
Ash Meadows & JY05-29 & 37.09 \\
Clay Dune & JY05-25 & 16.36 \\
Coppice & JY04-15a & 12.58 \\
Coppice & JY04-15b & 11.72 \\
Coppice & JY04-15c & 13.26 \\
Coppice & JY04-16a & 11.71 \\
Coppice & JY04-16b & 14.14 \\
Coppice & JY04-16c & 14.97 \\
Coppice & JY05-17 & 13.55 \\
Discovery & JY-03-01 & 21.64 \\
Discovery & JY-03-01a & 21.41 \\
Discovery & JY-03-01b & 20.41 \\
Discovery & JY05-15 & 21.25 \\
Discovery & JY05-16 & 26.01 \\
East Transect & JY05-21a & 29.56 \\
East Transect & JY05-21b & 19.86 \\
Ojo del Caballo & JY05-30 \\
West Transect & JY05-19 & 32.96 \\
& & 15.18 \\
\hline
\end{tabular}


Table 9A. Particle-size data for the East Transect auger hole. Each value represents the percent of the total sample present in each respective size bin. Size bins based on the Wentworth scale (Wentworth, 1922).

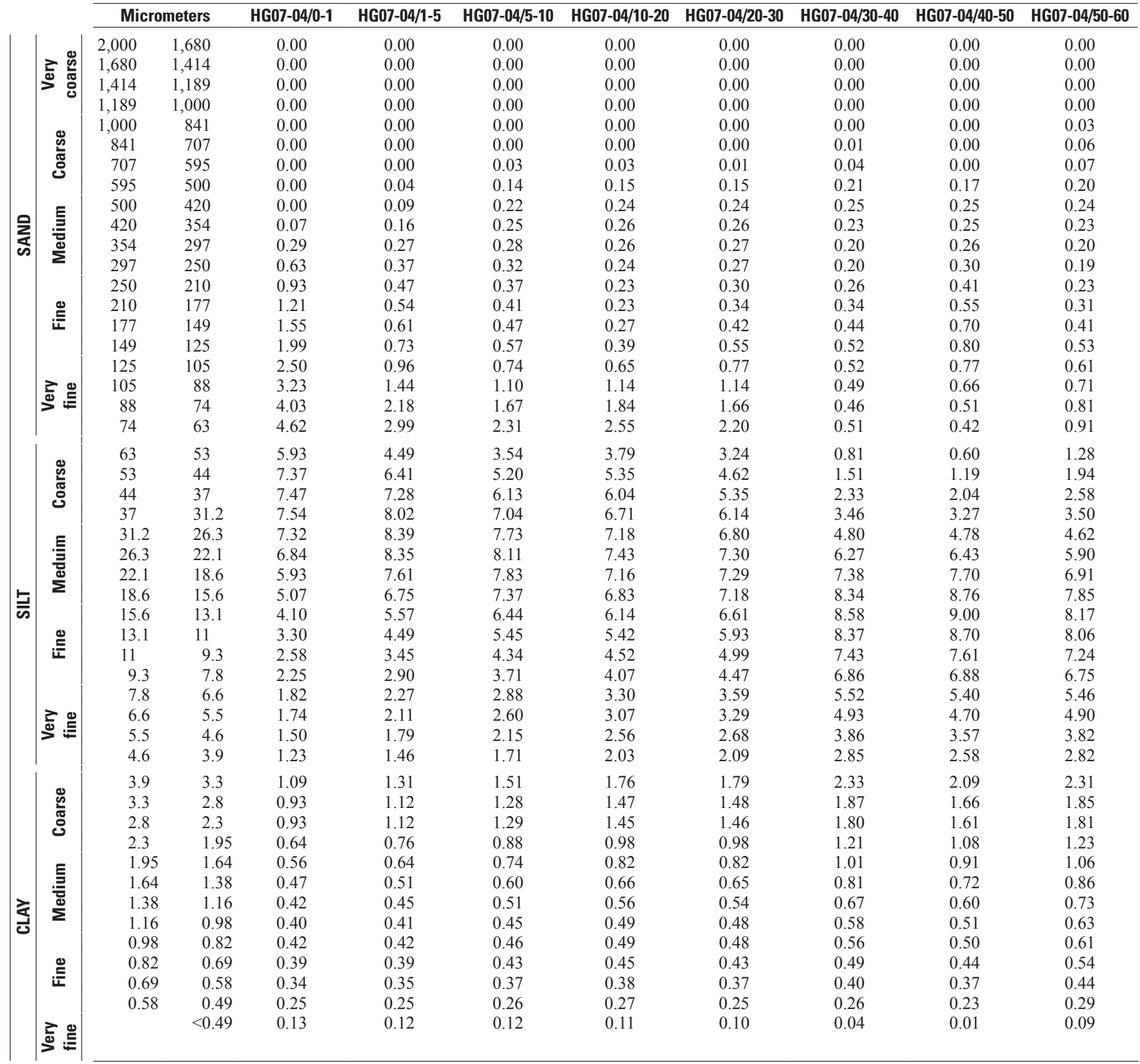


Table 9A. Particle-size data for the East Transect auger hole. Each value represents the percent of the total sample present in each respective size bin. Size bins based on the Wentworth scale (Wentworth, 1922).- Continued

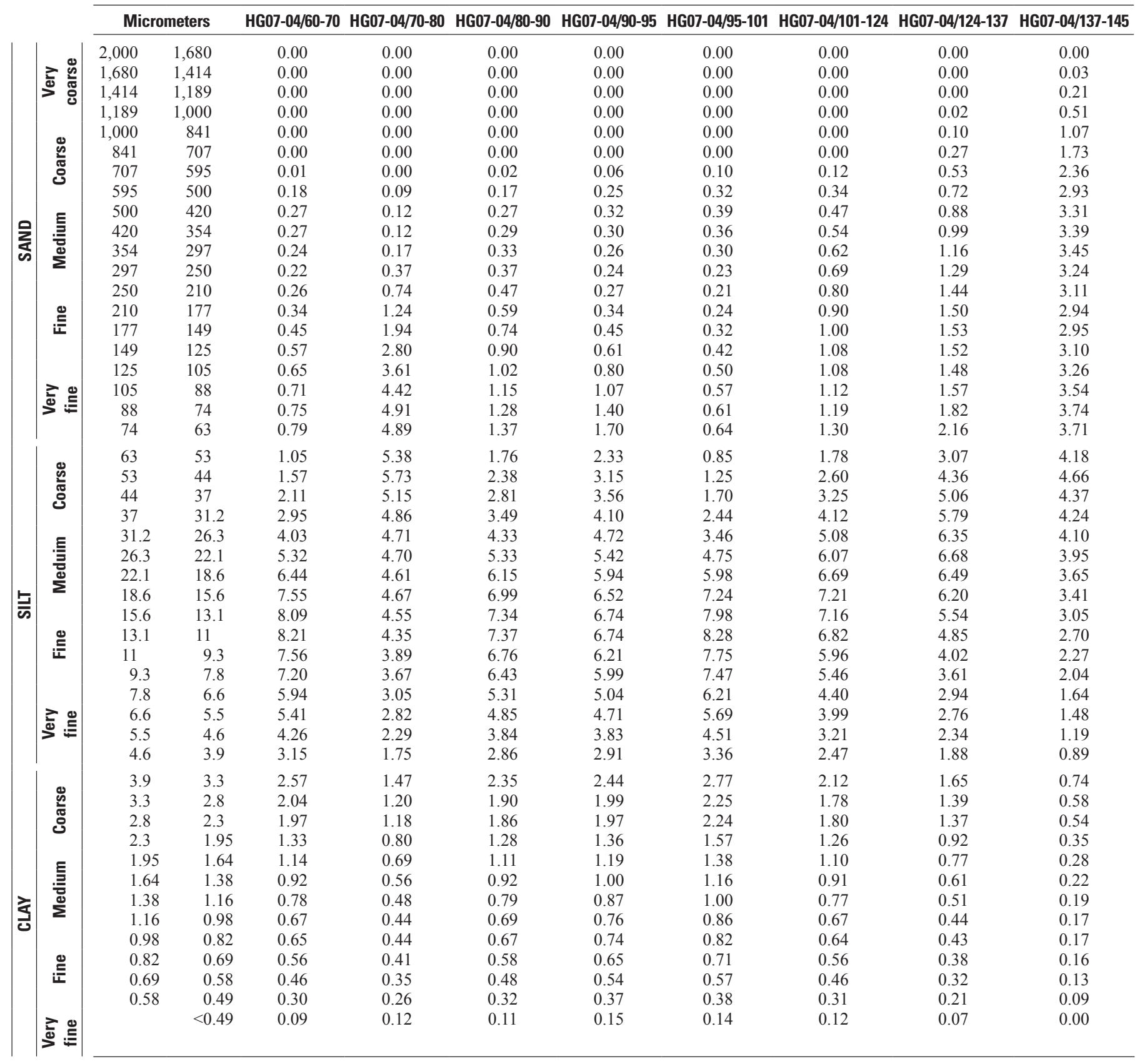


Tables

Table 9A. Particle-size data for the East Transect auger hole. Each value represents the percent of the total sample present in each respective size bin. Size bins based on the Wentworth scale (Wentworth, 1922).-Continued

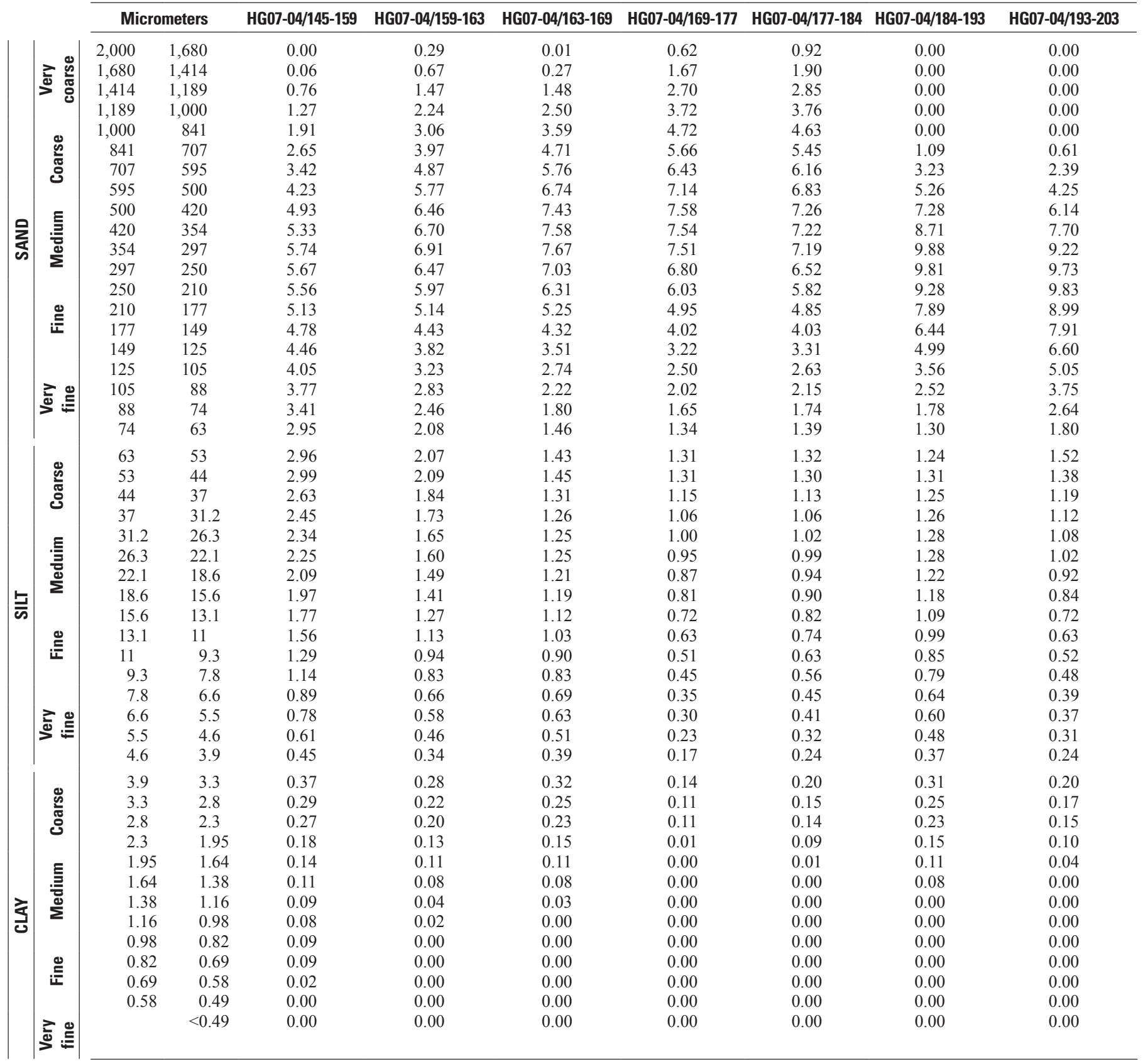


Table 9A. Particle-size data for the East Transect auger hole. Each value represents the percent of the total sample present in each respective size bin. Size bins based on the Wentworth scale (Wentworth, 1922).-Continued

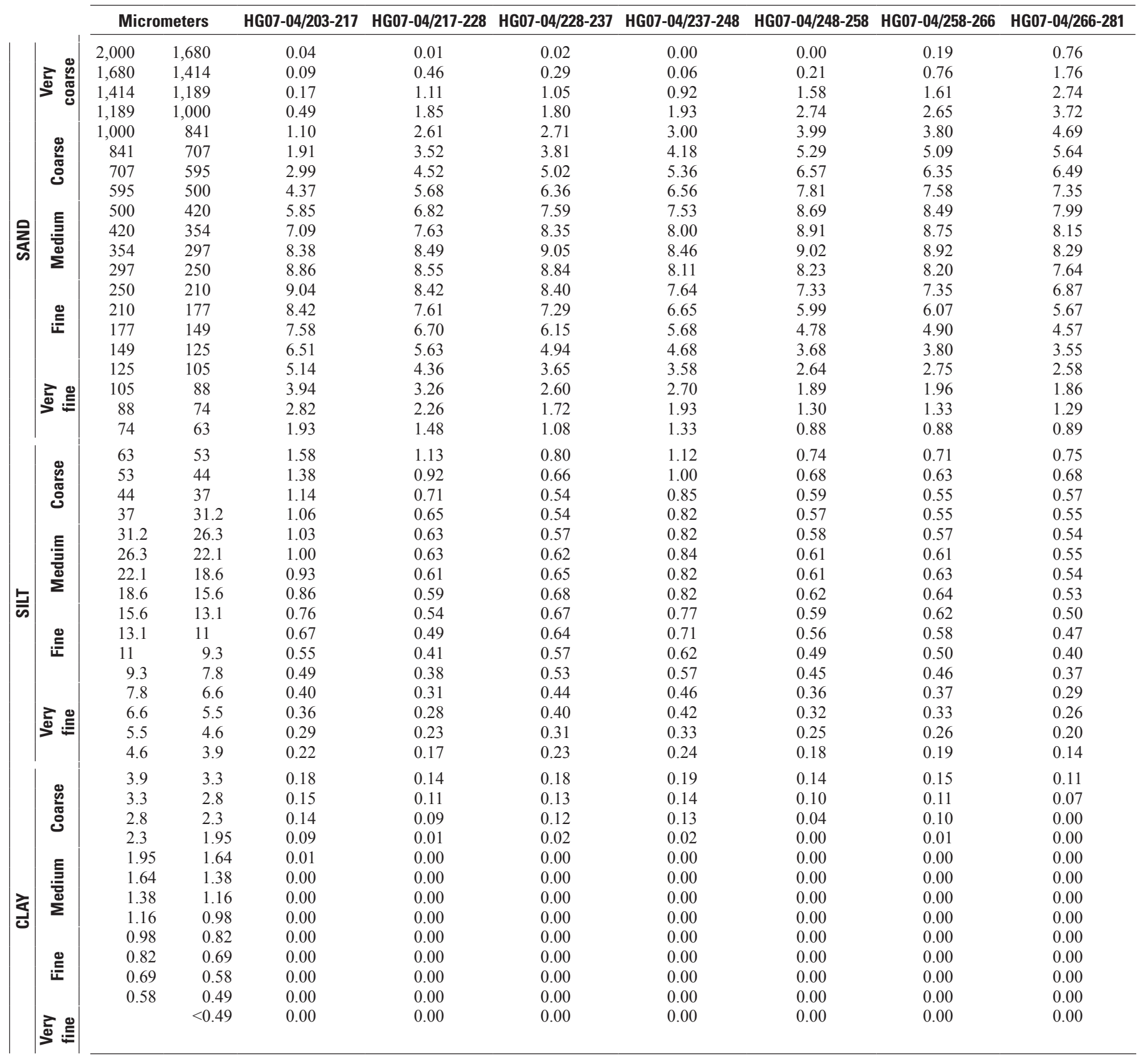


Table 9B. Particle-size data for the Coppice dune site auger hole. Each value represents the percent of the total sample present in each respective size bin. Size bins based on the Wentworth scale (Wentworth, 1922).

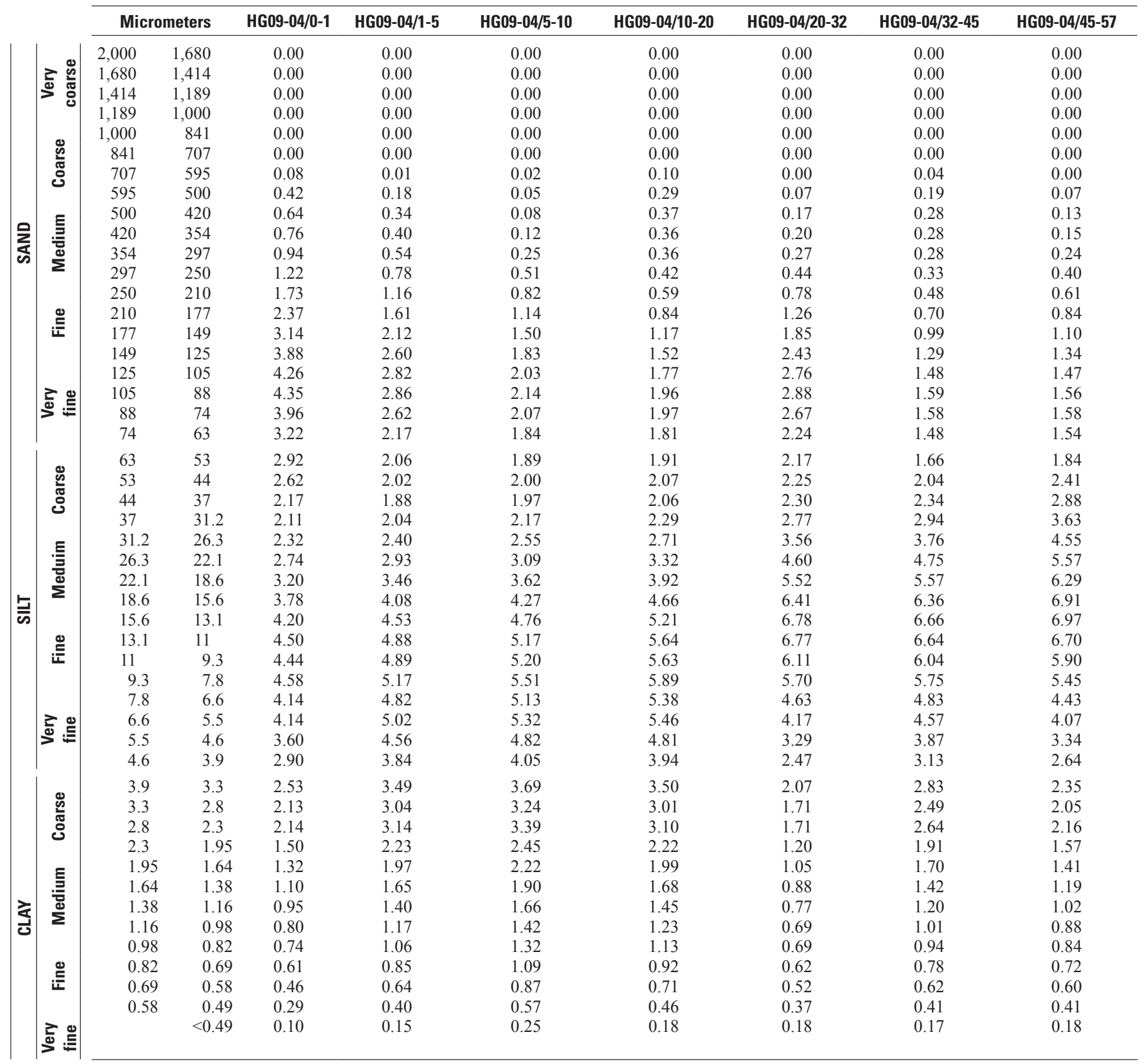


Table 9B. Particle-size data for the Coppice dune site auger hole. Each value represents the percent of the total sample present in each respective size bin. Size bins based on the Wentworth scale (Wentworth, 1922).-_ Continued

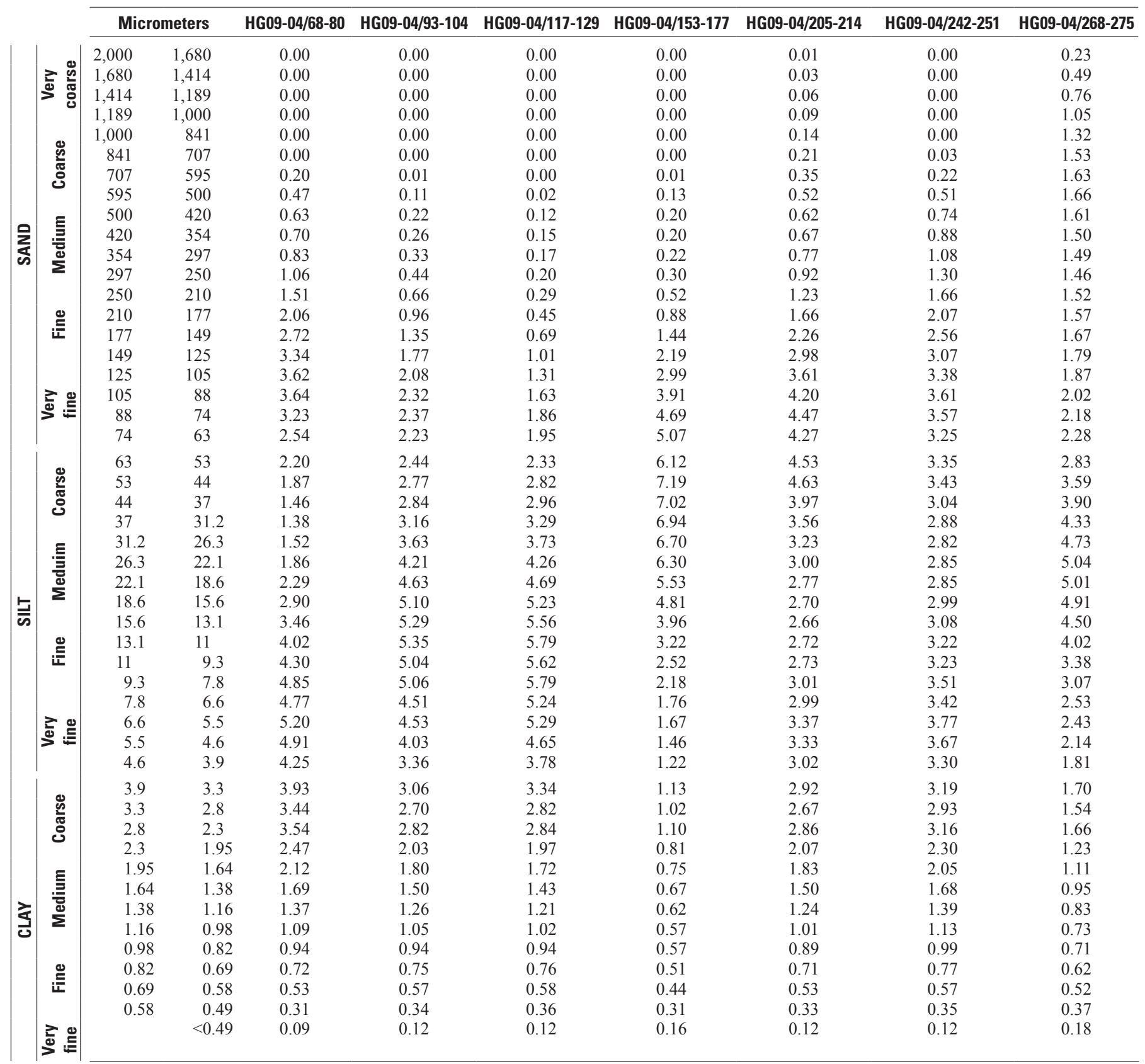


Table 9B. Particle-size data for the Coppice dune site auger hole. Each value represents the percent of the total sample present in each respective size bin. Size bins based on the Wentworth scale (Wentworth, 1922).-Continued

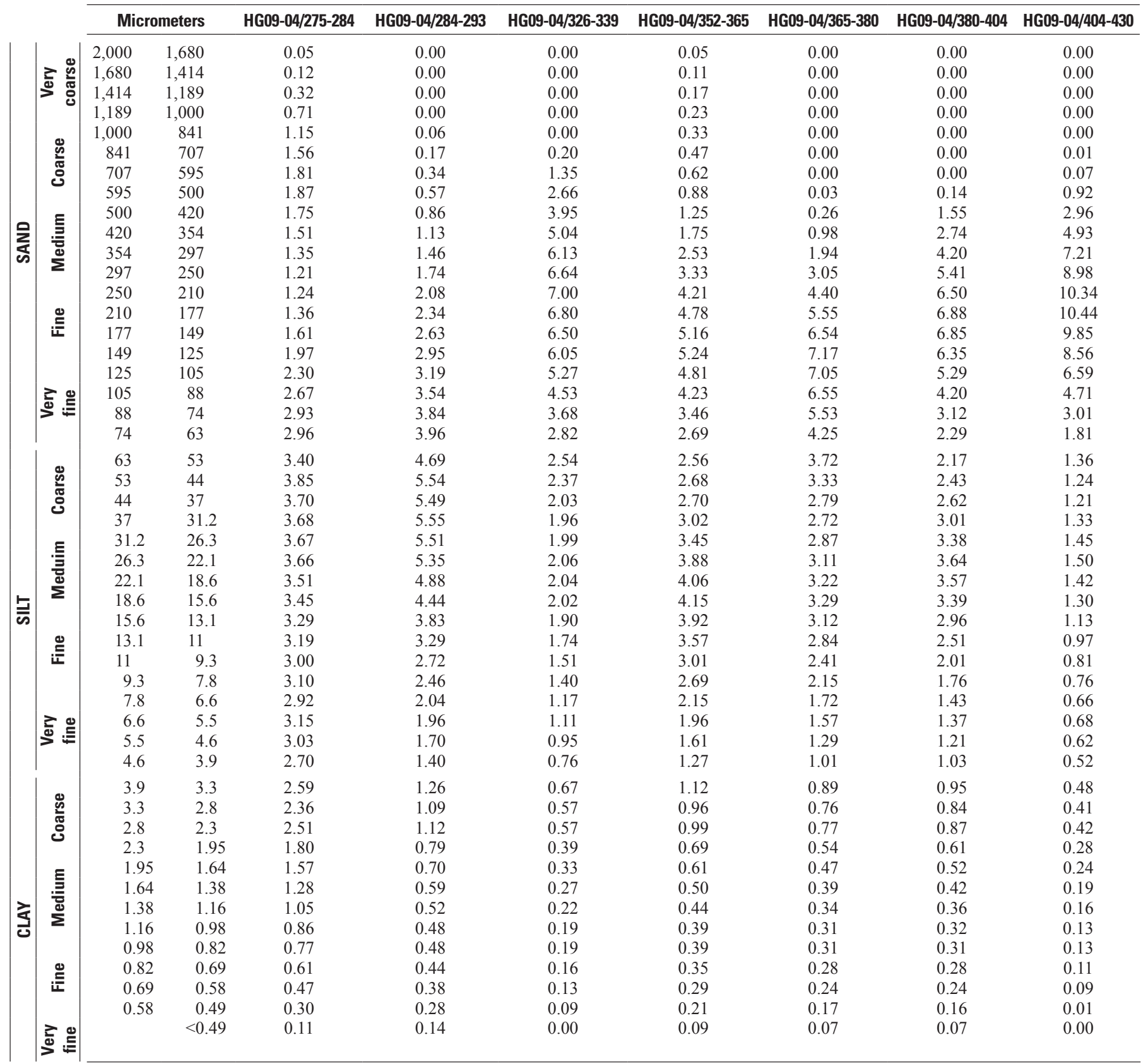


Table 9C. Particle-size data for Franklin Lake playa and Ash meadows grab samples. Each value represents the percent of the total sample present in each respective size bin. Size bins based on the Wentworth scale (Wentworth, 1922).

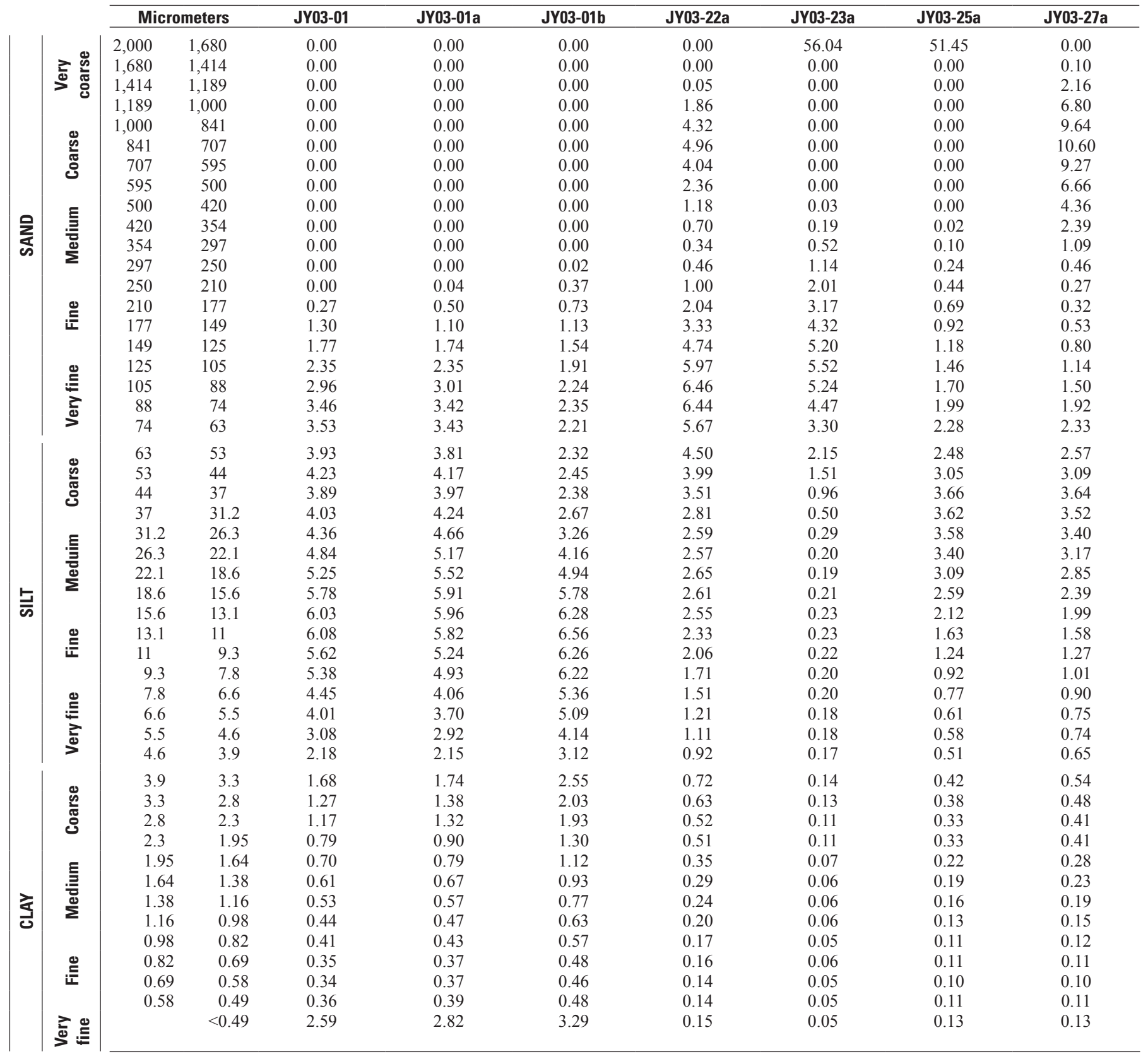


Tables

Table 9C. Particle-size data for Franklin Lake playa and Ash meadows grab samples. Each value represents the percent of the total sample present in each respective size bin. Size bins based on the Wentworth scale (Wentworth, 1922).—Continued

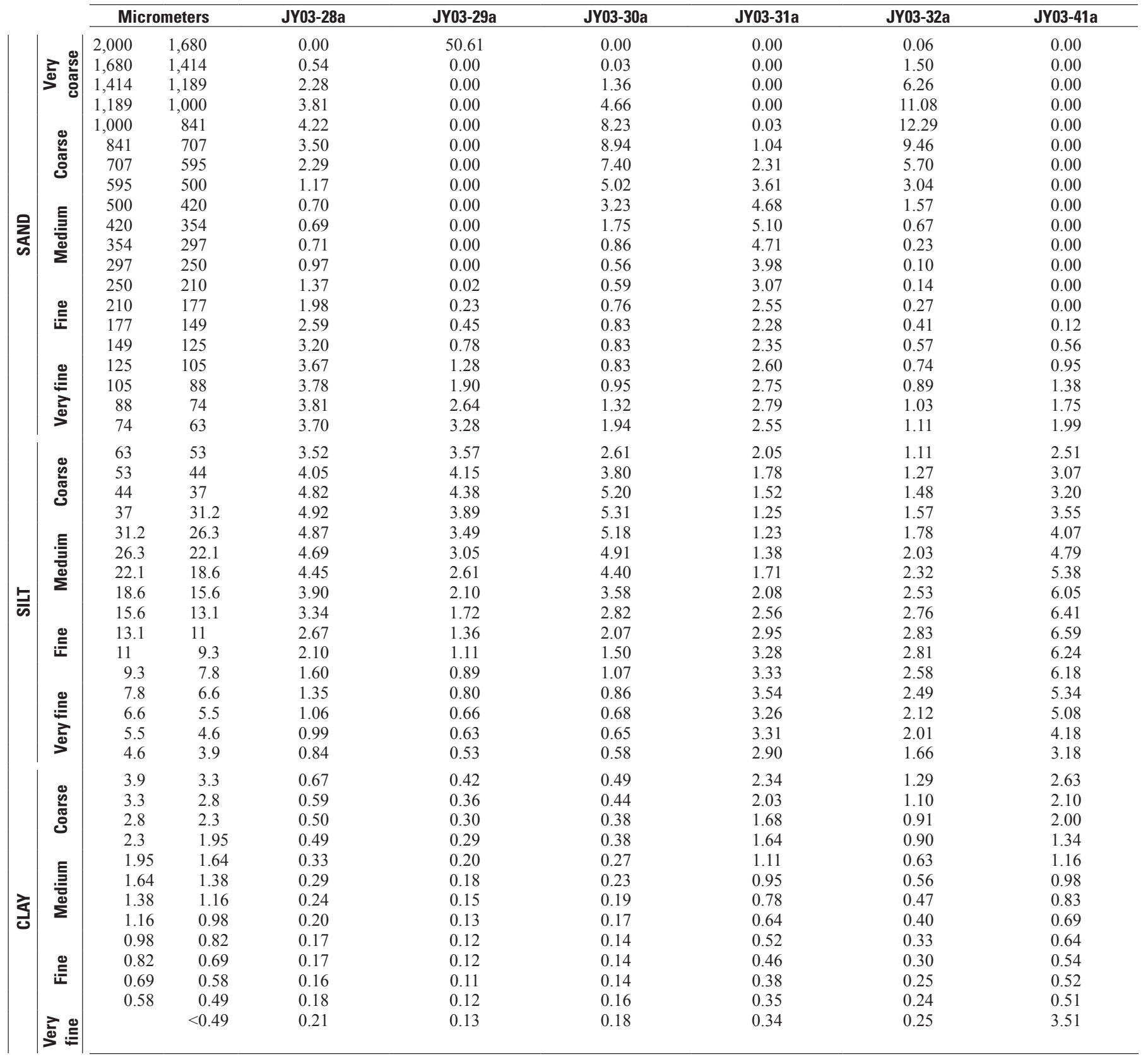


Table 9C. Particle-size data for Franklin Lake playa and Ash meadows grab samples. Each value represents the percent of the total sample present in each respective size bin. Size bins based on the Wentworth scale (Wentworth, 1922).-Continued

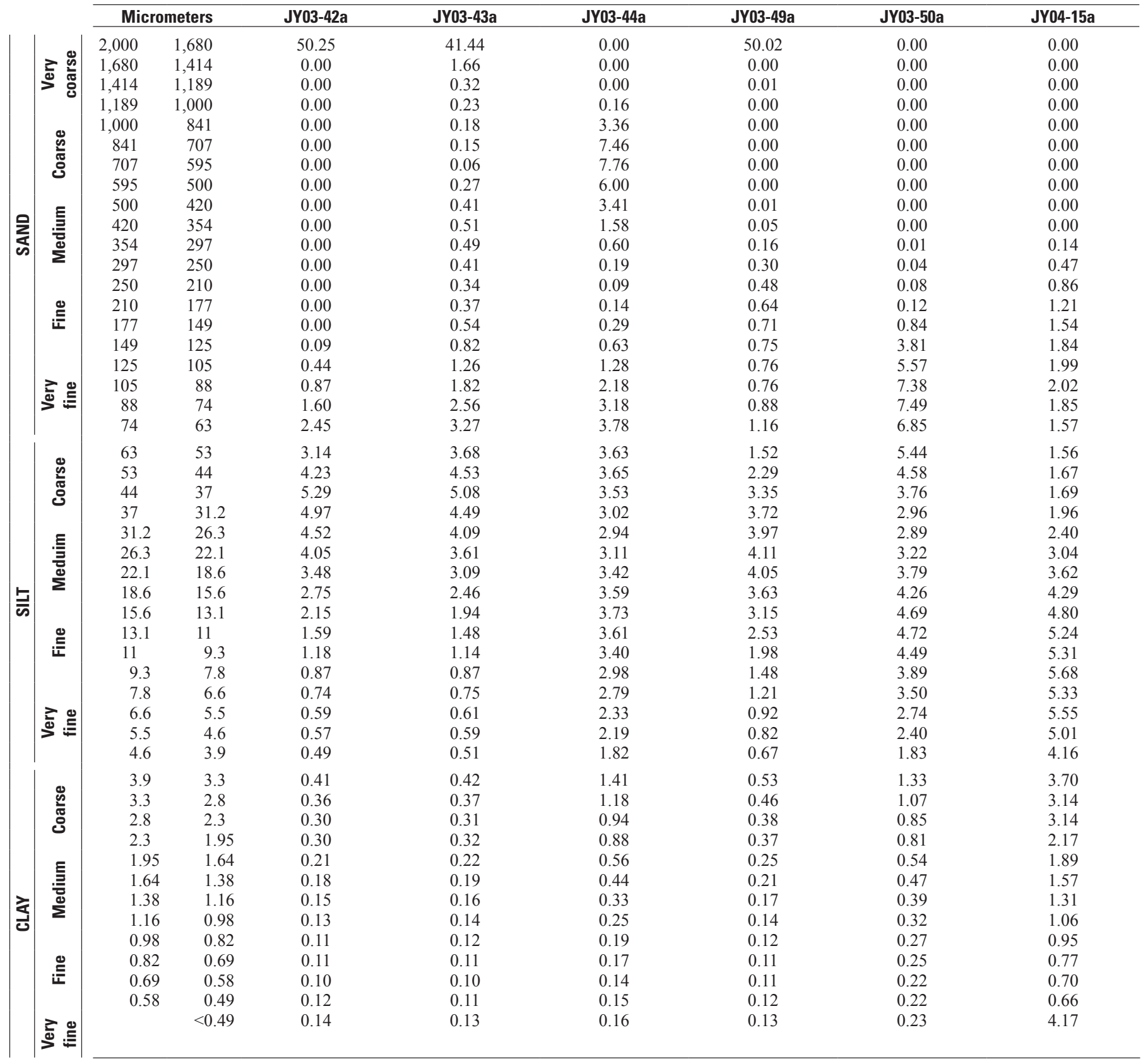


Tables

Table 9C. Particle-size data for Franklin Lake playa and Ash meadows grab samples. Each value represents the percent of the total sample present in each respective size bin. Size bins based on the Wentworth scale (Wentworth, 1922).-Continued

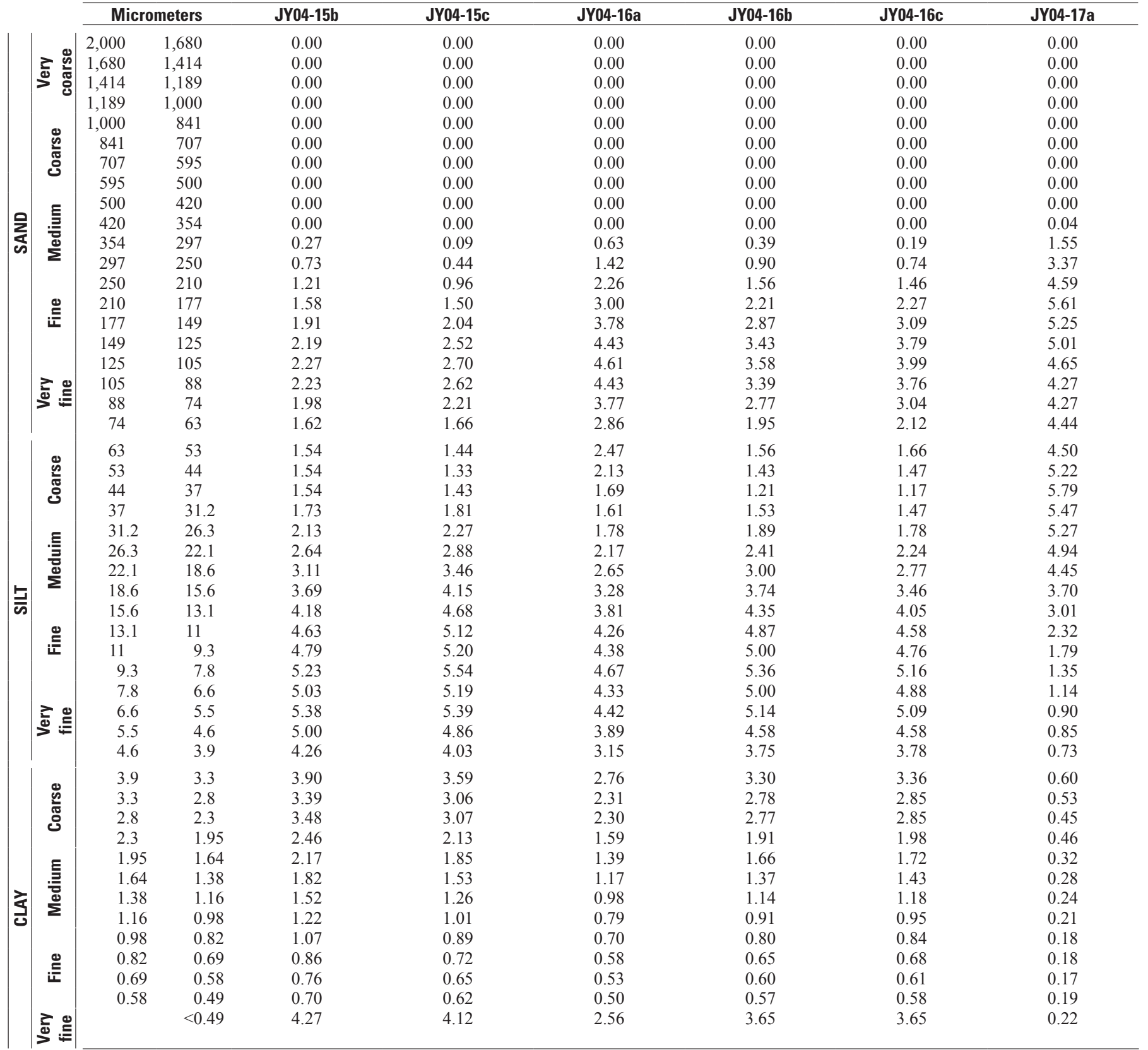


Table 9C. Particle-size data for Franklin Lake playa and Ash meadows grab samples. Each value represents the percent of the total sample present in each respective size bin. Size bins based on the Wentworth scale (Wentworth, 1922).

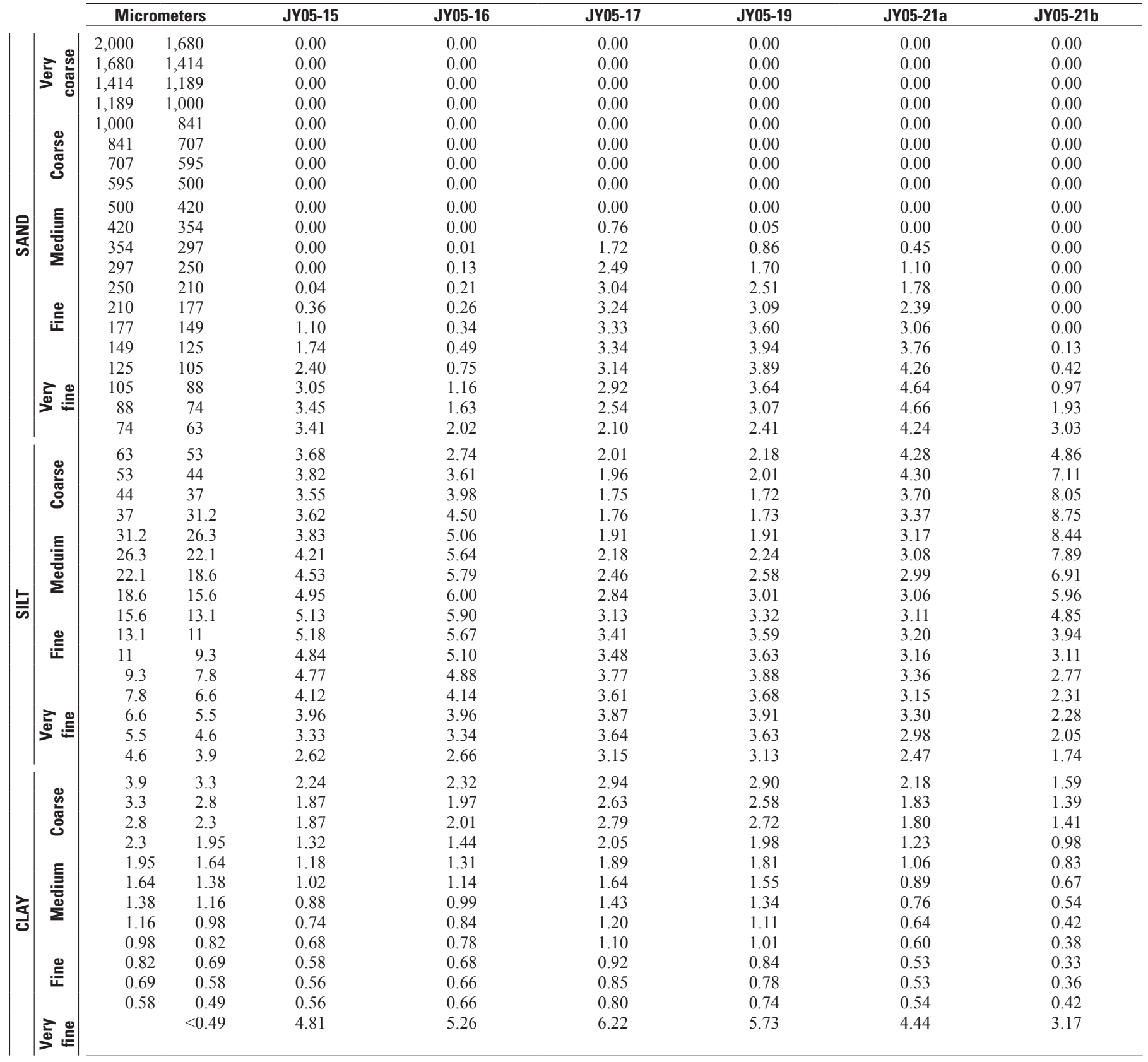


Tables

Table 9C. Particle-size data for Franklin Lake playa and Ash meadows grab samples. Each value represents the percent of the total sample present in each respective size bin. Size bins based on the Wentworth scale (Wentworth, 1922).—Continued

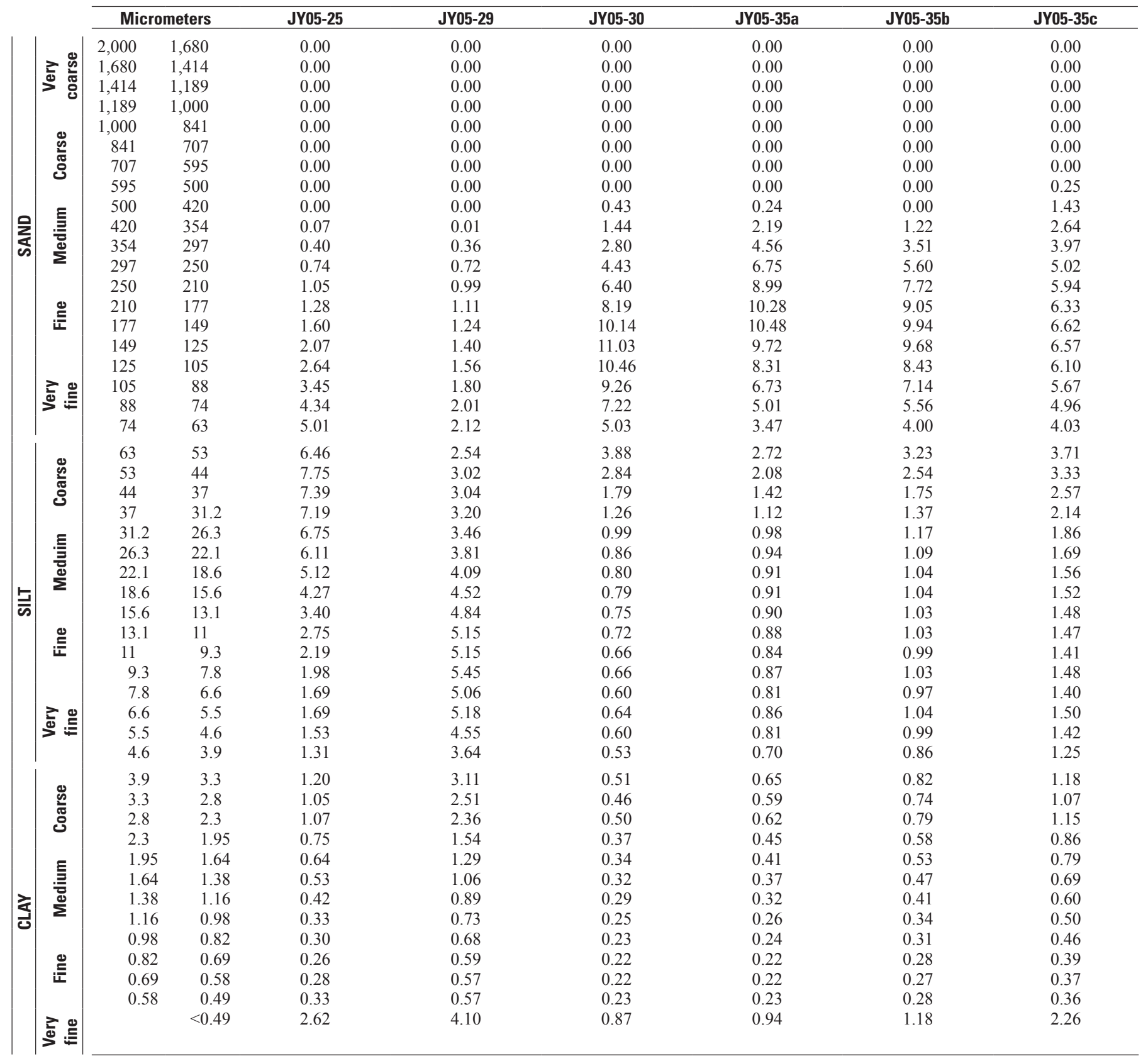


Table 9C. Particle-size data for Franklin Lake playa and Ash meadows grab samples. Each value represents the percent of the total sample present in each respective size bin. Size bins based on the Wentworth scale (Wentworth, 1922).-Continued

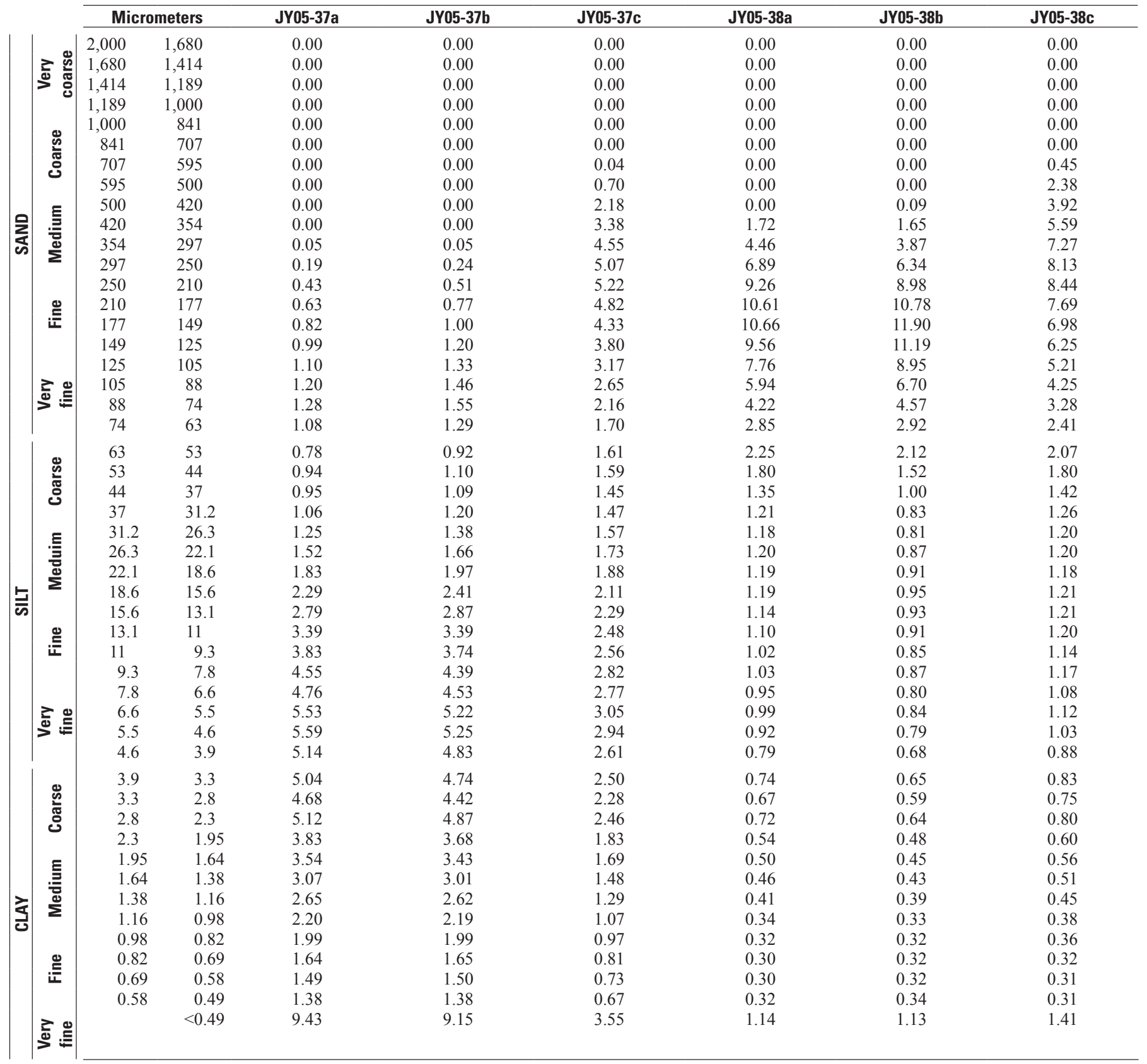


Table 10. Bulk density data of Franklin Lake playa grab samples.

[Sample site, name of sampling site; Sample ID, unique sample identifier; Ring size, height of sampling ring; $\mathrm{cm}$, centimeters; $\mathrm{g} / \mathrm{cm}^{3}, \mathrm{grams}$ per cubic centimeter]

\begin{tabular}{|c|c|c|c|}
\hline Sample site & Sample ID & Ring size & $\begin{array}{c}\text { Bulk density } \\
\left(\mathrm{g} / \mathrm{cm}^{3}\right)\end{array}$ \\
\hline Amargosa River Crossing & JY05-35a & $1 \mathrm{~cm}$ & 1.87 \\
\hline Amargosa River Crossing & JY05-35b & $3 \mathrm{~cm}$ & 1.32 \\
\hline Amargosa River Crossing & JY05-35c & $3 \mathrm{~cm}$ & 1.48 \\
\hline Amargosa River East of Death Valley Junction & JY05-37a & $1 \mathrm{~cm}$ & 1.33 \\
\hline Amargosa River East of Death Valley Junction & JY05-37b & $3 \mathrm{~cm}$ & 1.42 \\
\hline Amargosa River East of Death Valley Junction & JY05-37c & $3 \mathrm{~cm}$ & 1.40 \\
\hline Amargosa River East of Death Valley Junction & JY05-38a & $1 \mathrm{~cm}$ & 1.17 \\
\hline Amargosa River East of Death Valley Junction & JY05-38b & $3 \mathrm{~cm}$ & 1.33 \\
\hline Amargosa River East of Death Valley Junction & JY05-38c & $3 \mathrm{~cm}$ & 1.62 \\
\hline Clay Dune & JY05-24a & $1 \mathrm{~cm}$ & 1.18 \\
\hline Clay Dune & $J Y 05-24 b$ & $3 \mathrm{~cm}$ & 0.79 \\
\hline Clay Dune & JY05-24c & $3 \mathrm{~cm}$ & 0.68 \\
\hline Coppice & JY05-14a & $1 \mathrm{~cm}$ & 1.38 \\
\hline Coppice & JY05-14b & $3 \mathrm{~cm}$ & 1.23 \\
\hline Coppice & JY05-14c & $3 \mathrm{~cm}$ & 1.10 \\
\hline Discovery & JY05-12a & $1 \mathrm{~cm}$ & 0.83 \\
\hline Discovery & JY05-12b & $3 \mathrm{~cm}$ & 1.48 \\
\hline Discovery & JY05-12c & $3 \mathrm{~cm}$ & 1.07 \\
\hline Discovery & JY05-13a & $1 \mathrm{~cm}$ & 1.08 \\
\hline Discovery & JY05-13b & $3 \mathrm{~cm}$ & 1.26 \\
\hline Discovery & JY05-13c & $3 \mathrm{~cm}$ & 1.23 \\
\hline East Transect & JY05-20a & $1 \mathrm{~cm}$ & 1.13 \\
\hline East Transect & JY05-20b & $3 \mathrm{~cm}$ & 0.99 \\
\hline East Transect & JY05-20c & $3 \mathrm{~cm}$ & 0.73 \\
\hline Franklin Lake Playa/Carson Slough & JY05-27 & $1 \mathrm{~cm}$ & 1.21 \\
\hline Franklin Lake Playa/Carson Slough & JY05-28 & $1 \mathrm{~cm}$ & 1.60 \\
\hline Salt Pan & JY05-22a & $1 \mathrm{~cm}$ & 0.71 \\
\hline Salt Pan & JY05-22b & $3 \mathrm{~cm}$ & 0.63 \\
\hline Salt Pan & $J Y 05-22 c$ & $3 \mathrm{~cm}$ & 0.73 \\
\hline West Transect & JY05-18a & $1 \mathrm{~cm}$ & 1.40 \\
\hline West Transect & JY05-18b & $3 \mathrm{~cm}$ & 1.37 \\
\hline West Transect & JY05-18c & $3 \mathrm{~cm}$ & 1.23 \\
\hline
\end{tabular}


Table 11A. Geochemical data determined on selected bulk East transect auger hole samples. Method of determination (ICP-MS, inductively coupled plasma-mass spectrometry; ICP-OES, inductively coupled plasma-optical emission spectrometry) is listed above each element.

[Sample site, name of sampling site; Sample ID, unique sample identifier; ppm, parts per million; wt.\%, weight percent]

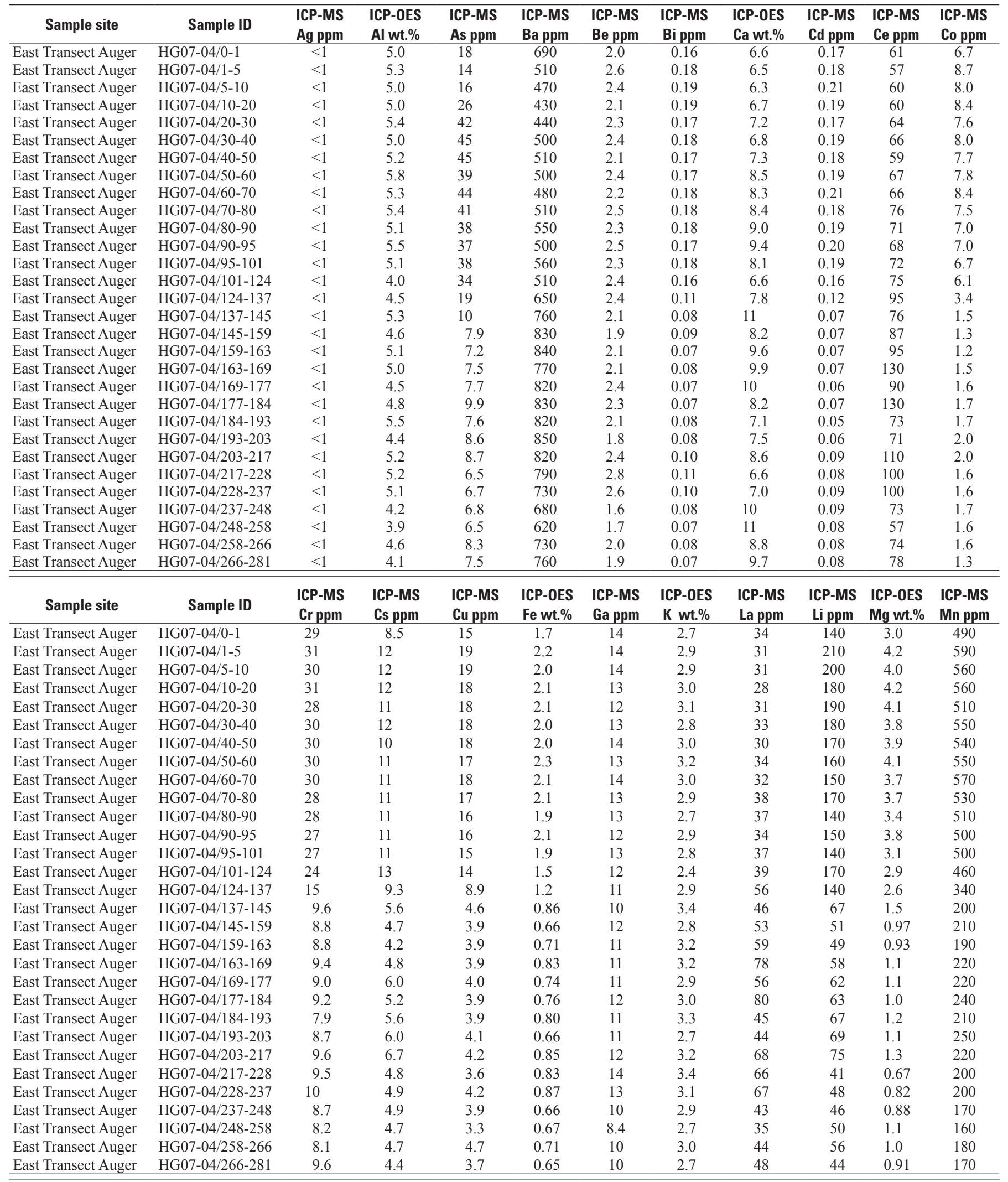


Table 11A. Geochemical data determined on selected bulk East transect auger hole samples. Method of determination (ICP-MS, inductively coupled plasma-mass spectrometry; ICP-OES, inductively coupled plasma-optical emission spectrometry) is listed above each element.-Continued

[Sample site, name of sampling site; Sample ID, unique sample identifier; ppm, parts per million; wt.\%, weight percent]

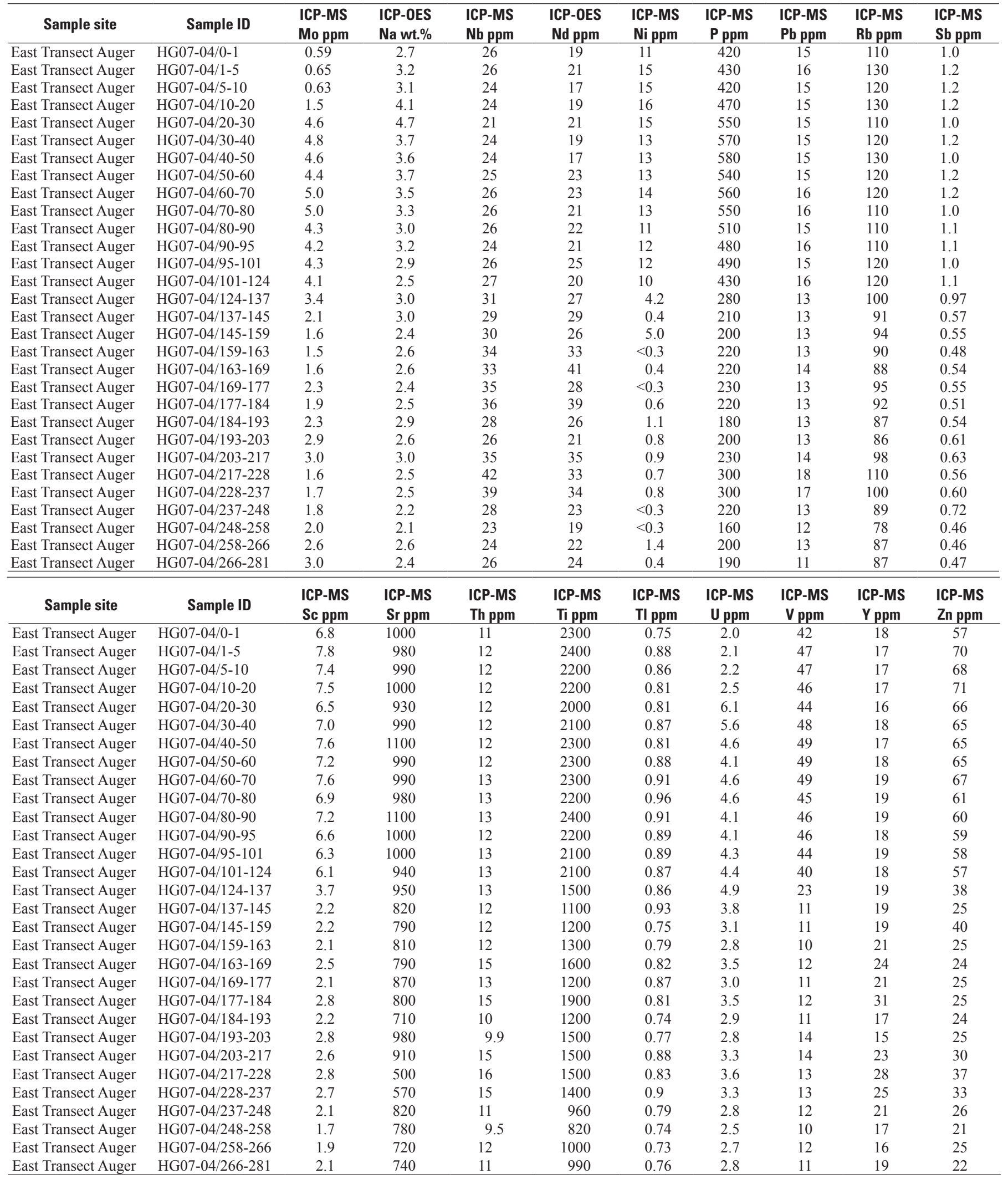


Table 11B. Geochemical data determined on selected bulk grab samples. Method of determination (ICP-MS, inductively coupled plasmamass spectrometry; ICP-OES, inductively coupled plasma-optical emission spectrometry) is listed above each element.

[Sample site, name of sampling site; Sample ID, unique sample identifier; ppm, parts per million; wt.\%, weight percent]

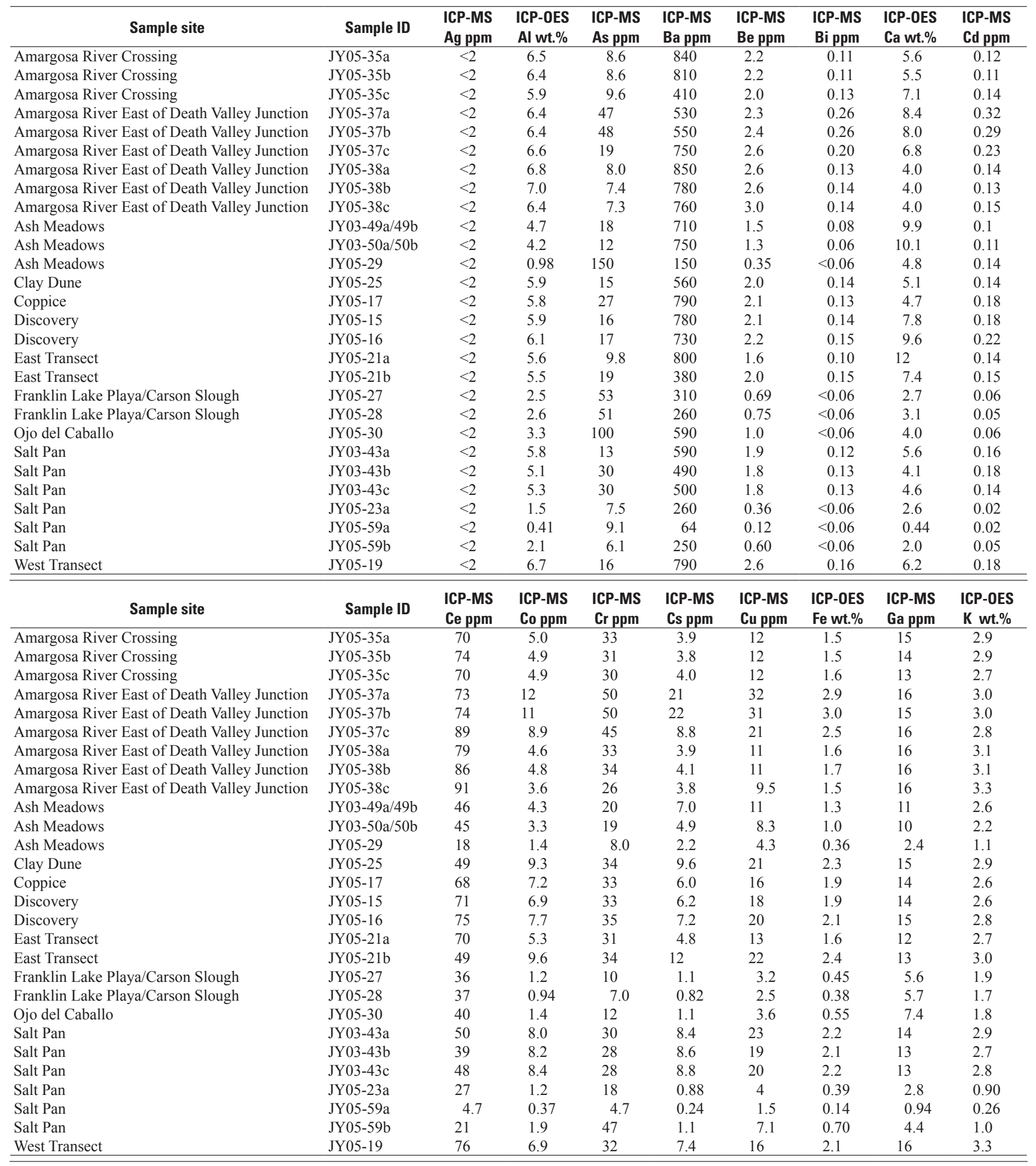


Table 11B. Geochemical data determined on selected bulk grab samples. Method of determination (ICP-MS, inductively coupled plasmamass spectrometry; ICP-OES, inductively coupled plasma-optical emission spectrometry) is listed above each element.—Continued

[Sample site, name of sampling site; Sample ID, unique sample identifier; ppm, parts per million; wt.\%, weight percent]

\begin{tabular}{|c|c|c|c|c|c|c|c|c|}
\hline Sample site & Sample ID & $\begin{array}{l}\text { ICP-MS } \\
\text { La ppm }\end{array}$ & $\begin{array}{l}\text { ICP-MS } \\
\text { Li ppm }\end{array}$ & $\begin{array}{l}\text { ICP-0ES } \\
\text { Mg wt.\% }\end{array}$ & $\begin{array}{l}\text { ICP-MS } \\
\text { Mn ppm }\end{array}$ & $\begin{array}{l}\text { ICP-MS } \\
\text { Mo ppm }\end{array}$ & $\begin{array}{l}\text { ICP-0ES } \\
\text { Na wt.\% }\end{array}$ & $\begin{array}{l}\text { ICP-MS } \\
\text { Nb ppm }\end{array}$ \\
\hline Amargosa River Crossing & JY05-35b & 42 & 55 & 1.9 & 470 & 1.3 & 1.8 & 13 \\
\hline Amargosa River Crossing & JY05-35c & 39 & 57 & 2.0 & 460 & 1.2 & 1.5 & 17 \\
\hline Amargosa River East of Death Valley Junction & JY05-37b & 41 & 250 & 2.8 & 640 & 8.4 & 1.1 & 20 \\
\hline Amargosa River East of Death Valley Junction & JY05-37c & 49 & 100 & 1.8 & 680 & 2.9 & 1.7 & 23 \\
\hline Amargosa River East of Death Valley Junction & JY05-38a & 47 & 50 & 1.1 & 430 & 1.4 & 2.3 & 17 \\
\hline Ash Meadows & $J Y 03-49 a / 49 b$ & 26 & 130 & 3.1 & 340 & 1.4 & 3.1 & 9.4 \\
\hline Ash Meadows & $J Y 03-50 a / 50 b$ & 24 & 85 & 2.1 & 290 & 1.6 & 4.8 & 6.8 \\
\hline Ash Meadows & JY05-29 & 9.7 & 29 & 0.68 & 91 & 95 & 23 & 2.8 \\
\hline Clay Dune & JY05-25 & 29 & 280 & 3.8 & 630 & 0.57 & 3.3 & 15 \\
\hline Coppice & JY05-17 & 40 & 72 & 1.4 & 540 & 7.6 & 6.8 & 16 \\
\hline Discovery & JY05-15 & 39 & 82 & 2.0 & 500 & 2.8 & 4.6 & 16 \\
\hline Ojo del Caballo & JY05-30 & 23 & 19 & 0.45 & 140 & 15 & 15 & 8.2 \\
\hline Salt Pan & JY03-43a & 29 & 260 & 3.2 & 560 & 0.78 & 3.5 & 12 \\
\hline Salt Pan & JY03-43b & 23 & 490 & 3.2 & 550 & 4.6 & 5.6 & 9.8 \\
\hline Salt Pan & JY03-43c & 28 & 490 & 3.2 & 570 & 3.2 & 4.6 & 9.9 \\
\hline Salt Pan & JY05-23a & 12 & 22 & 0.31 & 110 & 1.2 & 19 & 2.5 \\
\hline Salt Pan & JY05-59a & 2.6 & 18 & 0.08 & 27 & 3.4 & 27 & 1.8 \\
\hline Salt Pan & JY05-59b & 12 & 33 & 0.37 & 120 & 2.0 & 18 & 5.2 \\
\hline West Transect & JY05-19 & 41 & 110 & 2.3 & 580 & 1.8 & 2.5 & 21 \\
\hline Sample site & Sample ID & $\begin{array}{l}\text { ICP-OES } \\
\text { Nd ppm }\end{array}$ & $\begin{array}{l}\text { ICP-MS } \\
\text { Ni ppm }\end{array}$ & $\begin{array}{l}\text { ICP-MS } \\
\text { P ppm }\end{array}$ & $\begin{array}{l}\text { ICP-MS } \\
\text { Pb ppm }\end{array}$ & $\begin{array}{l}\text { ICP-MS } \\
\text { Rb ppm }\end{array}$ & $\begin{array}{l}\text { ICP-MS } \\
\text { Sb ppm }\end{array}$ & $\begin{array}{l}\text { ICP-MS } \\
\text { Sc ppm }\end{array}$ \\
\hline Amargosa River Crossing & JY05-35a & 26 & 9.6 & 420 & 19 & 100 & 0.85 & 5.4 \\
\hline Amargosa River Crossing & JY05-35b & 27 & 9.5 & 410 & 18 & 99 & 0.82 & 5.5 \\
\hline Ash Meadows & JY05-29 & 6.1 & 3.1 & 590 & 4.1 & 21 & 0.47 & 1.6 \\
\hline Clay Dune & JY05-25 & 21 & 17 & 370 & 17 & 130 & 1.2 & 7.4 \\
\hline Coppice & JY05-17 & 29 & 17 & 700 & 20 & 110 & 1.1 & 6.6 \\
\hline Discovery & JY05-15 & 29 & 14 & 630 & 19 & 110 & 1 & 7.1 \\
\hline Discovery & JY05-16 & 30 & 16 & 670 & 21 & 120 & 1.1 & 7.7 \\
\hline East Transect & JY05-21a & 29 & 10 & 430 & 18 & 94 & 0.7 & 6.2 \\
\hline East Transect & JY05-21b & 21 & 18 & 440 & 15 & 130 & 1.2 & 8.0 \\
\hline Franklin Lake Playa/Carson Slough & JY05-27 & 12 & 2.2 & 180 & 6.4 & 45 & 0.55 & 1.8 \\
\hline Franklin Lake Playa/Carson Slough & JY05-28 & 13 & 1.6 & 160 & 6.1 & 40 & 0.41 & 1.5 \\
\hline Ojo del Caballo & JY05-30 & 16 & 2.8 & 540 & 8.6 & 49 & 0.4 & 2.3 \\
\hline Salt Pan & JY03-43a & 21 & 15 & 380 & 16 & 110 & 1.1 & 6.8 \\
\hline Salt Pan & JY03-43b & 16 & 15 & 380 & 14 & 110 & 0.97 & 7.0 \\
\hline Salt Pan & JY03-43c & 19 & 16 & 390 & 15 & 110 & 0.99 & 7.3 \\
\hline Salt Pan & JY05-23a & 10 & 2.5 & 230 & 5.3 & 25 & 0.22 & 1.6 \\
\hline Salt Pan & JY05-59a & $<4$ & 0.8 & 55 & 1.2 & 6.7 & 0.1 & 0.5 \\
\hline Salt Pan & JY05-59b & 10 & 9.3 & 150 & 5.3 & 30 & 0.28 & 2.0 \\
\hline West Transect & JY05-19 & 30 & 14 & 600 & 21 & 130 & 1.2 & 7.3 \\
\hline
\end{tabular}


Table 11B. Geochemical data determined on selected bulk grab samples. Method of determination (ICP-MS, inductively coupled plasmamass spectrometry; ICP-OES, inductively coupled plasma-optical emission spectrometry) is listed above each element.-Continued

[Sample site, name of sampling site; Sample ID, unique sample identifier; ppm, parts per million; wt.\%, weight percent]

\begin{tabular}{|c|c|c|c|c|c|c|c|c|c|}
\hline Sample site & Sample ID & $\begin{array}{l}\text { ICP-MS } \\
\text { Sr ppm }\end{array}$ & $\begin{array}{l}\text { ICP-MS } \\
\text { Th ppm }\end{array}$ & $\begin{array}{l}\text { ICP-MS } \\
\text { Ti ppm }\end{array}$ & $\begin{array}{c}\text { ICP-MS } \\
\text { TI ppm }\end{array}$ & $\begin{array}{c}\text { ICP-MS } \\
\text { U ppm }\end{array}$ & $\begin{array}{c}\text { ICP-MS } \\
\text { V ppm }\end{array}$ & $\begin{array}{c}\text { ICP-MS } \\
\text { Y ppm }\end{array}$ & $\begin{array}{l}\text { ICP-MS } \\
\text { Zn ppm }\end{array}$ \\
\hline Amargosa River Crossing & JY05-35b & 450 & 12 & 1,900 & 0.60 & 2.4 & 35 & 18 & 46 \\
\hline Amargosa River Crossing & JY05-35c & 440 & 11 & 1,900 & 0.55 & 2.4 & 34 & 18 & 46 \\
\hline Amargosa River East of Death Valley Junction & JY05-37b & 860 & 14 & 3,100 & 0.97 & 4.0 & 78 & 21 & 89 \\
\hline Amargosa River East of Death Valley Junction & JY05-37c & 670 & 15 & 3,200 & 0.81 & 2.9 & 61 & 25 & 78 \\
\hline Amargosa River East of Death Valley Junction & JY05-38a & 460 & 13 & 2,000 & 0.67 & 2.9 & 32 & 22 & 48 \\
\hline Ash Meadows & JY03-49a/49b & 1,300 & 7.1 & 1,400 & 0.63 & 1.9 & 29 & 14 & 39 \\
\hline Ash Meadows & $J Y 03-50 a / 50 b$ & 1,300 & 5.3 & 1,400 & 0.59 & 1.7 & 23 & 15 & 30 \\
\hline Ash Meadows & JY05-29 & 410 & 2.6 & 500 & 0.30 & 53 & 24 & 4.5 & 12 \\
\hline Clay Dune & JY05-25 & 810 & 9.1 & 2,400 & 0.67 & 1.6 & 46 & 16 & 71 \\
\hline Coppice & JY05-17 & 590 & 11 & 2,500 & 0.65 & 3.5 & 46 & 21 & 61 \\
\hline Discovery & JY05-15 & 980 & 12 & 2,500 & 0.67 & 2.7 & 46 & 22 & 60 \\
\hline Ojo del Caballo & JY05-30 & 520 & 5.0 & 1,100 & 0.32 & 8.8 & 32 & 11 & 16 \\
\hline Salt Pan & JY03-43a & 800 & 8.4 & 2,100 & 0.59 & 1.4 & 44 & 17 & 67 \\
\hline Salt Pan & JY03-43b & 640 & 7.2 & 2,600 & 0.56 & 1.5 & 45 & 15 & 65 \\
\hline Salt Pan & JY03-43c & 660 & 8.4 & 2,600 & 0.59 & 1.5 & 45 & 16 & 65 \\
\hline Salt Pan & JY05-23a & 200 & 4.1 & 660 & 0.17 & 0.85 & 9.9 & 5.8 & 9 \\
\hline Salt Pan & JY05-59a & 60 & 0.69 & 200 & $<0.08$ & 0.27 & 3.1 & 1.7 & 4 \\
\hline Salt Pan & JY05-59b & 270 & 3.1 & 890 & 0.16 & 0.61 & 14 & 7.3 & 17 \\
\hline West Transect & JY05-19 & 810 & 14 & 2,300 & 0.82 & 2.8 & 44 & 22 & 67 \\
\hline
\end{tabular}


Table 11C. Geochemical data determined on the fine fraction of selected grab samples. Method of determination (ICP-MS, inductively coupled plasma-mass spectrometry; ICP-OES, inductively coupled plasma-optical emission spectrometry) is listed above each element.

[Sample site, name of sampling site; Sample ID, unique sample identifier; ppm, parts per million; wt.\%, weight percent]

\begin{tabular}{|c|c|c|c|c|c|c|c|c|c|c|c|}
\hline Sample site & Sample ID & $\begin{array}{l}\text { ICP-MS } \\
\text { Ag ppm }\end{array}$ & $\begin{array}{l}\text { ICP-OES } \\
\text { Al wt. } \%\end{array}$ & $\begin{array}{l}\text { ICP-MS } \\
\text { As ppm }\end{array}$ & $\begin{array}{l}\text { ICP-MS } \\
\text { Ba ppm }\end{array}$ & $\begin{array}{l}\text { ICP-MS } \\
\text { Be ppm }\end{array}$ & $\begin{array}{c}\text { ICP-MS } \\
\text { Bi ppm }\end{array}$ & $\begin{array}{l}\text { ICP-OES } \\
\text { Ca wt. } \%\end{array}$ & $\begin{array}{l}\text { ICP-MS } \\
\text { Cd ppm }\end{array}$ & $\begin{array}{l}\text { ICP-MS } \\
\text { Ce ppm }\end{array}$ & $\begin{array}{l}\text { ICP-MS } \\
\text { Co ppm }\end{array}$ \\
\hline Ash Meadows & JY03-49a & $<2$ & 5.1 & 15 & 570 & 1.6 & 0.12 & 17 & 0.13 & 61 & 5.3 \\
\hline Ash Meadows & JY03-50a & $<2$ & 4.2 & 11 & 590 & 1.4 & 0.09 & 17 & 0.12 & 48 & 3.8 \\
\hline Coppice & JY04-15a & 2.2 & 5.7 & 23 & 540 & 2.3 & 0.20 & 5.6 & 0.27 & 75 & 9.4 \\
\hline Coppice & JY04-16a & 2.2 & 6.5 & 14 & 610 & 2.8 & 0.21 & 6.4 & 0.32 & 82 & 9.6 \\
\hline Discovery & JY03-01 & $<3$ & 5.4 & 22 & 650 & 1.9 & 0.14 & 8.5 & 0.23 & 72 & 7.0 \\
\hline Discovery & JY03-01a & $<3$ & 5.9 & 11 & 830 & 2.1 & 0.16 & 9.9 & 0.25 & 86 & 7.5 \\
\hline Discovery & JY03-01b & $<3$ & 5.0 & 34 & 540 & 1.7 & 0.16 & 5.1 & 0.21 & 59 & 7.8 \\
\hline Franklin Lake Playa/Carson Slough & JY04-01 & $<2$ & 0.087 & 36 & 15 & 0.03 & $<0.06$ & 0.33 & 0.02 & 3.3 & 0.84 \\
\hline Salt Pan & JY03-25a & $<2$ & 5.0 & 13 & 520 & 1.6 & 0.15 & 7.3 & 0.17 & 71 & 6.9 \\
\hline Salt Pan & JY03-25b & $<2$ & 6.4 & 16 & 450 & 2.2 & 0.20 & 7.0 & 0.18 & 56 & 10 \\
\hline Salt Pan & JY03-25c & $<2$ & 5.9 & 18 & 390 & 2.1 & 0.18 & 5.4 & 0.14 & 47 & 9.9 \\
\hline Salt Pan & JY03-43a & $<2$ & 6.0 & 12 & 590 & 1.9 & 0.16 & 10 & 0.21 & 70 & 7.7 \\
\hline Salt Pan & JY03-43b & $<2$ & 5.8 & 37 & 380 & 2.0 & 0.19 & 5.7 & 0.16 & 47 & 9.8 \\
\hline Salt Pan & JY03-43c & $<2$ & 6.5 & 36 & 440 & 2.2 & 0.20 & 6.4 & 0.17 & 55 & 11 \\
\hline Sample site & Sample ID & $\begin{array}{l}\text { ICP-MS } \\
\text { Cr ppm }\end{array}$ & $\begin{array}{l}\text { ICP-MS } \\
\text { Cs ppm }\end{array}$ & $\begin{array}{l}\text { ICP-MS } \\
\text { Cu ppm }\end{array}$ & $\begin{array}{l}\text { ICP-OES } \\
\text { Fe wt. } \%\end{array}$ & $\begin{array}{l}\text { ICP-MS } \\
\text { Ga ppm }\end{array}$ & $\begin{array}{c}\text { ICP-OES } \\
\text { K wt. } \%\end{array}$ & $\begin{array}{l}\text { ICP-MS } \\
\text { La ppm }\end{array}$ & $\begin{array}{c}\text { ICP-MS } \\
\text { Li ppm }\end{array}$ & $\begin{array}{l}\text { ICP-OES } \\
\text { Mg wt. } \%\end{array}$ & $\begin{array}{l}\text { ICP-MS } \\
\text { Mn ppm }\end{array}$ \\
\hline Ash Meadows & JY03-49a & 28 & 7.4 & 12 & 1.6 & 9.8 & 2.7 & 35 & 130 & 4.2 & 420 \\
\hline Ash Meadows & JY03-50a & 22 & 4.7 & 9.6 & 1.2 & 8.0 & 2.0 & 26 & 73 & 2.7 & 340 \\
\hline Coppice & JY04-15a & 47 & 8.8 & 27 & 2.5 & 13 & 2.6 & 42 & 100 & 2.1 & 660 \\
\hline Coppice & JY04-16a & 49 & 9.3 & 26 & 2.8 & 16 & 2.9 & 49 & 110 & 2.4 & 790 \\
\hline Discovery & JY03-01 & 36 & 6.4 & 19 & 1.8 & 13 & 2.6 & 38 & 86 & 2.0 & 500 \\
\hline Discovery & JY03-01a & 53 & 6.4 & 19 & 2.0 & 14 & 2.7 & 46 & 84 & 2.0 & 530 \\
\hline Discovery & JY03-01b & 41 & 6.7 & 19 & 1.9 & 12 & 2.4 & 35 & 87 & 1.8 & 570 \\
\hline Franklin Lake Playa/Carson Slough & JY04-01 & 6.9 & 0.11 & 11 & 0.046 & 0.23 & 0.59 & 1.4 & 5.5 & 0.030 & 10 \\
\hline Salt Pan & JY03-25a & 32 & 5.6 & 16 & 2.1 & 11 & 2.4 & 42 & 140 & 2.3 & 470 \\
\hline Salt Pan & JY03-25b & 35 & 10 & 24 & 2.7 & 14 & 3.4 & 34 & 330 & 4.6 & 700 \\
\hline Salt Pan & JY03-25c & 31 & 10 & 24 & 2.6 & 13 & 3.2 & 29 & 360 & 4.3 & 670 \\
\hline Salt Pan & JY03-43a & 29 & 6.7 & 17 & 2.2 & 12 & 2.9 & 42 & 190 & 2.9 & 530 \\
\hline Salt Pan & JY03-43b & 32 & 10 & 24 & 2.5 & 13 & 3.3 & 30 & 580 & 4.4 & 670 \\
\hline Salt Pan & JY03-43c & 36 & 11 & 25 & 2.8 & 14 & 3.6 & 33 & 600 & 4.6 & 730 \\
\hline Sample site & Sample ID & $\begin{array}{l}\text { ICP-MS } \\
\text { Mo ppm }\end{array}$ & $\begin{array}{l}\text { ICP-OES } \\
\text { Na wt. } \%\end{array}$ & $\begin{array}{l}\text { ICP-MS } \\
\text { Nb ppm }\end{array}$ & $\begin{array}{l}\text { ICP-O } \\
\text { Nd pI }\end{array}$ & $\begin{array}{l}\text { ICP } \\
\mathrm{Ni}\end{array}$ & $\begin{array}{l}\text {-MS } \\
\text { opm }\end{array}$ & $\begin{array}{l}\text { CP-MS } \\
\text { P ppm }\end{array}$ & $\begin{array}{l}\text { ICP-MS } \\
\text { Pb ppm }\end{array}$ & $\begin{array}{l}\text { ICP-MS } \\
\text { Rb ppm }\end{array}$ & $\begin{array}{l}\text { ICP-MS } \\
\text { Sb ppm }\end{array}$ \\
\hline Ash Meadows & JY03-49a & 0.55 & 2.7 & 9.2 & 25 & & 9.6 & 550 & 12 & 91 & 0.73 \\
\hline Ash Meadows & JY03-50a & 0.72 & 5.8 & 6.4 & 23 & & 5.0 & 510 & 11 & 65 & 0.58 \\
\hline Coppice & JY04-15a & 6.7 & 5.8 & 27 & 26 & 2 & & 810 & 22 & 110 & 1.4 \\
\hline Coppice & JY04-16a & 1.2 & 3.0 & 25 & 30 & 2 & & 680 & 23 & 130 & 1.6 \\
\hline Discovery & JY03-01 & 5.8 & 5.8 & 21 & 26 & 1 & & 690 & 18 & 110 & 0.98 \\
\hline Discovery & JY03-01a & 1.2 & 3.0 & 24 & 31 & 1 & & 680 & 21 & 110 & 1.1 \\
\hline Discovery & JY03-01b & 7.1 & 9.4 & 23 & 23 & 1 & & 960 & 17 & 97 & 1.0 \\
\hline Franklin Lake Playa/Carson Slough & JY04-01 & 16 & 30 & 0.64 & $<4$ & & 1.6 & 130 & 0.88 & 3.5 & 0.22 \\
\hline Salt Pan & JY03-25a & 0.87 & 9.5 & 11 & 32 & 1 & & 520 & 15 & 84 & 0.91 \\
\hline Salt Pan & JY03-25b & 0.78 & 4.5 & 13 & 27 & 1 & & 460 & 17 & 130 & 1.3 \\
\hline Salt Pan & JY03-25c & 0.97 & 7.1 & 12 & 24 & 1 & & 390 & 15 & 120 & 1.2 \\
\hline Salt Pan & JY03-43a & 0.69 & 5.4 & 12 & 37 & 1 & & 490 & 17 & 98 & 1.0 \\
\hline Salt Pan & JY03-43b & 5.1 & 6.3 & 12 & 23 & 1 & & 420 & 15 & 120 & 1.2 \\
\hline Salt Pan & JY03-43c & 3.3 & 5.1 & 14 & 26 & 1 & & 480 & 16 & 130 & 1.2 \\
\hline Sample site & Sample ID & $\begin{array}{l}\text { ICP-MS } \\
\text { Sc ppm }\end{array}$ & $\begin{array}{l}\text { ICP-MS } \\
\text { Sr ppm }\end{array}$ & $\begin{array}{c}\text { ICP-MS } \\
\text { Th ppm }\end{array}$ & $\begin{array}{l}\text { ICP- } \\
\text { Ti p }\end{array}$ & & $\begin{array}{l}\text {-Mpm } \\
\text { ppm }\end{array}$ & $\begin{array}{l}\text { CP-MS } \\
\text { U ppm }\end{array}$ & $\begin{array}{c}\text { ICP-MS } \\
\text { V ppm }\end{array}$ & $\begin{array}{c}\text { ICP-MS } \\
\text { Y ppm }\end{array}$ & $\begin{array}{l}\text { ICP-MS } \\
\text { Zn ppm }\end{array}$ \\
\hline Ash Meadows & JY03-49a & 6.8 & 1,800 & 9.8 & 2,4 & & .74 & 1.8 & 40 & 19 & 43 \\
\hline Ash Meadows & JY03-50a & 5.6 & 1,700 & 7.8 & 2,0 & & .63 & 1.6 & 30 & 17 & 31 \\
\hline Coppice & JY04-15a & 8.2 & 690 & 12 & 3,2 & & .68 & 2.7 & 57 & 22 & 77 \\
\hline Coppice & JY04-16a & 9.2 & 810 & 14 & 4,1 & & .78 & 2.4 & 59 & 26 & 88 \\
\hline Discovery & JY03-01 & 7.3 & 1,000 & 13 & 3,0 & & .65 & 3.2 & 45 & 21 & 58 \\
\hline Discovery & JY03-01a & 7.7 & 1,200 & 14 & 3,4 & & .70 & 2.5 & 48 & 25 & 77 \\
\hline Discovery & JY03-01b & 7.6 & 670 & 11 & 3,1 & & .67 & 6.7 & 49 & 19 & 63 \\
\hline Franklin Lake Playa/Carson Slough & JY04-01 & 0.2 & 47 & 0.55 & & $<0$ & .08 & 17 & 8.5 & 0.57 & 4.9 \\
\hline Salt Pan & JY03-25a & 7.4 & 980 & 12 & 3,0 & & .52 & 1.8 & 50 & 24 & 51 \\
\hline Salt Pan & JY03-25b & 8.9 & 940 & 11 & 3,0 & & .69 & 1.9 & 55 & 21 & 79 \\
\hline Salt Pan & JY03-25c & 8.6 & 790 & 9.1 & 2,8 & & .63 & 1.6 & 53 & 20 & 75 \\
\hline Salt Pan & JY03-43a & 8.1 & 1,300 & 13 & 3,0 & & .55 & 1.7 & 48 & 27 & 56 \\
\hline Salt Pan & JY03-43b & 8.8 & 840 & 10 & 2,8 & & .64 & 1.8 & 51 & 19 & 74 \\
\hline Salt Pan & JY03-43c & 9.5 & 890 & 11 & 3,0 & & .72 & 1.7 & 55 & 21 & 79 \\
\hline
\end{tabular}


Table 12A. Composition of the DI-leach bulk East transect auger hole samples. Method of determination (ICP-MS, inductively coupled plasma-mass spectrometry; ICP-OES, inductively coupled plasma-optical emission spectrometry; IC, ion chromatograph) is listed above each element.

[Sample site, name of sampling site; Sample ID, unique sample identifier; Treatment, type of sample preparation; d, duplicate; $\mu \mathrm{g} / \mathrm{L}, \mathrm{micrograms}$ per liter; $\mathrm{mg} / \mathrm{L}$, milligrams per liter; $\mathrm{mg} / \mathrm{L} \mathrm{CaCO}_{3}$, milligrams per liter calcium carbonate]

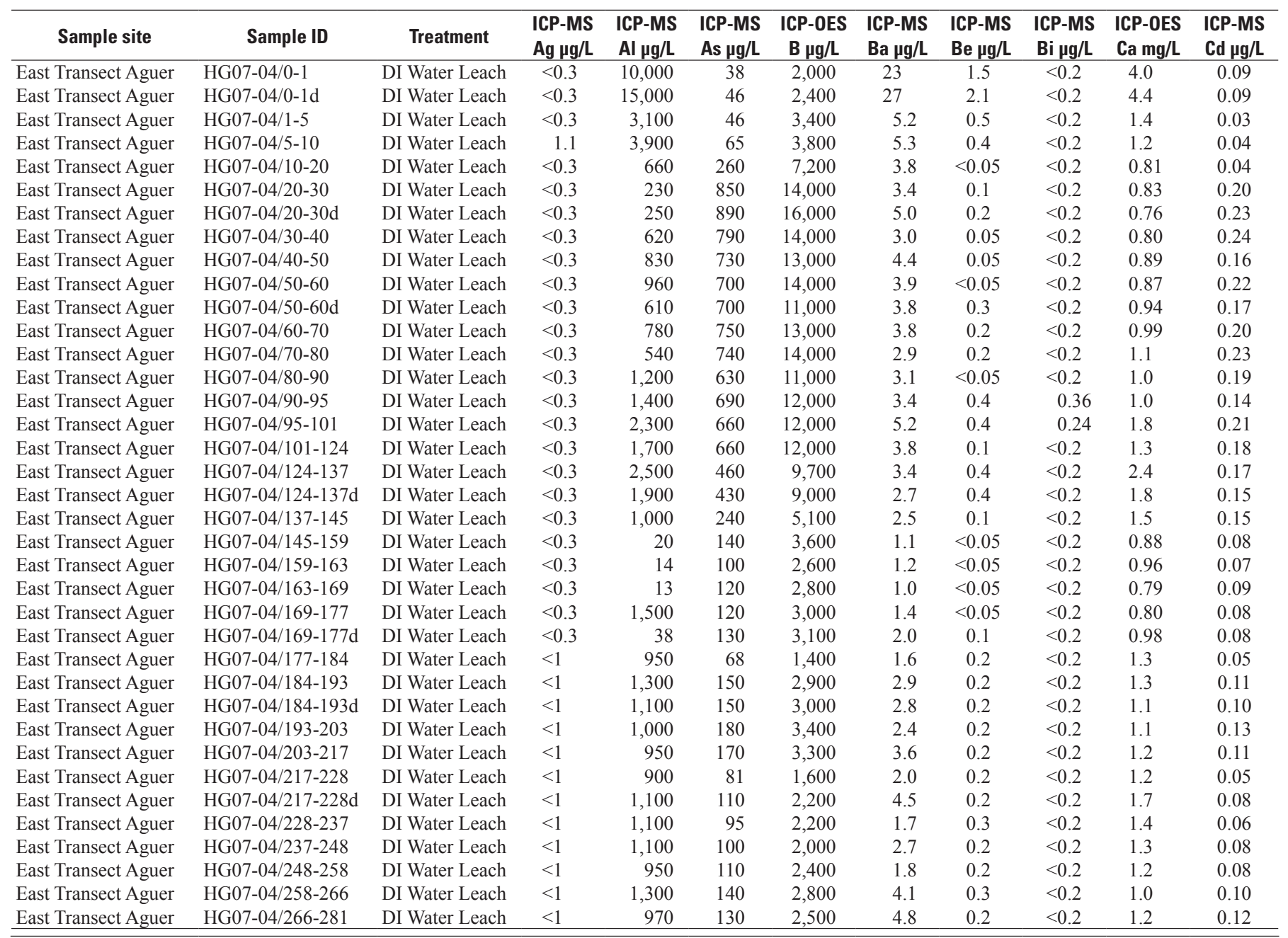


Table 12A. Composition of the DI-leach bulk East transect auger hole samples. Method of determination (ICP-MS, inductively coupled plasma-mass spectrometry; ICP-OES, inductively coupled plasma-optical emission spectrometry; IC, ion chromatograph) is listed above each element.-Continued

[Sample site, name of sampling site; Sample ID, unique sample identifier; Treatment, type of sample preparation; d, duplicate; $\mu \mathrm{g} / \mathrm{L}, \mathrm{micrograms}$ per liter; $\mathrm{mg} / \mathrm{L}$, milligrams per liter; $\mathrm{mg} / \mathrm{L} \mathrm{CaCO}_{3}$, milligrams per liter calcium carbonate]

\begin{tabular}{|c|c|c|c|c|c|c|c|c|c|c|c|}
\hline Sample site & Sample ID & $\begin{array}{l}\text { ICP-MS } \\
\text { Ce } \mu g / L\end{array}$ & $\begin{array}{l}\text { ICP-MS } \\
\text { Co } \mu g / L\end{array}$ & $\begin{array}{l}\text { ICP-MS } \\
\text { Cr } \mu g / L\end{array}$ & $\begin{array}{l}\text { ICP-MS } \\
\text { Cs } \mu g / L\end{array}$ & $\begin{array}{l}\text { ICP-MS } \\
\text { Cu } \mu g / L\end{array}$ & $\begin{array}{l}\text { ICP-MS } \\
\text { Dy } \mu \mathrm{g} / \mathrm{L}\end{array}$ & $\begin{array}{c}\text { ICP-MS } \\
\text { Er } \mu g / L\end{array}$ & $\begin{array}{l}\text { ICP-MS } \\
\text { Eu } \mu g / L\end{array}$ & $\begin{array}{c}\text { ICP-0ES } \\
\mathrm{Fe} \mu \mathrm{g} / \mathrm{L}\end{array}$ & $\begin{array}{l}\text { ICP-MS } \\
\text { Ga } \mu g / L\end{array}$ \\
\hline East Transect Aguer & HG07-04/0-1d & 2.7 & 12 & 13 & 3.7 & 39 & 0.097 & 0.040 & 0.009 & 12,000 & 8.5 \\
\hline East Transect Aguer & HG07-04/1-5 & 1.1 & 2.7 & 3.8 & 0.82 & 8.000 & 0.005 & 0.010 & $<0.005$ & 3,200 & 1.6 \\
\hline East Transect Aguer & HG07-04/10-20 & 0.23 & 0.42 & 7.5 & 0.28 & 4.400 & 0.020 & 0.005 & 0.008 & 560 & 0.37 \\
\hline East Transect Aguer & HG07-04/20-30 & 0.24 & 0.48 & 16 & 0.14 & 7.700 & 0.009 & 0.020 & 0.008 & 180 & 0.10 \\
\hline East Transect Aguer & HG07-04/20-30d & 0.29 & 0.53 & 17 & 0.17 & 6.500 & 0.010 & 0.020 & $<0.005$ & 180 & 0.10 \\
\hline East Transect Aguer & HG07-04/50-60 & 0.19 & 0.51 & 10 & 0.38 & 3.900 & 0.006 & 0.005 & 0.008 & 700 & 0.41 \\
\hline East Transect Aguer & HG07-04/50-60d & 0.15 & 0.42 & 9.4 & 0.26 & 2.800 & $<0.005$ & 0.005 & 0.010 & 320 & 0.22 \\
\hline East Transect Aguer & HG07-04/60-70 & 0.21 & 0.50 & 11 & 0.31 & 3.800 & $<0.005$ & 0.008 & $<0.005$ & 540 & 0.28 \\
\hline East Transect Aguer & HG07-04/70-80 & 0.18 & 0.43 & 9.7 & 0.23 & 2.800 & 0.006 & $<0.005$ & $<0.005$ & 410 & 0.20 \\
\hline East Transect Aguer & HG07-04/80-90 & 0.29 & 0.65 & 8.3 & 0.46 & 4.700 & 0.009 & 0.008 & 0.010 & 690 & 0.44 \\
\hline East Transect Aguer & HG07-04/90-95 & 0.38 & 0.64 & 9.1 & 0.63 & 3.300 & 0.006 & $<0.005$ & $<0.005$ & 930 & 0.55 \\
\hline East Transect Aguer & HG07-04/145-159 & 0.02 & 0.03 & 1.3 & 0.06 & 4.200 & $<0.005$ & $<0.005$ & $<0.005$ & 22 & $<0.05$ \\
\hline East Transect Aguer & HG07-04/159-163 & 0.02 & 0.03 & $<1$ & 0.07 & 3.300 & $<0.005$ & $<0.005$ & 0.005 & $<20$ & $<0.05$ \\
\hline East Transect Aguer & HG07-04/163-169 & 0.02 & $<0.02$ & $<1$ & 0.06 & 3.100 & 0.006 & $<0.005$ & $<0.005$ & 25 & $<0.05$ \\
\hline East Transect Aguer & HG07-04/169-177 & 0.02 & 0.03 & $<1$ & 0.09 & 3.500 & 0.005 & $<0.005$ & $<0.005$ & $<20$ & $<0.05$ \\
\hline East Transect Aguer & HG07-04/169-177d & 0.03 & 0.03 & $<1$ & 0.10 & 5.100 & $<0.005$ & $<0.005$ & $<0.005$ & 21 & $<0.05$ \\
\hline East Transect Aguer & HG07-04/177-184 & 0.92 & 0.54 & 1.7 & 1.1 & 1.900 & 0.040 & 0.020 & $<0.005$ & 1,600 & 0.43 \\
\hline East Transect Aguer & HG07-04/184-193 & 0.60 & 0.63 & 3.2 & 1.7 & 2.900 & 0.020 & 0.010 & $<0.005$ & 2,100 & 0.54 \\
\hline East Transect Aguer & HG07-04/184-193d & 0.51 & 0.55 & 2.4 & 1.6 & 2.600 & 0.010 & 0.010 & $<0.005$ & 2,000 & 0.48 \\
\hline East Transect Aguer & HG07-04/193-203 & 0.49 & 0.50 & 3.3 & 1.6 & 2.800 & 0.010 & 0.010 & $<0.005$ & 1,900 & 0.46 \\
\hline East Transect Aguer & HG07-04/203-217 & 0.64 & 0.56 & 3.1 & 1.7 & 3.600 & 0.020 & 0.010 & $<0.005$ & 1,900 & 0.42 \\
\hline East Transect Aguer & HG07-04/217-228 & 1.1 & 0.61 & 2.1 & 1.2 & 3.600 & 0.040 & 0.020 & 0.005 & 2,300 & 0.43 \\
\hline East Transect Aguer & HG07-04/217-228d & 0.79 & 0.63 & 2.4 & 1.3 & 3.200 & 0.030 & 0.020 & $<0.005$ & 1,700 & 0.45 \\
\hline East Transect Aguer & HG07-04/228-237 & 1.2 & 0.76 & 2.4 & 1.2 & 2.900 & 0.040 & 0.030 & $<0.005$ & 3,000 & 0.46 \\
\hline
\end{tabular}


Table 12A. Composition of the DI-leach bulk East transect auger hole samples. Method of determination (ICP-MS, inductively coupled plasma-mass spectrometry; ICP-OES, inductively coupled plasma-optical emission spectrometry; IC, ion chromatograph) is listed above each element.-Continued

[Sample site, name of sampling site; Sample ID, unique sample identifier; Treatment, type of sample preparation; d, duplicate; $\mu \mathrm{g} / \mathrm{L}, \mathrm{micrograms}$ per liter; $\mathrm{mg} / \mathrm{L}$, milligrams per liter; $\mathrm{mg} / \mathrm{L} \mathrm{CaCO}_{3}$, milligrams per liter calcium carbonate]

\begin{tabular}{|c|c|c|c|c|c|c|c|c|c|c|c|c|}
\hline Sample site & Sample ID & $\begin{array}{l}\text { ICP-MS } \\
\text { Gd } \mu g / L\end{array}$ & $\begin{array}{l}\text { ICP-MS } \\
\text { Ge } \mu \mathrm{g} / \mathrm{L}\end{array}$ & $\begin{array}{l}\text { ICP-MS } \\
\text { Ho } \mu \mathrm{g} / \mathrm{L}\end{array}$ & $\begin{array}{c}\text { ICP-OES } \\
\mathrm{K} \mathrm{mg} / \mathrm{L}\end{array}$ & $\begin{array}{l}\text { ICP-MS } \\
\text { La } \mu \mathrm{g} / \mathrm{L}\end{array}$ & $\begin{array}{c}\text { ICP-OES } \\
\mathrm{Li} \mu \mathrm{g} / \mathrm{L}\end{array}$ & $\begin{array}{l}\text { ICP-MS } \\
\text { Lu } \mu g / L\end{array}$ & $\begin{array}{l}\text { ICP-MS } \\
\text { Mg mg/L }\end{array}$ & $\begin{array}{l}\text { ICP-MS } \\
\text { Mn } \mu \mathrm{g} / \mathrm{L}\end{array}$ & $\begin{array}{l}\text { ICP-MS } \\
\text { Mo } \mu \mathrm{g} / \mathrm{L} \\
\end{array}$ & $\begin{array}{l}\text { ICP-OES } \\
\text { Na mg/L }\end{array}$ \\
\hline East Transect Aguer & HG07-04/0-1d & 0.091 & 2.5 & 0.020 & 16 & 0.37 & 520 & $<0.1$ & 110 & 800 & $<2$ & 240 \\
\hline East Transect Aguer & HG07-04/1-5 & $<0.005$ & 0.49 & $<0.005$ & 13 & 0.12 & 110 & $<0.1$ & 21 & 210 & $<2$ & 510 \\
\hline East Transect Aguer & HG07-04/10-20 & $<0.005$ & 0.1 & $<0.005$ & 19 & 0.02 & 19 & $<0.1$ & 2.6 & 30 & 30 & 880 \\
\hline East Transect Aguer & HG07-04/20-30 & 0.030 & $<0.05$ & $<0.005$ & 25 & 0.04 & 9.6 & $<0.1$ & 0.84 & 11 & 140 & 1,100 \\
\hline East Transect Aguer & HG07-04/20-30d & 0.010 & $<0.05$ & $<0.005$ & 25 & 0.05 & 8.9 & $<0.1$ & 0.85 & 11 & 140 & 1,000 \\
\hline East Transect Aguer & HG07-04/50-60 & $<0.005$ & $<0.05$ & $<0.005$ & 19 & 0.02 & 21 & $<0.1$ & 2.8 & 37 & 130 & 670 \\
\hline East Transect Aguer & HG07-04/50-60d & $<0.005$ & $<0.05$ & $<0.005$ & 19 & 0.06 & 17 & $<0.1$ & 1.9 & 25 & 130 & 670 \\
\hline East Transect Aguer & HG07-04/60-70 & $<0.005$ & 0.06 & $<0.005$ & 19 & 0.06 & 16 & $<0.1$ & 2.2 & 30 & 140 & 610 \\
\hline East Transect Aguer & HG07-04/70-80 & 0.007 & $<0.05$ & $<0.005$ & 19 & 0.03 & 12 & $<0.1$ & 1.9 & 24 & 140 & 610 \\
\hline East Transect Aguer & HG07-04/80-90 & 0.020 & 0.08 & $<0.005$ & 16 & 0.03 & 25 & $<0.1$ & 4.0 & 50 & 120 & 500 \\
\hline East Transect Aguer & HG07-04/90-95 & 0.020 & 0.20 & $<0.005$ & 16 & 0.08 & 25 & $<0.1$ & 4.2 & 53 & 130 & 530 \\
\hline East Transect Aguer & HG07-04/145-159 & $<0.005$ & 0.06 & $<0.005$ & 6.4 & $<0.01$ & 3.2 & $<0.1$ & 0.05 & 0.3 & 46 & 210 \\
\hline East Transect Aguer & HG07-04/159-163 & 0.010 & $<0.05$ & $<0.005$ & 5.4 & $<0.01$ & 4.9 & $<0.1$ & 0.12 & 1.2 & 36 & 170 \\
\hline East Transect Aguer & HG07-04/163-169 & 0.010 & $<0.05$ & $<0.005$ & 5.5 & $<0.01$ & $<0.9$ & $<0.1$ & 0.08 & 0.8 & 41 & 170 \\
\hline East Transect Aguer & HG07-04/169-177 & $<0.005$ & 0.08 & $<0.005$ & 6.5 & $<0.01$ & 4.7 & $<0.1$ & 0.04 & $<0.2$ & 49 & 220 \\
\hline East Transect Aguer & HG07-04/169-177d & 0.030 & 0.10 & $<0.005$ & 6.8 & $<0.01$ & 5.3 & $<0.1$ & 0.15 & 1.8 & 50 & 220 \\
\hline East Transect Aguer & HG07-04/177-184 & 0.030 & 0.44 & 0.006 & 4.4 & 0.23 & 65 & $<0.1$ & 6.3 & 41 & 29 & 130 \\
\hline East Transect Aguer & HG07-04/184-193 & 0.020 & 0.52 & $<0.005$ & 6.6 & 0.09 & 83 & $<0.1$ & 11 & 110 & 66 & 240 \\
\hline East Transect Aguer & HG07-04/184-193d & 0.010 & 0.46 & $<0.005$ & 6.2 & 0.06 & 72 & $<0.1$ & 9.5 & 92 & 65 & 240 \\
\hline East Transect Aguer & HG07-04/193-203 & 0.010 & 0.42 & $<0.005$ & 7.9 & 0.05 & 86 & $<0.1$ & 9.6 & 91 & 79 & 290 \\
\hline East Transect Aguer & HG07-04/203-217 & 0.020 & 0.45 & $<0.005$ & 7.7 & 0.07 & 80 & $<0.1$ & 9.0 & 63 & 80 & 270 \\
\hline East Transect Aguer & HG07-04/217-228 & 0.040 & 0.48 & 0.007 & 3.8 & 0.3 & 64 & $<0.1$ & 6.7 & 47 & 33 & 140 \\
\hline East Transect Aguer & HG07-04/217-228d & 0.030 & 0.42 & 0.005 & 4.9 & 0.18 & 88 & $<0.1$ & 10 & 99 & 43 & 180 \\
\hline East Transect Aguer & HG07-04/228-237 & 0.040 & 0.52 & 0.007 & 6.5 & 0.24 & 110 & $<0.1$ & 9.1 & 59 & 36 & 190 \\
\hline
\end{tabular}


Table 12A. Composition of the DI-leach bulk East transect auger hole samples. Method of determination (ICP-MS, inductively coupled plasma-mass spectrometry; ICP-OES, inductively coupled plasma-optical emission spectrometry; IC, ion chromatograph) is listed above each element.-Continued

[Sample site, name of sampling site; Sample ID, unique sample identifier; Treatment, type of sample preparation; d, duplicate; $\mu \mathrm{g} / \mathrm{L}, \mathrm{micrograms} \mathrm{per} \mathrm{liter;} \mathrm{mg} / \mathrm{L}$, milligrams per liter; $\mathrm{mg} / \mathrm{L} \mathrm{CaCO}_{3}$, milligrams per liter calcium carbonate]

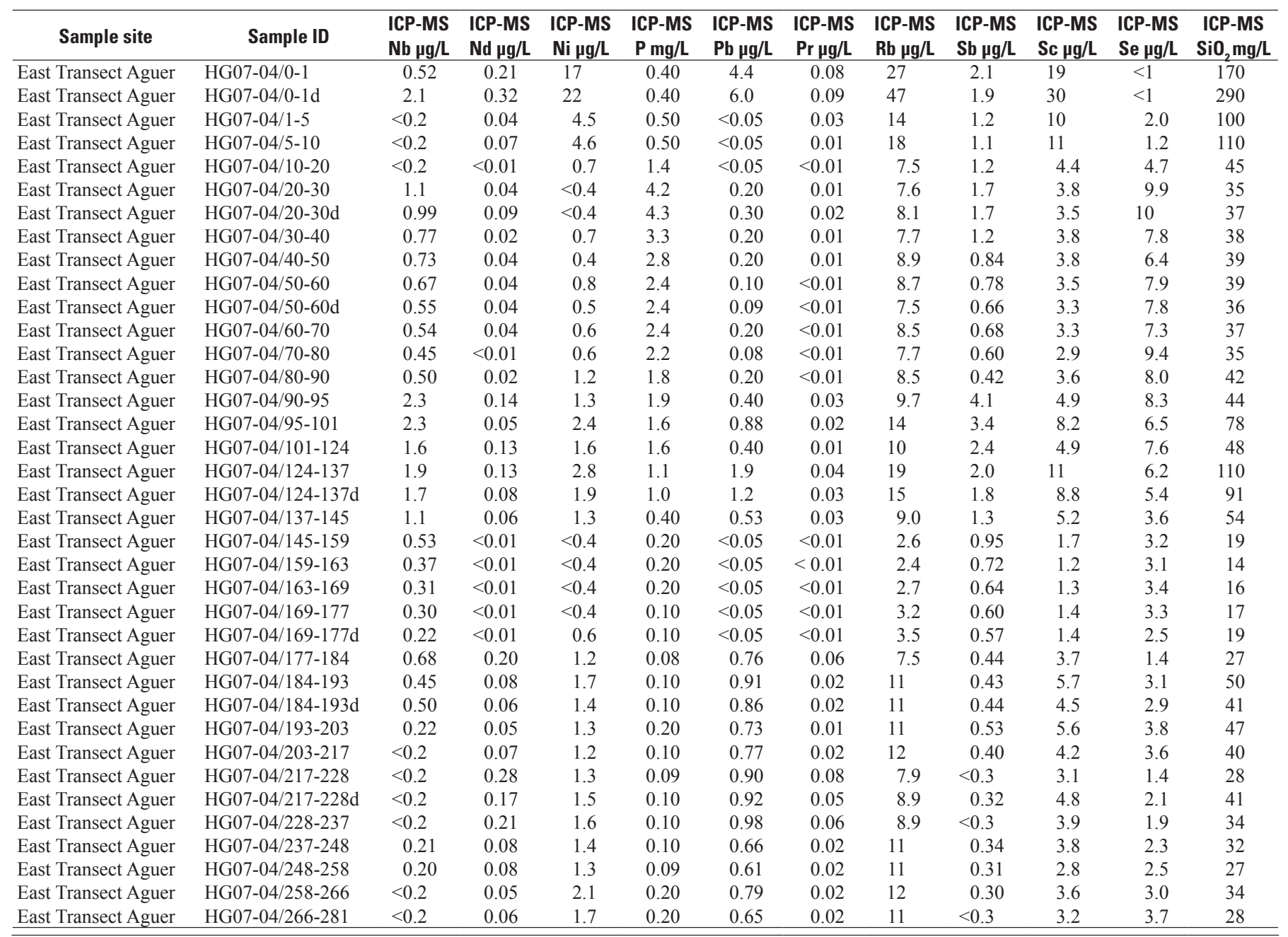


Table 12A. Composition of the DI-leach bulk East transect auger hole samples. Method of determination (ICP-MS, inductively coupled plasma-mass spectrometry; ICP-OES, inductively coupled plasma-optical emission spectrometry; IC, ion chromatograph) is listed above each element.-Continued

[Sample site, name of sampling site; Sample ID, unique sample identifier; Treatment, type of sample preparation; d, duplicate; $\mu \mathrm{g} / \mathrm{L}, \mathrm{micrograms}$ per liter; $\mathrm{mg} / \mathrm{L}$, milligrams per liter; $\mathrm{mg} / \mathrm{L} \mathrm{CaCO}_{3}$, milligrams per liter calcium carbonate]

\begin{tabular}{|c|c|c|c|c|c|c|c|c|c|c|c|c|}
\hline Sample site & Sample ID & $\begin{array}{l}\text { ICP-MS } \\
\text { Sm } \mu g / L\end{array}$ & $\begin{array}{c}\text { ICP-0ES } \\
\text { Sr } \mu \mathrm{g} / \mathrm{L}\end{array}$ & $\begin{array}{l}\text { ICP-MS } \\
\text { Ta } \mu g / L\end{array}$ & $\begin{array}{l}\text { ICP-MS } \\
\text { Tb } \mu \mathrm{g} / \mathrm{L}\end{array}$ & $\begin{array}{l}\text { ICP-MS } \\
\text { Th } \mu \mathrm{g} / \mathrm{L}\end{array}$ & $\begin{array}{c}\text { ICP-MS } \\
\text { Ti } \mu \mathrm{g} / \mathrm{L}\end{array}$ & $\begin{array}{l}\text { ICP-MS } \\
\text { TI } \mu \mathrm{g} / \mathrm{L}\end{array}$ & $\begin{array}{l}\text { ICP-MS } \\
\mathrm{Tm} \mu \mathrm{g} / \mathrm{L}\end{array}$ & $\begin{array}{c}\text { ICP-MS } \\
U \mu \mathrm{g} / \mathrm{L}\end{array}$ & $\begin{array}{c}\text { ICP-MS } \\
V \mu \mathrm{g} / \mathrm{L}\end{array}$ & $\begin{array}{l}\text { ICP-MS } \\
\text { W } \mu g / L\end{array}$ \\
\hline East Transect Aguer & HG07-04/0-1d & 0.08 & 120 & 0.90 & 0.010 & $<0.2$ & 390 & 0.2 & 0.020 & $<0.1$ & 130 & 1.7 \\
\hline East Transect Aguer & HG07-04/1-5 & $<0.01$ & 72 & 0.66 & 0.005 & $<0.2$ & 65 & $<0.1$ & $<0.005$ & 0.28 & 190 & 1.1 \\
\hline East Transect Aguer & HG07-04/10-20 & 0.01 & 36 & 0.50 & $<0.005$ & $<0.2$ & 26 & $<0.1$ & $<0.005$ & 9.8 & 240 & 8.4 \\
\hline East Transect Aguer & HG07-04/20-30 & 0.01 & 29 & 0.34 & $<0.005$ & $<0.2$ & 15 & $<0.1$ & $<0.005$ & 100 & 310 & 14 \\
\hline East Transect Aguer & HG07-04/20-30d & 0.02 & 30 & 0.29 & $<0.005$ & $<0.2$ & 15 & $<0.1$ & $<0.005$ & 100 & 320 & 15 \\
\hline East Transect Aguer & HG07-04/50-60 & 0.01 & 59 & 0.20 & $<0.005$ & $<0.2$ & 32 & $<0.1$ & $<0.005$ & 28 & 300 & 9.4 \\
\hline East Transect Aguer & HG07-04/50-60d & 0.01 & 64 & 0.20 & $<0.005$ & $<0.2$ & 22 & $<0.1$ & $<0.005$ & 28 & 290 & 9.4 \\
\hline East Transect Aguer & HG07-04/60-70 & 0.02 & 63 & 0.20 & $<0.005$ & $<0.2$ & 26 & $<0.1$ & $<0.005$ & 26 & 290 & 10 \\
\hline East Transect Aguer & HG07-04/70-80 & 0.01 & 67 & 0.08 & $<0.005$ & $<0.2$ & 19 & $<0.1$ & $<0.005$ & 24 & 280 & 8.7 \\
\hline East Transect Aguer & HG07-04/80-90 & 0.01 & 59 & 0.06 & $<0.005$ & $<0.2$ & 34 & $<0.1$ & $<0.005$ & 19 & 250 & 7.2 \\
\hline East Transect Aguer & HG07-04/90-95 & 0.04 & 61 & 0.50 & $<0.005$ & 0.23 & 42 & 0.2 & 0.006 & 20 & 270 & 9.1 \\
\hline East Transect Aguer & HG07-04/145-159 & $<0.01$ & 38 & 0.10 & $<0.005$ & $<0.2$ & 1.8 & $<0.1$ & $<0.005$ & 4.2 & 77 & 6.9 \\
\hline East Transect Aguer & HG07-04/159-163 & $<0.01$ & 30 & 0.10 & $<0.005$ & $<0.2$ & 2.2 & $<0.1$ & $<0.005$ & 3.1 & 55 & 9.5 \\
\hline East Transect Aguer & HG07-04/163-169 & $<0.01$ & 20 & 0.10 & $<0.005$ & $<0.2$ & 1.5 & $<0.1$ & $<0.005$ & 3.4 & 65 & 14 \\
\hline East Transect Aguer & HG07-04/169-177 & $<0.01$ & 21 & 0.04 & $<0.005$ & $<0.2$ & 1.4 & $<0.1$ & $<0.005$ & 4.1 & 65 & 15 \\
\hline East Transect Aguer & HG07-04/169-177d & $<0.01$ & 22 & 0.03 & $<0.005$ & $<0.2$ & 3.0 & $<0.1$ & $<0.005$ & 4.0 & 69 & 16 \\
\hline East Transect Aguer & HG07-04/177-184 & 0.03 & 54 & $<0.02$ & 0.006 & 0.23 & 81 & $<0.1$ & $<0.005$ & 2.6 & 22 & 14 \\
\hline East Transect Aguer & HG07-04/184-193 & 0.01 & 50 & $<0.02$ & $<0.005$ & $<0.2$ & 74 & $<0.1$ & $<0.005$ & 6.7 & 55 & 7.7 \\
\hline East Transect Aguer & HG07-04/184-193d & 0.01 & 50 & $<0.02$ & $<0.005$ & $<0.2$ & 65 & $<0.1$ & $<0.005$ & 6.5 & 50 & 7.6 \\
\hline East Transect Aguer & HG07-04/193-203 & 0.01 & 47 & $<0.02$ & $<0.005$ & 0.23 & 65 & $<0.1$ & $<0.005$ & 7.9 & 52 & 4.5 \\
\hline East Transect Aguer & HG07-04/203-217 & 0.02 & 38 & $<0.02$ & $<0.005$ & $<0.2$ & 61 & $<0.1$ & $<0.005$ & 8.4 & 49 & 8.9 \\
\hline East Transect Aguer & HG07-04/217-228 & 0.05 & 59 & $<0.02$ & 0.006 & $<0.2$ & 77 & $<0.1$ & $<0.005$ & 3.3 & 26 & 15 \\
\hline East Transect Aguer & HG07-04/217-228d & 0.03 & 47 & $<0.02$ & $<0.005$ & $<0.2$ & 63 & $<0.1$ & $<0.005$ & 4.6 & 44 & 24 \\
\hline East Transect Aguer & HG07-04/228-237 & 0.04 & 48 & $<0.02$ & 0.006 & $<0.2$ & 64 & $<0.1$ & $<0.005$ & 3.7 & 33 & 7.3 \\
\hline
\end{tabular}


Table 12A. Composition of the DI-leach bulk East transect auger hole samples. Method of determination (ICP-MS, inductively coupled plasma-mass spectrometry; ICP-OES, inductively coupled plasma-optical emission spectrometry; IC, ion chromatograph) is listed above each element.-Continued

[Sample site, name of sampling site; Sample ID, unique sample identifier; Treatment, type of sample preparation; d, duplicate; $\mu \mathrm{g} / \mathrm{L}, \mathrm{micrograms} \mathrm{per} \mathrm{liter;} \mathrm{mg/L,}$ milligrams per liter; $\mathrm{mg} / \mathrm{L} \mathrm{CaCO}_{3}$, milligrams per liter calcium carbonate]

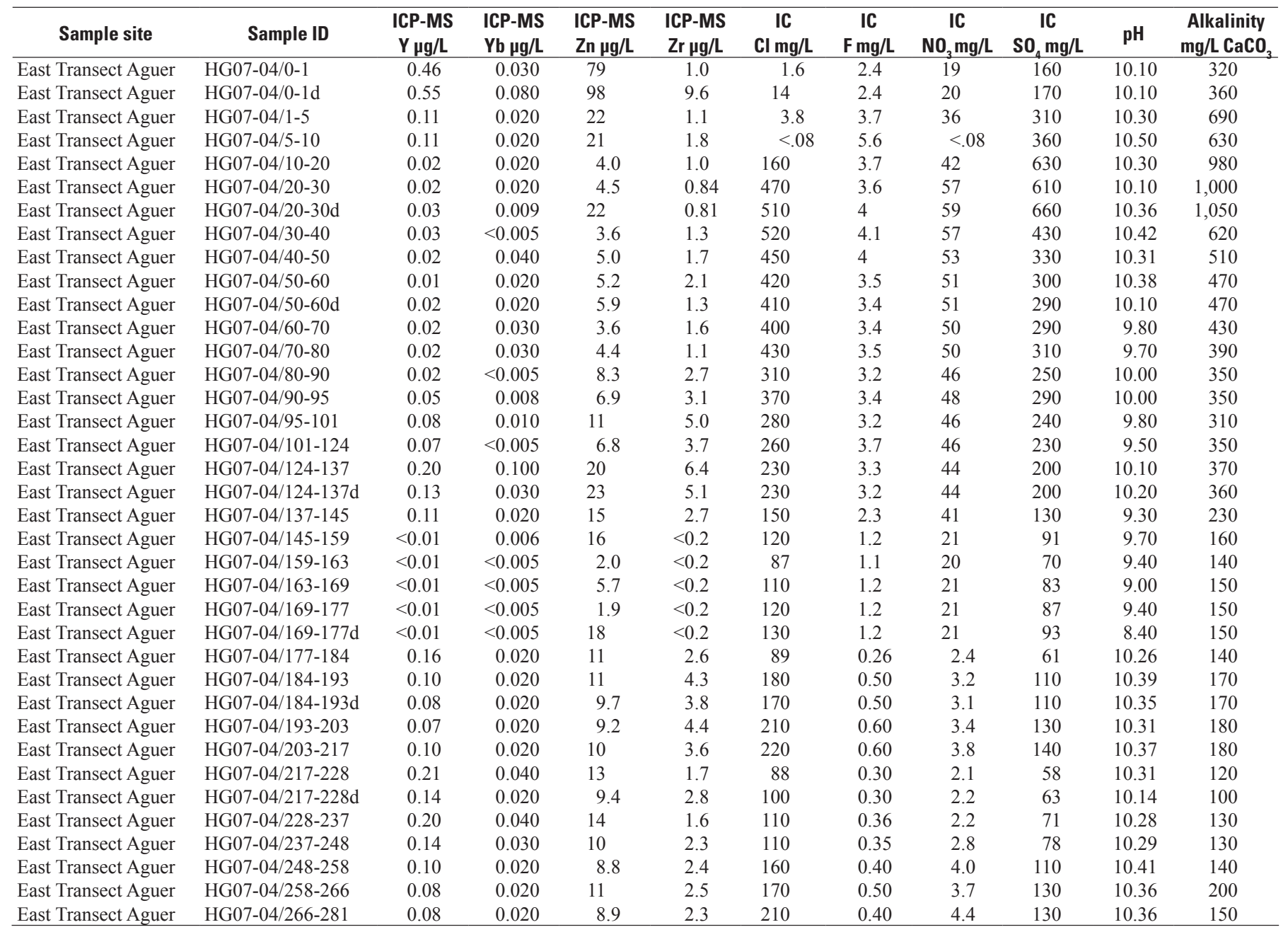


Table 12B. Composition of the nitric acid leach bulk East transect auger hole samples. Method of determination (ICP-MS, inductively coupled plasma-mass spectrometry; ICP-OES, inductively coupled plasma-optical emission spectrometry) is listed above each element.

[Sample site, name of sampling site; Sample ID, unique sample identifier; Treatment, type of sample preparation; col, DI-leach colloids; A, residue from Alkalinity measurement; $\mathrm{d}$, duplicate; $\mathrm{D}$, residue from DI leach; $\mu \mathrm{g} / \mathrm{L}$, micrograms per liter; $\mathrm{mg} / \mathrm{L}$, milligrams per liter]

\begin{tabular}{|c|c|c|c|c|c|c|c|c|c|c|c|}
\hline Sample site & Sample ID & Treatment & $\begin{array}{l}\text { ICP-MS } \\
\mathrm{Ag} \mu \mathrm{g} / \mathrm{L}\end{array}$ & $\begin{array}{c}\text { ICP-0ES } \\
\text { AI } \mu \mathrm{g} / \mathrm{L}\end{array}$ & $\begin{array}{l}\text { ICP-MS } \\
\text { As } \mu g / L\end{array}$ & $\begin{array}{l}\text { ICP-MS } \\
\text { Ba } \mu g / L\end{array}$ & $\begin{array}{c}\text { ICP-OES } \\
\text { B } \mu \mathrm{g} / \mathrm{L}\end{array}$ & $\begin{array}{l}\text { ICP-MS } \\
\text { Be } \mu \mathrm{g} / \mathrm{L}\end{array}$ & $\begin{array}{l}\text { ICP-MS } \\
\text { Bi } \mu \mathrm{g} / \mathrm{L}\end{array}$ & $\begin{array}{l}\text { ICP-MS } \\
\text { Ca mg/L }\end{array}$ & $\begin{array}{l}\text { ICP-MS } \\
\text { Cd } \mu \mathrm{g} / \mathrm{L}\end{array}$ \\
\hline East Transect Aguer & HG07-04/0-1A & Nitric Leach & $<3$ & 140,000 & 27 & 2,200 & 1,100 & 7.6 & 2.6 & 2,000 & 3.7 \\
\hline East Transect Aguer & HG07-04/0-1dA & Nitric Leach & $<3$ & 130,000 & 28 & 2,300 & 1,100 & 7.4 & 2.1 & 2,000 & 4.8 \\
\hline East Transect Aguer & HG07-04/0-1dD & Nitric Leach & $<3$ & 180,000 & 25 & 2,600 & 1,200 & 8.2 & $<2$ & 2,200 & 4.2 \\
\hline East Transect Aguer & HG07-04/1-5A & Nitric Leach & $<3$ & 150,000 & 25 & 2,600 & 1,400 & 7.6 & $<2$ & 2,200 & 4.4 \\
\hline East Transect Aguer & HG07-04/5-10A & Nitric Leach & $<3$ & 150,000 & 39 & 2,500 & 1,700 & 8.2 & $<2$ & 2,300 & 4.7 \\
\hline East Transect Aguer & HG07-04/20-30A & Nitric Leach & $<3$ & 120,000 & 95 & 2,200 & 2,800 & 7.7 & $<2$ & 2,400 & 4.9 \\
\hline East Transect Aguer & HG07-04/20-30dA & Nitric Leach & $<3$ & 110,000 & 91 & 2,200 & 2,900 & 7.0 & $<2$ & 2,300 & 4.5 \\
\hline East Transect Aguer & HG07-04/30-40A & Nitric Leach & $<3$ & 120,000 & 110 & 2,100 & 2,600 & 7.7 & $<2$ & 2,400 & 3.9 \\
\hline East Transect Aguer & HG07-04/40-50A & Nitric Leach & $<3$ & 95,000 & 87 & 1,400 & 1,500 & 6.3 & $<2$ & 1,400 & 2.4 \\
\hline East Transect Aguer & HG07-04/50-60A & Nitric Leach & $<3$ & 84,000 & 91 & 1,600 & 1,400 & 5.5 & $<2$ & 1,500 & 2.8 \\
\hline East Transect Aguer & HG07-04/50-60dA & Nitric Leach & $<3$ & 110,000 & 100 & 1,900 & 1,600 & 7.5 & $<2$ & 2,000 & 2.9 \\
\hline East Transect Aguer & HG07-04/101-124A & Nitric Leach & $<3$ & 98,000 & 83 & 1,800 & 1,800 & 7.8 & 2.8 & 2,300 & 3.9 \\
\hline East Transect Aguer & HG07-04/124-137A & Nitric Leach & $<3$ & 50,000 & 52 & 1,600 & 1,300 & 9.1 & $<2$ & 2,200 & 2.1 \\
\hline East Transect Aguer & HG0704/124-137dA & Nitric Leach & $<3$ & 46,000 & 45 & 1,500 & 1,100 & 4.9 & $<2$ & 2,100 & 2.7 \\
\hline East Transect Aguer & HG07-04/137-145A & Nitric Leach & $<3$ & 17,000 & 40 & 1,600 & 690 & 6.6 & $<2$ & 2,800 & 1.4 \\
\hline East Transect Aguer & HG07-04/137-145D & Nitric Leach & $<3$ & 11,000 & 26 & 1,200 & 380 & 1.3 & $<2$ & 2,200 & 0.83 \\
\hline East Transect Aguer & HG07-04/145-159A & Nitric Leach & $<3$ & 10,000 & 30 & 1,400 & 390 & 4.6 & $<2$ & 2,800 & 1.5 \\
\hline East Transect Aguer & HG07-04/159-163A & Nitric Leach & $<3$ & 8,800 & 32 & 1,500 & 270 & 1.9 & $<2$ & 2,700 & 1.6 \\
\hline East Transect Aguer & HG07-04/159-163D & Nitric Leach & $<3$ & 7,800 & 31 & 1,500 & 300 & 1.3 & $<2$ & 3,000 & 1.5 \\
\hline East Transect Aguer & HG07-04/163-169A & Nitric Leach & $<3$ & 10,000 & 27 & 1,500 & 390 & 1.9 & $<2$ & 2,900 & 1.7 \\
\hline East Transect Aguer & HG07-04/169-177A & Nitric Leach & $<3$ & 8,100 & 31 & 1,200 & 320 & 2.4 & $<2$ & 3,000 & 1.4 \\
\hline East Transect Aguer & HG07-04/169-177dA & Nitric Leach & $<3$ & 8,000 & 27 & 1,700 & 320 & 3.4 & $<2$ & 2,800 & 1.5 \\
\hline East Transect Aguer & HG07-04/177-184A & Nitric Leach & $<1$ & 8,000 & 29 & 1,300 & 360 & 2.4 & $<0.2$ & 3,000 & 1.1 \\
\hline East Transect Aguer & HG07-04/184-193A & Nitric Leach & $<1$ & 10,000 & 28 & 820 & 420 & 3.7 & $<0.2$ & 2,300 & 0.97 \\
\hline East Transect Aguer & HG07-04/237-248A & Nitric Leach & $<1$ & 6,600 & 39 & 1,200 & 300 & 2.9 & $<0.2$ & 3,100 & 1.6 \\
\hline East Transect Aguer & HG07-04/248-258 & Nitric Leach & $<1$ & 6,000 & 52 & 2,600 & 360 & 2.9 & $<0.2$ & 5,700 & 1.6 \\
\hline East Transect Aguer & HG07-04/258-266 & Nitric Leach & $<1$ & 16,000 & 39 & 1,200 & 420 & 3.0 & $<0.2$ & 2,600 & 1.3 \\
\hline East Transect Aguer & HG07-04/266-281 & Nitric Leach & $<1$ & 8,500 & 45 & 1,600 & 380 & 2.5 & $<0.2$ & 3,400 & 1.4 \\
\hline
\end{tabular}


Table 12B. Composition of the nitric acid leach bulk East transect auger hole samples. Method of determination (ICP-MS, inductively coupled plasma-mass spectrometry; ICP-OES, inductively coupled plasma-optical emission spectrometry) is listed above each element.-Continued

[Sample site, name of sampling site; Sample ID, unique sample identifier; Treatment, type of sample preparation; col, DI-leach colloids; A, residue from Alkalinity measurement; d, duplicate; D, residue from DI leach; $\mu \mathrm{g} / \mathrm{L}$, micrograms per liter; $\mathrm{mg} / \mathrm{L}$, milligrams per liter]

\begin{tabular}{|c|c|c|c|c|c|c|c|c|c|c|c|}
\hline Sample site & Sample ID & $\begin{array}{l}\text { ICP-MS } \\
\text { Ce } \mu g / L\end{array}$ & $\begin{array}{l}\text { ICP-MS } \\
\text { Co } \mu \mathrm{g} / \mathrm{L}\end{array}$ & $\begin{array}{l}\text { ICP-MS } \\
\text { Cr } \mu g / L\end{array}$ & $\begin{array}{l}\text { ICP-MS } \\
\text { Cs } \mu g / L\end{array}$ & $\begin{array}{l}\text { ICP-MS } \\
\text { Cu } \mu g / L\end{array}$ & $\begin{array}{l}\text { ICP-MS } \\
\text { Dy } \mu g / L\end{array}$ & $\begin{array}{l}\text { ICP-MS } \\
\text { Er } \mu \mathrm{g} / \mathrm{L}\end{array}$ & $\begin{array}{l}\text { ICP-MS } \\
\text { Eu } \mu g / L\end{array}$ & $\begin{array}{c}\text { ICP-OES } \\
\mathrm{Fe} \mu \mathrm{g} / \mathrm{L}\end{array}$ & $\begin{array}{l}\text { ICP-MS } \\
\text { Ga } \mu g / L\end{array}$ \\
\hline East Transect Aguer & HG07-04/0-1A & 670 & 14 & $<10$ & 0.49 & 39 & 45 & 24 & 9.8 & 6,400 & 12 \\
\hline East Transect Aguer & HG07-04/0-1dD & 720 & 19 & $<10$ & 0.37 & 43 & 46 & 25 & 9.6 & 6,000 & 12 \\
\hline East Transect Aguer & HG07-04/1-5A & 700 & 18 & $<10$ & 0.42 & 34 & 50 & 25 & 9.9 & 3,700 & 10 \\
\hline East Transect Aguer & HG07-04/5-10A & 820 & 21 & $<10$ & 0.36 & 31 & 51 & 28 & 11 & 3,600 & 11 \\
\hline East Transect Aguer & HG07-04/20-30A & 900 & 23 & $<10$ & 0.34 & 20 & 49 & 26 & 11 & 950 & 7.1 \\
\hline East Transect Aguer & HG07-04/20-30dA & 880 & 20 & $<10$ & 0.31 & 33 & 48 & 26 & 11 & 2,300 & 8.3 \\
\hline East Transect Aguer & HG07-04/30-40A & 870 & 23 & $<10$ & 0.40 & 29 & 51 & 27 & 10 & 1,700 & 7.5 \\
\hline East Transect Aguer & HG07-04/40-50A & 510 & 19 & $<10$ & 0.30 & 40 & 28 & 16 & 6.0 & 5,800 & 9.6 \\
\hline East Transect Aguer & HG07-04/50-60A & 530 & 19 & $<10$ & 0.41 & 39 & 31 & 16 & 6.5 & 7,000 & 10 \\
\hline East Transect Aguer & HG07-04/50-60dA & 580 & 21 & $<10$ & 0.31 & 40 & 34 & 18 & 7.2 & 8,100 & 12 \\
\hline East Transect Aguer & HG07-04/101-124A & 820 & 22 & $<10$ & 0.68 & 28 & 47 & 24 & 9.0 & 4,900 & 10 \\
\hline East Transect Aguer & HG07-04/124-137A & 620 & 14 & $<10$ & 0.85 & 28 & 35 & 20 & 5.4 & 7,300 & 8.7 \\
\hline East Transect Aguer & HG0704/124-137dA & 590 & 12 & $<10$ & 0.76 & 27 & 35 & 19 & 5.3 & 7,200 & 7.6 \\
\hline East Transect Aguer & HG07-04/137-145A & 550 & 6.2 & 11 & 0.56 & 21 & 35 & 19 & 4.4 & 3,900 & 5.4 \\
\hline East Transect Aguer & HG07-04/137-145D & 380 & 3.2 & $<10$ & 0.55 & 13 & 25 & 13 & 3.3 & 2,500 & 4.1 \\
\hline East Transect Aguer & HG07-04/145-159A & 490 & 4.4 & $<10$ & 0.67 & 19 & 31 & 17 & 4.1 & 3,200 & 5.1 \\
\hline East Transect Aguer & HG07-04/159-163A & 440 & 3.3 & 14 & 0.83 & 21 & 28 & 14 & 3.5 & 3,000 & 3.9 \\
\hline East Transect Aguer & HG07-04/159-163D & 450 & 6.1 & 15 & 0.71 & 15 & 28 & 15 & 3.5 & 2,200 & 3.6 \\
\hline East Transect Aguer & HG07-04/163-169A & 450 & 7.3 & 18 & 0.71 & 26 & 29 & 14 & 3.4 & 2,500 & 4.4 \\
\hline East Transect Aguer & HG07-04/169-177A & 430 & 9.1 & 16 & 0.77 & 22 & 28 & 15 & 3.5 & 2,400 & 3.3 \\
\hline East Transect Aguer & HG07-04/169-177dA & 430 & 9.1 & 13 & 0.96 & 27 & 27 & 15 & 3.7 & 3,000 & 3.9 \\
\hline East Transect Aguer & HG07-04/177-184A & 540 & 8.0 & 8.7 & 0.76 & 25 & 27 & 15 & 3.5 & 3,500 & 3.0 \\
\hline East Transect Aguer & HG07-04/184-193A & 530 & 11 & 6.6 & 1.2 & 22 & 26 & 14 & 3.5 & 4,500 & 3.5 \\
\hline East Transect Aguer & HG07-04/237-248A & 620 & 14 & 10 & 0.75 & 27 & 34 & 20 & 3.9 & 2,900 & 2.7 \\
\hline East Transect Aguer & HG07-04/248-258 & 740 & 29 & 15 & 0.97 & 37 & 37 & 22 & 4.9 & 3,100 & 3.6 \\
\hline East Transect Aguer & HG07-04/258-266 & 560 & 14 & 8.0 & 0.76 & 35 & 27 & 16 & 4.1 & 4,300 & 3.5 \\
\hline East Transect Aguer & HG07-04/266-281 & 590 & 8.8 & 11 & 0.70 & 34 & 30 & 17 & 4.2 & 3,200 & 2.8 \\
\hline
\end{tabular}


Table 12B. Composition of the nitric acid leach bulk East transect auger hole samples. Method of determination (ICP-MS, inductively coupled plasma-mass spectrometry; ICP-OES, inductively coupled plasma-optical emission spectrometry) is listed above each element.-Continued

[Sample site, name of sampling site; Sample ID, unique sample identifier; Treatment, type of sample preparation; col, DI-leach colloids; A, residue from Alkalinity measurement; d, duplicate; D, residue from DI leach; $\mu \mathrm{g} / \mathrm{L}$, micrograms per liter; $\mathrm{mg} / \mathrm{L}$, milligrams per liter]

\begin{tabular}{|c|c|c|c|c|c|c|c|c|c|c|c|c|}
\hline Sample site & Sample ID & $\begin{array}{l}\text { ICP-MS } \\
\text { Gd } \mu g / L\end{array}$ & $\begin{array}{l}\text { ICP-MS } \\
\text { Ge } \mu \mathrm{g} / \mathrm{L}\end{array}$ & $\begin{array}{l}\text { ICP-MS } \\
\text { Ho } \mu \mathrm{g} / \mathrm{L}\end{array}$ & $\begin{array}{c}\text { ICP-OES } \\
\mathrm{K} \mathrm{mg/L}\end{array}$ & $\begin{array}{l}\text { ICP-MS } \\
\text { La } \mu \mathrm{g} / \mathrm{L}\end{array}$ & $\begin{array}{c}\text { ICP-0ES } \\
\mathrm{Li} \mu \mathrm{g} / \mathrm{L}\end{array}$ & $\begin{array}{l}\text { ICP-MS } \\
\text { Lu } \mu g / L\end{array}$ & $\begin{array}{l}\text { ICP-OES } \\
\text { Mg mg/L }\end{array}$ & $\begin{array}{l}\text { ICP-MS } \\
\text { Mn } \mu g / L\end{array}$ & $\begin{array}{l}\text { ICP-MS } \\
\text { Mo } \mu \mathrm{g} / \mathrm{L}\end{array}$ & $\begin{array}{l}\text { ICP-OES } \\
\mathrm{Na} \mathrm{mg} / \mathrm{L}\end{array}$ \\
\hline East Transect Aguer & HG07-04/0-1A & 54 & 0.67 & 8.4 & 36 & 370 & 340 & 3 & 160 & 1,800 & $<20$ & 190 \\
\hline East Transect Aguer & HG07-04/0-1D & 57 & 1.4 & 8.8 & 38 & 380 & 480 & $>3$ & 190 & 2,200 & $<20$ & 220 \\
\hline East Transect Aguer & HG07-04/0-1dD & 57 & 1.9 & 9.1 & 39 & 390 & 510 & $>3$ & 200 & 2,200 & $<20$ & 220 \\
\hline East Transect Aguer & HG07-04/1-5A & 63 & 0.78 & 9.2 & $<10$ & 400 & 340 & $>3$ & 170 & 1,900 & $<20$ & 230 \\
\hline East Transect Aguer & HG07-04/5-10A & 62 & 0.88 & 9.7 & 46 & 420 & 360 & $>3$ & 180 & 2,100 & $<20$ & 240 \\
\hline East Transect Aguer & HG07-04/20-30A & 62 & 0.60 & 9.4 & 56 & 400 & 370 & $>3$ & 180 & 2,100 & $<20$ & 270 \\
\hline East Transect Aguer & HG07-04/20-30dA & 64 & 0.76 & 9.4 & 57 & 400 & 350 & $>3$ & 180 & 2,000 & $<20$ & 270 \\
\hline East Transect Aguer & HG07-04/30-40A & 60 & 1.1 & 10 & 58 & 410 & 280 & $>3$ & 160 & 2,200 & $<20$ & 240 \\
\hline East Transect Aguer & HG07-04/40-50A & 36 & 0.56 & 5.5 & 37 & 240 & 320 & 2.1 & 130 & 1,700 & $<20$ & 140 \\
\hline East Transect Aguer & HG07-04/50-60A & 41 & 0.53 & 6.0 & 42 & 260 & 280 & 2.2 & 120 & 1,700 & $<20$ & 140 \\
\hline East Transect Aguer & HG07-04/50-60dA & 44 & $<0.5$ & 6.8 & 50 & 280 & 300 & 2.4 & 140 & 1,900 & $<20$ & 170 \\
\hline East Transect Aguer & HG07-04/101-124A & 57 & 2.1 & 8.8 & 59 & 390 & 410 & 3 & 130 & 2,200 & $<20$ & 190 \\
\hline East Transect Aguer & HG07-04/124-137A & 46 & 2.6 & 7.6 & 54 & 340 & 540 & 2.6 & 120 & 1,600 & $<20$ & 170 \\
\hline East Transect Aguer & HG0704/124-137dA & 41 & 2.3 & 6.9 & 48 & 330 & 480 & 2.6 & 110 & 1,400 & $<20$ & 140 \\
\hline East Transect Aguer & HG07-04/137-145A & 42 & 2.2 & 6.5 & 29 & 330 & 230 & 2.5 & 70 & 1,300 & $<20$ & 98 \\
\hline East Transect Aguer & HG07-04/137-145D & 30 & 2.0 & 4.6 & 20 & 230 & 140 & 1.8 & 42 & 930 & $<20$ & 52 \\
\hline East Transect Aguer & HG07-04/145-159A & 37 & 2.1 & 6.3 & 26 & 290 & 120 & 2.2 & 55 & 1,300 & $<20$ & 46 \\
\hline East Transect Aguer & HG07-04/159-163A & 31 & 2.3 & 5.5 & 22 & 250 & 83 & 2.1 & 47 & 1,500 & $<20$ & 44 \\
\hline East Transect Aguer & HG07-04/159-163D & 32 & 1.3 & 5.4 & 20 & 250 & 77 & 2.2 & 46 & 1,400 & $<20$ & 33 \\
\hline East Transect Aguer & HG07-04/163-169A & 32 & 1.5 & 5.4 & 20 & 230 & 84 & 2.1 & 58 & 1,900 & $<20$ & 44 \\
\hline East Transect Aguer & HG07-04/169-177A & 31 & 1.6 & 5.2 & 21 & 240 & 84 & 2.1 & 56 & 1,700 & $<20$ & 44 \\
\hline East Transect Aguer & HG07-04/169-177dA & 32 & 1.3 & 5.5 & 23 & 240 & 100 & 1.9 & 50 & 1,800 & $<20$ & 37 \\
\hline East Transect Aguer & HG07-04/177-184A & 33 & 1.0 & 5.1 & 27 & 290 & 110 & 1.8 & 47 & 2,300 & $<2$ & 40 \\
\hline East Transect Aguer & HG07-04/184-193A & 33 & 1.2 & 5.0 & 47 & 290 & 110 & 1.8 & 44 & 2,300 & $<2$ & 60 \\
\hline East Transect Aguer & HG07-04/237-248A & 37 & 1.1 & 6.8 & 22 & 270 & 94 & 2.6 & 55 & 2,100 & $<2$ & 27 \\
\hline East Transect Aguer & HG07-04/248-258 & 44 & 1.4 & 7.3 & 21 & 370 & 97 & 2.7 & 62 & 3,800 & $<2$ & 31 \\
\hline East Transect Aguer & HG07-04/258-266 & 34 & 0.96 & 5.3 & 28 & 290 & 110 & 2.1 & 61 & 2,100 & $<2$ & 43 \\
\hline East Transect Aguer & HG07-04/266-281 & 36 & 0.93 & 5.7 & 23 & 330 & 70 & 2.2 & 64 & 2,200 & $<2$ & 34 \\
\hline
\end{tabular}


Table 12B. Composition of the nitric acid leach bulk East transect auger hole samples. Method of determination (ICP-MS, inductively coupled plasma-mass spectrometry; ICP-OES, inductively coupled plasma-optical emission spectrometry) is listed above each element.-Continued

[Sample site, name of sampling site; Sample ID, unique sample identifier; Treatment, type of sample preparation; col, DI-leach colloids; A, residue from Alkalinity measurement; d, duplicate; D, residue from DI leach; $\mu \mathrm{g} / \mathrm{L}$, micrograms per liter; $\mathrm{mg} / \mathrm{L}$, milligrams per liter]

\begin{tabular}{|c|c|c|c|c|c|c|c|c|c|c|c|c|}
\hline Sample site & Sample ID & $\begin{array}{l}\text { ICP-MS } \\
\mathrm{Nb} \mu \mathrm{g} / \mathrm{L}\end{array}$ & $\begin{array}{l}\text { ICP-MS } \\
\text { Nd } \mu \mathrm{g} / \mathrm{L}\end{array}$ & $\begin{array}{l}\text { ICP-MS } \\
\mathrm{Ni} \mu \mathrm{g} / \mathrm{L}\end{array}$ & $\begin{array}{c}\text { ICP-OES } \\
\text { P mg/L }\end{array}$ & $\begin{array}{l}\text { ICP-MS } \\
\mathrm{Pb} \mu \mathrm{g} / \mathrm{L}\end{array}$ & $\begin{array}{c}\text { ICP-MS } \\
\text { Pr } \mu g / L\end{array}$ & $\begin{array}{l}\text { ICP-MS } \\
\mathrm{Rb} \mu \mathrm{g} / \mathrm{L}\end{array}$ & $\begin{array}{l}\text { ICP-MS } \\
\text { Sb } \mu g / L\end{array}$ & $\begin{array}{l}\text { ICP-MS } \\
\text { Sc } \mu \mathrm{g} / \mathrm{L}\end{array}$ & $\begin{array}{l}\text { ICP-MS } \\
\text { Se } \mu g / L\end{array}$ & $\begin{array}{l}\text { ICP-OES } \\
\mathrm{SiO}_{2} \mathrm{mg} / \mathrm{L}\end{array}$ \\
\hline East Transect Aguer & HG07-04/0-1A & 16 & 320 & 24 & 7.1 & 140 & 86 & 29 & 17 & $<6$ & 11 & 55 \\
\hline East Transect Aguer & HG07-04/0-1D & 3.3 & 330 & 29 & 7.1 & 150 & 85 & 33 & $<3$ & 16 & 20 & 71 \\
\hline East Transect Aguer & HG07-04/0-1dD & 3.2 & 340 & 30 & 7.1 & 140 & 86 & 33 & $<3$ & 9.3 & 12 & 71 \\
\hline East Transect Aguer & HG07-04/1-5A & 12 & 350 & 25 & 7.5 & 150 & 94 & 30 & 12 & $<6$ & $<10$ & 56 \\
\hline East Transect Aguer & HG07-04/5-10A & 11 & 360 & 27 & 9.2 & 150 & 98 & 33 & 9.8 & $<6$ & 11 & 59 \\
\hline East Transect Aguer & HG07-04/20-30A & 8.7 & 350 & 26 & 10 & 100 & 95 & 33 & 6.8 & $<6$ & $<10$ & 57 \\
\hline East Transect Aguer & HG07-04/20-30dA & 7.8 & 350 & 23 & 11 & 120 & 91 & 36 & 6.2 & $<6$ & 17 & 56 \\
\hline East Transect Aguer & HG07-04/30-40A & 7.3 & 360 & 22 & 11 & 100 & 96 & 37 & 5.5 & $<6$ & 10 & 56 \\
\hline East Transect Aguer & HG07-04/40-50A & 7.1 & 210 & 21 & 7.4 & 100 & 54 & 33 & 4.9 & $<6$ & $<10$ & 64 \\
\hline East Transect Aguer & HG07-04/50-60A & 7.0 & 230 & 21 & 7.5 & 100 & 60 & 35 & 4.9 & $<6$ & $<10$ & 62 \\
\hline East Transect Aguer & HG07-04/50-60dA & 6.7 & 250 & 24 & 9.1 & 120 & 66 & 38 & 4.8 & $<6$ & 11 & 65 \\
\hline East Transect Aguer & HG07-04/95-101A & 16 & 340 & 28 & 9.7 & 72 & 89 & 39 & 22 & 7.3 & 16 & 51 \\
\hline East Transect Aguer & HG07-04/101-124A & 14 & 340 & 35 & 8.5 & 95 & 88 & 45 & 18 & 7.2 & $<10$ & 52 \\
\hline East Transect Aguer & HG07-04/124-137A & 10 & 280 & 26 & 5.1 & 81 & 77 & 45 & 12 & 7.0 & $<10$ & 49 \\
\hline East Transect Aguer & HG0704/124-137dA & 9.3 & 280 & 24 & 5.0 & 81 & 73 & 43 & 9.9 & $<6$ & 17 & 43 \\
\hline East Transect Aguer & HG07-04/137-145A & 7.6 & 270 & 15 & 3.3 & 67 & 73 & 28 & 7 & 6.6 & 28 & 27 \\
\hline East Transect Aguer & HG07-04/137-145D & 2.2 & 190 & 12 & 2.6 & 48 & 50 & 24 & $<3$ & $<6$ & 16 & 24 \\
\hline East Transect Aguer & HG07-04/145-159A & 6.6 & 240 & 14 & 2.8 & 65 & 64 & 23 & 6.1 & $<6$ & 11 & 18 \\
\hline East Transect Aguer & HG07-04/159-163A & 5.7 & 200 & 14 & 2.6 & 67 & 55 & 23 & 4.7 & 8.6 & 10 & 16 \\
\hline East Transect Aguer & HG07-04/159-163D & $<2$ & 200 & 9.1 & 2.7 & 62 & 55 & 21 & $<3$ & 6.6 & 16 & 15 \\
\hline East Transect Aguer & HG07-04/163-169A & 4.5 & 190 & 12 & 2.8 & 65 & 50 & 22 & 4.0 & 8.1 & $<10$ & 15 \\
\hline East Transect Aguer & HG07-04/169-177A & 4.4 & 200 & 17 & 2.6 & 63 & 54 & 25 & 3.4 & 8.3 & 16 & 18 \\
\hline East Transect Aguer & HG07-04/169-177dA & 3.6 & 200 & 17 & 2.6 & 64 & 53 & 28 & 3.6 & 8.0 & 25 & 20 \\
\hline East Transect Aguer & HG07-04/177-184A & $<0.2$ & 200 & $<0.4$ & 2.2 & 57 & 54 & 34 & $<0.3$ & 5.6 & 1.8 & 12 \\
\hline East Transect Aguer & HG07-04/237-248A & $<0.2$ & 200 & $<0.4$ & 1.9 & 58 & 52 & 34 & 0.31 & 5.4 & 1.9 & 12 \\
\hline East Transect Aguer & HG07-04/248-258 & $<0.2$ & 260 & $<0.4$ & 4.4 & 58 & 70 & 33 & 0.38 & 9.3 & 4.4 & 12 \\
\hline East Transect Aguer & HG07-04/258-266 & $<0.2$ & 210 & $<0.4$ & 2.3 & 61 & 56 & 35 & $<0.3$ & 6.6 & 2.0 & 12 \\
\hline East Transect Aguer & HG07-04/266-281 & $<0.2$ & 220 & $<0.4$ & 1.9 & 56 & 61 & 28 & $<0.3$ & 6.1 & 2.6 & 12 \\
\hline
\end{tabular}


Table 12B. Composition of the nitric acid leach bulk East transect auger hole samples. Method of determination (ICP-MS, inductively coupled plasma-mass spectrometry; ICP-OES, inductively coupled plasma-optical emission spectrometry) is listed above each element.—Continued

[Sample site, name of sampling site; Sample ID, unique sample identifier; Treatment, type of sample preparation; col, DI-leach colloids; A, residue from Alkalinity measurement; d, duplicate; D, residue from DI leach; $\mu \mathrm{g} / \mathrm{L}$, micrograms per liter; $\mathrm{mg} / \mathrm{L}$, milligrams per liter]

\begin{tabular}{|c|c|c|c|c|c|c|c|c|c|}
\hline Sample site & Sample ID & $\begin{array}{l}\text { ICP-MS } \\
\text { Sm } \mu \mathrm{g} / \mathrm{L}\end{array}$ & $\begin{array}{l}\text { ICP-MS } \\
\mathrm{SO}_{4} \mathrm{mg} / \mathrm{L}\end{array}$ & $\begin{array}{l}\text { ICP-MS } \\
\text { Sr } \mu g / L\end{array}$ & $\begin{array}{l}\text { ICP-MS } \\
\text { Ta } \mu \mathrm{g} / \mathrm{L}\end{array}$ & $\begin{array}{l}\text { ICP-MS } \\
\text { Tb } \mu g / L\end{array}$ & $\begin{array}{l}\text { ICP-MS } \\
\text { Th } \mu \mathrm{g} / \mathrm{L}\end{array}$ & $\begin{array}{c}\text { ICP-MS } \\
\mathrm{Ti} \mu \mathrm{g} / \mathrm{L}\end{array}$ & $\begin{array}{c}\text { ICP-MS } \\
\mathrm{TI} \mu \mathrm{g} / \mathrm{L}\end{array}$ \\
\hline East Transect Aguer & HG07-04/0-1A & 59 & $<20$ & 27,000 & 11 & 8.0 & 21 & 88 & 1.4 \\
\hline East Transect Aguer & HG07-04/0-1D & 62 & 55 & 27,000 & 2.5 & 8.5 & 23 & 68 & $<1$ \\
\hline East Transect Aguer & HG07-04/0-1dD & 65 & $<20$ & 28,000 & 1.5 & 8.7 & 19 & 37 & $<1$ \\
\hline East Transect Aguer & HG07-04/1-5A & 65 & 20 & 30,000 & 9.3 & 8.7 & 19 & 36 & 1.2 \\
\hline East Transect Aguer & HG07-04/5-10A & 72 & 37 & 32,000 & 8.7 & 8.8 & 19 & 38 & 1.2 \\
\hline East Transect Aguer & HG07-04/20-30A & 72 & 19 & 34,000 & 7.1 & 8.8 & 12 & 9.2 & 1.2 \\
\hline East Transect Aguer & HG07-04/20-30dA & 67 & $<20$ & 32,000 & 6.9 & 8.8 & 15 & 35 & 1.2 \\
\hline East Transect Aguer & HG07-04/30-40A & 72 & 36 & 32,000 & 6.6 & 9.3 & 13 & 18 & 1.2 \\
\hline East Transect Aguer & HG07-04/40-50A & 38 & 28 & 19,000 & 6.1 & 5.1 & 14 & 150 & $<1$ \\
\hline East Transect Aguer & HG07-04/50-60A & 43 & 32 & 18,000 & 5.9 & 5.8 & 15 & 190 & 1.0 \\
\hline East Transect Aguer & HG07-04/50-60dA & 48 & 33 & 24,000 & 6.2 & 6.7 & 21 & 170 & 1.2 \\
\hline East Transect Aguer & HG07-04/95-101A & 66 & $<20$ & 38,000 & 8.5 & 8.2 & 9 & 27 & 1.8 \\
\hline East Transect Aguer & HG07-04/101-124A & 64 & $<20$ & 26,000 & 7.9 & 8.4 & 17 & 71 & 1.5 \\
\hline East Transect Aguer & HG07-04/124-137A & 53 & $<20$ & 27,000 & 5.9 & 6.4 & 31 & 190 & 1.4 \\
\hline East Transect Aguer & HG0704/124-137dA & 50 & $<20$ & 25,000 & 5.2 & 6.4 & 33 & 200 & 1.2 \\
\hline East Transect Aguer & HG07-04/137-145A & 48 & $<20$ & 24,000 & 4.3 & 6.4 & 34 & 110 & 2.1 \\
\hline East Transect Aguer & HG07-04/137-145D & 34 & 56 & 16,000 & 1.4 & 4.1 & 23 & 120 & 1.5 \\
\hline East Transect Aguer & HG07-04/145-159A & 41 & 48 & 21,000 & 3.9 & 5.5 & 35 & 120 & 1.8 \\
\hline East Transect Aguer & HG07-04/159-163A & 38 & 74 & 20,000 & 3.4 & 4.9 & 32 & 110 & 2.2 \\
\hline East Transect Aguer & HG07-04/159-163D & 38 & 101 & 21,000 & 1.4 & 4.9 & 27 & 60 & 1.6 \\
\hline East Transect Aguer & HG07-04/163-169A & 35 & 70 & 21,000 & 2.7 & 4.7 & 32 & 68 & 1.9 \\
\hline East Transect Aguer & HG07-04/169-177A & 36 & 97 & 22,000 & 2.7 & 5.0 & 30 & 86 & 2.1 \\
\hline East Transect Aguer & HG07-04/169-177dA & 35 & 93 & 22,000 & 2.7 & 5.0 & 32 & 120 & 2.2 \\
\hline East Transect Aguer & HG07-04/177-184A & 36 & 5 & 21,000 & 0.03 & 4.8 & 42 & 190 & 2.2 \\
\hline East Transect Aguer & HG07-04/237-248A & 38 & 7 & 17,000 & 0.04 & 5.6 & 47 & 160 & 1.8 \\
\hline East Transect Aguer & HG07-04/248-258 & 49 & 23 & 21,000 & 0.05 & 6.5 & 44 & 200 & 1.8 \\
\hline East Transect Aguer & HG07-04/258-266 & 38 & 3 & 18,000 & 0.04 & 4.9 & 38 & 170 & 1.5 \\
\hline East Transect Aguer & HG07-04/266-281 & 41 & 6 & 19,000 & 0.04 & 5.3 & 42 & 130 & 1.6 \\
\hline
\end{tabular}


Table 12B. Composition of the nitric acid leach bulk East transect auger hole samples. Method of determination (ICP-MS, inductively coupled plasma-mass spectrometry; ICP-OES, inductively coupled plasma-optical emission spectrometry) is listed above each element.-Continued

[Sample site, name of sampling site; Sample ID, unique sample identifier; Treatment, type of sample preparation; col, DI-leach colloids; A, residue from Alkalinity measurement; d, duplicate; D, residue from DI leach; $\mu \mathrm{g} / \mathrm{L}$, micrograms per liter; $\mathrm{mg} / \mathrm{L}$, milligrams per liter]

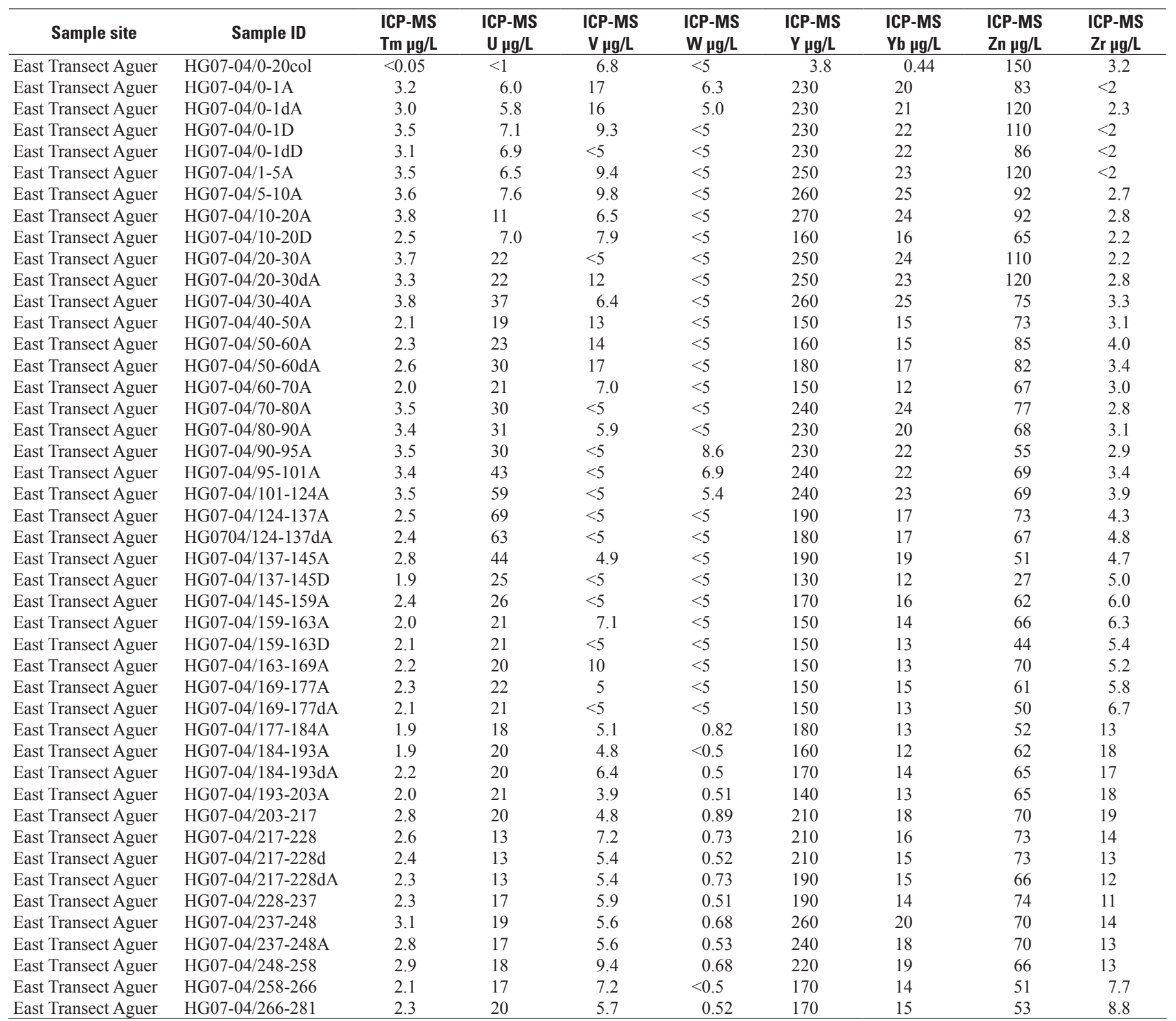


Table 12C. Composition of the residual fraction of bulk East transect auger hole samples. Method of determination (ICP-MS, inductively coupled plasma-mass spectrometry; ICP-OES, inductively coupled plasma-optical emission spectrometry) is listed above each element.

[Sample site, name of sampling site; Sample ID, unique sample identifier; Treatment, type of sample preparation; col, DI-leach colloids; A, residue from Alkalinity measurement; d, duplicate; D, residue from DI leach; ppm, parts per million; wt.\%, weight percent)

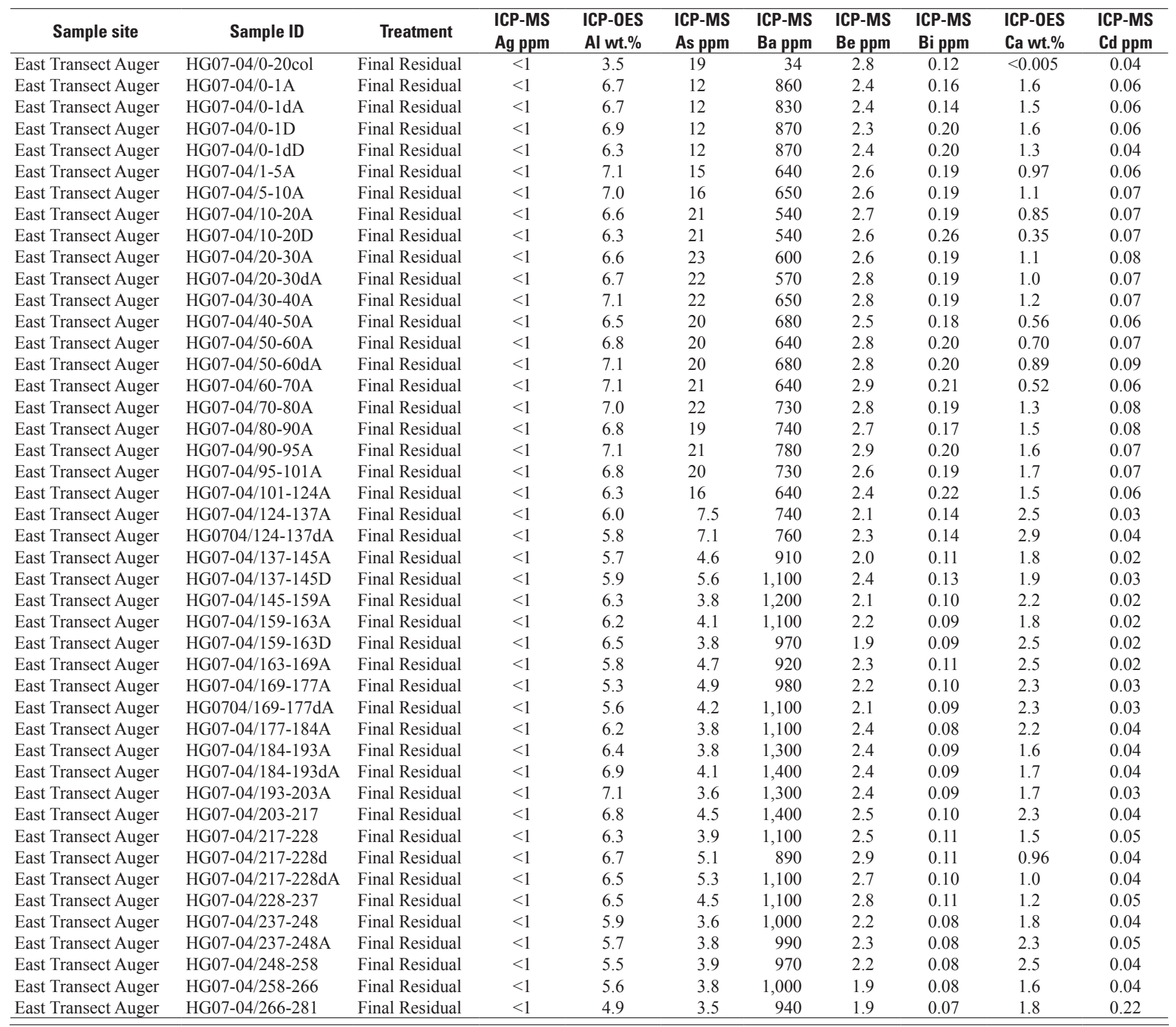


Table 12C. Composition of the residual fraction of bulk East transect auger hole samples. Method of determination (ICP-MS, inductively coupled plasma-mass spectrometry; ICP-OES, inductively coupled plasma-optical emission spectrometry) is listed above each element.-Continued

[Sample site, name of sampling site; Sample ID, unique sample identifier; Treatment, type of sample preparation; col, DI-leach colloids; A, residue from Alkalinity measurement; d, duplicate; D, residue from DI leach; ppm, parts per million; wt.\%, weight percent)

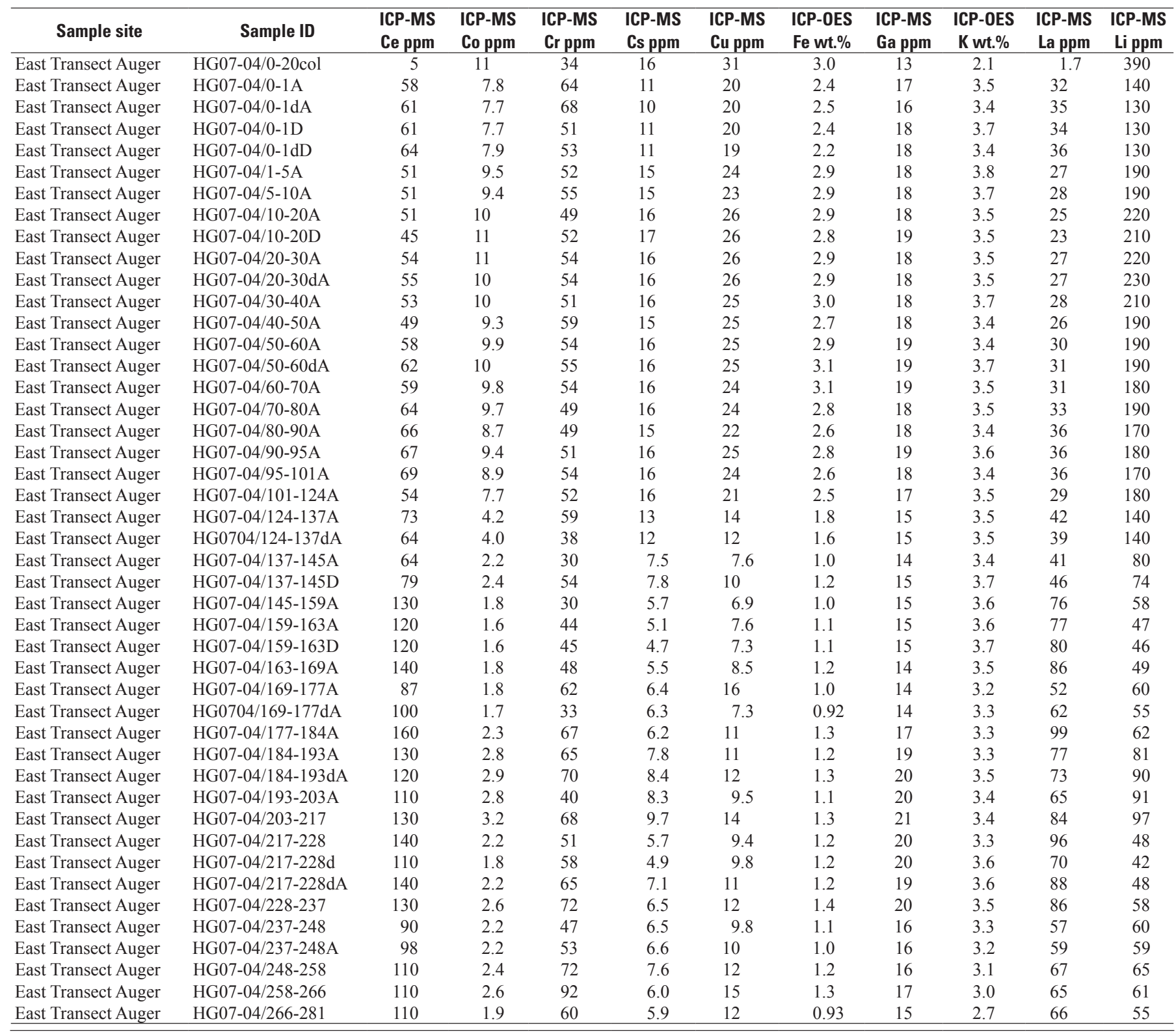


Table 12C. Composition of the residual fraction of bulk East transect auger hole samples. Method of determination (ICP-MS, inductively coupled plasma-mass spectrometry; ICP-OES, inductively coupled plasma-optical emission spectrometry) is listed above each element.-Continued

[Sample site, name of sampling site; Sample ID, unique sample identifier; Treatment, type of sample preparation; col, DI-leach colloids; A, residue from Alkalinity measurement; d, duplicate; D, residue from DI leach; ppm, parts per million; wt.\%, weight percent)

\begin{tabular}{|c|c|c|c|c|c|c|c|c|c|c|c|}
\hline Sample site & Sample ID & $\begin{array}{l}\text { ICP-0ES } \\
\text { Mg wt.\% }\end{array}$ & $\begin{array}{l}\text { ICP-MS } \\
\text { Mn ppm }\end{array}$ & $\begin{array}{l}\text { ICP-MS } \\
\text { Mo ppm }\end{array}$ & $\begin{array}{l}\text { ICP-0ES } \\
\text { Na wt.\% }\end{array}$ & $\begin{array}{l}\text { ICP-MS } \\
\text { Nb ppm }\end{array}$ & $\begin{array}{l}\text { ICP-OES } \\
\text { Nd ppm }\end{array}$ & $\begin{array}{l}\text { ICP-MS } \\
\text { Ni ppm }\end{array}$ & $\begin{array}{c}\text { ICP-MS } \\
\text { P ppm }\end{array}$ & $\begin{array}{l}\text { ICP-MS } \\
\text { Pb ppm }\end{array}$ & $\begin{array}{l}\text { ICP-MS } \\
\text { Rb ppm }\end{array}$ \\
\hline East Transect Auger & HG07-04/0-1A & 2.8 & 550 & 1.0 & 1.7 & 23 & 17 & 17 & 280 & 17 & 150 \\
\hline East Transect Auger & HG07-04/0-1dD & 2.6 & 550 & 0.9 & 1.6 & 25 & 19 & 16 & 290 & 17 & 160 \\
\hline East Transect Auger & HG07-04/1-5A & 3.9 & 660 & 0.9 & 1.5 & 25 & 14 & 20 & 290 & 16 & 170 \\
\hline East Transect Auger & HG07-04/5-10A & 3.8 & 650 & 0.8 & 1.4 & 24 & 16 & 20 & 290 & 16 & 170 \\
\hline East Transect Auger & HG07-04/20-30A & 4.5 & 720 & 0.9 & 1.2 & 24 & 16 & 22 & 350 & 18 & 170 \\
\hline East Transect Auger & HG07-04/20-30dA & 4.7 & 720 & 0.8 & 1.2 & 23 & 15 & 22 & 330 & 17 & 170 \\
\hline East Transect Auger & HG07-04/30-40A & 4.5 & 690 & 0.9 & 1.3 & 24 & 17 & 21 & 340 & 18 & 170 \\
\hline East Transect Auger & HG07-04/40-50A & 3.8 & 630 & 0.9 & 1.1 & 24 & 13 & 20 & 230 & 15 & 170 \\
\hline East Transect Auger & HG07-04/50-60A & 3.8 & 660 & 0.9 & 1.1 & 26 & 17 & 21 & 250 & 16 & 170 \\
\hline East Transect Auger & HG07-04/50-60dA & 3.8 & 690 & 0.9 & 1.2 & 27 & 17 & 21 & 280 & 17 & 180 \\
\hline East Transect Auger & HG07-04/101-124A & 3.6 & 550 & 0.9 & 1.4 & 26 & 17 & 16 & 220 & 17 & 160 \\
\hline East Transect Auger & HG07-04/124-137A & 2.8 & 390 & 0.9 & 1.8 & 26 & 21 & 9.9 & 150 & 14 & 130 \\
\hline East Transect Auger & HG0704/124-137dA & 2.7 & 380 & 0.7 & 1.8 & 26 & 21 & 8.5 & 180 & 15 & 140 \\
\hline East Transect Auger & HG07-04/137-145A & 1.5 & 260 & 0.7 & 2.1 & 24 & 19 & 5.0 & 140 & 14 & 110 \\
\hline East Transect Auger & HG07-04/137-145D & 1.3 & 270 & 0.9 & 2.1 & 28 & 24 & 6.4 & 150 & 16 & 130 \\
\hline East Transect Auger & HG07-04/145-159A & 0.98 & 250 & 0.6 & 2.4 & 33 & 37 & 4.3 & 180 & 17 & 110 \\
\hline East Transect Auger & HG07-04/159-163A & 0.73 & 220 & 0.9 & 2.4 & 26 & 34 & 4.6 & 150 & 14 & 110 \\
\hline East Transect Auger & HG07-04/159-163D & 0.76 & 210 & 0.8 & 2.5 & 27 & 38 & 4.5 & 170 & 14 & 100 \\
\hline East Transect Auger & HG07-04/163-169A & 0.85 & 260 & 0.9 & 2.2 & 31 & 41 & 4.9 & 170 & 15 & 110 \\
\hline East Transect Auger & HG07-04/169-177A & 0.86 & 230 & 0.9 & 2.0 & 25 & 24 & 5.7 & 140 & 16 & 110 \\
\hline East Transect Auger & HG0704/169-177dA & 0.93 & 220 & 0.7 & 2.1 & 28 & 29 & 4.4 & 190 & 14 & 110 \\
\hline East Transect Auger & HG07-04/177-184A & 0.94 & 310 & 1.2 & 2.2 & 38 & 39 & 6.1 & 170 & 16 & 120 \\
\hline East Transect Auger & HG07-04/184-193A & 1.1 & 300 & 1.2 & 2.1 & 34 & 31 & 7.3 & 160 & 18 & 140 \\
\hline East Transect Auger & HG07-04/237-248A & 0.83 & 220 & 0.9 & 1.9 & 31 & 24 & 6.1 & 170 & 17 & 130 \\
\hline East Transect Auger & HG07-04/248-258 & 0.98 & 260 & 1.2 & 1.7 & 32 & 26 & 7.4 & 170 & 16 & 130 \\
\hline East Transect Auger & HG07-04/258-266 & 0.82 & 260 & 1.4 & 1.8 & 30 & 24 & 8.8 & 150 & 18 & 120 \\
\hline East Transect Auger & HG07-04/266-281 & 0.69 & 220 & 1.1 & 1.6 & 28 & 25 & 6.3 & 150 & 15 & 110 \\
\hline
\end{tabular}


Table 12C. Composition of the residual fraction of bulk East transect auger hole samples. Method of determination (ICP-MS, inductively coupled plasma-mass spectrometry; ICP-OES, inductively coupled plasma-optical emission spectrometry) is listed above each element.-Continued

[Sample site, name of sampling site; Sample ID, unique sample identifier; Treatment, type of sample preparation; col, DI-leach colloids; A, residue from Alkalinity measurement; d, duplicate; D, residue from DI leach; ppm, parts per million; wt.\%, weight percent)

\begin{tabular}{|c|c|c|c|c|c|c|c|c|c|c|c|}
\hline Sample site & Sample ID & $\begin{array}{l}\text { ICP-MS } \\
\text { Sb ppm }\end{array}$ & $\begin{array}{l}\text { ICP-MS } \\
\text { Sc ppm }\end{array}$ & $\begin{array}{l}\text { ICP-MS } \\
\text { Sr ppm }\end{array}$ & $\begin{array}{l}\text { ICP-MS } \\
\text { Th ppm }\end{array}$ & $\begin{array}{l}\text { ICP-MS } \\
\text { Ti ppm }\end{array}$ & $\begin{array}{c}\text { ICP-MS } \\
\text { TI ppm }\end{array}$ & $\begin{array}{l}\text { ICP-MS } \\
\text { U ppm }\end{array}$ & $\begin{array}{c}\text { ICP-MS } \\
\text { V ppm }\end{array}$ & $\begin{array}{c}\text { ICP-MS } \\
\text { Y ppm }\end{array}$ & $\begin{array}{l}\text { ICP-MS } \\
\text { Zn ppm }\end{array}$ \\
\hline East Transect Auger & HG07-04/0-1A & 1.3 & 7.8 & 300 & 12 & 2,700 & 0.9 & 2.2 & 50 & 13 & 70 \\
\hline East Transect Auger & HG07-04/0-1dD & 1.3 & 8.3 & 310 & 14 & 2,800 & 1.0 & 2.2 & 50 & 14 & 72 \\
\hline East Transect Auger & HG07-04/1-5A & 1.6 & 9.9 & 220 & 14 & 2,900 & 1.1 & 2.4 & 57 & 13 & 85 \\
\hline East Transect Auger & HG07-04/5-10A & 1.6 & 9.8 & 230 & 14 & 2,900 & 1.1 & 2.3 & 57 & 12 & 84 \\
\hline East Transect Auger & HG07-04/20-30A & 1.7 & 10 & 230 & 15 & 2,800 & 1.1 & 2.6 & 57 & 12 & 92 \\
\hline East Transect Auger & HG07-04/20-30dA & 1.6 & 10 & 210 & 15 & 2,900 & 1.1 & 2.5 & 56 & 12 & 90 \\
\hline East Transect Auger & HG07-04/30-40A & 1.6 & 10 & 250 & 15 & 2,800 & 1.2 & 2.6 & 57 & 12 & 91 \\
\hline East Transect Auger & HG07-04/40-50A & 1.5 & 9.9 & 190 & 14 & 2,800 & 1.1 & 2.4 & 57 & 11 & 86 \\
\hline East Transect Auger & HG07-04/50-60A & 1.6 & 10 & 200 & 15 & 3,100 & 1.2 & 2.7 & 59 & 12 & 92 \\
\hline East Transect Auger & HG07-04/50-60dA & 1.6 & 10 & 220 & 16 & 3,000 & 1.2 & 2.5 & 60 & 14 & 91 \\
\hline East Transect Auger & HG07-04/101-124A & 1.4 & 8.1 & 280 & 15 & 2,500 & 1.1 & 2.7 & 44 & 13 & 77 \\
\hline East Transect Auger & HG07-04/124-137A & 1.2 & 5.0 & 330 & 13 & 1,900 & 1.0 & 2.8 & 24 & 14 & 50 \\
\hline East Transect Auger & HG0704/124-137dA & 1.2 & 5.0 & 390 & 13 & 1,800 & 1.0 & 2.7 & 24 & 15 & 50 \\
\hline East Transect Auger & HG07-04/137-145A & 0.7 & 3.2 & 320 & 12 & 1,400 & 1.0 & 2.3 & 12 & 16 & 32 \\
\hline East Transect Auger & HG07-04/137-145D & 0.9 & 3.4 & 290 & 14 & 1,400 & 1.0 & 2.8 & 14 & 17 & 35 \\
\hline East Transect Auger & HG07-04/145-159A & 0.7 & 3.5 & 360 & 15 & 1,800 & 0.9 & 2.4 & 12 & 24 & 32 \\
\hline East Transect Auger & HG07-04/159-163A & 0.6 & 2.8 & 320 & 14 & 1,500 & 0.8 & 2.1 & 11 & 22 & 27 \\
\hline East Transect Auger & HG07-04/159-163D & 0.6 & 3.0 & 340 & 14 & 1,500 & 0.8 & 2.0 & 10 & 19 & 27 \\
\hline East Transect Auger & HG07-04/163-169A & 0.7 & 3.1 & 310 & 16 & 1,800 & 1.0 & 2.4 & 12 & 24 & 32 \\
\hline East Transect Auger & HG07-04/169-177A & 0.8 & 2.7 & 330 & 13 & 1,300 & 0.9 & 2.3 & 11 & 17 & 30 \\
\hline East Transect Auger & HG0704/169-177dA & 0.7 & 2.8 & 350 & 14 & 1,300 & 0.9 & 2.2 & 11 & 16 & 29 \\
\hline East Transect Auger & HG07-04/177-184A & 0.8 & 3.7 & 320 & 17 & 2,100 & 1.0 & 2.5 & 14 & 22 & 38 \\
\hline East Transect Auger & HG07-04/184-193A & 0.9 & 3.8 & 410 & 16 & 1,800 & 1.0 & 2.5 & 15 & 20 & 38 \\
\hline East Transect Auger & HG07-04/237-248A & 0.7 & 2.9 & 330 & 14 & 1,300 & 1.0 & 2.3 & 16 & 18 & 34 \\
\hline East Transect Auger & HG07-04/248-258 & 0.8 & 3.3 & 350 & 15 & 1,600 & 1.0 & 2.4 & 17 & 20 & 37 \\
\hline East Transect Auger & HG07-04/258-266 & 0.8 & 3.3 & 330 & 13 & 1,900 & 0.9 & 2.0 & 18 & 18 & 37 \\
\hline East Transect Auger & HG07-04/266-281 & 0.7 & 3.0 & 320 & 13 & 1,500 & 0.9 & 2.3 & 14 & 18 & 32 \\
\hline
\end{tabular}


Table 12D. Composition of the DI-leach bulk grab samples. Method of determination (ICP-MS, inductively coupled plasmamass spectrometry; ICP-OES, inductively coupled plasma-optical emission spectrometry; IC, ion chromatograph) is listed above each element.

[Leach batch, denotes the batch in which the sample was processed and analyzed; Sample site, name of sampling site; Sample ID, unique sample identifier; -1, -2, denotes duplicates; BC, bag contents-hydrated salts converted to liquid upon return from field; d, duplicate; n.d., not determined; ins, insufficient sample; $\mu \mathrm{g} / \mathrm{L}$, micrograms per liter; $\mathrm{mg} / \mathrm{L}$, milligrams per liter; $\mathrm{mg} / \mathrm{L} \mathrm{CaCO}_{3}$, milligrams per liter calcium carbonate]

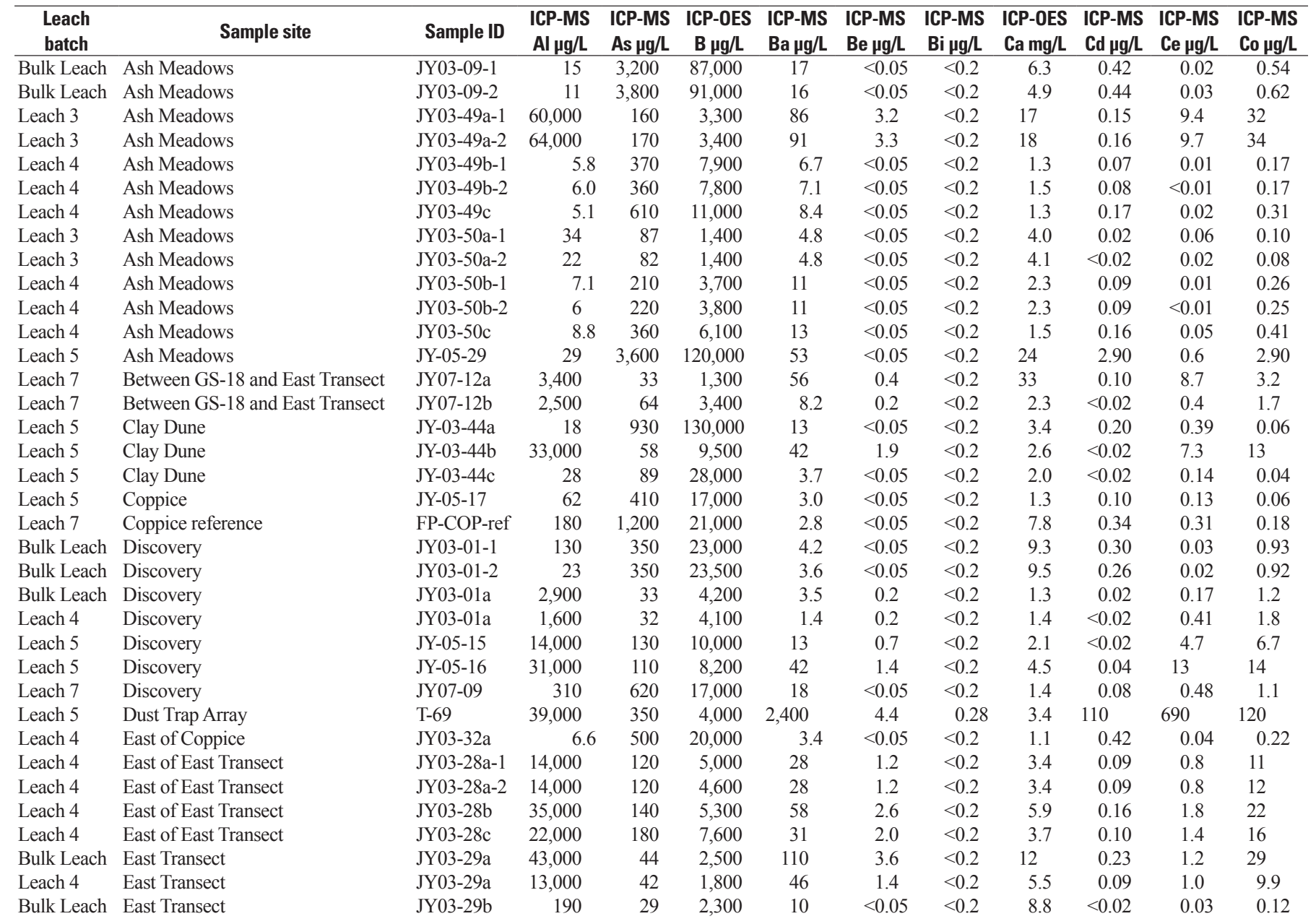


Table 12D. Composition of the DI-leach bulk grab samples. Method of determination (ICP-MS, inductively coupled plasmamass spectrometry; ICP-0ES, inductively coupled plasma-optical emission spectrometry; IC, ion chromatograph) is listed above each element.-Continued

[Leach batch, denotes the batch in which the sample was processed and analyzed; Sample site, name of sampling site; Sample ID, unique sample identifier; $-1,-2$, denotes duplicates; BC, bag contents-hydrated salts converted to liquid upon return from field; d, duplicate; n.d., not determined; ins, insufficient sample; $\mu \mathrm{g} / \mathrm{L}$, micrograms per liter; $\mathrm{mg} / \mathrm{L}$, milligrams per liter; $\mathrm{mg} / \mathrm{L} \mathrm{CaCO}$, milligrams per liter calcium carbonate]

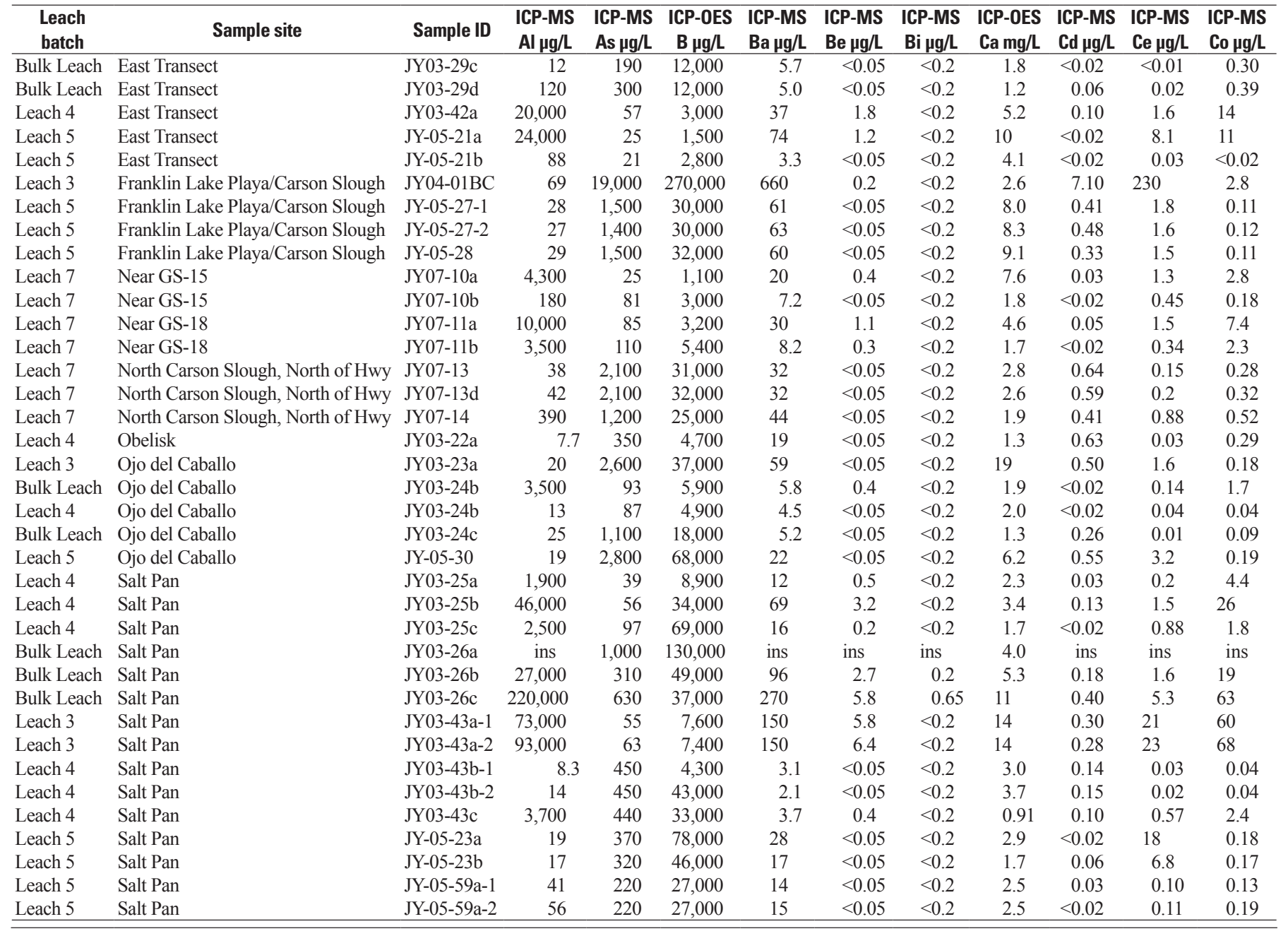


Table 12D. Composition of the DI-leach bulk grab samples. Method of determination (ICP-MS, inductively coupled plasmamass spectrometry; ICP-OES, inductively coupled plasma-optical emission spectrometry; IC, ion chromatograph) is listed above each element.-Continued

[Leach batch, denotes the batch in which the sample was processed and analyzed; Sample site, name of sampling site; Sample ID, unique sample identifier; $-1,-2$, denotes duplicates; $\mathrm{BC}$, bag contents-hydrated salts converted to liquid upon return from field; d, duplicate; n.d., not determined; ins, insufficient sample; $\mu \mathrm{g} / \mathrm{L}$, micrograms per liter; $\mathrm{mg} / \mathrm{L}$, milligrams per liter; $\mathrm{mg} / \mathrm{L} \mathrm{CaCO}_{3}$, milligrams per liter calcium carbonate]

\begin{tabular}{|c|c|c|c|c|c|c|c|c|c|c|c|c|}
\hline $\begin{array}{l}\text { Leach } \\
\text { batch }\end{array}$ & Sample site & Sample ID & $\begin{array}{c}\text { ICP-MS } \\
\text { Cr } \mu g / L\end{array}$ & $\begin{array}{l}\text { ICP-MS } \\
\text { Cs } \mu g / L\end{array}$ & $\begin{array}{l}\text { ICP-MS } \\
\text { Cu } \mu g / L\end{array}$ & $\begin{array}{l}\text { ICP-MS } \\
\text { Dy } \mu \mathrm{g} / \mathrm{L}\end{array}$ & $\begin{array}{c}\text { ICP-MS } \\
\text { Er } \mu \mathrm{g} / \mathrm{L}\end{array}$ & $\begin{array}{l}\text { ICP-MS } \\
\text { Eu } \mu g / L\end{array}$ & $\begin{array}{c}\text { ICP-OES } \\
\mathrm{Fe} \mu \mathrm{g} / \mathrm{L}\end{array}$ & $\begin{array}{l}\text { ICP-MS } \\
\text { Ga } \mu g / L\end{array}$ & $\begin{array}{l}\text { ICP-MS } \\
\text { Gd } \mu \mathrm{g} / \mathrm{L}\end{array}$ & $\begin{array}{l}\text { ICP-MS } \\
\text { Ge } \mu \mathrm{g} / \mathrm{L}\end{array}$ \\
\hline Bulk Leach & Ash Meadows & JY03-09-2 & $<1$ & 0.21 & 9.6 & $<0.005$ & $<0.005$ & $<0.005$ & $<20$ & 0.08 & $<0.005$ & 0.10 \\
\hline Leach 3 & Ash Meadows & JY03-49a-1 & 32 & 9.4 & 63 & 0.57 & 0.29 & 0.13 & 34,000 & 18 & 0.75 & 2.1 \\
\hline Leach 4 & Ash Meadows & JY03-49b-1 & 1.9 & 0.12 & 4.2 & n.d. & n.d. & n.d. & $<20$ & $<0.05$ & n.d. & 0.08 \\
\hline Leach 4 & Ash Meadows & JY03-49b-2 & 1.8 & 0.13 & 5.4 & n.d. & n.d. & n.d. & $<20$ & $<0.05$ & n.d. & 0.06 \\
\hline Leach 4 & Ash Meadows & JY03-49c & 2.7 & 0.15 & 4.0 & n.d. & n.d. & n.d. & $<20$ & $<0.05$ & n.d. & $<0.05$ \\
\hline Leach 4 & Ash Meadows & JY03-50b-1 & $<1$ & 0.13 & 8.4 & n.d. & n.d. & n.d. & $<20$ & $<0.05$ & n.d. & 0.07 \\
\hline Leach 4 & Ash Meadows & JY03-50b-2 & $<1$ & 0.14 & 8.4 & n.d. & n.d. & n.d. & $<20$ & $<0.05$ & n.d. & 0.08 \\
\hline Leach 4 & Ash Meadows & JY03-50c & $<1$ & 0.14 & 5.2 & n.d. & n.d. & n.d. & $<20$ & $<0.05$ & n.d. & $<0.05$ \\
\hline Leach 5 & Ash Meadows & JY-05-29 & 2.0 & 0.28 & 22 & 0.08 & 0.11 & $<0.005$ & $<20$ & $<0.05$ & 0.01 & $<0.05$ \\
\hline Leach 7 & Between GS-18 and East Transect & JY07-12a & 2.6 & 0.12 & 20 & 0.53 & 0.3 & 0.11 & 3,100 & 2.0 & 0.68 & 0.65 \\
\hline Leach 7 & Between GS-18 and East Transect & JY07-12b & 4.1 & 0.48 & 7.3 & 0.02 & 0.01 & $<0.005$ & 2,100 & 1.2 & 0.02 & 0.43 \\
\hline Bulk Leach & Discovery & JY03-01-1 & 1.6 & 0.12 & 9.3 & $<0.005$ & $<0.005$ & $<0.005$ & 77 & 0.10 & $<0.005$ & $<0.05$ \\
\hline Bulk Leach & Discovery & JY03-01-2 & 1.5 & 0.05 & 6.5 & $<0.005$ & $<0.005$ & $<0.005$ & $<20$ & 0.07 & $<0.005$ & $<0.05$ \\
\hline Bulk Leach & Discovery & JY03-01a & 2.6 & 0.92 & 6.9 & 0.008 & 0.005 & $<0.005$ & 1,600 & 0.96 & 0.01 & 0.20 \\
\hline Leach 4 & Discovery & JY03-01a & 1.5 & 0.36 & 6.6 & n.d. & n.d. & n.d. & 1,300 & 0.66 & n.d. & 0.25 \\
\hline Leach 5 & Discovery & JY-05-15 & 12 & 3.9 & 25 & 0.22 & 0.14 & 0.06 & 15,000 & 4.8 & 0.29 & 1.1 \\
\hline Leach 5 & Discovery & JY-05-16 & 15 & 8.0 & 38 & 0.58 & 0.36 & 0.12 & 35,000 & 9.6 & 0.80 & 1.7 \\
\hline Leach 7 & Discovery & JY07-09 & 1.3 & 0.13 & 24 & 0.02 & 0.02 & 0.01 & 320 & 0.20 & 0.04 & 0.10 \\
\hline Leach 5 & Dust Trap Array & $\mathrm{T}-69$ & 72 & 0.19 & 2,600 & 42 & 23 & 9.1 & 3,700 & 6.2 & 57 & 1.3 \\
\hline Leach 4 & East of Coppice & JY03-32a & $<1$ & 0.05 & 4.6 & n.d. & n.d. & n.d. & $<20$ & 0.07 & n.d. & $<0.05$ \\
\hline Leach 4 & East of East Transect & JY03-28a-1 & 12 & 3.5 & 28 & n.d. & n.d. & n.d. & 11,000 & 6.8 & n.d. & 2.0 \\
\hline Leach 4 & East of East Transect & JY03-28a-2 & 12 & 3.8 & 26 & n.d. & n.d. & n.d. & 11,000 & 7.0 & n.d. & 2.0 \\
\hline Leach 4 & East of East Transect & JY03-28b & 25 & 12 & 54 & n.d. & n.d. & n.d. & 20,000 & 17 & n.d. & 6.7 \\
\hline Leach 4 & East of East Transect & JY03-28c & 15 & 6.7 & 37 & n.d. & n.d. & n.d. & 7,000 & 11 & n.d. & 3.1 \\
\hline
\end{tabular}


Table 12D. Composition of the DI-leach bulk grab samples. Method of determination (ICP-MS, inductively coupled plasmamass spectrometry; ICP-OES, inductively coupled plasma-optical emission spectrometry; IC, ion chromatograph) is listed above each element.-Continued

[Leach batch, denotes the batch in which the sample was processed and analyzed; Sample site, name of sampling site; Sample ID, unique sample identifier; $-1,-2$, denotes duplicates; $\mathrm{BC}$, bag contents-hydrated salts converted to liquid upon return from field; $d$, duplicate; n.d., not determined; ins, insufficient sample; $\mu \mathrm{g} / \mathrm{L}$, micrograms per liter; $\mathrm{mg} / \mathrm{L}$, milligrams per liter; $\mathrm{mg} / \mathrm{L} \mathrm{CaCO}_{3}$, milligrams per liter calcium carbonate]

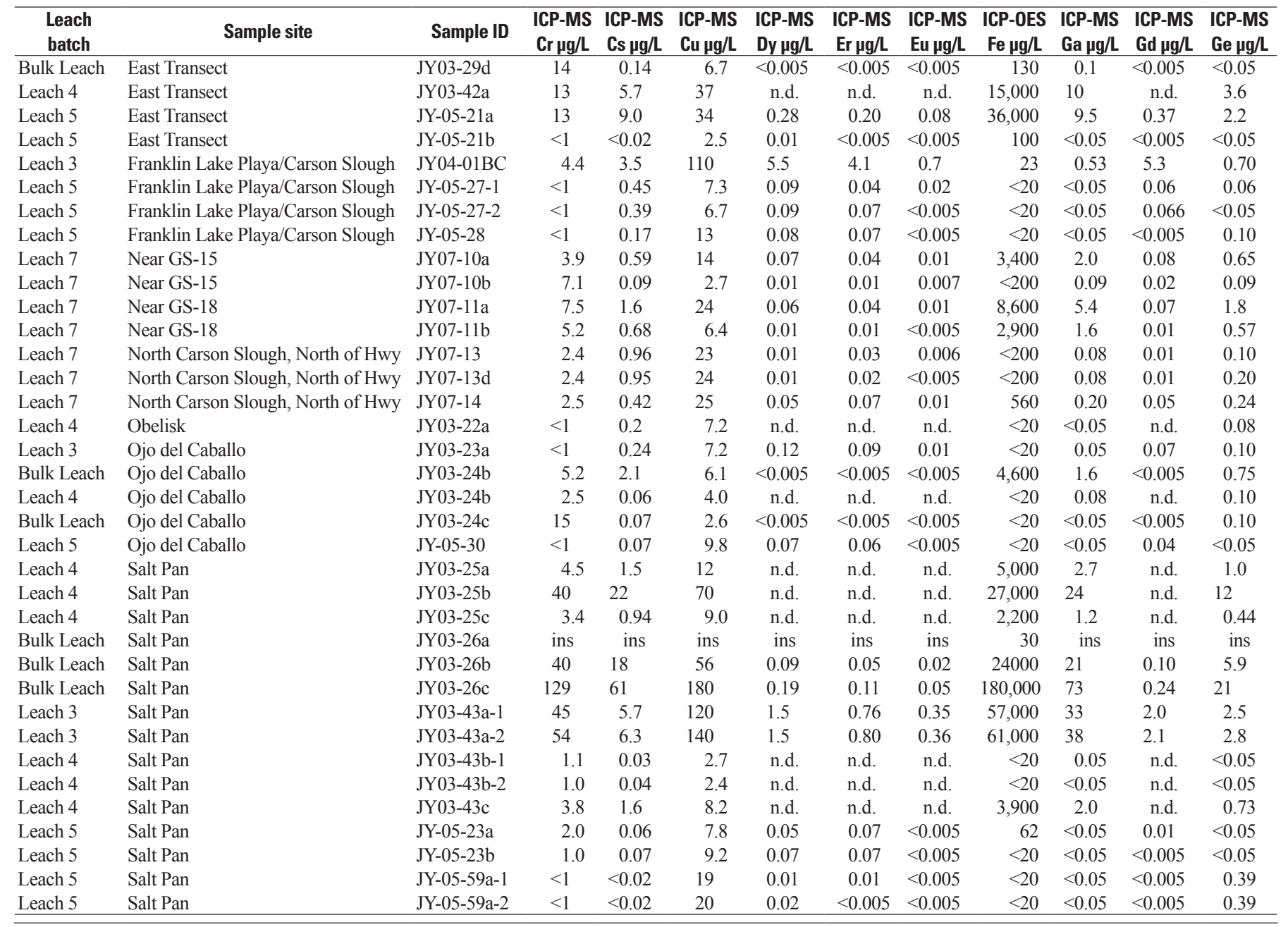


Table 12D. Composition of the DI-leach bulk grab samples. Method of determination (ICP-MS, inductively coupled plasmamass spectrometry; ICP-OES, inductively coupled plasma-optical emission spectrometry; IC, ion chromatograph) is listed above each element.-Continued

[Leach batch, denotes the batch in which the sample was processed and analyzed; Sample site, name of sampling site; Sample ID, unique sample identifier; $-1,-2$, denotes duplicates; $\mathrm{BC}$, bag contents-hydrated salts converted to liquid upon return from field; d, duplicate; n.d., not determined; ins, insufficient sample; $\mu \mathrm{g} / \mathrm{L}$, micrograms per liter; $\mathrm{mg} / \mathrm{L}$, milligrams per liter; $\mathrm{mg} / \mathrm{L} \mathrm{CaCO}_{3}$, milligrams per liter calcium carbonate]

\begin{tabular}{|c|c|c|c|c|c|c|c|c|c|c|c|c|}
\hline $\begin{array}{l}\text { Leach } \\
\text { batch }\end{array}$ & Sample site & Sample ID & $\begin{array}{l}\text { ICP-MS } \\
\text { Ho } \mu \mathrm{g} / \mathrm{L}\end{array}$ & $\begin{array}{c}\text { ICP-0ES } \\
\mathrm{K} \mathrm{mg/L}\end{array}$ & $\begin{array}{l}\text { ICP-MS } \\
\text { La } \mu g / L\end{array}$ & $\begin{array}{l}\text { ICP-MS } \\
\text { Lu } \mu g / L\end{array}$ & $\begin{array}{c}\text { ICP-OES } \\
\text { Li } \mu g / L\end{array}$ & $\begin{array}{l}\text { ICP-MS } \\
\text { Mg mg/L }\end{array}$ & $\begin{array}{l}\text { ICP-MS } \\
\mathrm{Mn} \mu \mathrm{g} / \mathrm{L}\end{array}$ & $\begin{array}{l}\text { ICP-MS } \\
\text { Mo } \mu \mathrm{g} / \mathrm{L}\end{array}$ & $\begin{array}{l}\text { ICP-OES } \\
\text { Na mg/L }\end{array}$ & $\begin{array}{l}\text { ICP-MS } \\
\mathrm{Nb} \mu \mathrm{g} / \mathrm{L}\end{array}$ \\
\hline Bulk Leach & Ash Meadows & JY03-09-2 & $<0.005$ & 120 & $<0.01$ & $<0.1$ & $<10$ & 1.2 & 2.1 & 330 & 4,100 & 1.2 \\
\hline Leach 3 & Ash Meadows & JY03-49a-1 & 0.10 & 39 & 4.7 & $<0.1$ & 1,100 & 340 & 1,800 & 0.43 & 180 & $<0.02$ \\
\hline Leach 4 & Ash Meadows & JY03-49b-1 & n.d. & 44 & $<0.01$ & n.d. & $<10$ & 0.02 & $<0.2$ & 70 & 860 & $<0.2$ \\
\hline Leach 4 & Ash Meadows & JY03-49b-2 & n.d. & 44 & $<0.01$ & n.d. & $<10$ & 0.03 & $<0.2$ & 69 & 870 & $<0.2$ \\
\hline Leach 4 & Ash Meadows & JY03-49c & n.d. & 55 & $<0.01$ & n.d. & $<10$ & 0.02 & $<0.2$ & 150 & 750 & $<0.2$ \\
\hline Leach 4 & Ash Meadows & JY03-50b-1 & n.d. & 25 & $<0.01$ & n.d. & $<10$ & 0.06 & 0.2 & 74 & 1,500 & 0.2 \\
\hline Leach 4 & Ash Meadows & JY03-50b-2 & n.d. & 25 & $<0.01$ & n.d. & $<10$ & 0.05 & $<0.2$ & 74 & 1,500 & 0.2 \\
\hline Leach 4 & Ash Meadows & JY03-50c & n.d. & 39 & 0.03 & n.d. & $<10$ & 0.07 & 0.3 & 140 & 880 & $<0.2$ \\
\hline Leach 5 & Ash Meadows & JY-05-29 & 0.01 & 220 & 0.02 & $<0.1$ & 23 & 2.1 & 9.4 & 5,800 & 6,700 & $<0.2$ \\
\hline Leach 7 & Between GS-18 and East Transect & JY07-12a & 0.10 & 5.2 & 4.1 & $<0.1$ & 140 & 27 & 190 & $<2$ & 82 & $<0.2$ \\
\hline Leach 7 & Between GS-18 and East Transect & JY07-12b & $<0.005$ & 13 & 0.14 & $<0.1$ & 70 & 14 & 110 & $<2$ & 310 & 0.3 \\
\hline Bulk Leach & Discovery & JY03-01-1 & $<0.005$ & 22 & $<0.01$ & $<0.1$ & $<10$ & 0.46 & 4.6 & 190 & 1,900 & 0.4 \\
\hline Bulk Leach & Discovery & JY03-01-2 & $<0.005$ & 23 & $<0.01$ & $<0.1$ & $<10$ & 0.08 & 0.7 & 190 & 1,900 & 0.3 \\
\hline Bulk Leach & Discovery & JY03-01a & $<0.005$ & 8.4 & 0.08 & $<0.1$ & 31 & 5.9 & 84 & 6.8 & 610 & 0.4 \\
\hline Leach 4 & Discovery & JY03-01a & n.d. & 8.3 & 0.15 & n.d. & 35 & 6.5 & 99 & 4.1 & 520 & 0.3 \\
\hline Leach 5 & Discovery & JY-05-15 & 0.04 & 23 & 2.1 & $<0.1$ & 220 & 28 & 470 & 40 & 990 & 0.5 \\
\hline Leach 5 & Discovery & JY-05-16 & 0.08 & 32 & 5.9 & $<0.1$ & 450 & 64 & 970 & 9.1 & 300 & 2.3 \\
\hline Leach 7 & Discovery & JY07-09 & 0.006 & 27 & 0.16 & $<0.1$ & $<50$ & 0.78 & 15 & 120 & 4,100 & 1.6 \\
\hline Leach 5 & Dust Trap Array & $\mathrm{T}-69$ & 7.8 & 45 & 390 & 2.5 & 240 & 110 & 5,800 & 4.5 & 180 & 0.9 \\
\hline Leach 4 & East of Coppice & JY03-32a & n.d. & 24 & 0.02 & n.d. & $<10$ & 0.02 & $<0.2$ & 340 & 1,800 & 0.4 \\
\hline Leach 4 & East of East Transect & JY03-28a-1 & n.d. & 23 & 0.18 & n.d. & 380 & 82 & 770 & 8.9 & 250 & 0.8 \\
\hline Leach 4 & East of East Transect & JY03-28a-2 & n.d. & 23 & 0.17 & n.d. & 380 & 83 & 790 & 8.4 & 250 & 0.9 \\
\hline Leach 4 & East of East Transect & JY03-28b & n.d. & 26 & 0.61 & n.d. & 690 & 160 & 1,500 & 4.6 & 160 & 9.2 \\
\hline Leach 4 & East of East Transect & JY03-28c & n.d. & 21 & 0.33 & n.d. & 220 & 110 & 1,100 & 7.4 & 240 & 3.8 \\
\hline
\end{tabular}


Table 12D. Composition of the DI-leach bulk grab samples. Method of determination (ICP-MS, inductively coupled plasmamass spectrometry; ICP-OES, inductively coupled plasma-optical emission spectrometry; IC, ion chromatograph) is listed above each element.-Continued

[Leach batch, denotes the batch in which the sample was processed and analyzed; Sample site, name of sampling site; Sample ID, unique sample identifier; $-1,-2$, denotes duplicates; $\mathrm{BC}$, bag contents-hydrated salts converted to liquid upon return from field; d, duplicate; n.d., not determined; ins, insufficient sample; $\mu \mathrm{g} / \mathrm{L}$, micrograms per liter; $\mathrm{mg} / \mathrm{L}$, milligrams per liter; $\mathrm{mg} / \mathrm{L} \mathrm{CaCO}$, milligrams per liter calcium carbonate]

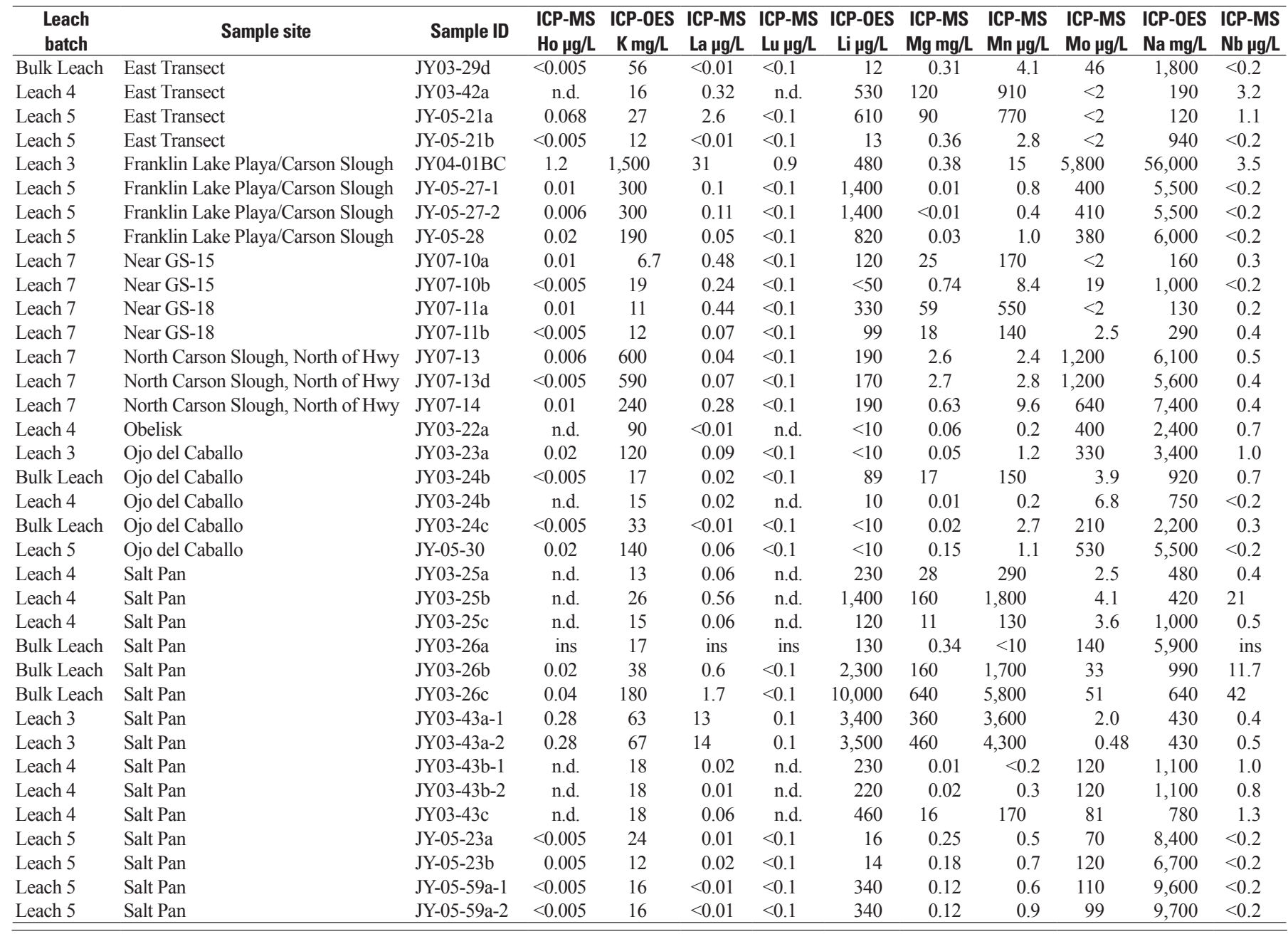


Table 12D. Composition of the DI-leach bulk grab samples. Method of determination (ICP-MS, inductively coupled plasmamass spectrometry; ICP-OES, inductively coupled plasma-optical emission spectrometry; IC, ion chromatograph) is listed above each element.-Continued

[Leach batch, denotes the batch in which the sample was processed and analyzed; Sample site, name of sampling site; Sample ID, unique sample identifier; -1, -2, denotes duplicates; BC, bag contents-hydrated salts converted to liquid upon return from field; d, duplicate; n.d., not determined; ins, insufficient sample; $\mu \mathrm{g} / \mathrm{L}$, micrograms per liter; $\mathrm{mg} / \mathrm{L}$, milligrams per liter; $\mathrm{mg} / \mathrm{L} \mathrm{CaCO}_{3}$, milligrams per liter calcium carbonate]

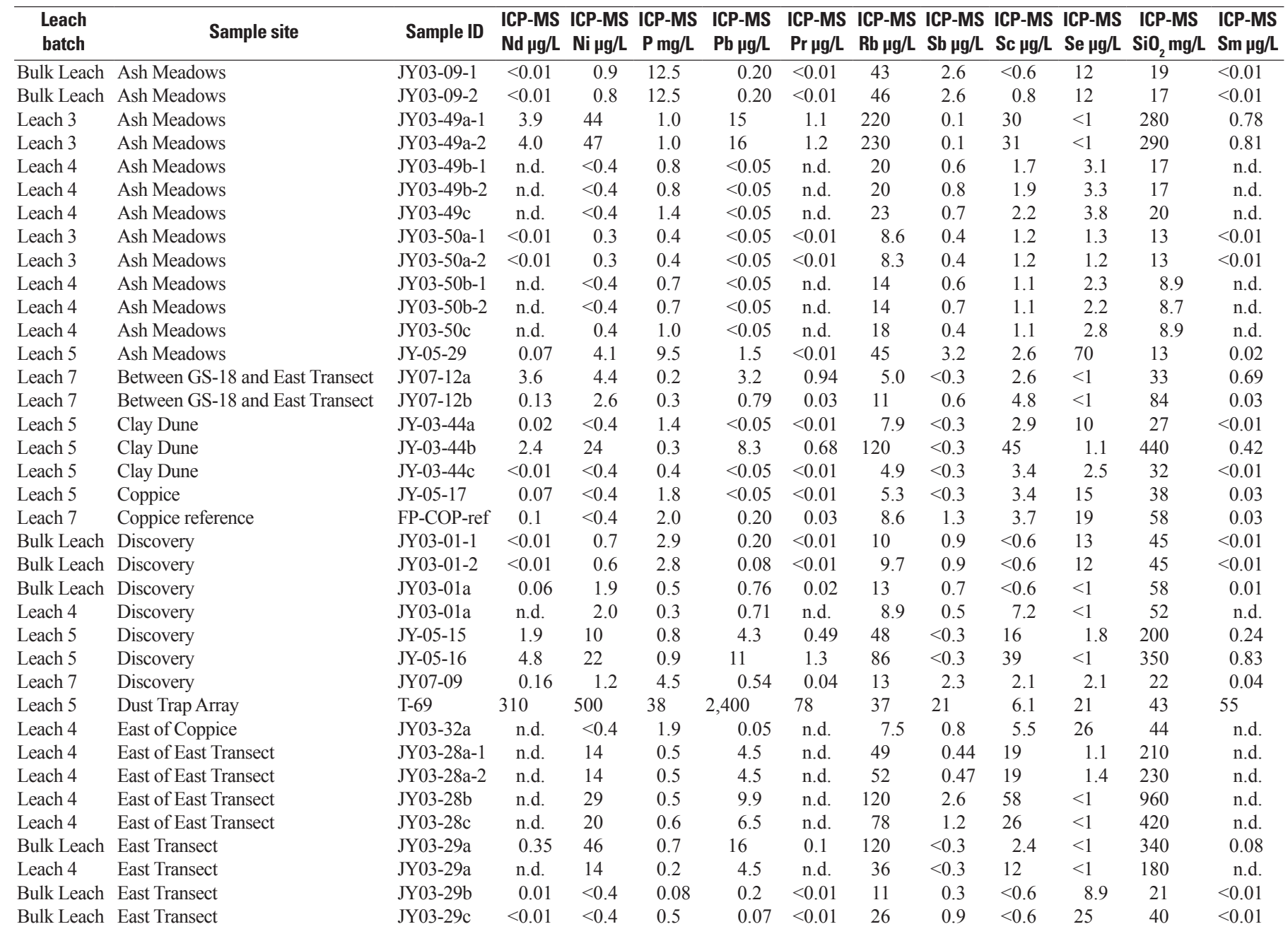


Table 12D. Composition of the DI-leach bulk grab samples. Method of determination (ICP-MS, inductively coupled plasmamass spectrometry; ICP-0ES, inductively coupled plasma-optical emission spectrometry; IC, ion chromatograph) is listed above each element.-Continued

[Leach batch, denotes the batch in which the sample was processed and analyzed; Sample site, name of sampling site; Sample ID, unique sample identifier; $-1,-2$, denotes duplicates; BC, bag contents-hydrated salts converted to liquid upon return from field; $d$, duplicate; n.d., not determined; ins, insufficient sample; $\mu \mathrm{g} / \mathrm{L}$, micrograms per liter; $\mathrm{mg} / \mathrm{L}$, milligrams per liter; $\mathrm{mg} / \mathrm{L} \mathrm{CaCO}{ }_{3}$, milligrams per liter calcium carbonate]

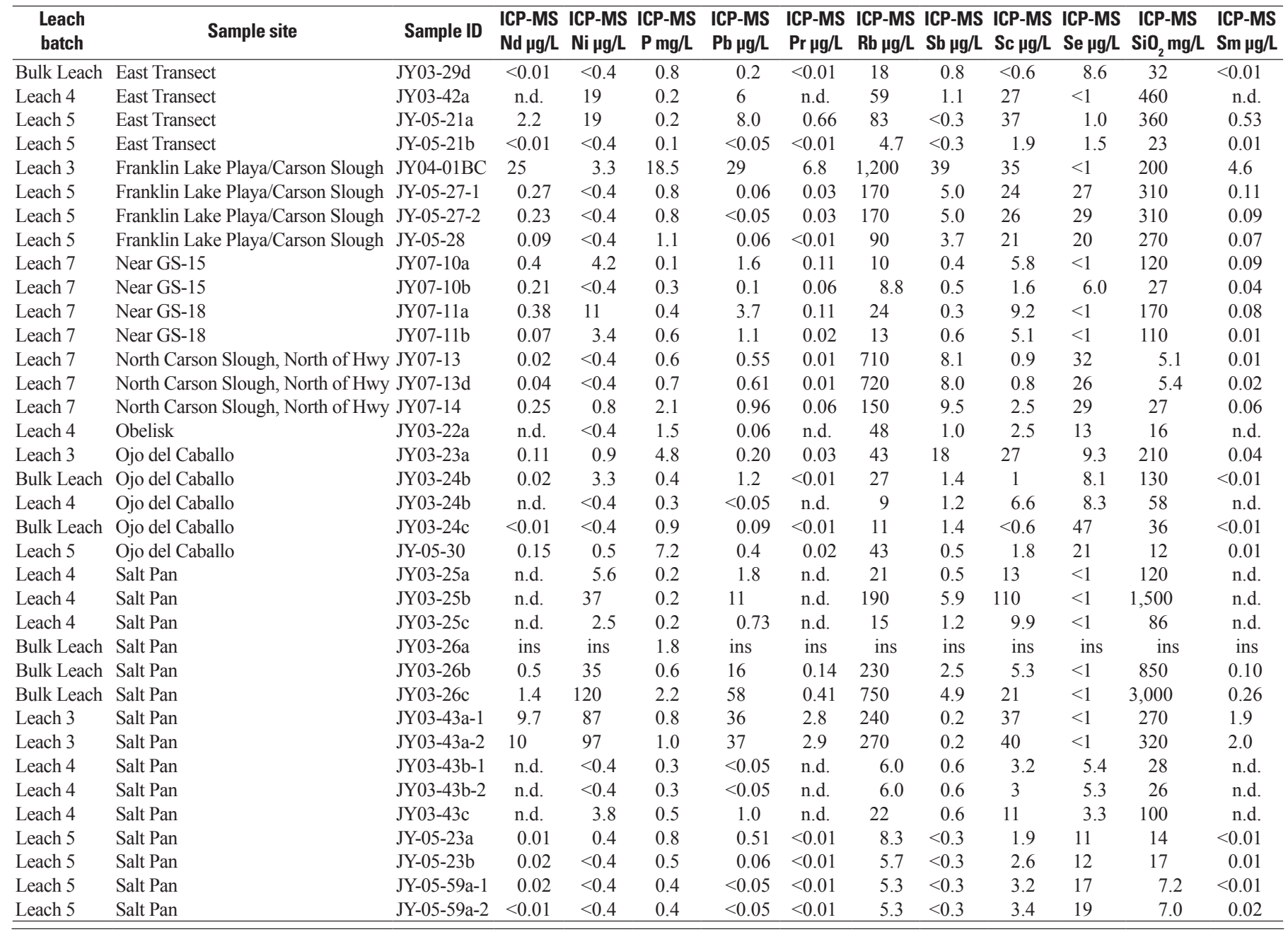


Table 12D. Composition of the DI-leach bulk grab samples. Method of determination (ICP-MS, inductively coupled plasmamass spectrometry; ICP-OES, inductively coupled plasma-optical emission spectrometry; IC, ion chromatograph) is listed above each element.-Continued

[Leach batch, denotes the batch in which the sample was processed and analyzed; Sample site, name of sampling site; Sample ID, unique sample identifier; $-1,-2$, denotes duplicates; BC, bag contents-hydrated salts converted to liquid upon return from field; d, duplicate; n.d., not determined; ins, insufficient sample; $\mu \mathrm{g} / \mathrm{L}$, micrograms per liter; $\mathrm{mg} / \mathrm{L}$, milligrams per liter; $\mathrm{mg} / \mathrm{L} \mathrm{CaCO}_{3}$, milligrams per liter calcium carbonate]

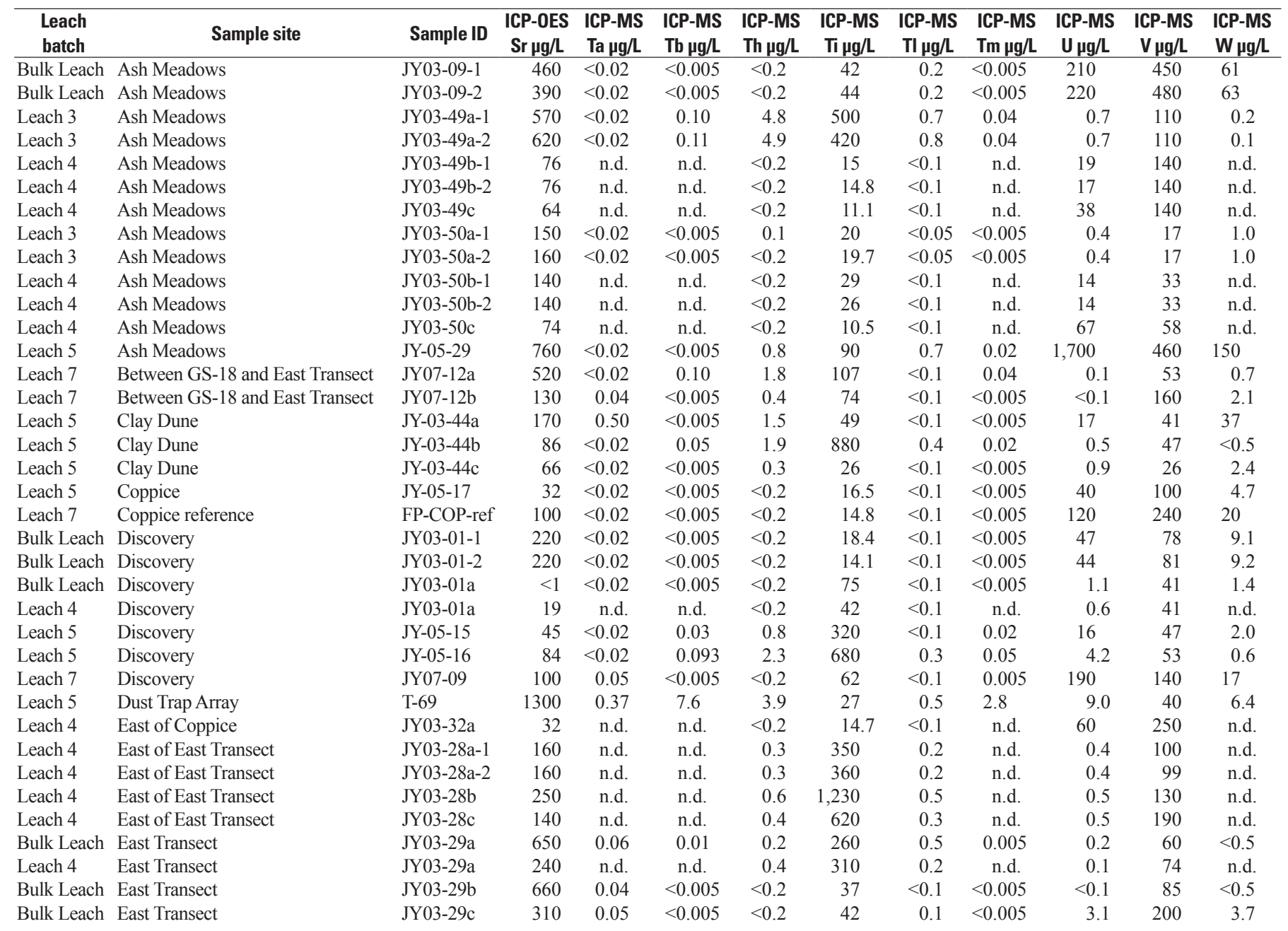


Table 12D. Composition of the DI-leach bulk grab samples. Method of determination (ICP-MS, inductively coupled plasmamass spectrometry; ICP-0ES, inductively coupled plasma-optical emission spectrometry; IC, ion chromatograph) is listed above each element.-Continued

[Leach batch, denotes the batch in which the sample was processed and analyzed; Sample site, name of sampling site; Sample ID, unique sample identifier; $-1,-2$, denotes duplicates; $\mathrm{BC}$, bag contents-hydrated salts converted to liquid upon return from field; d, duplicate; n.d., not determined; ins, insufficient sample; $\mu \mathrm{g} / \mathrm{L}$, micrograms per liter; $\mathrm{mg} / \mathrm{L}$, milligrams per liter; $\mathrm{mg} / \mathrm{L} \mathrm{CaCO}_{3}$, milligrams per liter calcium carbonate]

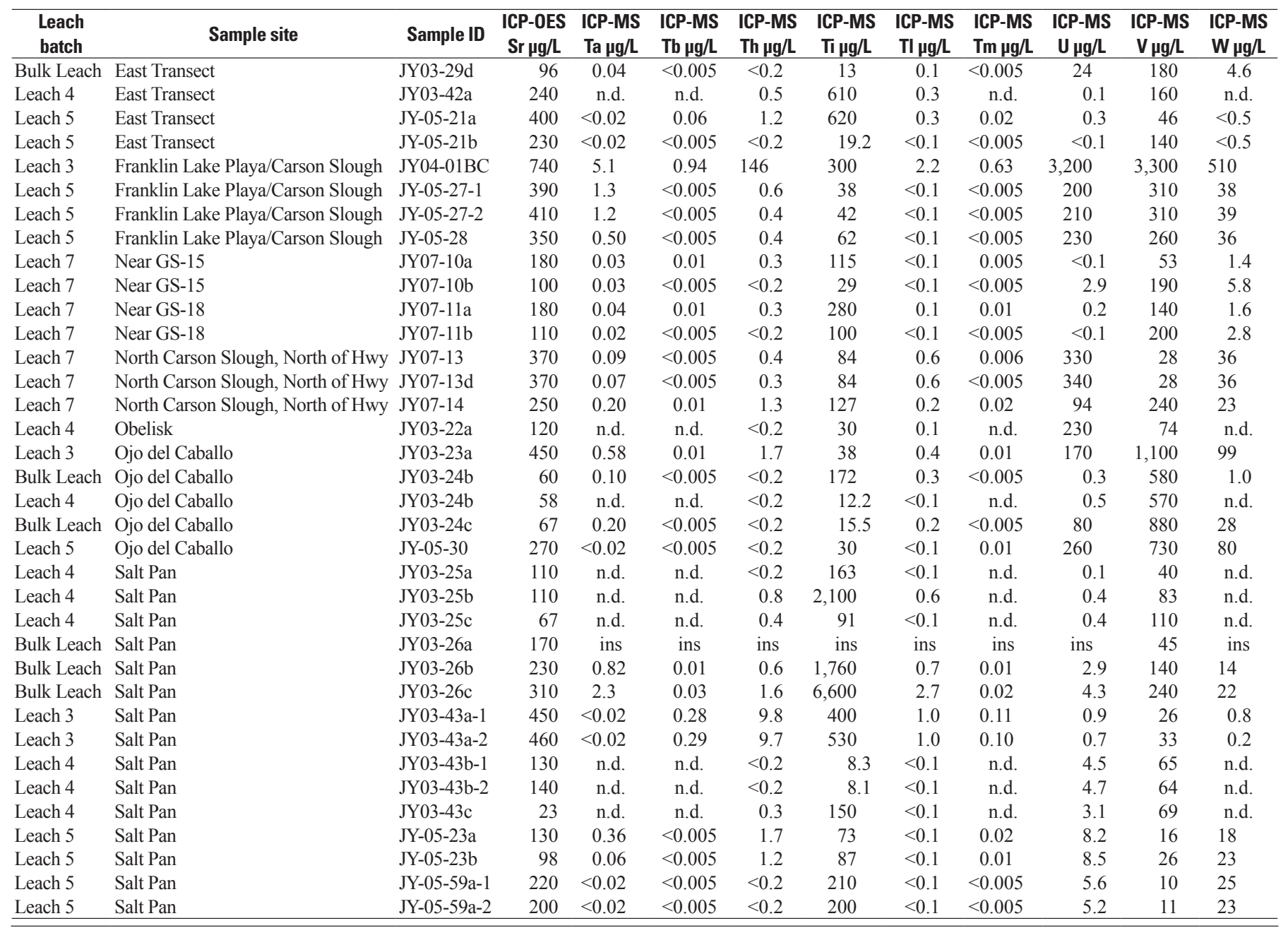


Table 12D. Composition of the DI-leach bulk grab samples. Method of determination (ICP-MS, inductively coupled plasmamass spectrometry; ICP-OES, inductively coupled plasma-optical emission spectrometry; IC, ion chromatograph) is listed above each element.-Continued

[Leach batch, denotes the batch in which the sample was processed and analyzed; Sample site, name of sampling site; Sample ID, unique sample identifier; -1, -2, denotes duplicates; BC, bag contents-hydrated salts converted to liquid upon return from field; d, duplicate; n.d., not determined; ins, insufficient sample; $\mu \mathrm{g} / \mathrm{L}$, micrograms per liter; $\mathrm{mg} / \mathrm{L}$, milligrams per liter; $\mathrm{mg} / \mathrm{L} \mathrm{CaCO}_{3}$, milligrams per liter calcium carbonate]

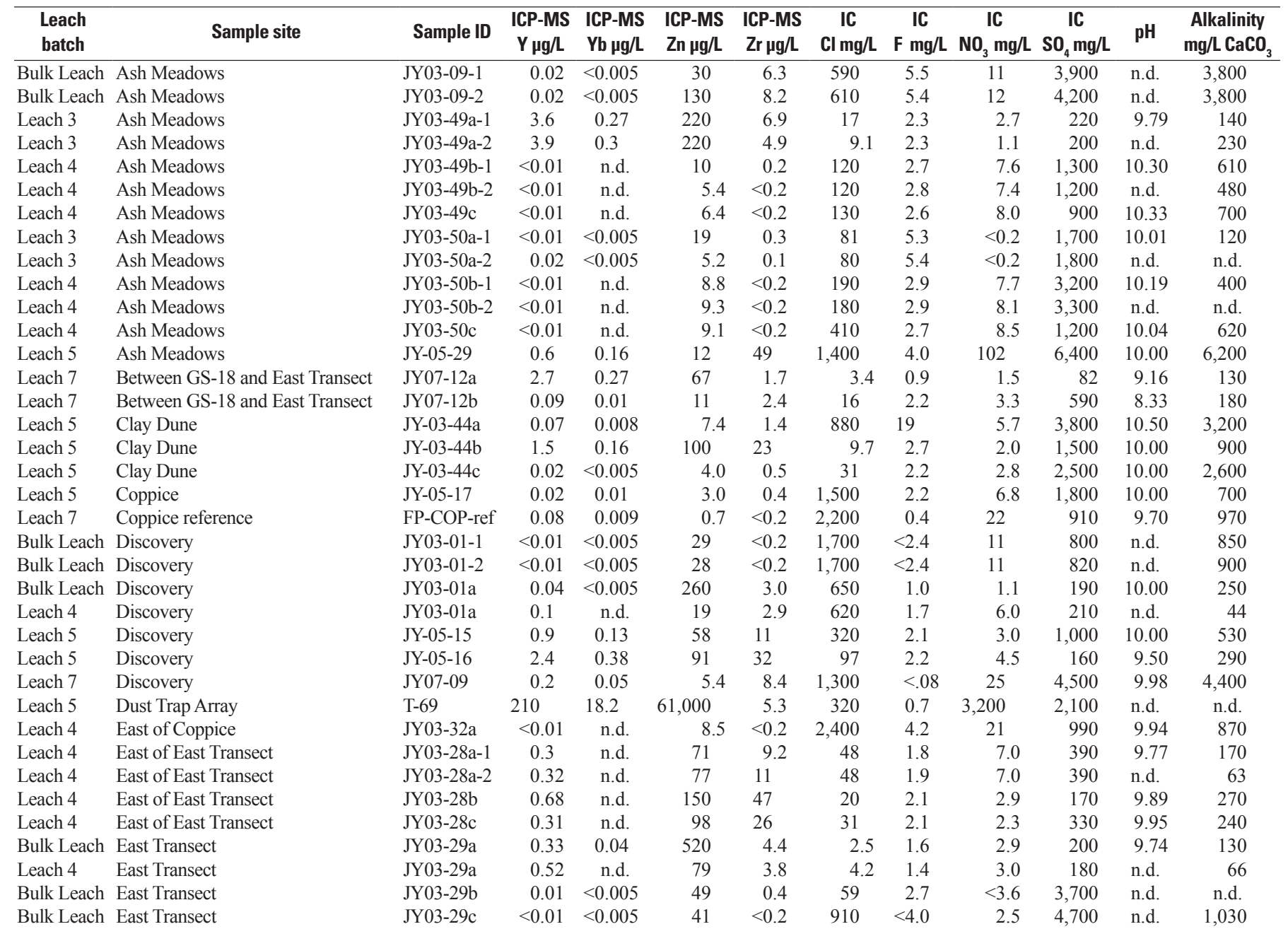


Table 12D. Composition of the DI-leach bulk grab samples. Method of determination (ICP-MS, inductively coupled plasmamass spectrometry; ICP-OES, inductively coupled plasma-optical emission spectrometry; IC, ion chromatograph) is listed above each element.-Continued

[Leach batch, denotes the batch in which the sample was processed and analyzed; Sample site, name of sampling site; Sample ID, unique sample identifier; -1, -2 , denotes duplicates; $\mathrm{BC}$, bag contents-hydrated salts converted to liquid upon return from field; d, duplicate; n.d., not determined; ins, insufficient sample; $\mu \mathrm{g} / \mathrm{L}$, micrograms per liter; $\mathrm{mg} / \mathrm{L}$, milligrams per liter; $\mathrm{mg} / \mathrm{L} \mathrm{CaCO}{ }_{3}$, milligrams per liter calcium carbonate]

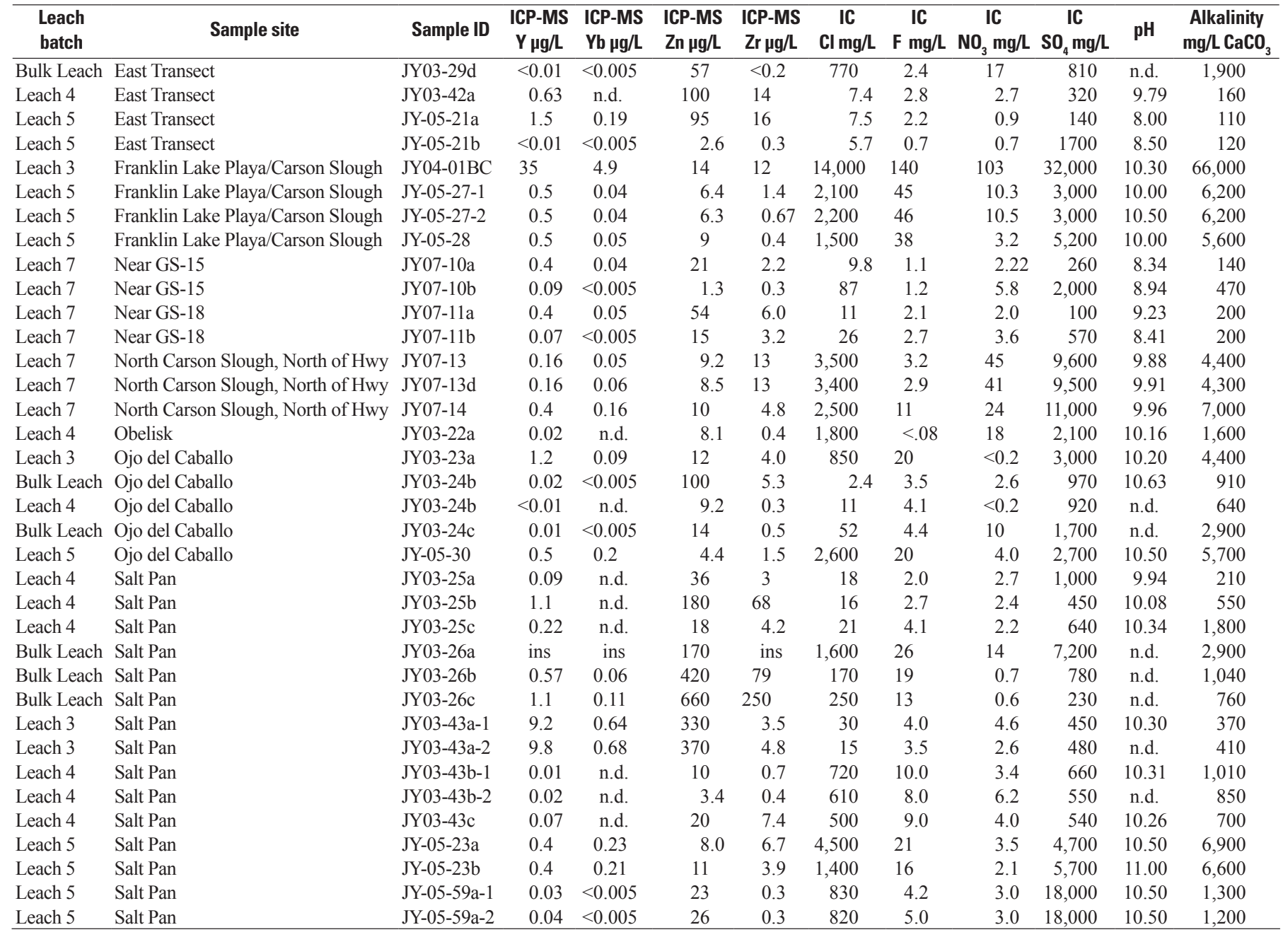


Table 12E. Composition of the nitric acid leach bulk grab samples. Method of determination (ICP-MS, inductively coupled plasmamass spectrometry; ICP-OES, inductively coupled plasma-optical emission spectrometry) is listed above each element.

[Leach batch, denotes the batch in which the sample was processed and analyzed; Sample site, name of sampling site; Sample ID, unique sample identifier; $-1,-2$, denotes duplicates; BC, bag contents-hydrated salts converted to liquid upon return from field; d, duplicate; n.r., not reported; ins, insufficient sample; $\mu \mathrm{g} / \mathrm{L}$, micrograms per liter; $\mathrm{mg} / \mathrm{L}$, milligrams per liter; $\mathrm{mg} / \mathrm{L} \mathrm{CaCO}_{3}$, milligrams per liter calcium carbonate]

\begin{tabular}{|c|c|c|c|c|c|c|c|c|c|c|c|c|}
\hline $\begin{array}{l}\text { Leach } \\
\text { batch }\end{array}$ & Sample site & Sample ID & $\begin{array}{l}\text { ICP-MS } \\
\mathrm{Ag} \mu \mathrm{g} / \mathrm{L}\end{array}$ & $\begin{array}{c}\text { ICP-OES } \\
\text { Al } \mu g / L\end{array}$ & $\begin{array}{l}\text { ICP-MS } \\
\text { As } \mu \mathrm{g} / \mathrm{L}\end{array}$ & $\begin{array}{c}\text { ICP-OES } \\
\text { B } \mu \mathrm{g} / \mathrm{L}\end{array}$ & $\begin{array}{l}\text { ICP-MS } \\
\text { Ba } \mu g / L\end{array}$ & $\begin{array}{l}\text { ICP-MS } \\
\text { Be } \mu \mathrm{g} / \mathrm{L}\end{array}$ & $\begin{array}{c}\text { ICP-MS } \\
\mathrm{Bi} \mu \mathrm{g} / \mathrm{L}\end{array}$ & $\begin{array}{l}\text { ICP-MS } \\
\text { Ca mg/L }\end{array}$ & $\begin{array}{l}\text { ICP-MS } \\
\mathrm{Cd} \mu \mathrm{g} / \mathrm{L}\end{array}$ & $\begin{array}{l}\text { ICP-MS } \\
\text { Ce } \mu \mathrm{g} / \mathrm{L}\end{array}$ \\
\hline Bulk Leach & Ash Meadows & JY03-09-2 & $<3$ & 2,500 & 590 & 13,000 & 2,700 & 0.8 & $<0.2$ & 3,300 & 0.97 & n.r. \\
\hline Leach 3 & Ash Meadows & JY03-49a-1 & $<3$ & 1,200 & 57 & 920 & 2,000 & 0.3 & 0.01 & 3,900 & 0.92 & 94 \\
\hline Leach 4 & Ash Meadows & JY03-49b-1 & $<3$ & 3,300 & 100 & 2,700 & 2,100 & 0.7 & $<0.2$ & 2,800 & 0.47 & 170 \\
\hline Leach 4 & Ash Meadows & JY03-49b-2 & $<3$ & 2,800 & 98 & 2,800 & 2,000 & 0.8 & $<0.2$ & 2,800 & 0.63 & 190 \\
\hline Leach 4 & Ash Meadows & JY03-49c & $<3$ & 23,000 & 94 & 2,800 & 2,600 & 1.8 & $<0.2$ & 2,700 & 1.6 & 340 \\
\hline Leach 4 & Ash Meadows & JY03-50b-1 & $<3$ & 2,900 & 68 & 1,200 & 2,600 & 0.5 & $<0.2$ & 2,800 & 0.9 & 130 \\
\hline Leach 4 & Ash Meadows & JY03-50b-2 & $<3$ & 1,800 & 72 & 1,100 & 2,600 & 0.6 & $<0.2$ & 2,600 & 0.75 & 94 \\
\hline Leach 4 & Ash Meadows & JY03-50c & $<3$ & 30,000 & 120 & 1,500 & 2,900 & 1.6 & $<0.2$ & 2,200 & 1.9 & 310 \\
\hline Leach 5 & Ash Meadows & JY-05-29 & $<3$ & 270 & 270 & 16,000 & 1,200 & 0.2 & $<0.2$ & 3,400 & 0.56 & 11 \\
\hline Leach 7 & Between GS-18 and East Transect & JY07-12a & $<1$ & 31,000 & 31 & 430 & 1,500 & 1.8 & $<0.2$ & 1,600 & 2.3 & 380 \\
\hline Leach 7 & Between GS-18 and East Transect & JY07-12b & $<1$ & 87,000 & 39 & 1,100 & 1,500 & 5.0 & $<0.2$ & 1,400 & 2.5 & 430 \\
\hline Leach 7 & Coppice reference & FP-COP-refA & $<1$ & 120,000 & 170 & 7,200 & 2,100 & 0 & $<0.2$ & 1,300 & 4.8 & 610 \\
\hline Bulk Leach & Discovery & JY03-01-1 & $<3$ & 78,000 & 90 & 4,500 & 2,600 & 8 & $<0.2$ & 2,100 & 5.7 & n.r. \\
\hline Bulk Leach & Discovery & JY03-01-2 & $<3$ & 80,000 & 85 & 4,500 & 3,200 & 6.4 & $<0.2$ & 2,200 & 64 & n.r. \\
\hline Bulk Leach & Discovery & JY03-01a & $<3$ & 78,000 & 43 & 2,000 & 4,600 & 5.7 & $<0.2$ & 2,600 & 6.0 & n.r. \\
\hline Leach 4 & Discovery & JY03-01a & $<3$ & 76,000 & 48 & 2,600 & 3,200 & 5.1 & $<0.2$ & 2,600 & 4.6 & 1,100 \\
\hline Leach 5 & Discovery & JY-05-15 & $<3$ & 85,000 & 86 & 5,300 & 2,400 & 5.1 & $<0.2$ & 2,000 & 5.5 & 770 \\
\hline Leach 5 & Discovery & JY-05-16 & $<3$ & 70,000 & 62 & 3,400 & 2,800 & 4.6 & $<0.2$ & 2,300 & 6.5 & 860 \\
\hline Leach 7 & Discovery & JY07-09 & $<1$ & 39,000 & 52 & 1,700 & 2,800 & 3.4 & $<0.2$ & 2,200 & 4.3 & 710 \\
\hline Leach 5 & Dust Trap Array & $\mathrm{T}-69$ & $<3$ & $<50$ & 43 & 5,800 & 73 & 0.05 & $<0.2$ & 200 & 0.89 & 0.08 \\
\hline Leach 4 & East of Coppice & JY03-32a & $<3$ & 120,000 & 83 & 6,600 & 3,100 & 8.2 & $<0.2$ & 1,800 & 5.4 & 960 \\
\hline Leach 4 & East of East Transect & JY03-28a-1 & $<3$ & 120,000 & 64 & 2,700 & 2,900 & 4.7 & $<0.2$ & 1,900 & 3.3 & 800 \\
\hline Leach 4 & East of East Transect & JY03-28a-2 & $<3$ & 160,000 & 67 & 2,700 & 3,100 & 5.1 & $<0.2$ & 2,000 & 3.4 & 830 \\
\hline Leach 4 & East of East Transect & JY03-28b & $<3$ & 170,000 & 65 & 3,000 & 2,900 & 5.4 & $<0.2$ & 2,000 & 3.4 & 860 \\
\hline
\end{tabular}


Table 12E. Composition of the nitric acid leach bulk grab samples. Method of determination (ICP-MS, inductively coupled plasmamass spectrometry; ICP-OES, inductively coupled plasma-optical emission spectrometry) is listed above each element.-Continued

[Leach batch, denotes the batch in which the sample was processed and analyzed; Sample site, name of sampling site; Sample ID, unique sample identifier; $-1,-2$, denotes duplicates; BC, bag contents-hydrated salts converted to liquid upon return from field; d, duplicate; n.r., not reported; ins, insufficient sample; $\mu \mathrm{g} / \mathrm{L}$, micrograms per liter; $\mathrm{mg} / \mathrm{L}$, milligrams per liter; $\mathrm{mg} / \mathrm{L} \mathrm{CaCO}$, milligrams per liter calcium carbonate]

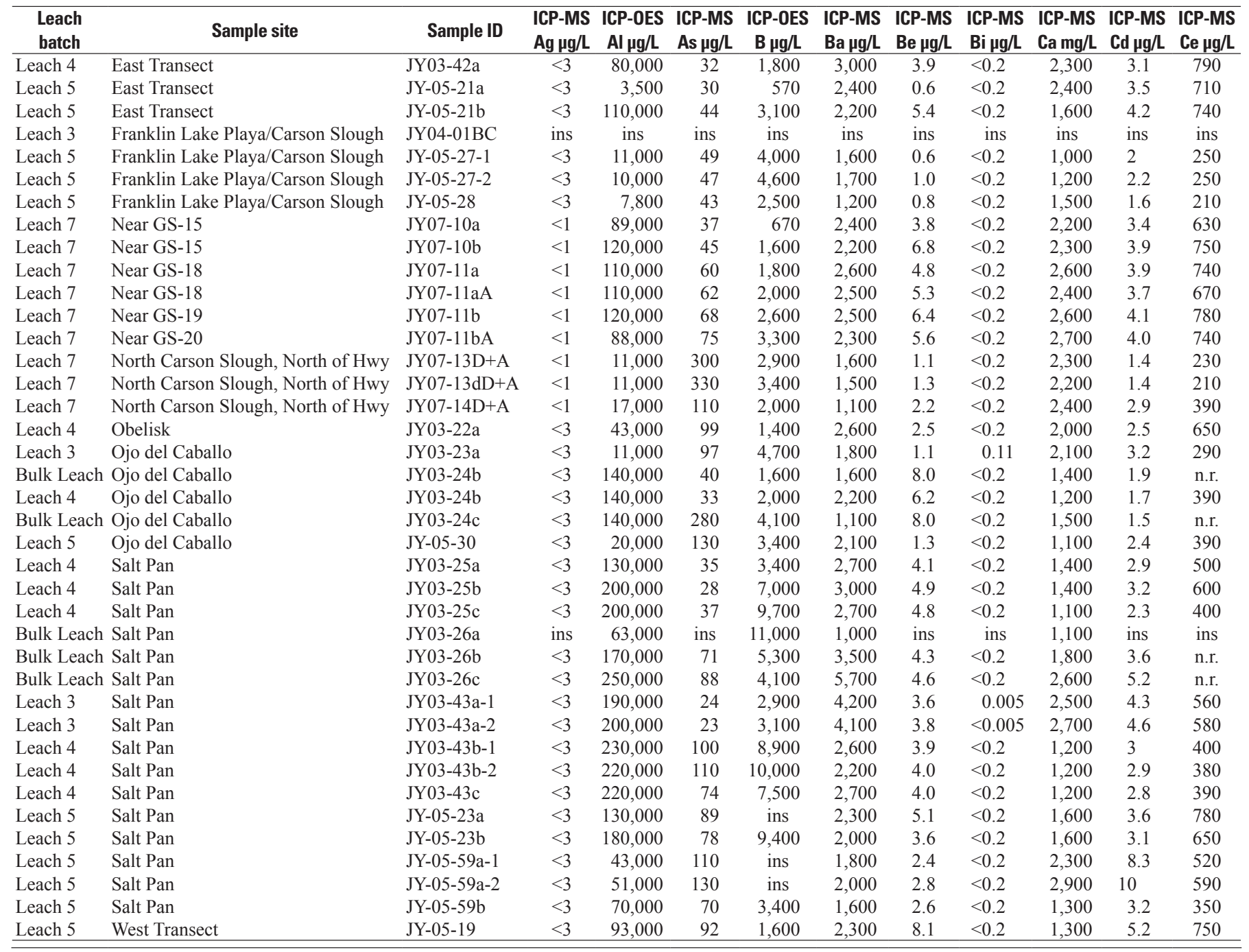


Table 12E. Composition of the nitric acid leach bulk grab samples. Method of determination (ICP-MS, inductively coupled plasmamass spectrometry; ICP-OES, inductively coupled plasma-optical emission spectrometry) is listed above each element.-Continued

[Leach batch, denotes the batch in which the sample was processed and analyzed; Sample site, name of sampling site; Sample ID, unique sample identifier; $-1,-2$, denotes duplicates; BC, bag contents-hydrated salts converted to liquid upon return from field; d, duplicate; n.r., not reported; ins, insufficient sample; $\mu \mathrm{g} / \mathrm{L}$, micrograms per liter; $\mathrm{mg} / \mathrm{L}$, milligrams per liter; $\mathrm{mg} / \mathrm{L} \mathrm{CaCO}_{3}$, milligrams per liter calcium carbonate]

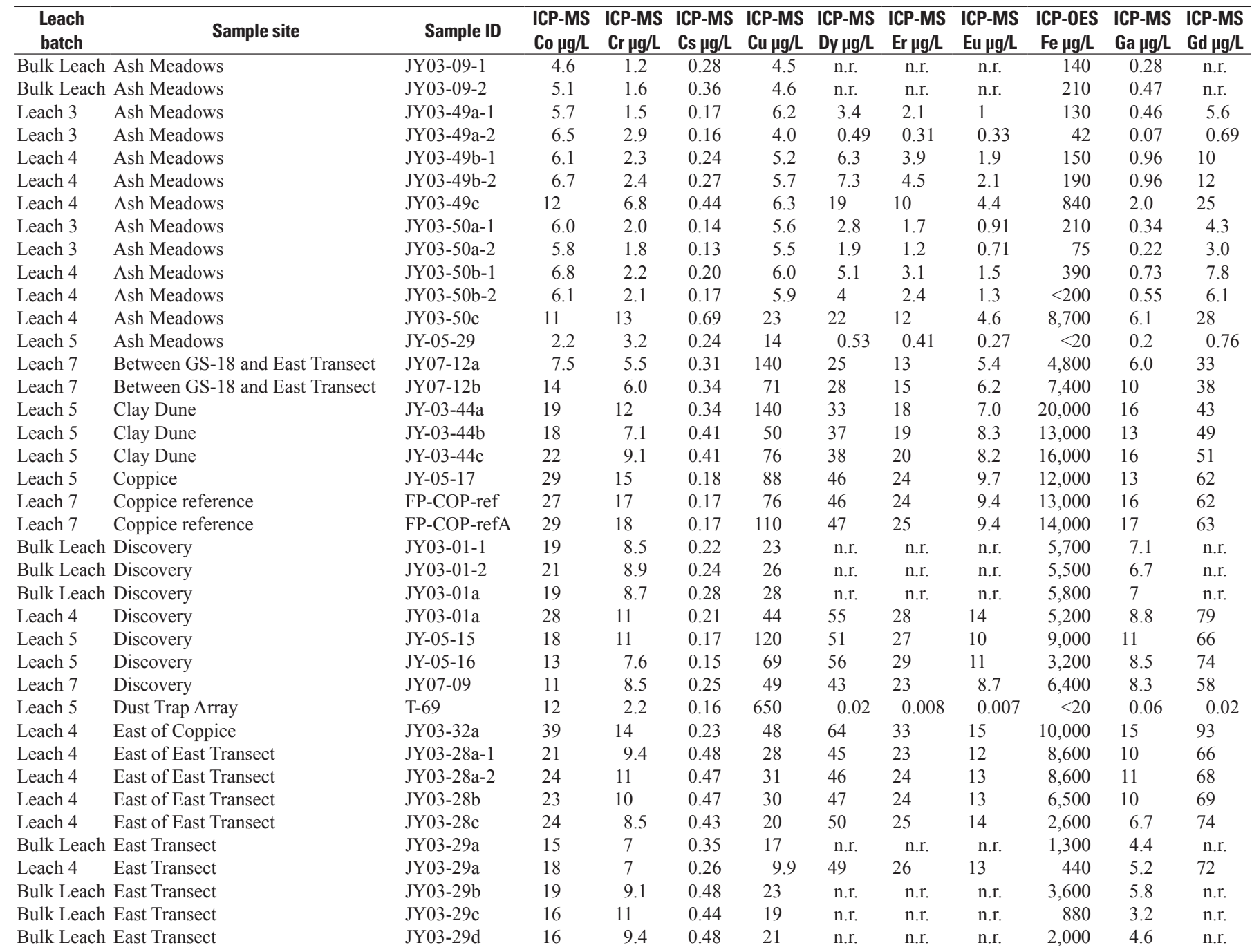


Table 12E. Composition of the nitric acid leach bulk grab samples. Method of determination (ICP-MS, inductively coupled plasmamass spectrometry; ICP-OES, inductively coupled plasma-optical emission spectrometry) is listed above each element.-Continued

[Leach batch, denotes the batch in which the sample was processed and analyzed; Sample site, name of sampling site; Sample ID, unique sample identifier; $-1,-2$, denotes duplicates; BC, bag contents-hydrated salts converted to liquid upon return from field; d, duplicate; n.r., not reported; ins, insufficient sample; $\mu \mathrm{g} / \mathrm{L}$, micrograms per liter; $\mathrm{mg} / \mathrm{L}$, milligrams per liter; $\mathrm{mg} / \mathrm{L} \mathrm{CaCO}$, milligrams per liter calcium carbonate]

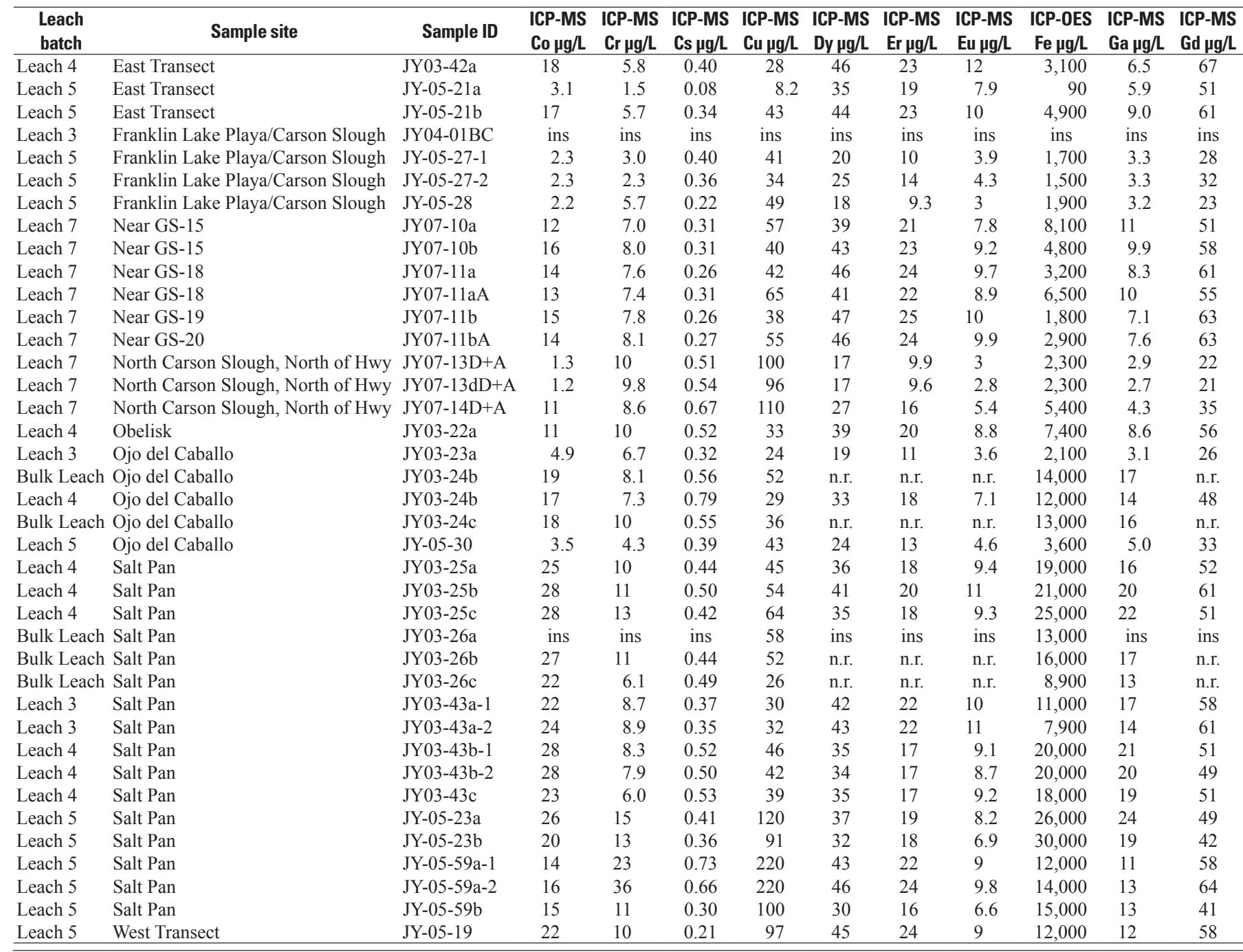


Table 12E. Composition of the nitric acid leach bulk grab samples. Method of determination (ICP-MS, inductively coupled plasmamass spectrometry; ICP-OES, inductively coupled plasma-optical emission spectrometry) is listed above each element.-Continued

[Leach batch, denotes the batch in which the sample was processed and analyzed; Sample site, name of sampling site; Sample ID, unique sample identifier; $-1,-2$, denotes duplicates; BC, bag contents-hydrated salts converted to liquid upon return from field; d, duplicate; n.r., not reported; ins, insufficient sample; $\mu \mathrm{g} / \mathrm{L}$, micrograms per liter; $\mathrm{mg} / \mathrm{L}$, milligrams per liter; $\mathrm{mg} / \mathrm{L} \mathrm{CaCO}_{3}$, milligrams per liter calcium carbonate]

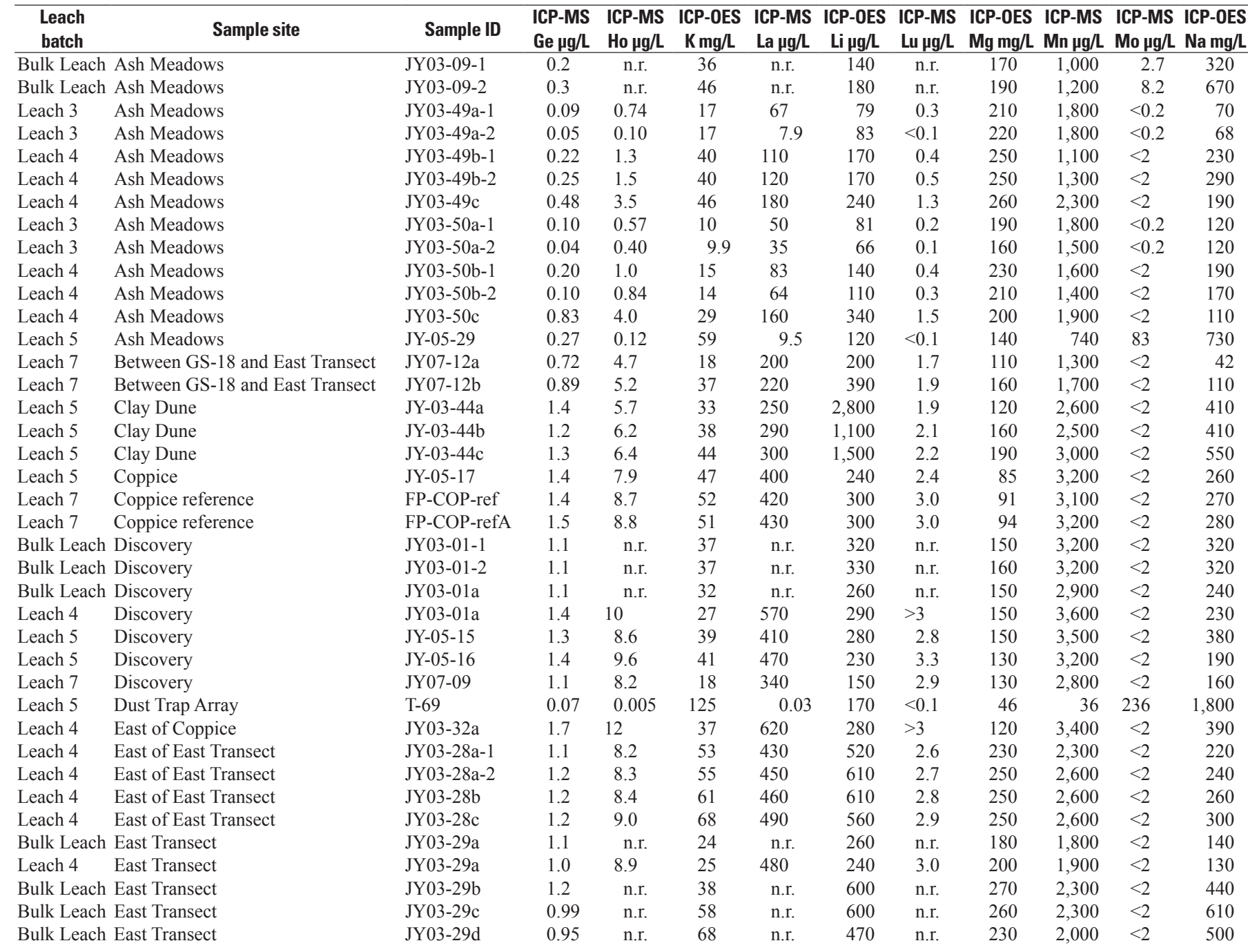


Table 12E. Composition of the nitric acid leach bulk grab samples. Method of determination (ICP-MS, inductively coupled plasmamass spectrometry; ICP-OES, inductively coupled plasma-optical emission spectrometry) is listed above each element.-Continued

[Leach batch, denotes the batch in which the sample was processed and analyzed; Sample site, name of sampling site; Sample ID, unique sample identifier; $-1,-2$, denotes duplicates; BC, bag contents-hydrated salts converted to liquid upon return from field; d, duplicate; n.r., not reported; ins, insufficient sample; $\mu \mathrm{g} / \mathrm{L}$, micrograms per liter; $\mathrm{mg} / \mathrm{L}$, milligrams per liter; $\mathrm{mg} / \mathrm{L} \mathrm{CaCO}_{3}$, milligrams per liter calcium carbonate]

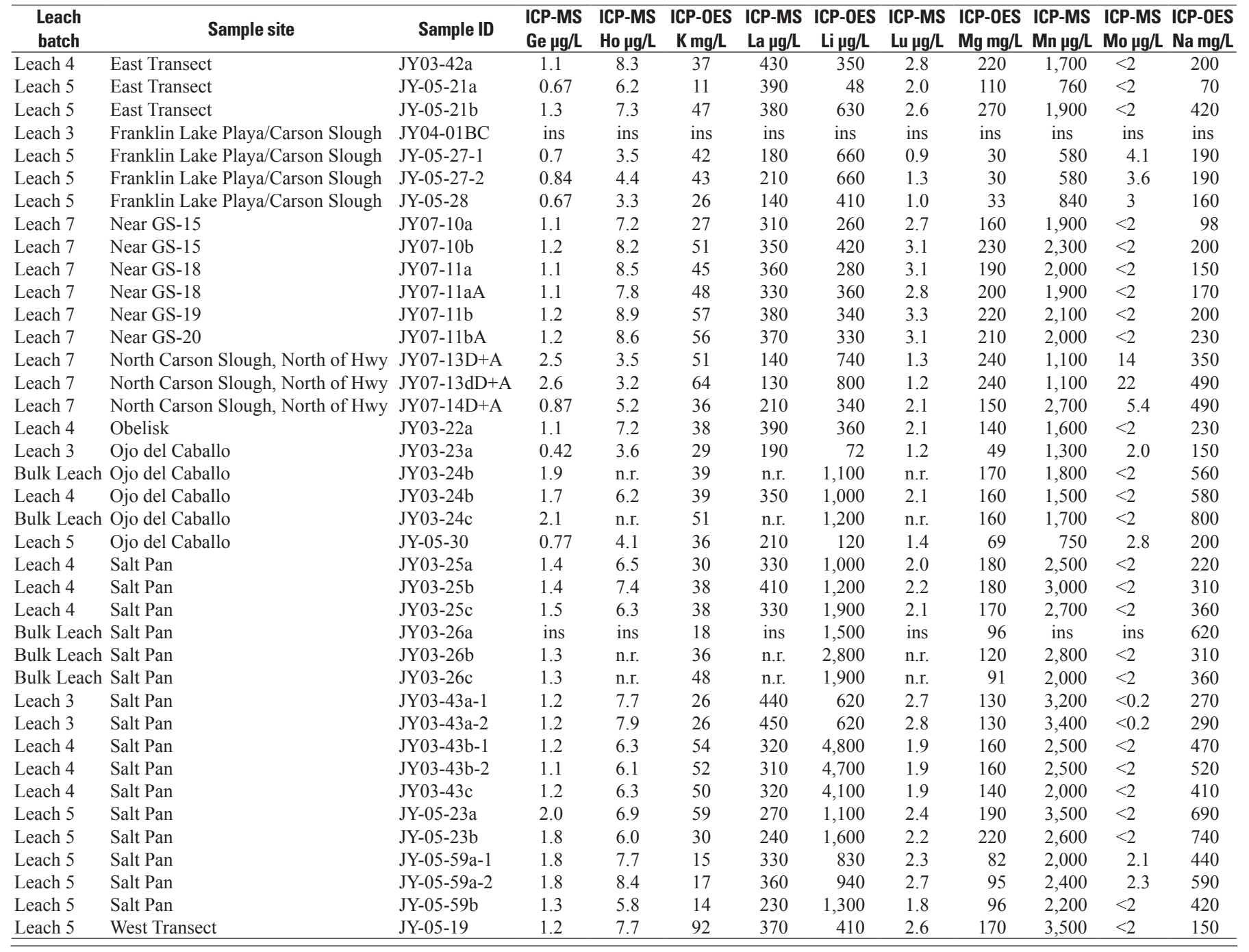


Table 12E. Composition of the nitric acid leach bulk grab samples. Method of determination (ICP-MS, inductively coupled plasmamass spectrometry; ICP-OES, inductively coupled plasma-optical emission spectrometry) is listed above each element.-Continued

[Leach batch, denotes the batch in which the sample was processed and analyzed; Sample site, name of sampling site; Sample ID, unique sample identifier; $-1,-2$, denotes duplicates; BC, bag contents-hydrated salts converted to liquid upon return from field; d, duplicate; n.r., not reported; ins, insufficient sample; $\mu \mathrm{g} / \mathrm{L}$, micrograms per liter; $\mathrm{mg} / \mathrm{L}$, milligrams per liter; $\mathrm{mg} / \mathrm{L} \mathrm{CaCO}_{3}$, milligrams per liter calcium carbonate]

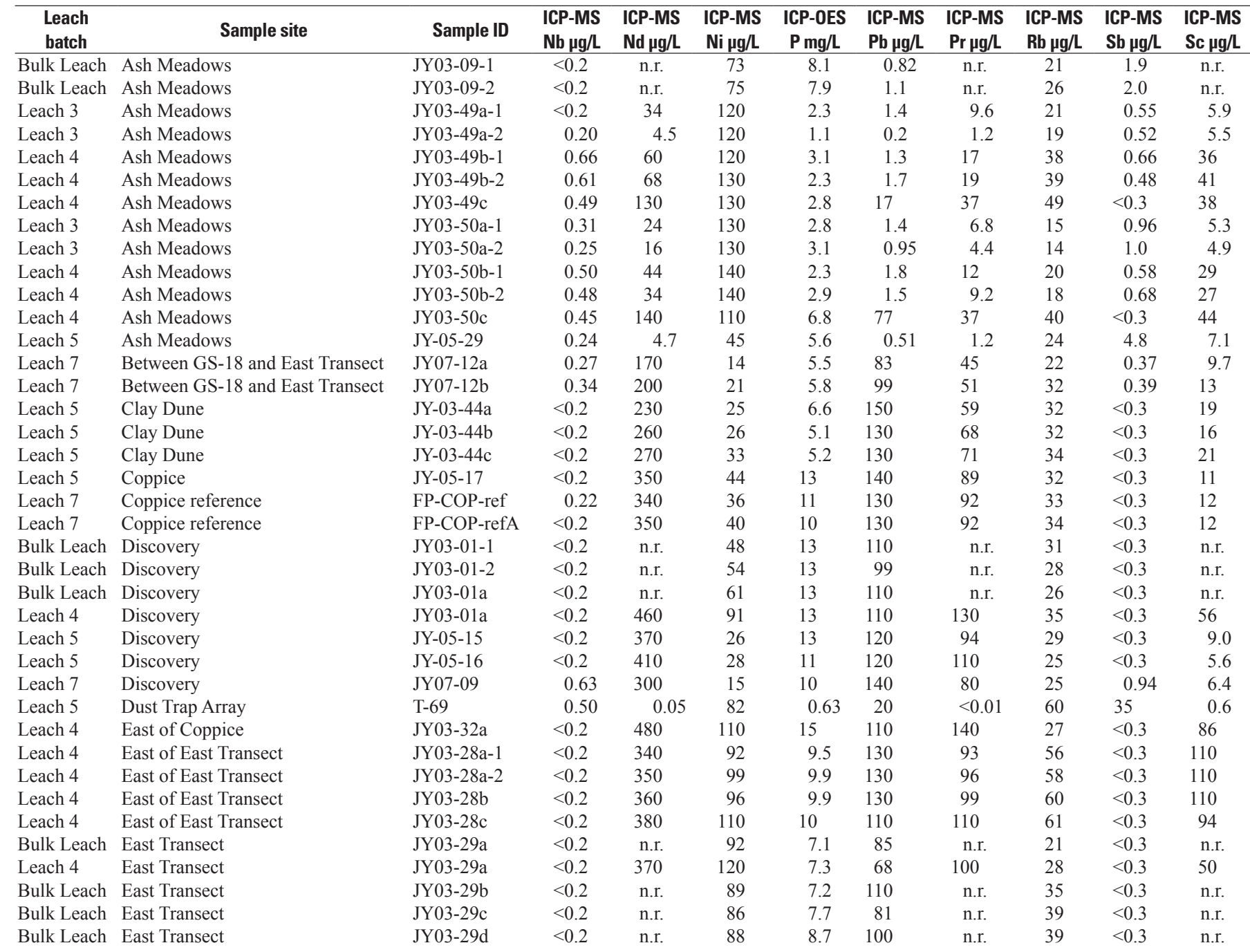


Table 12E. Composition of the nitric acid leach bulk grab samples. Method of determination (ICP-MS, inductively coupled plasmamass spectrometry; ICP-OES, inductively coupled plasma-optical emission spectrometry) is listed above each element.-Continued

[Leach batch, denotes the batch in which the sample was processed and analyzed; Sample site, name of sampling site; Sample ID, unique sample identifier; $-1,-2$, denotes duplicates; BC, bag contents-hydrated salts converted to liquid upon return from field; d, duplicate; n.r., not reported; ins, insufficient sample; $\mu \mathrm{g} / \mathrm{L}$, micrograms per liter; $\mathrm{mg} / \mathrm{L}$, milligrams per liter; $\mathrm{mg} / \mathrm{L} \mathrm{CaCO}$, milligrams per liter calcium carbonate $]$

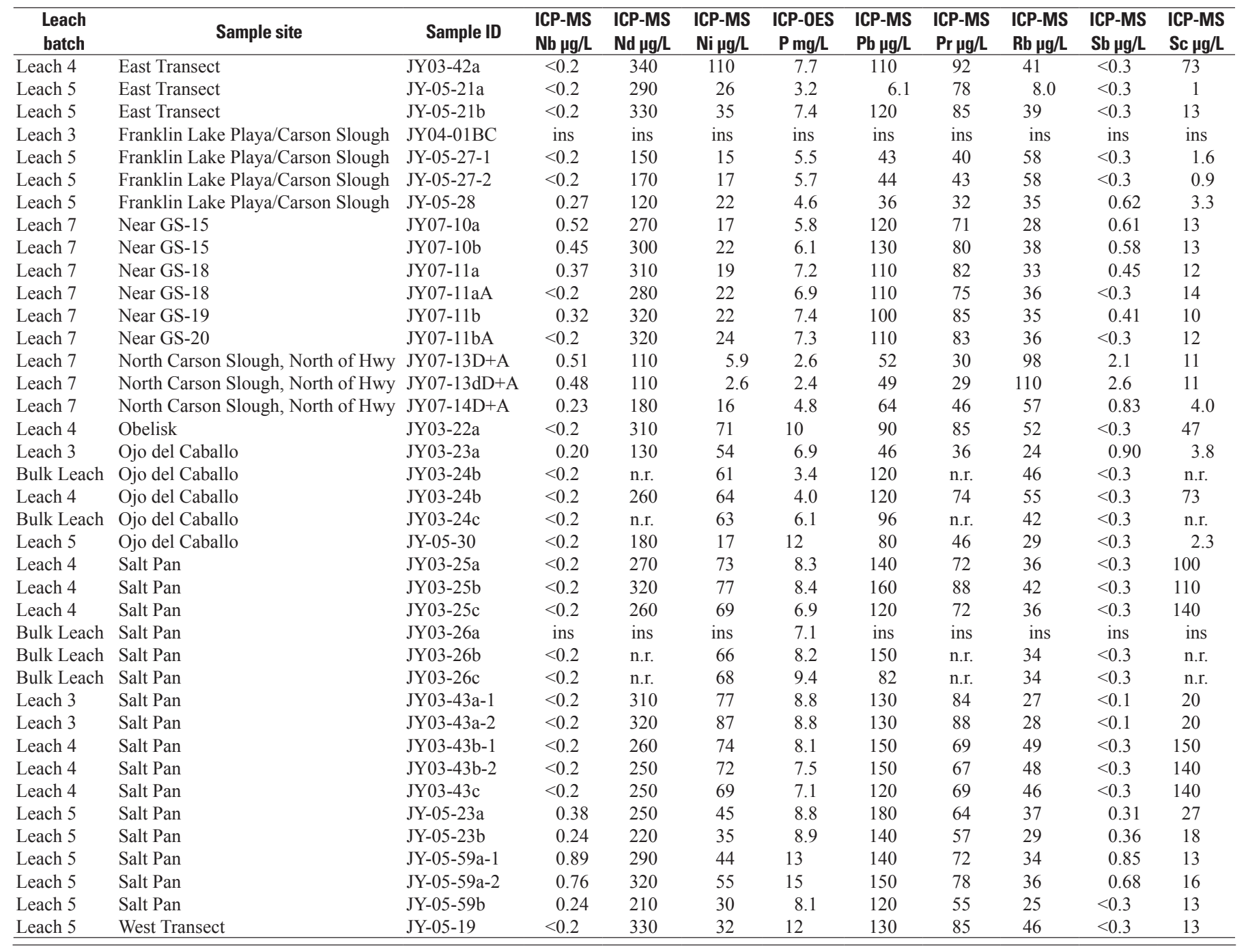


Table 12E. Composition of the nitric acid leach bulk grab samples. Method of determination (ICP-MS, inductively coupled plasmamass spectrometry; ICP-OES, inductively coupled plasma-optical emission spectrometry) is listed above each element.-Continued

[Leach batch, denotes the batch in which the sample was processed and analyzed; Sample site, name of sampling site; Sample ID, unique sample identifier; $-1,-2$, denotes duplicates; BC, bag contents-hydrated salts converted to liquid upon return from field; d, duplicate; n.r., not reported; ins, insufficient sample; $\mu \mathrm{g} / \mathrm{L}$, micrograms per liter; $\mathrm{mg} / \mathrm{L}$, milligrams per liter; $\mathrm{mg} / \mathrm{L} \mathrm{CaCO}_{3}$, milligrams per liter calcium carbonate]

\begin{tabular}{|c|c|c|c|c|c|c|c|c|c|c|c|}
\hline $\begin{array}{l}\text { Leach } \\
\text { batch }\end{array}$ & Sample site & Sample ID & $\begin{array}{l}\text { ICP-MS } \\
\mathrm{Nb} \mu \mathrm{g} / \mathrm{L}\end{array}$ & $\begin{array}{l}\text { ICP-MS } \\
\text { Nd } \mu \mathrm{g} / \mathrm{L}\end{array}$ & $\begin{array}{l}\text { ICP-MS } \\
\mathrm{Ni} \mu \mathrm{g} / \mathrm{L}\end{array}$ & $\begin{array}{c}\text { ICP-OES } \\
\text { P mg/L }\end{array}$ & $\begin{array}{l}\text { ICP-MS } \\
\mathrm{Pb} \mu \mathrm{g} / \mathrm{L}\end{array}$ & $\begin{array}{l}\text { ICP-MS } \\
\text { Pr } \mu g / L\end{array}$ & $\begin{array}{l}\text { ICP-MS } \\
\mathrm{Rb} \mu \mathrm{g} / \mathrm{L}\end{array}$ & $\begin{array}{l}\text { ICP-MS } \\
\text { Sb } \mu g / L\end{array}$ & $\begin{array}{l}\text { ICP-MS } \\
\text { Sc } \mu \mathrm{g} / \mathrm{L}\end{array}$ \\
\hline Bulk Leach & Ash Meadows & JY03-09-2 & $<0.2$ & n.r. & 75 & 7.9 & 1.1 & n.r. & 26 & 2.0 & n.r. \\
\hline Leach 3 & Ash Meadows & JY03-49a-1 & $<0.2$ & 34 & 120 & 2.3 & 1.4 & 9.6 & 21 & 0.55 & 5.9 \\
\hline Leach 4 & Ash Meadows & JY03-49b-1 & 0.66 & 60 & 120 & 3.1 & 1.3 & 17 & 38 & 0.66 & 36 \\
\hline Leach 4 & Ash Meadows & JY03-49b-2 & 0.61 & 68 & 130 & 2.3 & 1.7 & 19 & 39 & 0.48 & 41 \\
\hline Leach 4 & Ash Meadows & JY03-49c & 0.49 & 130 & 130 & 2.8 & 17 & 37 & 49 & $<0.3$ & 38 \\
\hline Leach 4 & Ash Meadows & JY03-50b-1 & 0.50 & 44 & 140 & 2.3 & 1.8 & 12 & 20 & 0.58 & 29 \\
\hline Leach 4 & Ash Meadows & JY03-50b-2 & 0.48 & 34 & 140 & 2.9 & 1.5 & 9.2 & 18 & 0.68 & 27 \\
\hline Leach 4 & Ash Meadows & JY03-50c & 0.45 & 140 & 110 & 6.8 & 77 & 37 & 40 & $<0.3$ & 44 \\
\hline Leach 5 & Ash Meadows & JY-05-29 & 0.24 & 4.7 & 45 & 5.6 & 0.51 & 1.2 & 24 & 4.8 & 7.1 \\
\hline Leach 7 & Between GS-18 and East Transect & JY07-12a & 0.27 & 170 & 14 & 5.5 & 83 & 45 & 22 & 0.37 & 9.7 \\
\hline Leach 7 & Between GS-18 and East Transect & JY07-12b & 0.34 & 200 & 21 & 5.8 & 99 & 51 & 32 & 0.39 & 13 \\
\hline Leach 7 & Coppice reference & FP-COP-refA & $<0.2$ & 350 & 40 & 10 & 130 & 92 & 34 & $<0.3$ & 12 \\
\hline Bulk Leach & Discovery & JY03-01-1 & $<0.2$ & n.r. & 48 & 13 & 110 & n.r. & 31 & $<0.3$ & n.r. \\
\hline Bulk Leach & Discovery & JY03-01-2 & $<0.2$ & n.r. & 54 & 13 & 99 & n.r. & 28 & $<0.3$ & n.r. \\
\hline Bulk Leach & Discovery & JY03-01a & $<0.2$ & n.r. & 61 & 13 & 110 & n.r. & 26 & $<0.3$ & n.r. \\
\hline Leach 4 & Discovery & JY03-01a & $<0.2$ & 460 & 91 & 13 & 110 & 130 & 35 & $<0.3$ & 56 \\
\hline Leach 5 & Discovery & JY-05-15 & $<0.2$ & 370 & 26 & 13 & 120 & 94 & 29 & $<0.3$ & 9.0 \\
\hline Leach 5 & Discovery & JY-05-16 & $<0.2$ & 410 & 28 & 11 & 120 & 110 & 25 & $<0.3$ & 5.6 \\
\hline Leach 7 & Discovery & JY07-09 & 0.63 & 300 & 15 & 10 & 140 & 80 & 25 & 0.94 & 6.4 \\
\hline Leach 5 & Dust Trap Array & $\mathrm{T}-69$ & 0.50 & 0.05 & 82 & 0.63 & 20 & $<0.01$ & 60 & 35 & 0.6 \\
\hline Leach 4 & East of Coppice & JY03-32a & $<0.2$ & 480 & 110 & 15 & 110 & 140 & 27 & $<0.3$ & 86 \\
\hline Leach 4 & East of East Transect & JY03-28a-1 & $<0.2$ & 340 & 92 & 9.5 & 130 & 93 & 56 & $<0.3$ & 110 \\
\hline Leach 4 & East of East Transect & JY03-28a-2 & $<0.2$ & 350 & 99 & 9.9 & 130 & 96 & 58 & $<0.3$ & 110 \\
\hline Leach 4 & East of East Transect & JY03-28b & $<0.2$ & 360 & 96 & 9.9 & 130 & 99 & 60 & $<0.3$ & 110 \\
\hline
\end{tabular}


Table 12E. Composition of the nitric acid leach bulk grab samples. Method of determination (ICP-MS, inductively coupled plasmamass spectrometry; ICP-OES, inductively coupled plasma-optical emission spectrometry) is listed above each element.-Continued

[Leach batch, denotes the batch in which the sample was processed and analyzed; Sample site, name of sampling site; Sample ID, unique sample identifier; $-1,-2$, denotes duplicates; BC, bag contents-hydrated salts converted to liquid upon return from field; d, duplicate; n.r., not reported; ins, insufficient sample; $\mu \mathrm{g} / \mathrm{L}$, micrograms per liter; $\mathrm{mg} / \mathrm{L}$, milligrams per liter; $\mathrm{mg} / \mathrm{L} \mathrm{CaCO}_{3}$, milligrams per liter calcium carbonate]

\begin{tabular}{|c|c|c|c|c|c|c|c|c|c|c|c|}
\hline $\begin{array}{l}\text { Leach } \\
\text { batch }\end{array}$ & Sample site & Sample ID & $\begin{array}{l}\text { ICP-MS } \\
\mathrm{Nb} \mu \mathrm{g} / \mathrm{L}\end{array}$ & $\begin{array}{l}\text { ICP-MS } \\
\text { Nd } \mu g / L\end{array}$ & $\begin{array}{l}\text { ICP-MS } \\
\mathrm{Ni} \mu \mathrm{g} / \mathrm{L}\end{array}$ & $\begin{array}{c}\text { ICP-OES } \\
\text { P mg/L }\end{array}$ & $\begin{array}{l}\text { ICP-MS } \\
\mathrm{Pb} \mu \mathrm{g} / \mathrm{L}\end{array}$ & $\begin{array}{l}\text { ICP-MS } \\
\text { Pr } \mu g / L\end{array}$ & $\begin{array}{l}\text { ICP-MS } \\
\mathrm{Rb} \mu \mathrm{g} / \mathrm{L}\end{array}$ & $\begin{array}{l}\text { ICP-MS } \\
\text { Sb } \mu g / L\end{array}$ & $\begin{array}{l}\text { ICP-MS } \\
\text { Sc } \mu g / L\end{array}$ \\
\hline Leach 5 & East Transect & JY-05-21a & $<0.2$ & 290 & 26 & 3.2 & 6.1 & 78 & 8.0 & $<0.3$ & 1 \\
\hline Leach 5 & East Transect & JY-05-21b & $<0.2$ & 330 & 35 & 7.4 & 120 & 85 & 39 & $<0.3$ & 13 \\
\hline Leach 5 & Franklin Lake Playa/Carson Slough & JY-05-27-1 & $<0.2$ & 150 & 15 & 5.5 & 43 & 40 & 58 & $<0.3$ & 1.6 \\
\hline Leach 5 & Franklin Lake Playa/Carson Slough & JY-05-27-2 & $<0.2$ & 170 & 17 & 5.7 & 44 & 43 & 58 & $<0.3$ & 0.9 \\
\hline Leach 5 & Franklin Lake Playa/Carson Slough & JY-05-28 & 0.27 & 120 & 22 & 4.6 & 36 & 32 & 35 & 0.62 & 3.3 \\
\hline Leach 7 & Near GS-18 & JY07-11a & 0.37 & 310 & 19 & 7.2 & 110 & 82 & 33 & 0.45 & 12 \\
\hline Leach 7 & Near GS-18 & JY07-11aA & $<0.2$ & 280 & 22 & 6.9 & 110 & 75 & 36 & $<0.3$ & 14 \\
\hline Leach 7 & Near GS-19 & JY07-11b & 0.32 & 320 & 22 & 7.4 & 100 & 85 & 35 & 0.41 & 10 \\
\hline Leach 7 & Near GS-20 & JY07-11bA & $<0.2$ & 320 & 24 & 7.3 & 110 & 83 & 36 & $<0.3$ & 12 \\
\hline Leach 7 & North Carson Slough, North of Hwy & JY07-13D+A & 0.51 & 110 & 5.9 & 2.6 & 52 & 30 & 98 & 2.1 & 11 \\
\hline Leach 7 & North Carson Slough, North of Hwy & $\mathrm{JY} 07-13 \mathrm{dD}+\mathrm{A}$ & 0.48 & 110 & 2.6 & 2.4 & 49 & 29 & 110 & 2.6 & 11 \\
\hline Bulk Leach & Ojo del Caballo & JY03-24c & $<0.2$ & n.r. & 63 & 6.1 & 96 & n.r. & 42 & $<0.3$ & n.r. \\
\hline Leach 5 & Ojo del Caballo & JY-05-30 & $<0.2$ & 180 & 17 & 12 & 80 & 46 & 29 & $<0.3$ & 2.3 \\
\hline Leach 4 & Salt Pan & $J Y 03-25 a$ & $<0.2$ & 270 & 73 & 8.3 & 140 & 72 & 36 & $<0.3$ & 100 \\
\hline Leach 4 & Salt Pan & JY03-25b & $<0.2$ & 320 & 77 & 8.4 & 160 & 88 & 42 & $<0.3$ & 110 \\
\hline Leach 4 & Salt Pan & $J Y 03-25 c$ & $<0.2$ & 260 & 69 & 6.9 & 120 & 72 & 36 & $<0.3$ & 140 \\
\hline Bulk Leach & Salt Pan & JY03-26a & ins & ins & ins & 7.1 & ins & ins & ins & ins & ins \\
\hline Bulk Leach & Salt Pan & JY03-26b & $<0.2$ & n.r. & 66 & 8.2 & 150 & n.r. & 34 & $<0.3$ & n.r. \\
\hline Bulk Leach & Salt Pan & JY03-26c & $<0.2$ & n.r. & 68 & 9.4 & 82 & n.r. & 34 & $<0.3$ & n.r. \\
\hline Leach 3 & Salt Pan & JY03-43a-1 & $<0.2$ & 310 & 77 & 8.8 & 130 & 84 & 27 & $<0.1$ & 20 \\
\hline Leach 3 & Salt Pan & JY03-43a-2 & $<0.2$ & 320 & 87 & 8.8 & 130 & 88 & 28 & $<0.1$ & 20 \\
\hline Leach 4 & Salt Pan & JY03-43b-1 & $<0.2$ & 260 & 74 & 8.1 & 150 & 69 & 49 & $<0.3$ & 150 \\
\hline Leach 4 & Salt Pan & JY03-43b-2 & $<0.2$ & 250 & 72 & 7.5 & 150 & 67 & 48 & $<0.3$ & 140 \\
\hline Leach 4 & Salt Pan & JY03-43c & $<0.2$ & 250 & 69 & 7.1 & 120 & 69 & 46 & $<0.3$ & 140 \\
\hline
\end{tabular}


Table 12E. Composition of the nitric acid leach bulk grab samples. Method of determination (ICP-MS, inductively coupled plasmamass spectrometry; ICP-OES, inductively coupled plasma-optical emission spectrometry) is listed above each element.-Continued

[Leach batch, denotes the batch in which the sample was processed and analyzed; Sample site, name of sampling site; Sample ID, unique sample identifier; $-1,-2$, denotes duplicates; BC, bag contents-hydrated salts converted to liquid upon return from field; d, duplicate; n.r., not reported; ins, insufficient sample; $\mu \mathrm{g} / \mathrm{L}$, micrograms per liter; $\mathrm{mg} / \mathrm{L}$, milligrams per liter; $\mathrm{mg} / \mathrm{L} \mathrm{CaCO}_{3}$, milligrams per liter calcium carbonate]

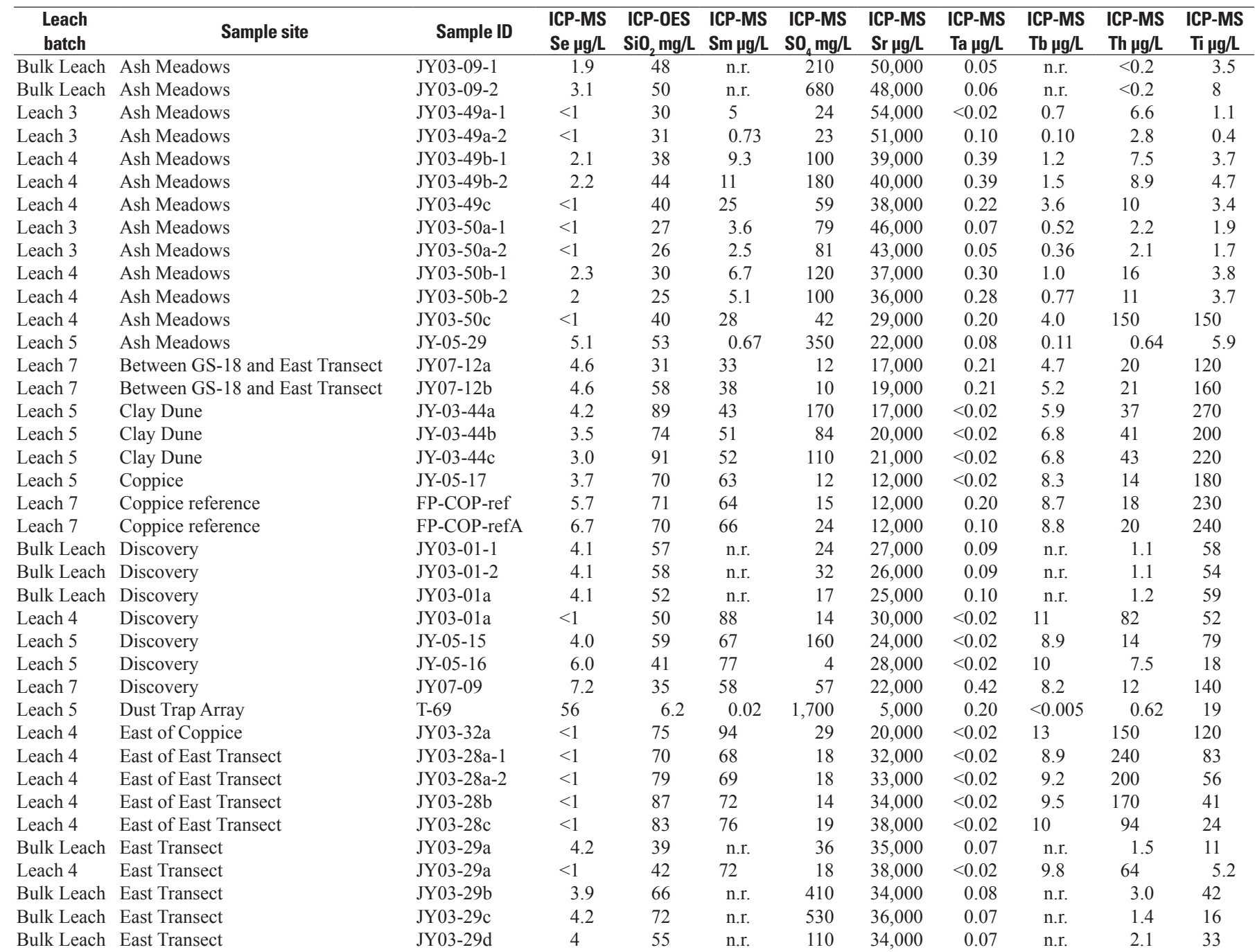


Table 12E. Composition of the nitric acid leach bulk grab samples. Method of determination (ICP-MS, inductively coupled plasmamass spectrometry; ICP-OES, inductively coupled plasma-optical emission spectrometry) is listed above each element.-Continued

[Leach batch, denotes the batch in which the sample was processed and analyzed; Sample site, name of sampling site; Sample ID, unique sample identifier; $-1,-2$, denotes duplicates; BC, bag contents-hydrated salts converted to liquid upon return from field; d, duplicate; n.r., not reported; ins, insufficient sample; $\mu \mathrm{g} / \mathrm{L}$, micrograms per liter; $\mathrm{mg} / \mathrm{L}$, milligrams per liter; $\mathrm{mg} / \mathrm{L} \mathrm{CaCO}$, milligrams per liter calcium carbonate]

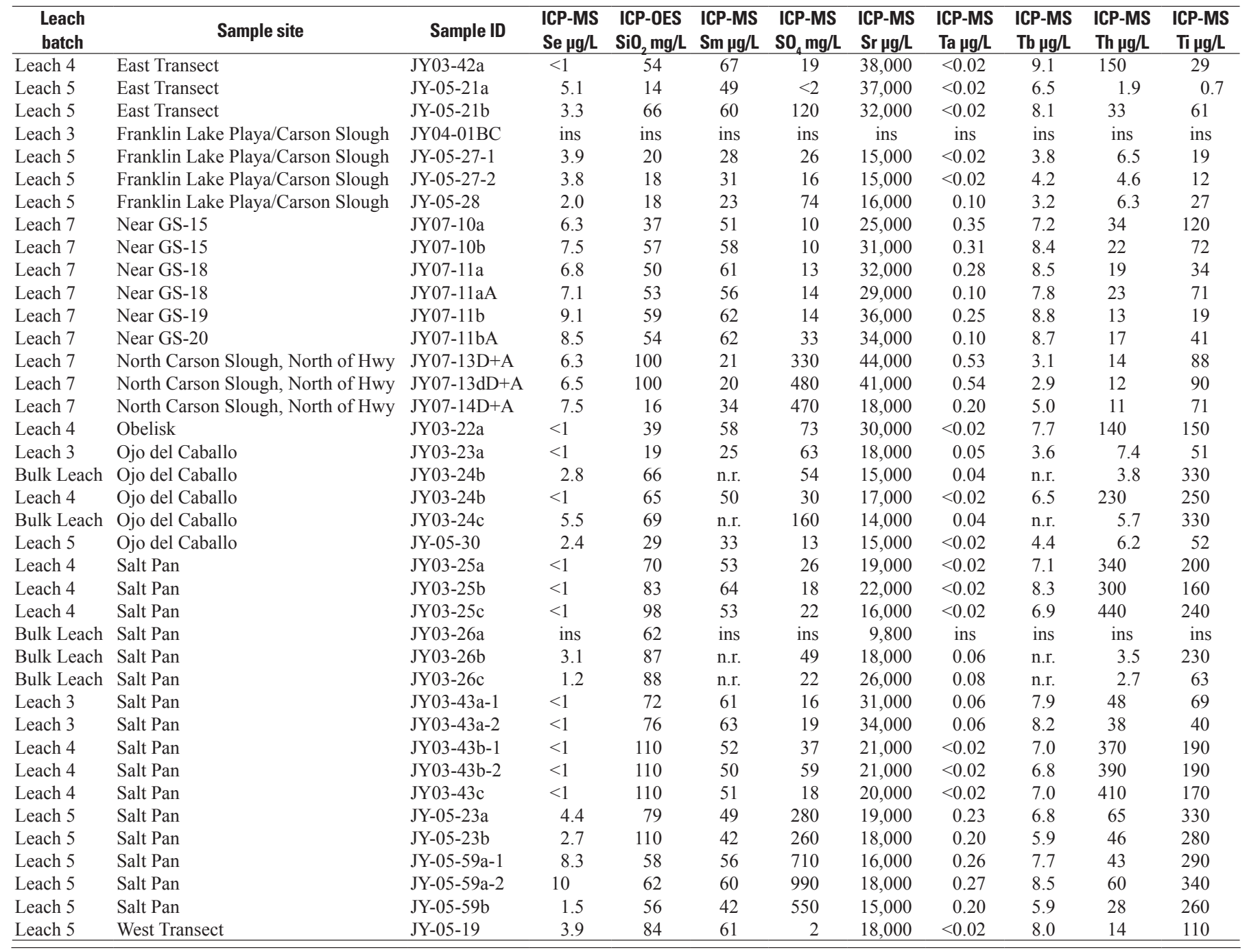


Table 12E. Composition of the nitric acid leach bulk grab samples. Method of determination (ICP-MS, inductively coupled plasmamass spectrometry; ICP-OES, inductively coupled plasma-optical emission spectrometry) is listed above each element.-Continued

[Leach batch, denotes the batch in which the sample was processed and analyzed; Sample site, name of sampling site; Sample ID, unique sample identifier; $-1,-2$, denotes duplicates; BC, bag contents-hydrated salts converted to liquid upon return from field; d, duplicate; n.r., not reported; ins, insufficient sample; $\mu \mathrm{g} / \mathrm{L}$, micrograms per liter; $\mathrm{mg} / \mathrm{L}$, milligrams per liter; $\mathrm{mg} / \mathrm{L} \mathrm{CaCO}_{3}$, milligrams per liter calcium carbonate]

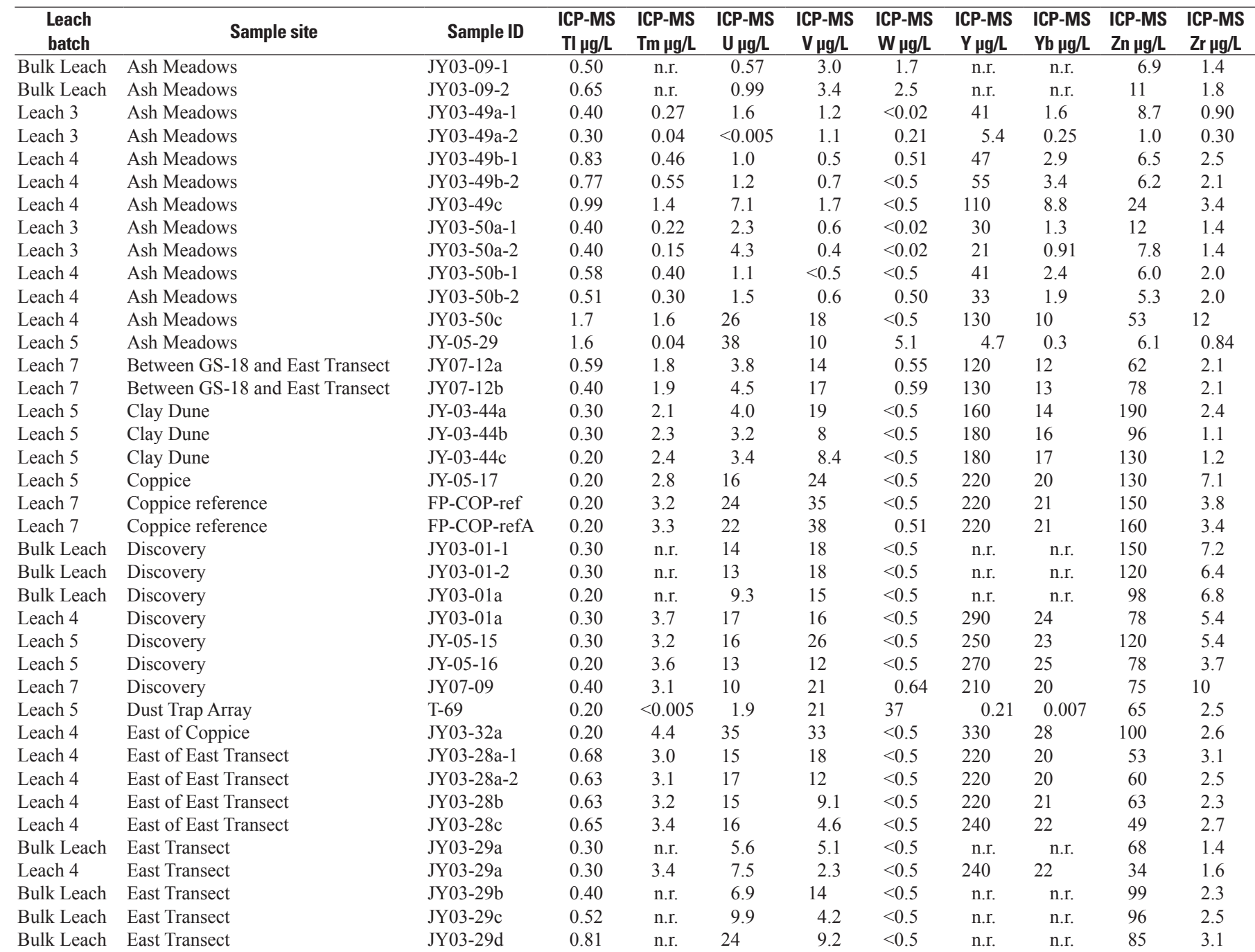


Table 12E. Composition of the nitric acid leach bulk grab samples. Method of determination (ICP-MS, inductively coupled plasmamass spectrometry; ICP-OES, inductively coupled plasma-optical emission spectrometry) is listed above each element.-Continued

[Leach batch, denotes the batch in which the sample was processed and analyzed; Sample site, name of sampling site; Sample ID, unique sample identifier; $-1,-2$, denotes duplicates; BC, bag contents-hydrated salts converted to liquid upon return from field; d, duplicate; n.r., not reported; ins, insufficient sample; $\mu \mathrm{g} / \mathrm{L}$, micrograms per liter; $\mathrm{mg} / \mathrm{L}$, milligrams per liter; $\mathrm{mg} / \mathrm{L} \mathrm{CaCO}_{3}$, milligrams per liter calcium carbonate]

\begin{tabular}{|c|c|c|c|c|c|c|c|c|c|c|c|}
\hline $\begin{array}{l}\text { Leach } \\
\text { batch }\end{array}$ & Sample site & Sample ID & $\begin{array}{c}\text { ICP-MS } \\
\text { TI } \mu \mathrm{g} / \mathrm{L}\end{array}$ & $\begin{array}{l}\text { ICP-MS } \\
\mathrm{Tm} \mu \mathrm{g} / \mathrm{L}\end{array}$ & $\begin{array}{c}\text { ICP-MS } \\
\mathrm{U} \mu \mathrm{g} / \mathrm{L}\end{array}$ & $\begin{array}{c}\text { ICP-MS } \\
\text { V } \mu \mathrm{g} / \mathrm{L}\end{array}$ & $\begin{array}{l}\text { ICP-MS } \\
W \mu g / L\end{array}$ & $\begin{array}{c}\text { ICP-MS } \\
Y \mu g / L\end{array}$ & $\begin{array}{l}\text { ICP-MS } \\
\mathrm{Yb} \mu \mathrm{g} / \mathrm{L}\end{array}$ & $\begin{array}{l}\text { ICP-MS } \\
\mathrm{Zn} \mu \mathrm{g} / \mathrm{L}\end{array}$ & $\begin{array}{c}\text { ICP-MS } \\
\mathrm{Zr} \mu \mathrm{L} / \mathrm{L}\end{array}$ \\
\hline Leach 5 & East Transect & JY-05-21a & 0.20 & 2.3 & 0.68 & $<0.5$ & $<0.5$ & 180 & 15 & 7.5 & 0.65 \\
\hline Leach 5 & East Transect & $J Y-05-21 b$ & 0.62 & 2.8 & 6.7 & 22 & $<0.5$ & 200 & 20 & 73 & 2.3 \\
\hline Leach 5 & Franklin Lake Playa/Carson Slough & JY-05-27-1 & 0.78 & 1.2 & 12 & 17 & $<0.5$ & 92 & 7.8 & 130 & 7.7 \\
\hline Leach 5 & Franklin Lake Playa/Carson Slough & JY-05-27-2 & 2.1 & 1.6 & 11 & 15 & $<0.5$ & 120 & 11 & 45 & 5.0 \\
\hline Leach 5 & Franklin Lake Playa/Carson Slough & JY-05-28 & 0.88 & 1.2 & 9.6 & 12 & 0.89 & 91 & 7.5 & 43 & 6.8 \\
\hline Leach 7 & Near GS-18 & JY07-11a & 0.57 & 3.1 & 7.1 & 13 & $<0.5$ & 220 & 22 & 63 & 2.7 \\
\hline Leach 7 & Near GS-18 & JY07-11aA & 0.63 & 2.9 & 6.4 & 21 & $<0.5$ & 200 & 20 & 62 & 2.0 \\
\hline Leach 7 & Near GS-19 & JY07-11b & 0.50 & 3.3 & 6.9 & 6.5 & $<0.5$ & 230 & 22 & 62 & 2.6 \\
\hline Leach 7 & Near GS-20 & JY07-11bA & 0.62 & 3.2 & 6.3 & 18 & $<0.5$ & 220 & 21 & 65 & 2.6 \\
\hline Leach 7 & North Carson Slough, North of Hwy & $J Y 07-13 D+A$ & 1.7 & 1.4 & 28 & 14 & 2.2 & 93 & 8.8 & 58 & 13 \\
\hline Leach 7 & North Carson Slough, North of Hwy & JY07-13dD+A & 1.8 & 1.3 & 33 & 14 & 1.4 & 88 & 8.6 & 52 & 13 \\
\hline Bulk Leach & Ojo del Caballo & JY03-24c & 0.50 & n.r. & 33 & 63 & $<0.5$ & n.r. & n.r. & 190 & 1.2 \\
\hline Leach 5 & Ojo del Caballo & JY-05-30 & 0.60 & 1.5 & 12 & 36 & $<0.5$ & 110 & 11 & 53 & 4.1 \\
\hline Leach 4 & Salt Pan & JY03-25a & 0.40 & 2.3 & 7.8 & 15 & $<0.5$ & 180 & 15 & 93 & 1.6 \\
\hline Leach 4 & Salt Pan & JY03-25b & 0.40 & 2.6 & 7.9 & 14 & $<0.5$ & 200 & 17 & 91 & 1.5 \\
\hline Leach 4 & Salt Pan & $J Y 03-25 c$ & 0.30 & 2.3 & 5.4 & 14 & $<0.5$ & 170 & 16 & 98 & 1.0 \\
\hline Bulk Leach & Salt Pan & JY03-26a & ins & ins & ins & ins & ins & ins & ins & ins & ins \\
\hline Bulk Leach & Salt Pan & JY03-26b & 0.30 & n.r. & 4.3 & 18 & $<0.5$ & n.r. & n.r. & 160 & 1.7 \\
\hline Bulk Leach & Salt Pan & JY03-26c & 0.40 & n.r. & 6.2 & 7.1 & $<0.5$ & n.r. & n.r. & 94 & 1.1 \\
\hline Leach 3 & Salt Pan & JY03-43a-1 & 0.30 & 2.9 & 5.5 & 5.7 & $<0.02$ & 300 & 19 & 100 & 0.59 \\
\hline Leach 3 & Salt Pan & JY03-43a-2 & 0.20 & 3.0 & 5.8 & 3.6 & $<0.02$ & 330 & 20 & 100 & 0.60 \\
\hline Leach 4 & Salt Pan & JY03-43b-1 & 0.40 & 2.2 & 9.9 & 9.7 & $<0.5$ & 180 & 15 & 95 & 1.3 \\
\hline Leach 4 & Salt Pan & JY03-43b-2 & 0.40 & 2.2 & 9.1 & 10 & $<0.5$ & 180 & 14 & 89 & 1.4 \\
\hline Leach 4 & Salt Pan & JY03-43c & 0.50 & 2.2 & 7.7 & 7.7 & $<0.5$ & 180 & 15 & 81 & 0.98 \\
\hline
\end{tabular}


Table 12F. Composition of the residual fraction of bulk grab samples. Method of determination (ICP-MS, inductively coupled plasmamass spectrometry; ICP-OES, inductively coupled plasma-optical emission spectrometry) is listed above each element.

[Leach batch, denotes the batch in which the sample was processed and analyzed; Sample site, name of sampling site; Sample ID, unique sample identifier; $-1,-2$, denotes duplicates; BC, bag contents-hydrated salts converted to liquid upon return from field; n.d., not determined; ins, insufficient sample; ppm, parts per million; wt.\%, weight percent]

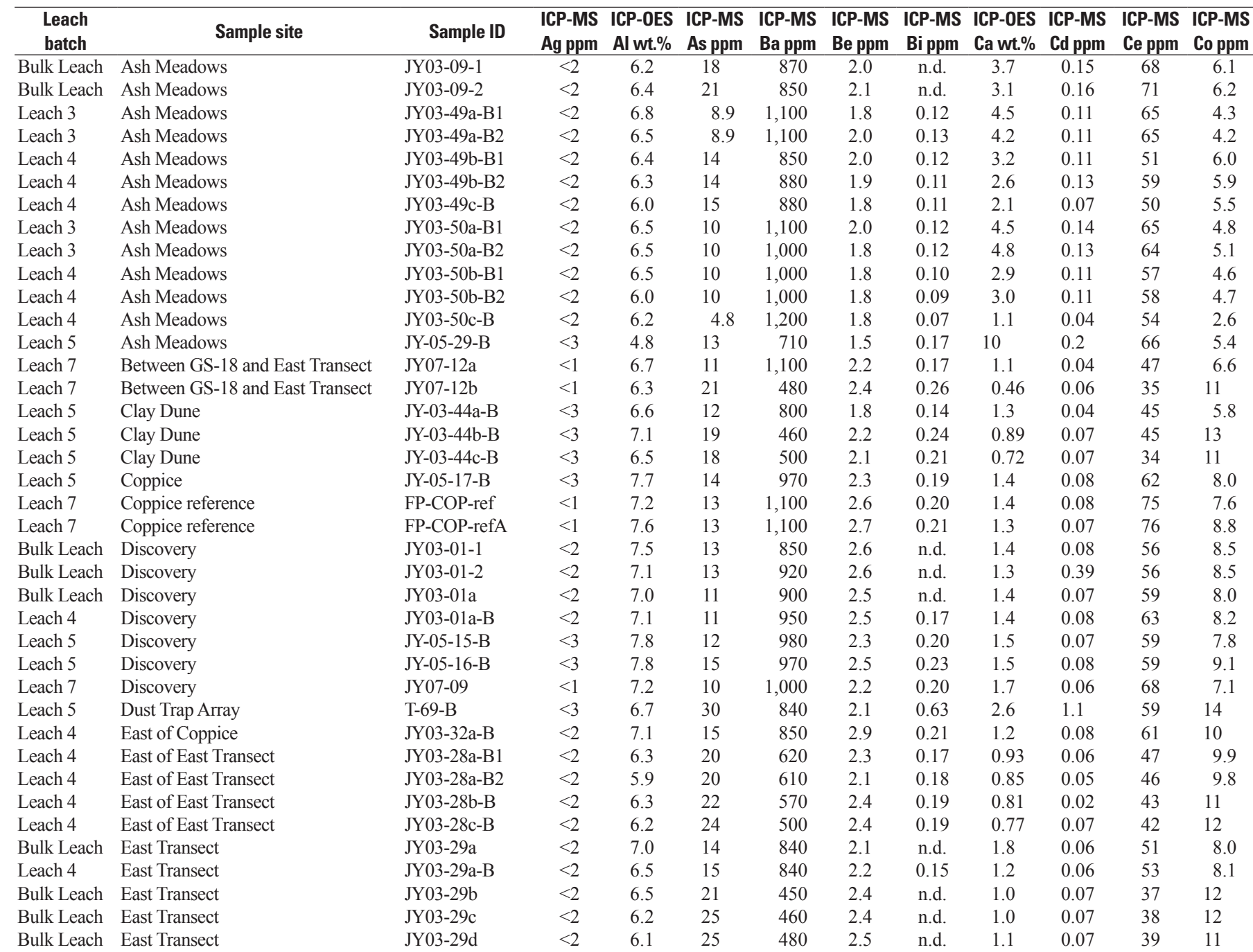


Table 12F. Composition of the residual fraction of bulk grab samples. Method of determination (ICP-MS, inductively coupled plasmamass spectrometry; ICP-OES, inductively coupled plasma-optical emission spectrometry) is listed above each element.-Continued

[Leach batch, denotes the batch in which the sample was processed and analyzed; Sample site, name of sampling site; Sample ID, unique sample identifier; $-1,-2$, denotes duplicates; BC, bag contents-hydrated salts converted to liquid upon return from field; n.d., not determined; ins, insufficient sample; ppm, parts per million; wt.\%, weight percent]

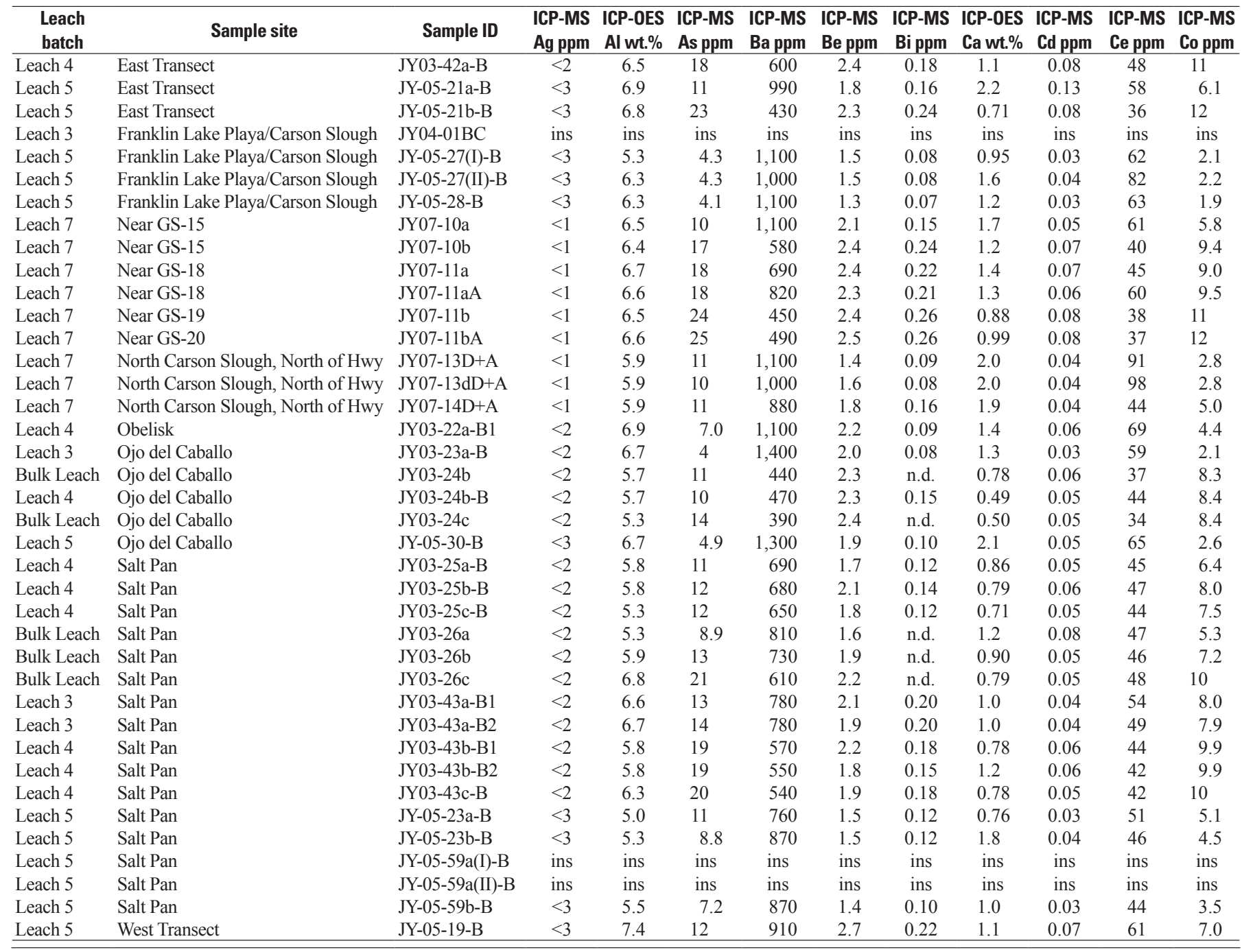


Table 12F. Composition of the residual fraction of bulk grab samples. Method of determination (ICP-MS, inductively coupled plasmamass spectrometry; ICP-OES, inductively coupled plasma-optical emission spectrometry) is listed above each element.-Continued

[Leach batch, denotes the batch in which the sample was processed and analyzed; Sample site, name of sampling site; Sample ID, unique sample identifier; $-1,-2$, denotes duplicates; BC, bag contents-hydrated salts converted to liquid upon return from field; n.d., not determined; ins, insufficient sample; ppm, parts per million; wt.\%, weight percent]

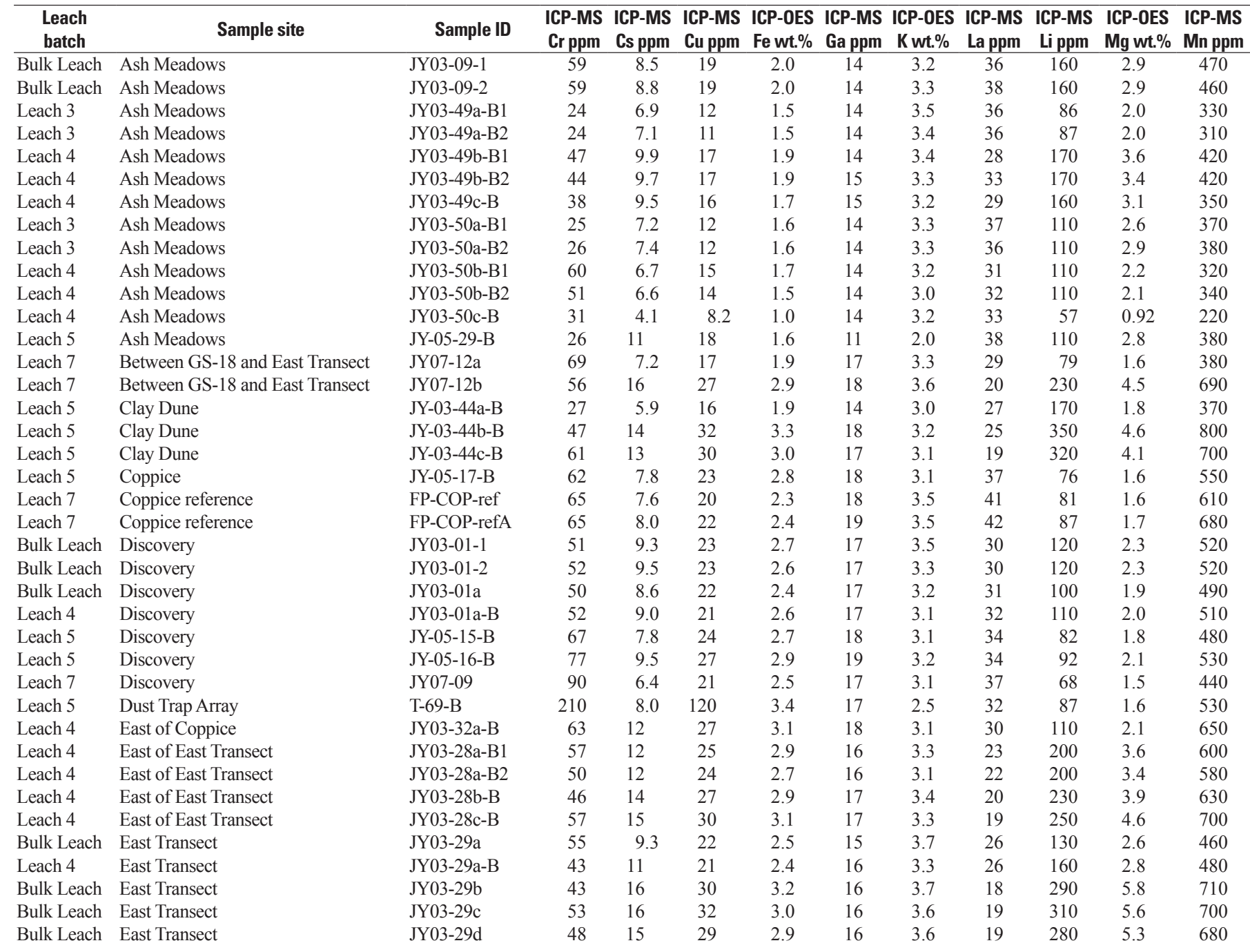


Table 12F. Composition of the residual fraction of bulk grab samples. Method of determination (ICP-MS, inductively coupled plasmamass spectrometry; ICP-OES, inductively coupled plasma-optical emission spectrometry) is listed above each element.-Continued

[Leach batch, denotes the batch in which the sample was processed and analyzed; Sample site, name of sampling site; Sample ID, unique sample identifier; $-1,-2$, denotes duplicates; BC, bag contents-hydrated salts converted to liquid upon return from field; n.d., not determined; ins, insufficient sample; ppm, parts per million; wt.\%, weight percent]

\begin{tabular}{|c|c|c|c|c|c|c|c|c|c|c|c|c|}
\hline $\begin{array}{l}\text { Leach } \\
\text { batch }\end{array}$ & Sample site & Sample ID & $\begin{array}{l}\text { ICP-MS } \\
\text { Cr ppm }\end{array}$ & $\begin{array}{l}\text { ICP-MS } \\
\text { Cs ppm }\end{array}$ & $\begin{array}{l}\text { ICP-MS } \\
\text { Cu ppm }\end{array}$ & $\begin{array}{l}\text { ICP-0ES } \\
\text { Fe wt.\% }\end{array}$ & $\begin{array}{l}\text { ICP-MS } \\
\text { Ga ppm }\end{array}$ & $\begin{array}{l}\text { ICP-OES } \\
\text { K wt. \% }\end{array}$ & $\begin{array}{l}\text { ICP-MS } \\
\text { La ppm }\end{array}$ & $\begin{array}{l}\text { ICP-MS } \\
\text { Li ppm }\end{array}$ & $\begin{array}{l}\text { ICP-OES } \\
\text { Mg wt.\% }\end{array}$ & $\begin{array}{l}\text { ICP-MS } \\
\text { Mn ppm }\end{array}$ \\
\hline Leach 5 & East Transect & JY-05-21a-B & 72 & 5.8 & 23 & 2.1 & 15 & 3.0 & 32 & 55 & 1.5 & 350 \\
\hline Leach 5 & East Transect & JY-05-21b-B & 48 & 16 & 31 & 3.1 & 17 & 3.5 & 19 & 270 & 5.4 & 720 \\
\hline Leach 5 & Franklin Lake Playa/Carson Slough & JY-05-27(I)-B & 12 & 2.0 & 6.2 & 0.85 & 13 & 2.5 & 39 & 53 & 0.37 & 210 \\
\hline Leach 5 & Franklin Lake Playa/Carson Slough & JY-05-27(II)-B & 13 & 2.1 & 6.6 & 0.95 & 13 & 2.9 & 50 & 55 & 0.43 & 220 \\
\hline Leach 5 & Franklin Lake Playa/Carson Slough & JY-05-28-B & 11 & 1.8 & 4.8 & 0.85 & 14 & 2.9 & 40 & 38 & 0.39 & 180 \\
\hline Leach 7 & Near GS-18 & JY07-11a & 57 & 12 & 23 & 2.5 & 18 & 3.6 & 26 & 170 & 3.6 & 600 \\
\hline Leach 7 & Near GS-18 & JY07-11aA & 61 & 12 & 24 & 2.4 & 18 & 3.5 & 32 & 170 & 3.5 & 640 \\
\hline Leach 7 & Near GS-19 & JY07-11b & 51 & 17 & 28 & 2.9 & 18 & 3.7 & 21 & 240 & 5.0 & 730 \\
\hline Leach 7 & Near GS-20 & JY07-11bA & 62 & 16 & 30 & 2.8 & 19 & 3.6 & 21 & 240 & 4.9 & 790 \\
\hline Leach 7 & North Carson Slough, North of Hwy & JY07-13D+A & 88 & 2.1 & 13 & 1.3 & 14 & 3.0 & 53 & 94 & 1.9 & 280 \\
\hline Leach 7 & North Carson Slough, North of Hwy & JY07-13dD+A & 91 & 2.1 & 13 & 1.3 & 14 & 3.0 & 65 & 96 & 2.0 & 280 \\
\hline Bulk Leach & Ojo del Caballo & JY03-24c & 34 & 14 & 23 & 2.5 & 14 & 3.8 & 19 & 240 & 5.0 & 600 \\
\hline Leach 5 & Ojo del Caballo & JY-05-30-B & 17 & 2.3 & 7.4 & 1.1 & 15 & 2.9 & 40 & 29 & 0.52 & 240 \\
\hline Leach 4 & Salt Pan & JY03-25a-B & 41 & 7.1 & 17 & 2.2 & 13 & 3.0 & 22 & 140 & 2.1 & 400 \\
\hline Leach 4 & Salt Pan & JY03-25b-B & 48 & 9.6 & 21 & 2.4 & 15 & 3.0 & 23 & 190 & 2.3 & 470 \\
\hline Leach 4 & Salt Pan & JY03-25c-B & 44 & 8.5 & 20 & 2.2 & 13 & 2.8 & 22 & 230 & 2.3 & 460 \\
\hline Bulk Leach & Salt Pan & JY03-26a & 170 & 3.6 & 29 & 2.1 & 12 & 2.6 & 25 & 84 & 0.94 & 310 \\
\hline Bulk Leach & Salt Pan & JY03-26b & 49 & 7.2 & 19 & 2.2 & 14 & 3.1 & 24 & 220 & 2.0 & 440 \\
\hline Bulk Leach & Salt Pan & JY03-26c & 50 & 11 & 25 & 2.9 & 16 & 3.8 & 25 & 280 & 2.9 & 610 \\
\hline Leach 3 & Salt Pan & JY03-43a-B1 & 36 & 8.2 & 18 & 2.4 & 16 & 3.5 & 29 & 150 & 2.1 & 470 \\
\hline Leach 3 & Salt Pan & JY03-43a-B2 & 35 & 8.4 & 18 & 2.4 & 16 & 3.5 & 26 & 150 & 2.3 & 470 \\
\hline Leach 4 & Salt Pan & JY03-43b-B1 & 46 & 12 & 25 & 2.8 & 15 & 3.2 & 21 & 470 & 3.7 & 610 \\
\hline Leach 4 & Salt Pan & JY03-43b-B2 & 45 & 11 & 25 & 2.7 & 16 & 3.2 & 22 & 470 & 3.7 & 640 \\
\hline Leach 4 & Salt Pan & JY03-43c-B & 55 & 11 & 26 & 3.0 & 16 & 3.5 & 22 & 460 & 3.8 & 640 \\
\hline
\end{tabular}


Table 12F. Composition of the residual fraction of bulk grab samples. Method of determination (ICP-MS, inductively coupled plasmamass spectrometry; ICP-OES, inductively coupled plasma-optical emission spectrometry) is listed above each element.-Continued

[Leach batch, denotes the batch in which the sample was processed and analyzed; Sample site, name of sampling site; Sample ID, unique sample identifier; $-1,-2$, denotes duplicates; BC, bag contents-hydrated salts converted to liquid upon return from field; n.d., not determined; ins, insufficient sample; ppm, parts per million; wt.\%, weight percent]

\begin{tabular}{|c|c|c|c|c|c|c|c|c|c|c|c|}
\hline $\begin{array}{l}\text { Leach } \\
\text { batch }\end{array}$ & Sample site & Sample ID & $\begin{array}{l}\text { ICP-MS } \\
\text { Mo ppm }\end{array}$ & $\begin{array}{l}\text { ICP-OES } \\
\text { Na wt.\% }\end{array}$ & $\begin{array}{l}\text { ICP-MS } \\
\mathrm{Nb} \text { ppm }\end{array}$ & $\begin{array}{l}\text { ICP-OES } \\
\text { Nd ppm }\end{array}$ & $\begin{array}{l}\text { ICP-MS } \\
\text { Ni ppm }\end{array}$ & $\begin{array}{l}\text { ICP-MS } \\
\text { P ppm }\end{array}$ & $\begin{array}{l}\text { ICP-MS } \\
\text { Pb ppm }\end{array}$ & $\begin{array}{l}\text { ICP-MS } \\
\text { Rb ppm }\end{array}$ & $\begin{array}{l}\text { ICP-MS } \\
\text { Sb ppm }\end{array}$ \\
\hline Bulk Leach & Ash Meadows & JY03-09-2 & 2.0 & 1.7 & 20 & 31 & 13 & 720 & 19 & 120 & 1.0 \\
\hline Leach 3 & Ash Meadows & JY03-49a-B1 & 0.4 & 1.9 & 18 & 25 & 8.7 & 420 & 17 & 110 & 0.8 \\
\hline Leach 4 & Ash Meadows & JY03-49b-B1 & 0.9 & 1.5 & 11 & 19 & 12 & 370 & 16 & 120 & 0.9 \\
\hline Leach 4 & Ash Meadows & JY03-49b-B2 & 1.0 & 1.5 & 11 & 21 & 12 & 380 & 17 & 120 & 0.9 \\
\hline Leach 4 & Ash Meadows & JY03-49c-B & 1.1 & 1.5 & 11 & 18 & 11 & 360 & 15 & 120 & 0.8 \\
\hline Leach 4 & Ash Meadows & JY03-50b-B1 & 1.2 & 1.8 & 11 & 21 & 11 & 420 & 17 & 110 & 0.8 \\
\hline Leach 4 & Ash Meadows & JY03-50b-B2 & 1.0 & 1.7 & 12 & 20 & 10 & 380 & 17 & 110 & 0.7 \\
\hline Leach 4 & Ash Meadows & JY03-50c-B & 0.9 & 2.0 & 8.5 & 18 & 5.8 & 100 & 14 & 100 & 0.5 \\
\hline Leach 5 & Ash Meadows & JY-05-29-B & 2.0 & 1.1 & 12 & 26 & 11 & 660 & 18 & 91 & 1.1 \\
\hline Leach 7 & Between GS-18 and East Transect & JY07-12a & 1.0 & 1.8 & 20 & 19 & 14 & 110 & 15 & 130 & 1.0 \\
\hline Leach 7 & Between GS-18 and East Transect & JY07-12b & 0.8 & 0.81 & 23 & 12 & 23 & 170 & 14 & 180 & 1.7 \\
\hline Leach 7 & Coppice reference & FP-COP-refA & 2.3 & 2.0 & 28 & 26 & 21 & 280 & 21 & 150 & 1.5 \\
\hline Bulk Leach & Discovery & JY03-01-1 & 1.5 & 1.8 & 25 & 22 & 19 & 260 & 19 & 140 & 1.3 \\
\hline Bulk Leach & Discovery & JY03-01-2 & 1.4 & 1.7 & 27 & 20 & 19 & 260 & 19 & 140 & 1.3 \\
\hline Bulk Leach & Discovery & JY03-01a & 1.1 & 1.7 & 27 & 22 & 17 & 310 & 18 & 140 & 1.3 \\
\hline Leach 4 & Discovery & JY03-01a-B & 1.3 & 1.7 & 17 & 22 & 18 & 240 & 20 & 140 & 1.4 \\
\hline Leach 5 & Discovery & JY-05-15-B & 1.6 & 1.8 & 23 & 26 & 18 & 240 & 19 & 130 & 1.4 \\
\hline Leach 5 & Discovery & JY-05-16-B & 1.5 & 1.7 & 26 & 24 & 22 & 380 & 21 & 150 & 1.5 \\
\hline Leach 7 & Discovery & JY07-09 & 1.6 & 2.0 & 27 & 24 & 17 & 150 & 17 & 130 & 1.4 \\
\hline Leach 5 & Dust Trap Array & T-69-B & 20 & 1.3 & 21 & 25 & 140 & 1,300 & 320 & 120 & 14.0 \\
\hline Leach 4 & East of Coppice & JY03-32a-B & 2.6 & 1.6 & 19 & 20 & 27 & 230 & 22 & 150 & 1.6 \\
\hline Leach 4 & East of East Transect & JY03-28a-B1 & 1.0 & 1.1 & 15 & 16 & 21 & 290 & 16 & 150 & 1.4 \\
\hline Leach 4 & East of East Transect & JY03-28a-B2 & 0.9 & 1.0 & 13 & 15 & 20 & 270 & 15 & 140 & 1.5 \\
\hline Leach 4 & East of East Transect & JY03-28b-B & 0.9 & 0.93 & 15 & 14 & 22 & 300 & 16 & 160 & 1.6 \\
\hline
\end{tabular}


Table 12F. Composition of the residual fraction of bulk grab samples. Method of determination (ICP-MS, inductively coupled plasmamass spectrometry; ICP-OES, inductively coupled plasma-optical emission spectrometry) is listed above each element.-Continued

[Leach batch, denotes the batch in which the sample was processed and analyzed; Sample site, name of sampling site; Sample ID, unique sample identifier; $-1,-2$, denotes duplicates; BC, bag contents-hydrated salts converted to liquid upon return from field; n.d., not determined; ins, insufficient sample; ppm, parts per million; wt.\%, weight percent]

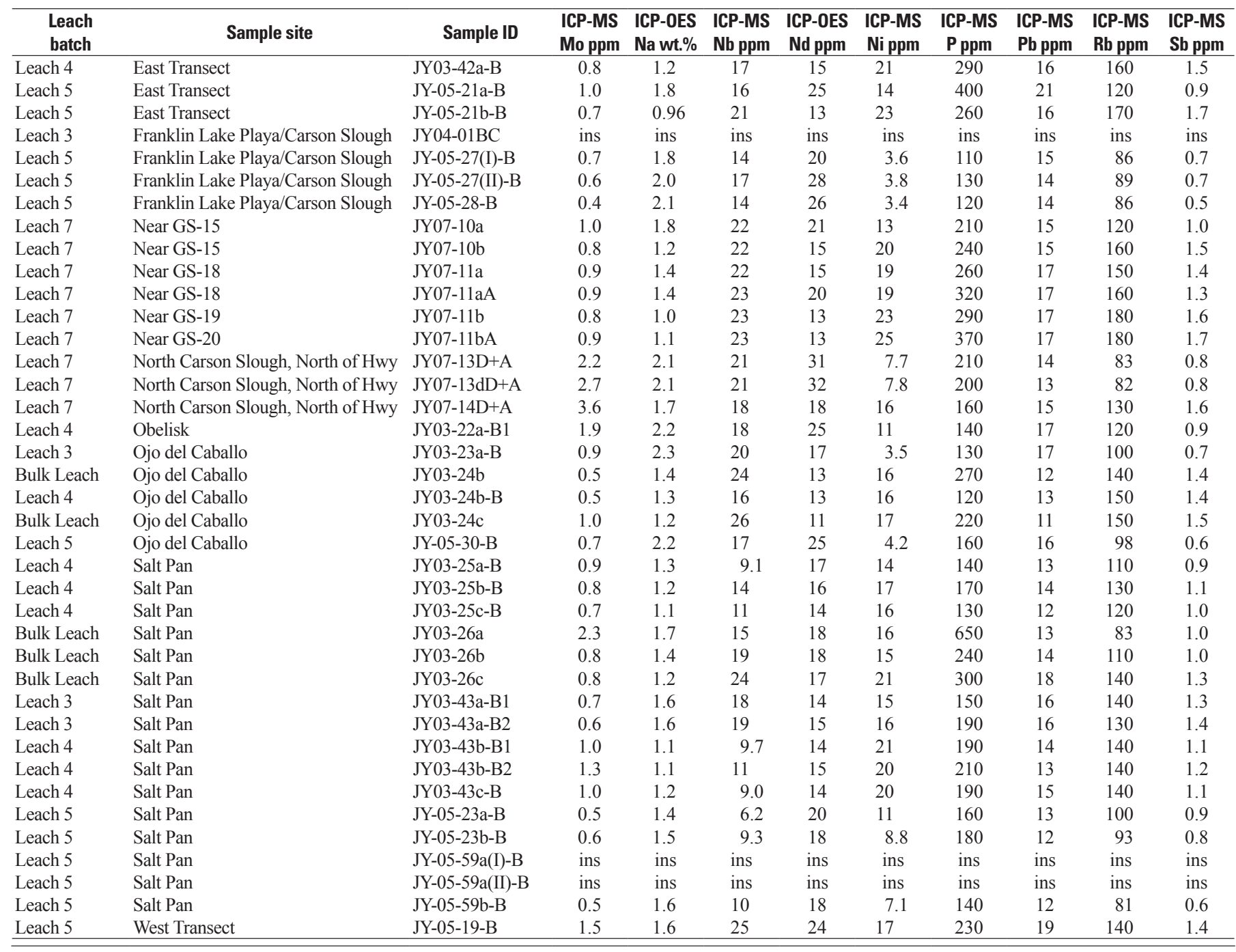


Table 12F. Composition of the residual fraction of bulk grab samples. Method of determination (ICP-MS, inductively coupled plasmamass spectrometry; ICP-OES, inductively coupled plasma-optical emission spectrometry) is listed above each element.-Continued

[Leach batch, denotes the batch in which the sample was processed and analyzed; Sample site, name of sampling site; Sample ID, unique sample identifier; $-1,-2$, denotes duplicates; BC, bag contents-hydrated salts converted to liquid upon return from field; n.d., not determined; ins, insufficient sample; ppm, parts per million; wt.\%, weight percent]

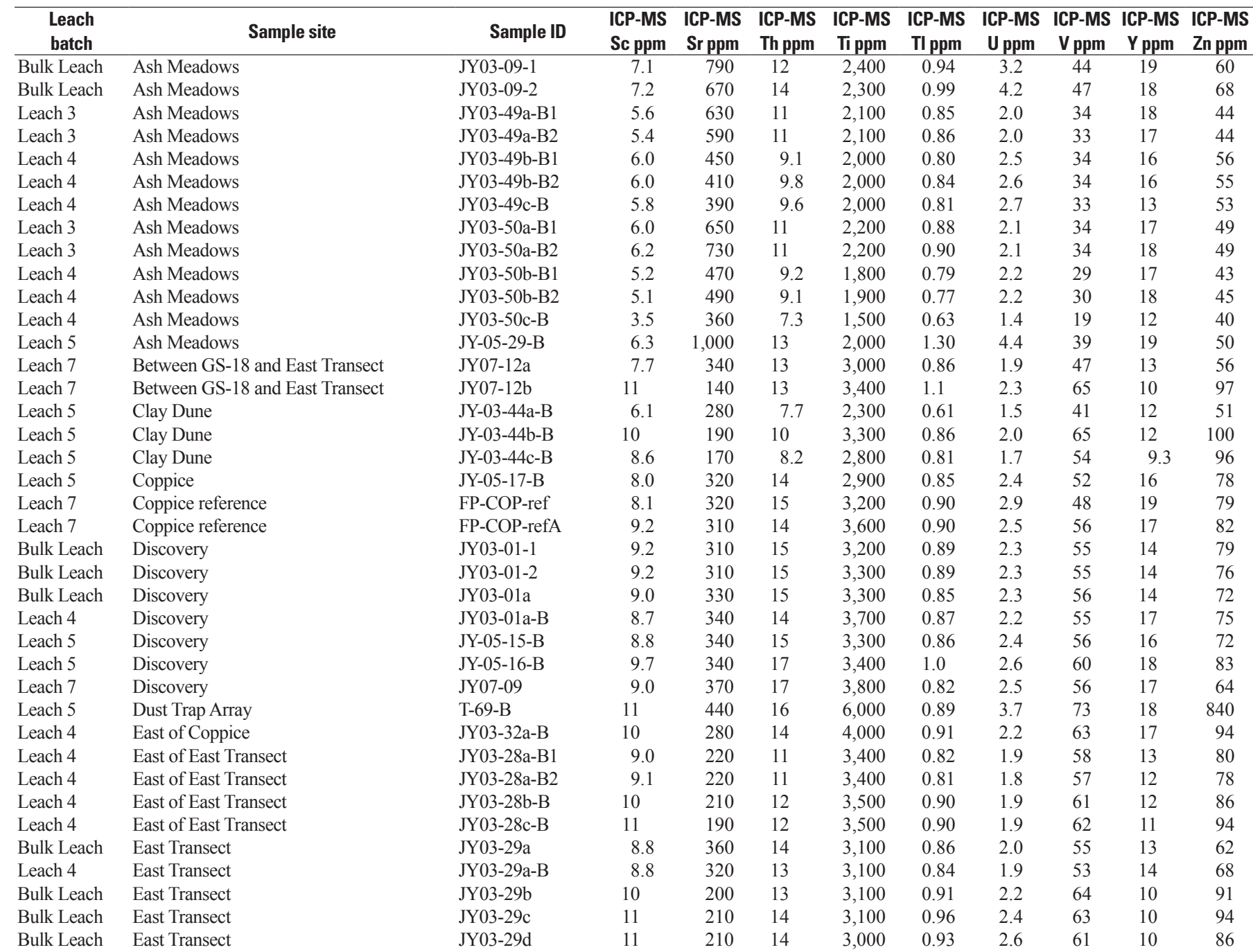


Table 12F. Composition of the residual fraction of bulk grab samples. Method of determination (ICP-MS, inductively coupled plasmamass spectrometry; ICP-OES, inductively coupled plasma-optical emission spectrometry) is listed above each element.-Continued

[Leach batch, denotes the batch in which the sample was processed and analyzed; Sample site, name of sampling site; Sample ID, unique sample identifier; $-1,-2$, denotes duplicates; BC, bag contents-hydrated salts converted to liquid upon return from field; n.d., not determined; ins, insufficient sample; ppm, parts per million; wt.\%, weight percent]

\begin{tabular}{|c|c|c|c|c|c|c|c|c|c|c|c|}
\hline $\begin{array}{l}\text { Leach } \\
\text { batch }\end{array}$ & Sample site & Sample ID & $\begin{array}{l}\text { ICP-MS } \\
\text { Sc ppm }\end{array}$ & $\begin{array}{l}\text { ICP-MS } \\
\text { Sr ppm }\end{array}$ & $\begin{array}{l}\text { ICP-MS } \\
\text { Th ppm }\end{array}$ & $\begin{array}{c}\text { ICP-MS } \\
\text { Ti ppm }\end{array}$ & $\begin{array}{l}\text { ICP-MS } \\
\text { TI ppm } \\
\end{array}$ & $\begin{array}{c}\text { ICP-MS } \\
\text { U ppm }\end{array}$ & $\begin{array}{c}\text { ICP-MS } \\
\text { V ppm }\end{array}$ & $\begin{array}{c}\text { ICP-MS } \\
\text { Y ppm }\end{array}$ & $\begin{array}{l}\text { ICP-MS } \\
\text { Zn ppm }\end{array}$ \\
\hline Leach 5 & East Transect & JY-05-21a-B & 7.2 & 390 & 16 & 2,500 & 0.84 & 2.0 & 46 & 16 & 53 \\
\hline Leach 5 & East Transect & JY-05-21b-B & 10 & 180 & 12 & 2,800 & 0.99 & 2.1 & 61 & 8.9 & 94 \\
\hline Leach 5 & Franklin Lake Playa/Carson Slough & JY-05-27(I)-B & 3.3 & 330 & 9.2 & 1,600 & 0.54 & 1.7 & 23 & 12 & 25 \\
\hline Leach 5 & Franklin Lake Playa/Carson Slough & JY-05-27(II)-B & 3.4 & 370 & 9.5 & 1,700 & 0.58 & 1.8 & 23 & 14 & 26 \\
\hline Leach 5 & Franklin Lake Playa/Carson Slough & JY-05-28-B & 3.0 & 360 & 8.5 & 1,500 & 0.54 & 1.7 & 18 & 13 & 23 \\
\hline Leach 7 & Near GS-18 & JY07-11a & 9.2 & 280 & 13 & 2,800 & 0.97 & 2.2 & 53 & 12 & 78 \\
\hline Leach 7 & Near GS-18 & JY07-11aA & 9.6 & 290 & 13 & 3,400 & 0.93 & 2.1 & 59 & 13 & 80 \\
\hline Leach 7 & Near GS-19 & JY07-11b & 11 & 200 & 14 & 3,100 & 1.1 & 2.5 & 62 & 11 & 96 \\
\hline Leach 7 & Near GS-20 & JY07-11bA & 12 & 210 & 14 & 3,400 & 1.1 & 2.3 & 66 & 11 & 100 \\
\hline Leach 7 & North Carson Slough, North of Hwy & JY07-13D+A & 4.4 & 430 & 11 & 2,400 & 0.55 & 1.6 & 26 & 16 & 31 \\
\hline Leach 7 & North Carson Slough, North of Hwy & JY07-13dD+A & 4.3 & 420 & 11 & 2,300 & 0.53 & 1.7 & 25 & 15 & 30 \\
\hline Bulk Leach & Ojo del Caballo & JY03-24c & 8.3 & 110 & 8.0 & 2,400 & 0.92 & 2.3 & 40 & 8.9 & 74 \\
\hline Leach 5 & Ojo del Caballo & JY-05-30-B & 4.2 & 450 & 11 & 1,900 & 0.66 & 1.9 & 26 & 14 & 30 \\
\hline Leach 4 & Salt Pan & JY03-25a-B & 6.1 & 210 & 7.5 & 2,900 & 0.61 & 1.4 & 42 & 11 & 55 \\
\hline Leach 4 & Salt Pan & JY03-25b-B & 7.4 & 220 & 9.3 & 3,100 & 0.70 & 1.5 & 49 & 11 & 68 \\
\hline Leach 4 & Salt Pan & JY03-25c-B & 6.6 & 190 & 7.6 & 2,900 & 0.59 & 1.4 & 44 & 10 & 61 \\
\hline Bulk Leach & Salt Pan & JY03-26a & 5.1 & 260 & 6.8 & 2,300 & 0.52 & 1.3 & 35 & 10 & 49 \\
\hline Bulk Leach & Salt Pan & JY03-26b & 7.2 & 240 & 8.6 & 2,800 & 0.63 & 1.6 & 50 & 10 & 57 \\
\hline Bulk Leach & Salt Pan & JY03-26c & 9.6 & 190 & 14 & 3,600 & 0.89 & 2.0 & 64 & 11 & 79 \\
\hline Leach 3 & Salt Pan & JY03-43a-B1 & 7.8 & 270 & 11 & 3,300 & 0.76 & 1.8 & 56 & 12 & 69 \\
\hline Leach 3 & Salt Pan & JY03-43a-B2 & 7.6 & 270 & 11 & 3,200 & 0.75 & 1.9 & 54 & 12 & 68 \\
\hline Leach 4 & Salt Pan & JY03-43b-B1 & 8.6 & 180 & 8.8 & 3,400 & 0.72 & 1.5 & 53 & 11 & 80 \\
\hline Leach 4 & Salt Pan & JY03-43b-B2 & 8.7 & 190 & 9.2 & 3,200 & 0.65 & 1.6 & 54 & 11 & 81 \\
\hline Leach 4 & Salt Pan & JY03-43c-B & 8.4 & 170 & 9.1 & 3,200 & 0.74 & 1.6 & 54 & 10 & 80 \\
\hline
\end{tabular}


Table 12G. Composition of the DI-leach fine fraction of grab samples. Method of determination (ICP-MS, inductively coupled plasma-mass spectrometry; ICP-OES, inductively coupled plasma-optical emission spectrometry; IC, ion chromatograph) is listed above each element.

[Leach batch, denotes the batch in which the sample was processed and analyzed; Sample site, name of sampling site; Sample ID, unique sample identifier; Treatment, type of sample preparation; $-1,-2$, denotes duplicates; n.d., not determined; $\mu \mathrm{g} / \mathrm{L}$, micrograms per liter; $\mathrm{mg} / \mathrm{L}, \mathrm{milligrams} \mathrm{per} \mathrm{liter;} \mathrm{mg} / \mathrm{L} \mathrm{CaCO}{ }_{3}$, milligrams per liter calcium carbonate]

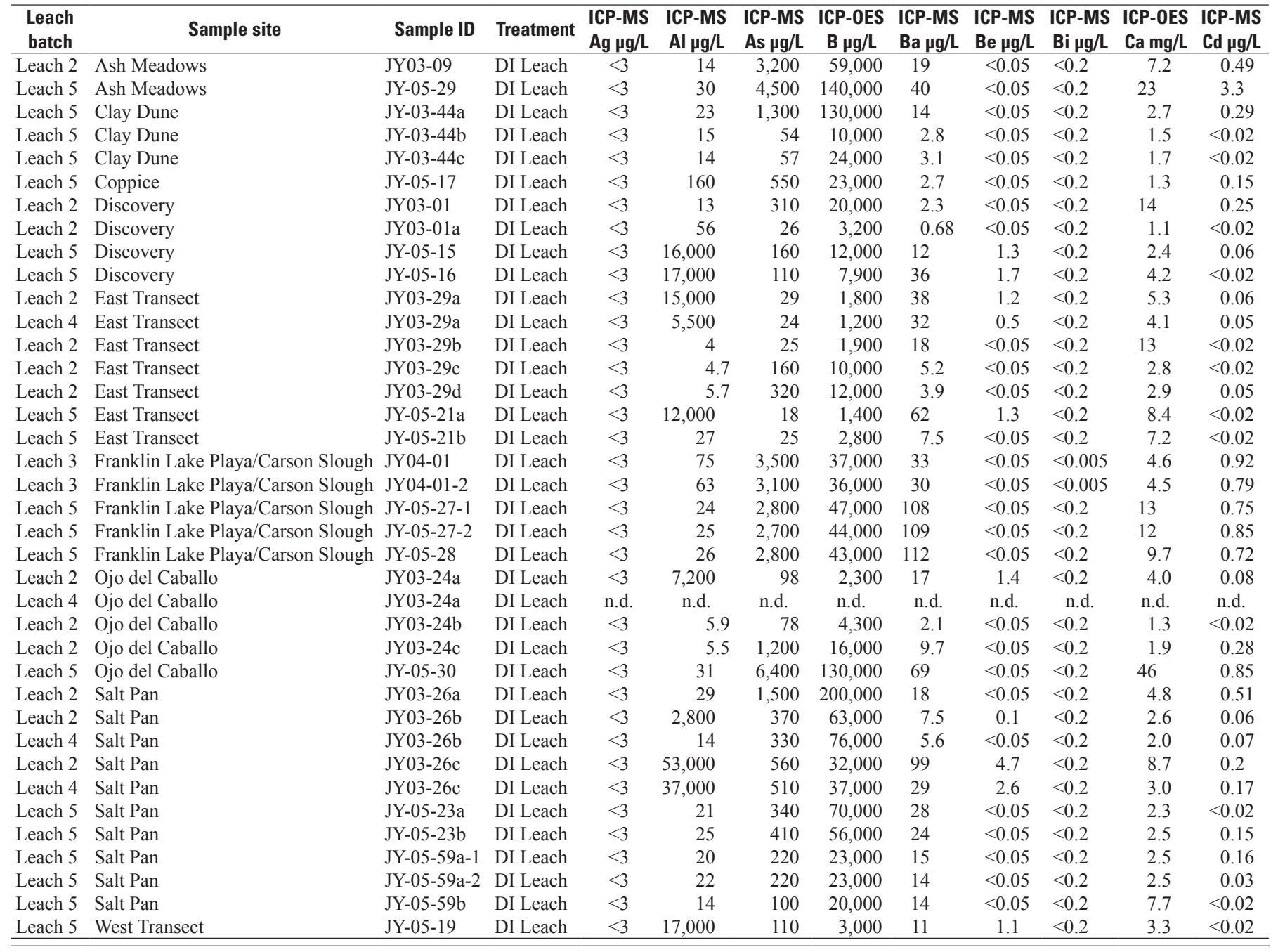


Table 12G. Composition of the DI-leach fine fraction of grab samples. Method of determination (ICP-MS, inductively coupled plasma-mass spectrometry; ICP-OES, inductively coupled plasma-optical emission spectrometry; IC, ion chromatograph) is listed above each element.-Continued

[Leach batch, denotes the batch in which the sample was processed and analyzed; Sample site, name of sampling site; Sample ID, unique sample identifier;

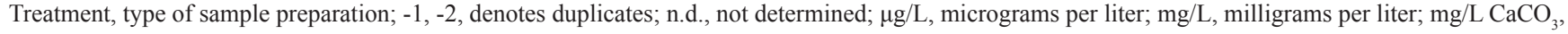
milligrams per liter calcium carbonate]

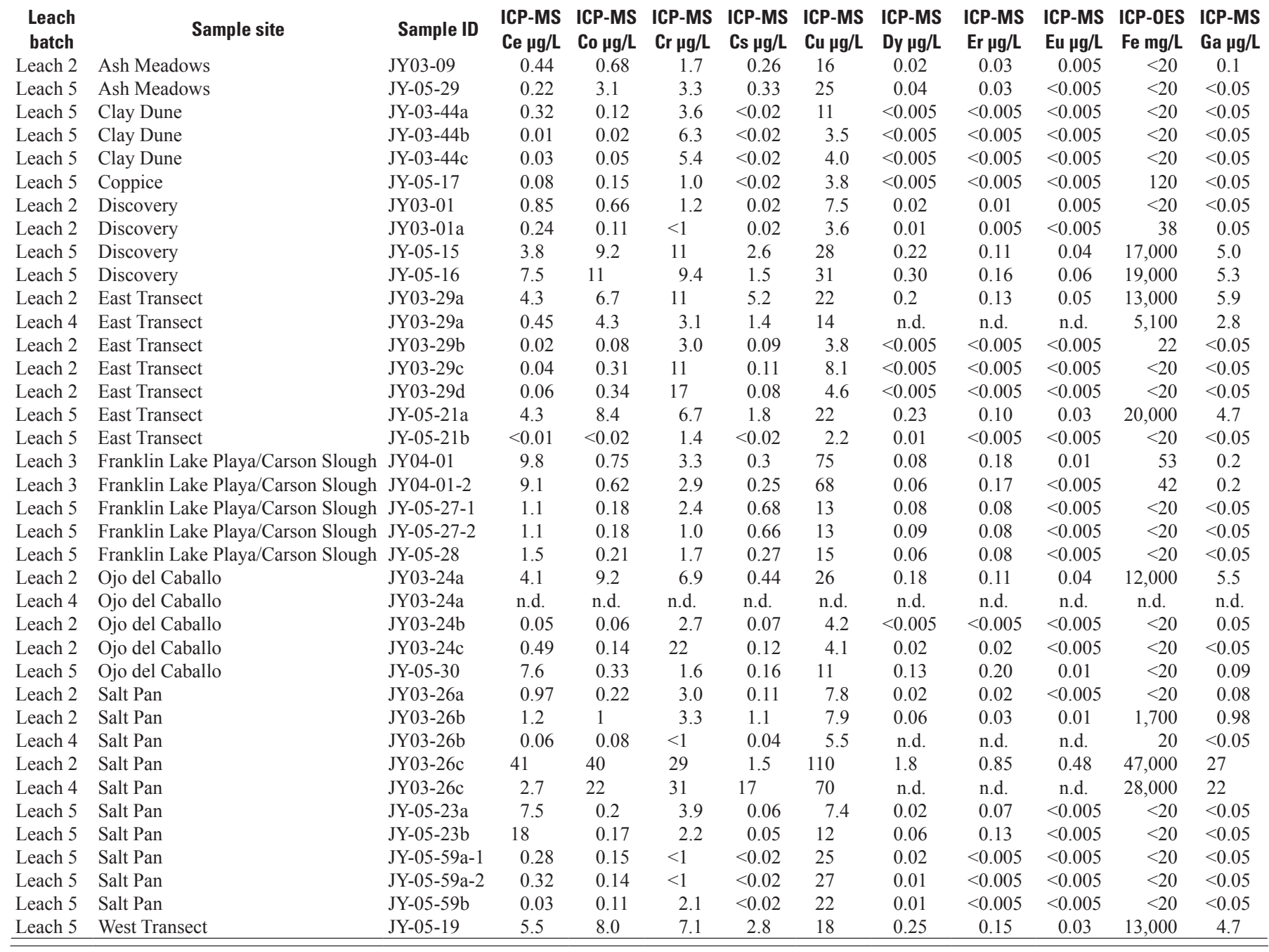


Table 12G. Composition of the DI-leach fine fraction of grab samples. Method of determination (ICP-MS, inductively coupled plasma-mass spectrometry; ICP-OES, inductively coupled plasma-optical emission spectrometry; IC, ion chromatograph) is listed above each element.-Continued

[Leach batch, denotes the batch in which the sample was processed and analyzed; Sample site, name of sampling site; Sample ID, unique sample identifier; Treatment, type of sample preparation; $-1,-2$, denotes duplicates; n.d., not determined; $\mu \mathrm{g} / \mathrm{L}$, micrograms per liter; $\mathrm{mg} / \mathrm{L}, \mathrm{milligrams} \mathrm{per} \mathrm{liter;} \mathrm{mg} / \mathrm{L} \mathrm{CaCO}{ }_{3}$, milligrams per liter calcium carbonate]

\begin{tabular}{|c|c|c|c|c|c|c|c|c|c|c|c|c|}
\hline $\begin{array}{l}\text { Leach } \\
\text { batch }\end{array}$ & Sample site & Sample ID & $\begin{array}{l}\text { ICP-MS } \\
\text { Gd } \mu \mathrm{g} / \mathrm{L}\end{array}$ & $\begin{array}{l}\text { ICP-MS } \\
\text { Ge } \mu \mathrm{g} / \mathrm{L}\end{array}$ & $\begin{array}{l}\text { ICP-MS } \\
\text { Ho } \mu \mathrm{g} / \mathrm{L}\end{array}$ & $\begin{array}{c}\text { ICP-OES } \\
\text { K mg/L }\end{array}$ & $\begin{array}{l}\text { ICP-MS } \\
\text { La } \mu \mathrm{g} / \mathrm{L}\end{array}$ & $\begin{array}{c}\text { ICP-OES } \\
\text { Li } \mu \mathrm{g} / \mathrm{L}\end{array}$ & $\begin{array}{l}\text { ICP-MS } \\
\text { Lu } \mu \mathrm{g} / \mathrm{L}\end{array}$ & $\begin{array}{l}\text { ICP-MS } \\
\text { Mg mg/L }\end{array}$ & $\begin{array}{l}\text { ICP-MS } \\
\mathrm{Mn} \mu \mathrm{g} / \mathrm{L}\end{array}$ & $\begin{array}{l}\text { ICP-MS } \\
\text { Mo } \mu \mathrm{g} / \mathrm{L}\end{array}$ \\
\hline Leach 5 & Ash Meadows & JY-05-29 & 0.01 & 0.1 & 0.006 & 260 & $<0.01$ & 26 & $<0.1$ & 1.3 & 7.3 & 2,800 \\
\hline Leach 5 & Clay Dune & JY-03-44a & 0.02 & 0.07 & $<0.005$ & 33 & $<0.01$ & 370 & $<0.1$ & 0.07 & 0.5 & 290 \\
\hline Leach 5 & Clay Dune & JY-03-44c & 0.007 & 0.06 & $<0.005$ & 15 & $<0.01$ & 16 & $<0.1$ & 0.02 & $<0.2$ & 3.4 \\
\hline Leach 5 & Coppice & JY-05-17 & 0.02 & $<0.05$ & $<0.005$ & 28 & 0.05 & 7.2 & $<0.1$ & 0.20 & 4.8 & 210 \\
\hline Leach 2 & Discovery & JY03-01 & 0.02 & 0.05 & $<0.005$ & 16 & 0.40 & 4.9 & $<0.1$ & 0.02 & 0.3 & 190 \\
\hline Leach 5 & Discovery & JY-05-16 & 0.42 & 0.58 & 0.073 & 22 & 3.0 & 360 & $<0.1$ & 49 & 870 & $<2$ \\
\hline Leach 2 & East Transect & JY03-29a & 0.24 & 1.5 & 0.04 & 15 & 1.7 & 310 & $<0.1$ & 55 & 550 & $<2$ \\
\hline Leach 4 & East Transect & JY03-29a & n.d. & 0.93 & n.d. & 7.1 & 0.13 & 160 & n.d. & 34 & 290 & $<2$ \\
\hline Leach 2 & East Transect & JY03-29b & $<0.005$ & 0.21 & $<0.005$ & 18 & 0.01 & 15 & $<0.1$ & 0.26 & $<0.2$ & $<2$ \\
\hline Leach 2 & East Transect & JY03-29c & $<0.005$ & 0.08 & $<0.005$ & 43 & 0.01 & 7.1 & $<0.1$ & 0.02 & $<0.2$ & 9.3 \\
\hline Leach 2 & East Transect & JY03-29d & $<0.005$ & $<0.05$ & $<0.005$ & 53 & 0.02 & 5.0 & $<0.1$ & 0.01 & $<0.2$ & 53 \\
\hline Leach 5 & Franklin Lake Playa/Carson Slough & JY-05-27-2 & 0.04 & $<0.05$ & 0.02 & 550 & 0.03 & 2,300 & $<0.1$ & 0.01 & 0.2 & 650 \\
\hline Leach 5 & Franklin Lake Playa/Carson Slough & JY-05-28 & 0.04 & 0.3 & 0.02 & 310 & 0.03 & 1,300 & $<0.1$ & 0.02 & 0.6 & 670 \\
\hline Leach 2 & Ojo del Caballo & JY03-24a & 0.24 & 1.3 & 0.03 & 16 & 1.4 & 340 & $<0.1$ & 74 & 690 & 6.3 \\
\hline Leach 4 & Ojo del Caballo & JY03-24a & n.d. & n.d. & n.d. & n.d. & n.d. & n.d. & n.d. & n.d. & n.d. & n.d. \\
\hline Leach 2 & Ojo del Caballo & JY03-24b & $<0.005$ & 0.1 & $<0.005$ & 15 & 0.02 & 6.7 & $<0.1$ & 0.01 & $<0.2$ & 3.8 \\
\hline Leach 2 & Ojo del Caballo & JY03-24c & 0.02 & 0.09 & 0.006 & 36 & 0.07 & 5.3 & $<0.1$ & 0.01 & $<0.2$ & 240 \\
\hline Leach 5 & Ojo del Caballo & JY-05-30 & 0.083 & $<0.05$ & 0.054 & 180 & 0.12 & 3.4 & $<0.1$ & 0.20 & 2.1 & 760 \\
\hline Leach 2 & Salt Pan & JY03-26a & 0.009 & 0.2 & 0.006 & 22 & 0.03 & 190 & $<0.1$ & 0.14 & 0.5 & 410 \\
\hline Leach 2 & Salt Pan & JY03-26b & 0.062 & 0.38 & 0.01 & 18 & 0.50 & 250 & $<0.1$ & 4.8 & 74 & 53 \\
\hline Leach 4 & Salt Pan & JY03-26b & n.d. & 0.1 & n.d. & 16 & 0.02 & 200 & n.d. & 0.02 & 0.3 & 45 \\
\hline Leach 2 & Salt Pan & JY03-26c & 2.46 & 4 & 0.32 & 67 & 15 & 6,400 & 0.1 & 310 & 3,300 & 11 \\
\hline Leach 4 & Salt Pan & JY03-26c & n.d. & 9.2 & n.d. & 43 & 0.30 & 2,900 & n.d. & 170 & 1,600 & 74 \\
\hline Leach 5 & Salt Pan & JY-05-23a & 0.01 & $<0.05$ & 0.01 & 22 & $<0.01$ & 21 & $<0.1$ & 0.31 & 0.4 & 53 \\
\hline
\end{tabular}


Table 12G. Composition of the DI-leach fine fraction of grab samples. Method of determination (ICP-MS, inductively coupled plasma-mass spectrometry; ICP-OES, inductively coupled plasma-optical emission spectrometry; IC, ion chromatograph) is listed above each element.-Continued

[Leach batch, denotes the batch in which the sample was processed and analyzed; Sample site, name of sampling site; Sample ID, unique sample identifier; Treatment, type of sample preparation; -1, -2, denotes duplicates; n.d., not determined; $\mu \mathrm{g} / \mathrm{L}$, micrograms per liter; $\mathrm{mg} / \mathrm{L}, \mathrm{milligrams} \mathrm{per} \mathrm{liter;} \mathrm{mg} / \mathrm{L} \mathrm{CaCO}$, milligrams per liter calcium carbonate]

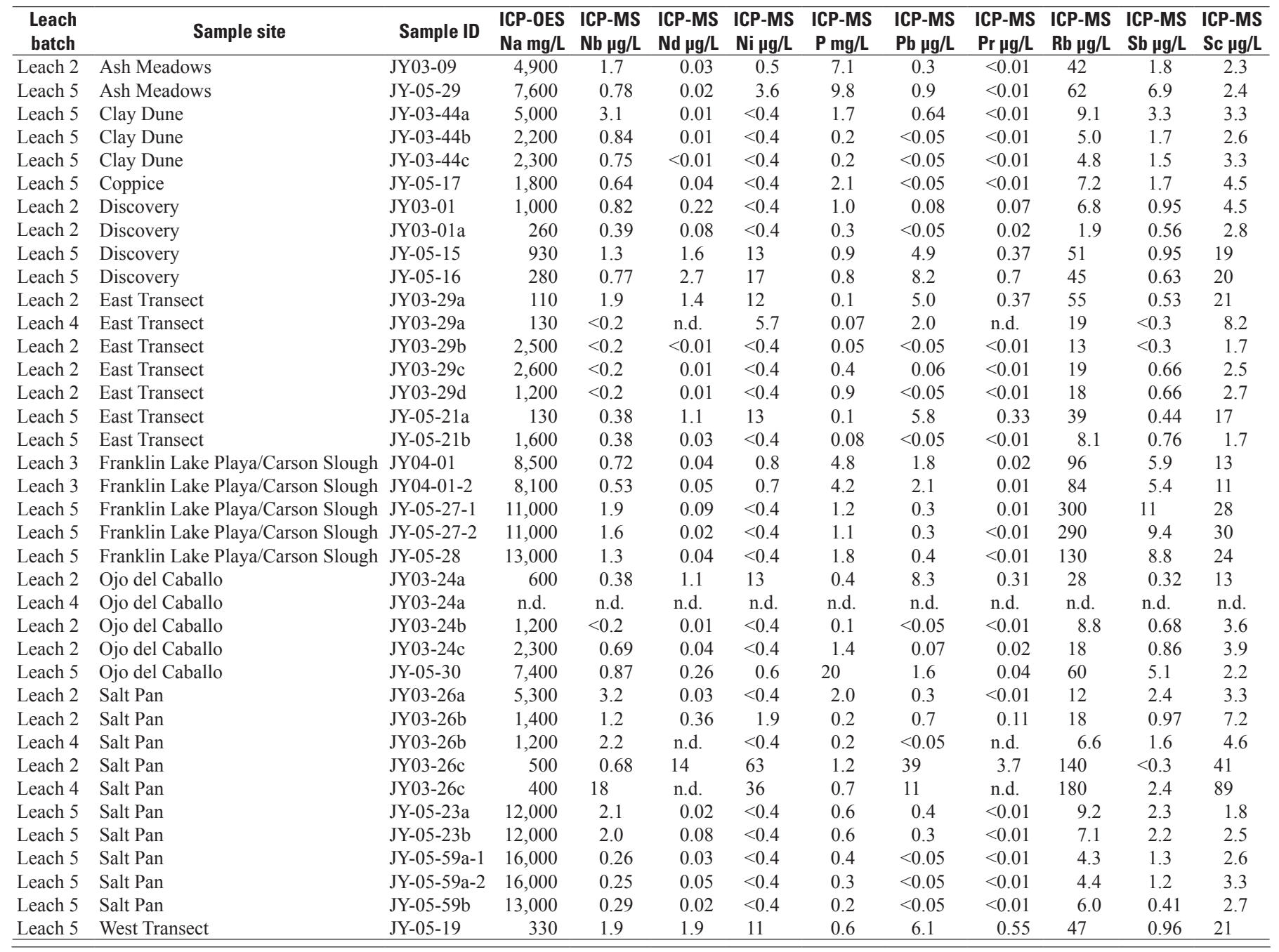


Table 12G. Composition of the DI-leach fine fraction of grab samples. Method of determination (ICP-MS, inductively coupled plasma-mass spectrometry; ICP-OES, inductively coupled plasma-optical emission spectrometry; IC, ion chromatograph) is listed above each element.-Continued

[Leach batch, denotes the batch in which the sample was processed and analyzed; Sample site, name of sampling site; Sample ID, unique sample identifier; Treatment, type of sample preparation; $-1,-2$, denotes duplicates; n.d., not determined; $\mu \mathrm{g} / \mathrm{L}$, micrograms per liter; $\mathrm{mg} / \mathrm{L}, \mathrm{milligrams} \mathrm{per}$ liter; $\mathrm{mg} / \mathrm{L} \mathrm{CaCO}{ }_{3}$, milligrams per liter calcium carbonate]

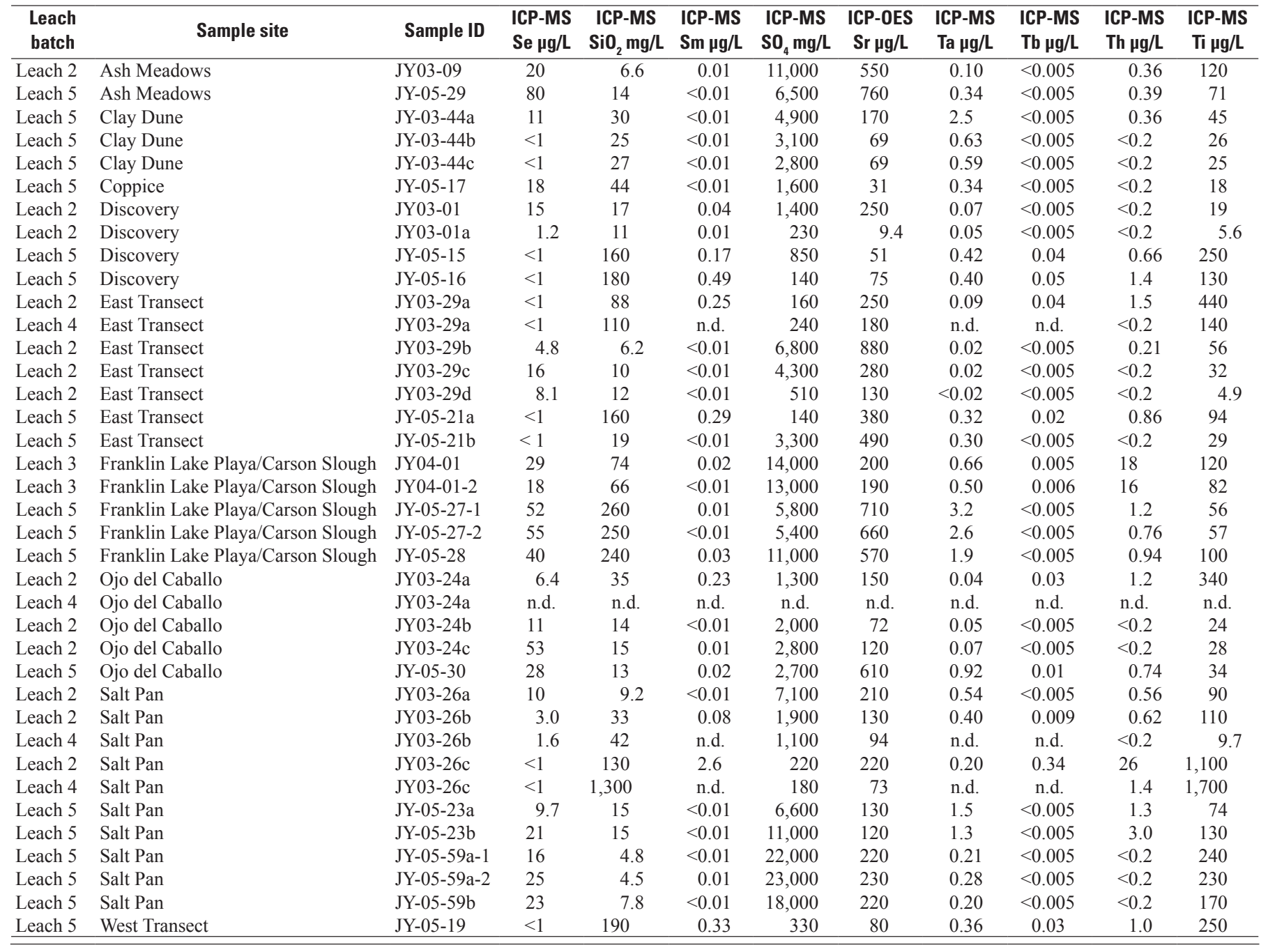


Table 12G. Composition of the DI-leach fine fraction of grab samples. Method of determination (ICP-MS, inductively coupled plasma-mass spectrometry; ICP-OES, inductively coupled plasma-optical emission spectrometry; IC, ion chromatograph) is listed above each element.-Continued

[Leach batch, denotes the batch in which the sample was processed and analyzed; Sample site, name of sampling site; Sample ID, unique sample identifier; Treatment, type of sample preparation; $-1,-2$, denotes duplicates; n.d., not determined; $\mu \mathrm{g} / \mathrm{L}$, micrograms per liter; $\mathrm{mg} / \mathrm{L}, \mathrm{milligrams} \mathrm{per} \mathrm{liter;} \mathrm{mg} / \mathrm{L} \mathrm{CaCO}{ }_{3}$, milligrams per liter calcium carbonate]

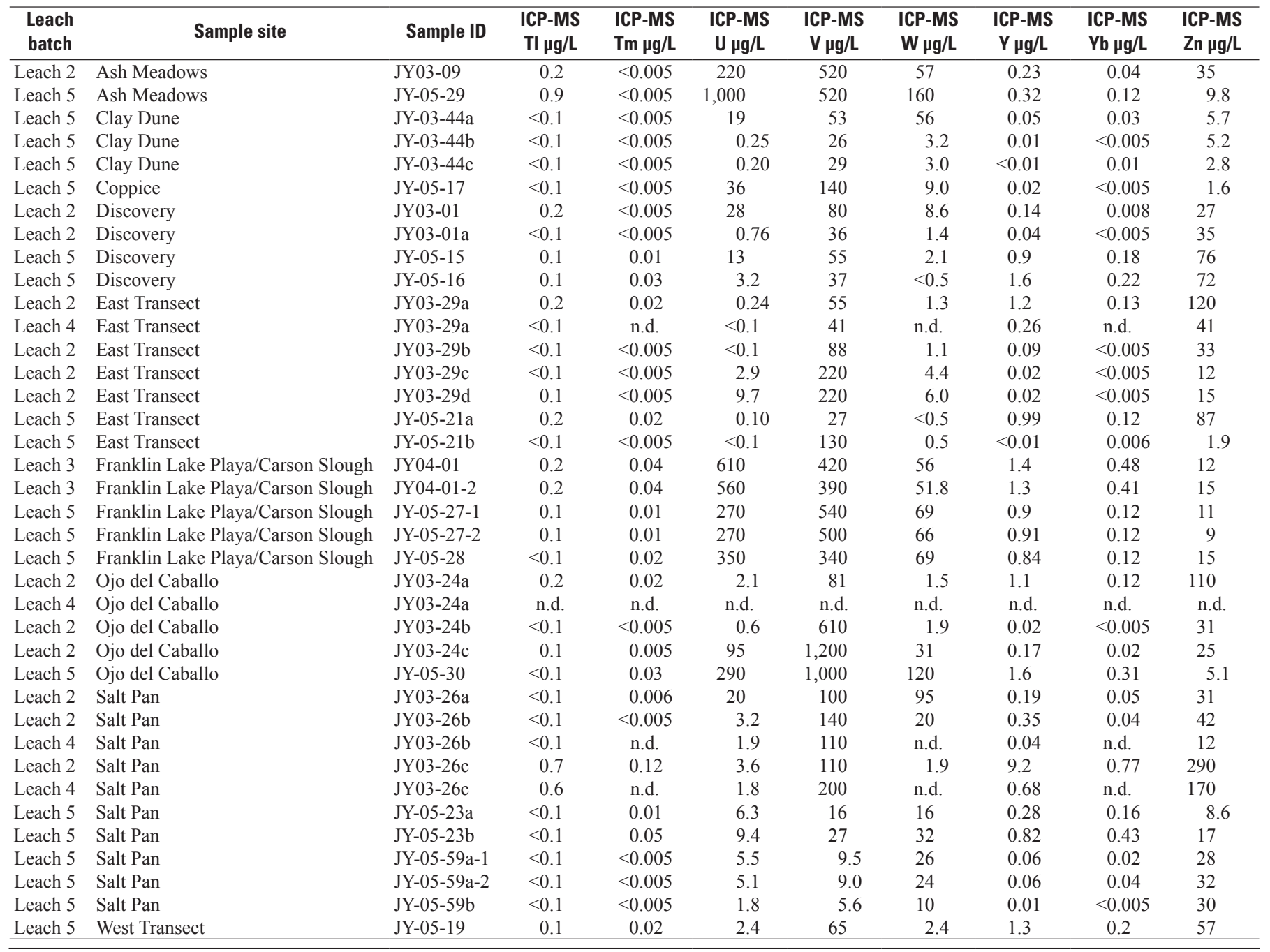


Table 12G. Composition of the DI-leach fine fraction of grab samples. Method of determination (ICP-MS, inductively coupled plasma-mass spectrometry; ICP-OES, inductively coupled plasma-optical emission spectrometry; IC, ion chromatograph) is listed above each element.-Continued

[Leach batch, denotes the batch in which the sample was processed and analyzed; Sample site, name of sampling site; Sample ID, unique sample identifier; Treatment, type of sample preparation; $-1,-2$, denotes duplicates; n.d., not determined; $\mu \mathrm{g} / \mathrm{L}$, micrograms per liter; $\mathrm{mg} / \mathrm{L}, \mathrm{milligrams} \mathrm{per} \mathrm{liter;} \mathrm{mg} / \mathrm{L} \mathrm{CaCO}{ }_{3}$, milligrams per liter calcium carbonate]

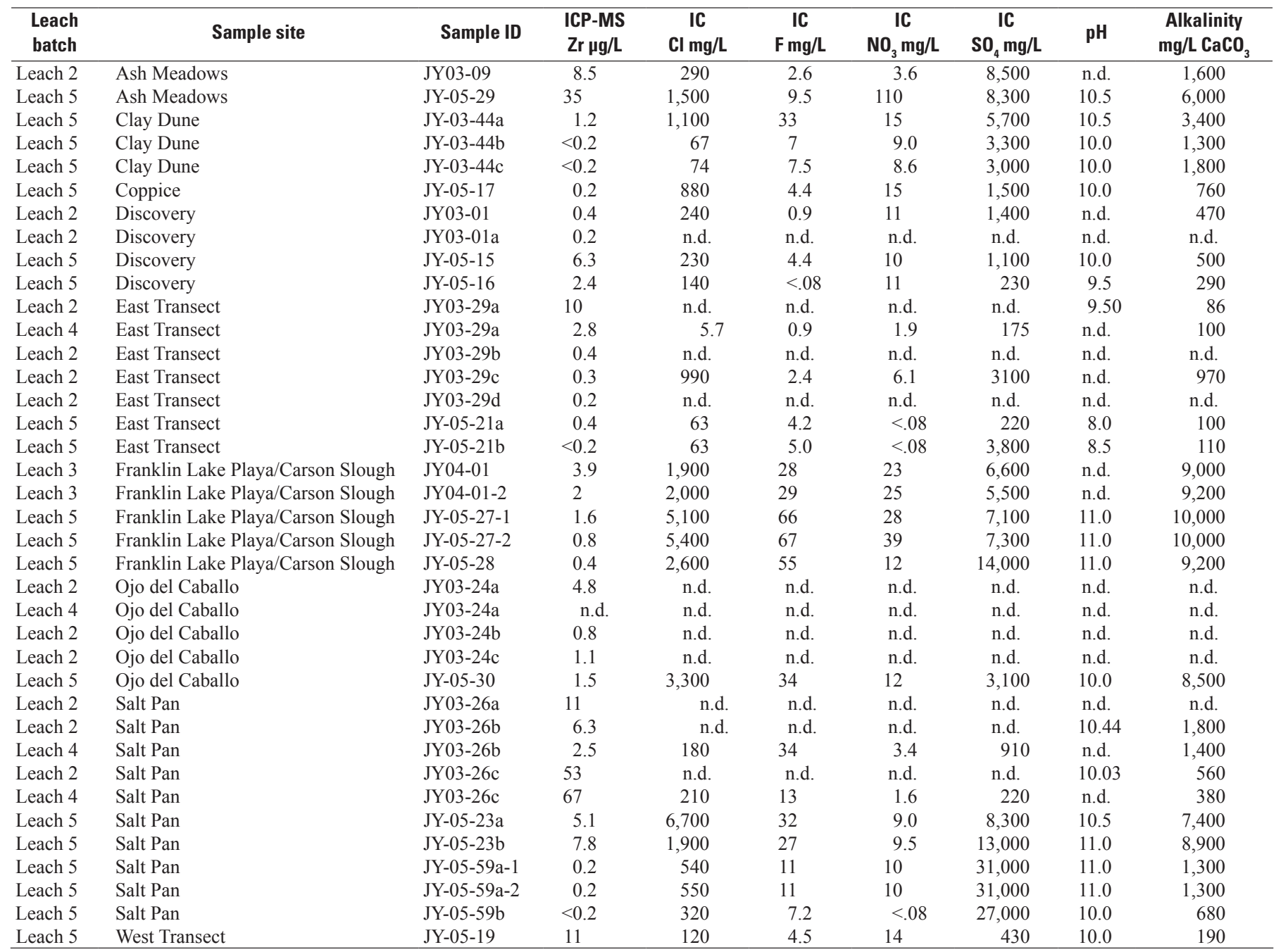


Table 12H. Composition of the nitric acid leach fine fraction of grab samples. Method of determination (ICP-MS, inductively coupled plasma-mass spectrometry; ICP-OES, inductively coupled plasma-optical emission spectrometry) is listed above each element.

[Leach batch, denotes the batch in which the sample was processed and analyzed; Sample site, name of sampling site; Sample ID, unique sample identifier; Treatment, type of sample preparation; -1, -2, denotes duplicates; ins, insufficient sample; $\mu \mathrm{g} / \mathrm{L}$, micrograms per liter; mg/L, milligrams per liter]

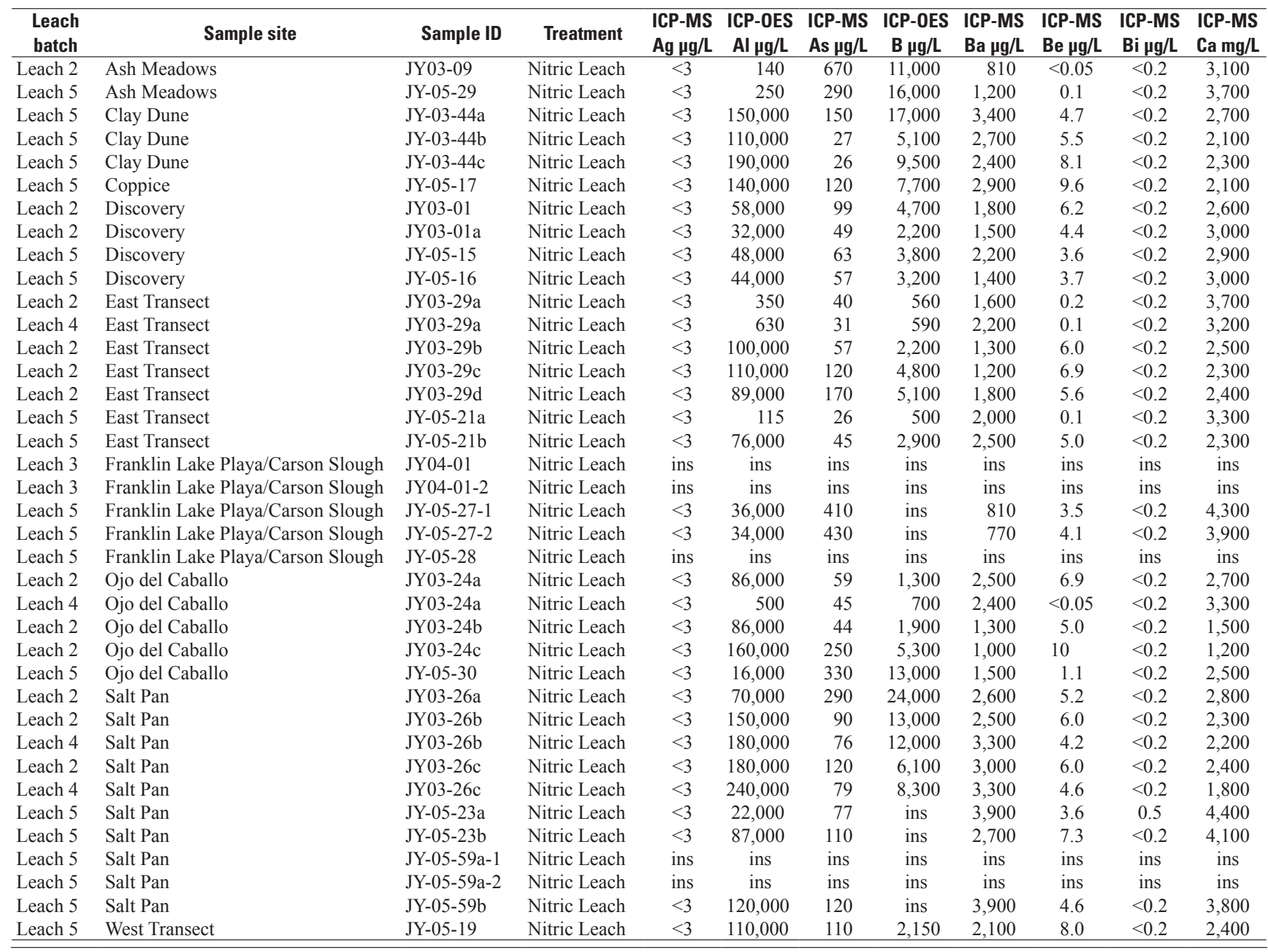


Table 12H. Composition of the nitric acid leach fine fraction of grab samples. Method of determination (ICP-MS, inductively coupled plasma-mass spectrometry; ICP-OES, inductively coupled plasma-optical emission spectrometry) is listed above each element.-Continued

[Leach batch, denotes the batch in which the sample was processed and analyzed; Sample site, name of sampling site; Sample ID, unique sample identifier; Treatment, type of sample preparation; -1, -2, denotes duplicates; ins, insufficient sample; $\mu \mathrm{g} / \mathrm{L}$, micrograms per liter; $\mathrm{mg} / \mathrm{L}$, milligrams per liter]

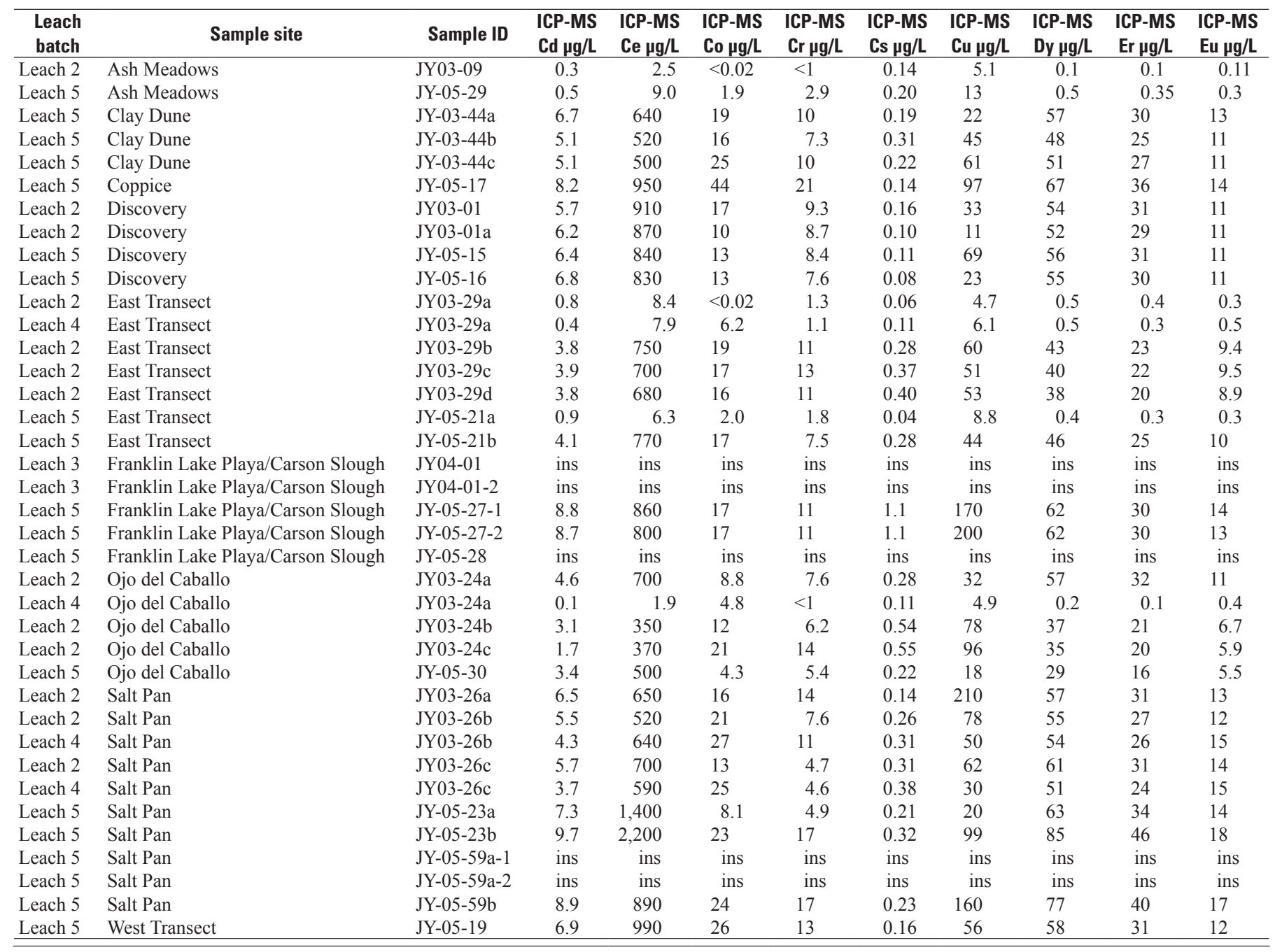


Table 12H. Composition of the nitric acid leach fine fraction of grab samples. Method of determination (ICP-MS, inductively coupled plasma-mass spectrometry; ICP-OES, inductively coupled plasma-optical emission spectrometry) is listed above each element.-Continued

[Leach batch, denotes the batch in which the sample was processed and analyzed; Sample site, name of sampling site; Sample ID, unique sample identifier; Treatment, type of sample preparation; $-1,-2$, denotes duplicates; ins, insufficient sample; $\mu \mathrm{g} / \mathrm{L}$, micrograms per liter; $\mathrm{mg} / \mathrm{L}$, milligrams per liter]

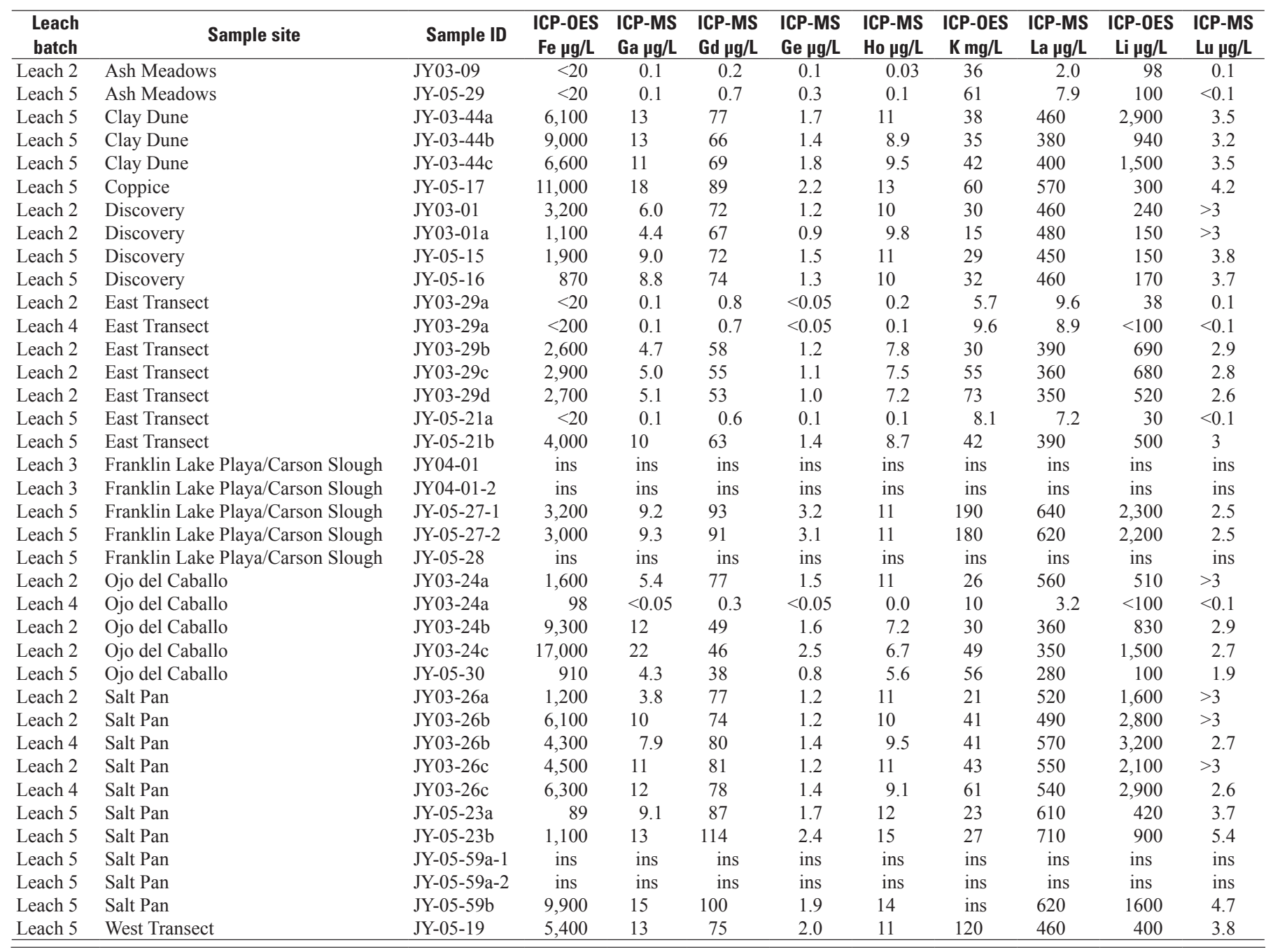


Table 12H. Composition of the nitric acid leach fine fraction of grab samples. Method of determination (ICP-MS, inductively coupled plasma-mass spectrometry; ICP-OES, inductively coupled plasma-optical emission spectrometry) is listed above each element.-Continued

[Leach batch, denotes the batch in which the sample was processed and analyzed; Sample site, name of sampling site; Sample ID, unique sample identifier; Treatment, type of sample preparation; -1, -2, denotes duplicates; ins, insufficient sample; $\mu \mathrm{g} / \mathrm{L}$, micrograms per liter; $\mathrm{mg} / \mathrm{L}$, milligrams per liter]

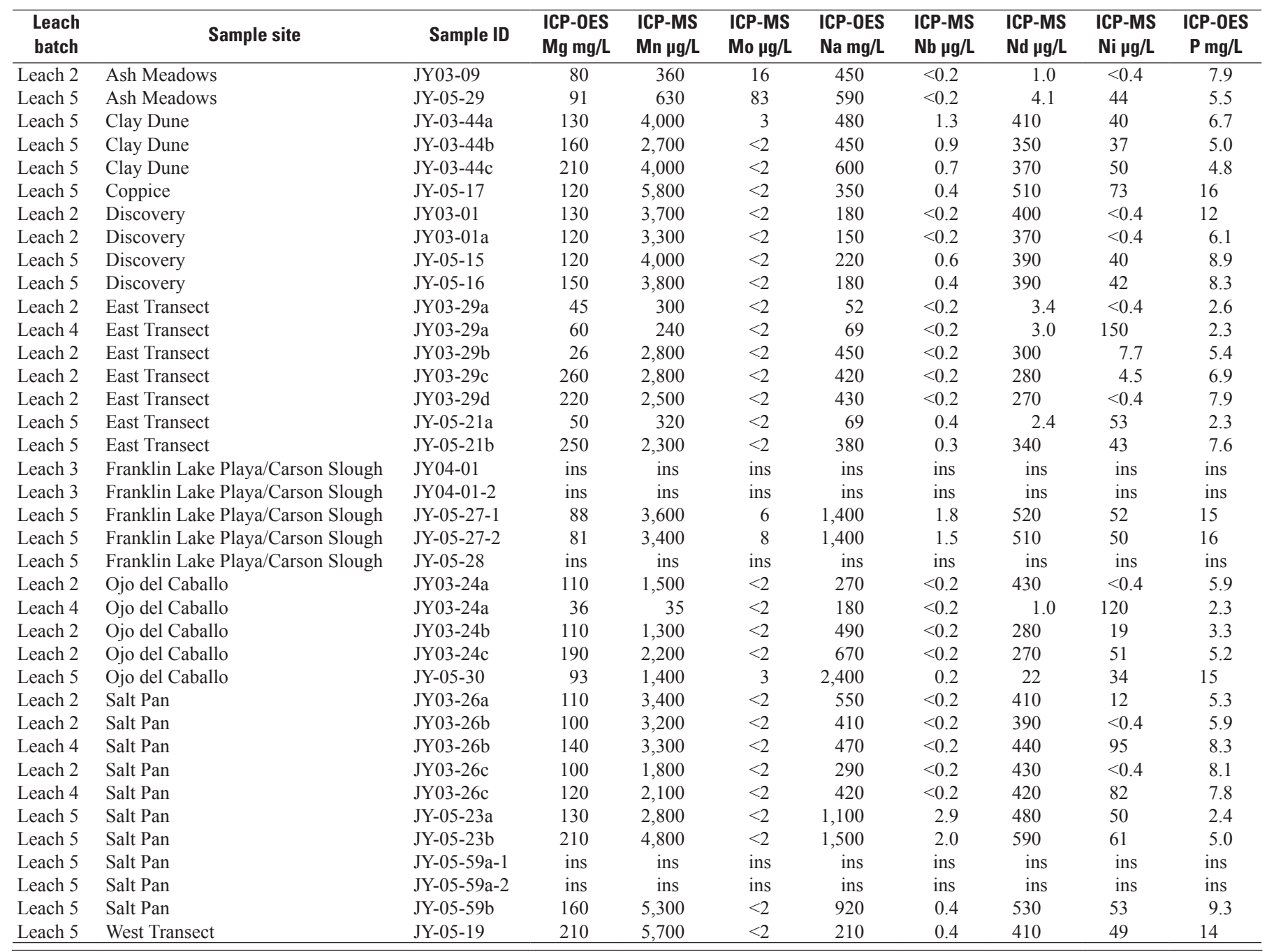


Table 12H. Composition of the nitric acid leach fine fraction of grab samples. Method of determination (ICP-MS, inductively coupled plasma-mass spectrometry; ICP-OES, inductively coupled plasma-optical emission spectrometry) is listed above each element.-Continued

[Leach batch, denotes the batch in which the sample was processed and analyzed; Sample site, name of sampling site; Sample ID, unique sample identifier; Treatment, type of sample preparation; $-1,-2$, denotes duplicates; ins, insufficient sample; $\mu \mathrm{g} / \mathrm{L}$, micrograms per liter; $\mathrm{mg} / \mathrm{L}$, milligrams per liter]

\begin{tabular}{|c|c|c|c|c|c|c|c|c|c|c|}
\hline $\begin{array}{l}\text { Leach } \\
\text { batch }\end{array}$ & Sample site & Sample ID & $\begin{array}{l}\text { ICP-MS } \\
\mathrm{Pb} \mu \mathrm{g} / \mathrm{L}\end{array}$ & $\begin{array}{l}\text { ICP-MS } \\
\text { Pr } \mu g / L\end{array}$ & $\begin{array}{l}\text { ICP-MS } \\
\mathrm{Rb} \mu \mathrm{g} / \mathrm{L}\end{array}$ & $\begin{array}{l}\text { ICP-MS } \\
\text { Sb } \mu g / L\end{array}$ & $\begin{array}{l}\text { ICP-MS } \\
\text { Sc } \mu \mathrm{g} / \mathrm{L}\end{array}$ & $\begin{array}{l}\text { ICP-MS } \\
\text { Se } \mu \mathrm{g} / \mathrm{L}\end{array}$ & $\begin{array}{l}\text { ICP-OES } \\
\mathrm{SiO}_{2} \mathrm{mg} / \mathrm{L}\end{array}$ & $\begin{array}{l}\text { ICP-MS } \\
\text { Sm } \mu g / L\end{array}$ \\
\hline Leach 5 & Ash Meadows & JY-05-29 & 0.4 & 1 & 26 & 5.2 & 7.6 & 6.7 & 48 & 0.6 \\
\hline Leach 5 & Clay Dune & JY-03-44b & 160 & 90 & 30 & 0.7 & 21 & 4.6 & 67 & 67 \\
\hline Leach 5 & Clay Dune & JY-03-44c & 130 & 93 & 28 & 0.8 & 25 & 3.9 & 98 & 72 \\
\hline Leach 5 & Coppice & JY-05-17 & 120 & 130 & 37 & 0.5 & 19 & 4.8 & 90 & 94 \\
\hline Leach 2 & Discovery & JY03-01 & 84 & 110 & 26 & $<0.3$ & 8.7 & 2.6 & 35 & 74 \\
\hline Leach 5 & Discovery & JY-05-16 & 35 & 100 & 18 & 0.5 & 6.6 & 3.8 & 33 & 72 \\
\hline Leach 2 & East Transect & JY03-29a & 0.8 & 0.9 & 5.5 & 0.4 & 4.8 & 3.3 & 12 & 0.6 \\
\hline Leach 4 & East Transect & JY03-29a & 0.6 & 0.8 & 11 & $<0.3$ & 31 & 1.4 & 24 & 0.5 \\
\hline Leach 2 & East Transect & JY03-29b & 91 & 84 & 37 & $<0.3$ & 16 & 2.2 & 54 & 58 \\
\hline Leach 2 & East Transect & JY03-29c & 97 & 79 & 47 & $<0.3$ & 18 & 3.3 & 59 & 55 \\
\hline Leach 2 & East Transect & JY03-29d & 95 & 75 & 55 & $<0.3$ & 15 & 3.0 & 47 & 54 \\
\hline Leach 5 & Franklin Lake Playa/Carson Slough & JY-05-27-1 & 88 & 130 & 260 & 3.3 & 10 & 17 & 85 & 94 \\
\hline Leach 5 & Franklin Lake Playa/Carson Slough & JY-05-27-2 & 88 & 130 & 250 & 3.3 & 10 & 17 & 86 & 91 \\
\hline Leach 5 & Franklin Lake Playa/Carson Slough & JY-05-28 & ins & ins & ins & ins & ins & ins & ins & ins \\
\hline Leach 2 & Ojo del Caballo & JY03-24a & 100 & 120 & 28 & $<0.3$ & 7.5 & 3.4 & 29 & 79 \\
\hline Leach 4 & Ojo del Caballo & JY03-24a & 0.3 & 0.3 & 11 & $<0.3$ & 39 & 1.6 & 33 & 0.2 \\
\hline Leach 2 & Ojo del Caballo & JY03-24b & 120 & 80 & 39 & $<0.3$ & 11 & 2.5 & 37 & 52 \\
\hline Leach 2 & Ojo del Caballo & JY03-24c & 120 & 75 & 52 & $<0.3$ & 17 & 1.2 & 69 & 50 \\
\hline Leach 5 & Ojo del Caballo & JY-05-30 & 25 & 57 & 28 & 1.6 & 5.9 & 4.5 & 45 & 37 \\
\hline Leach 2 & Salt Pan & JY03-26a & 110 & 110 & 20 & 0.5 & 11 & 3.4 & 48 & 78 \\
\hline Leach 2 & Salt Pan & JY03-26b & 140 & 110 & 35 & $<0.3$ & 16 & 1.9 & 62 & 76 \\
\hline Leach 4 & Salt Pan & JY03-26b & 150 & 120 & 38 & $<0.3$ & 87 & $<1$ & 86 & 86 \\
\hline Leach 2 & Salt Pan & JY03-26c & 61 & 120 & 36 & $<0.3$ & 14 & 2.3 & 50 & 83 \\
\hline Leach 4 & Salt Pan & JY03-26c & 92 & 120 & 43 & $<0.3$ & 100 & $<1$ & 90 & 82 \\
\hline
\end{tabular}


Table 12H. Composition of the nitric acid leach fine fraction of grab samples. Method of determination (ICP-MS, inductively coupled plasma-mass spectrometry; ICP-OES, inductively coupled plasma-optical emission spectrometry) is listed above each element.-Continued

[Leach batch, denotes the batch in which the sample was processed and analyzed; Sample site, name of sampling site; Sample ID, unique sample identifier; Treatment, type of sample preparation; -1, -2, denotes duplicates; ins, insufficient sample; $\mu \mathrm{g} / \mathrm{L}$, micrograms per liter; $\mathrm{mg} / \mathrm{L}$, milligrams per liter]

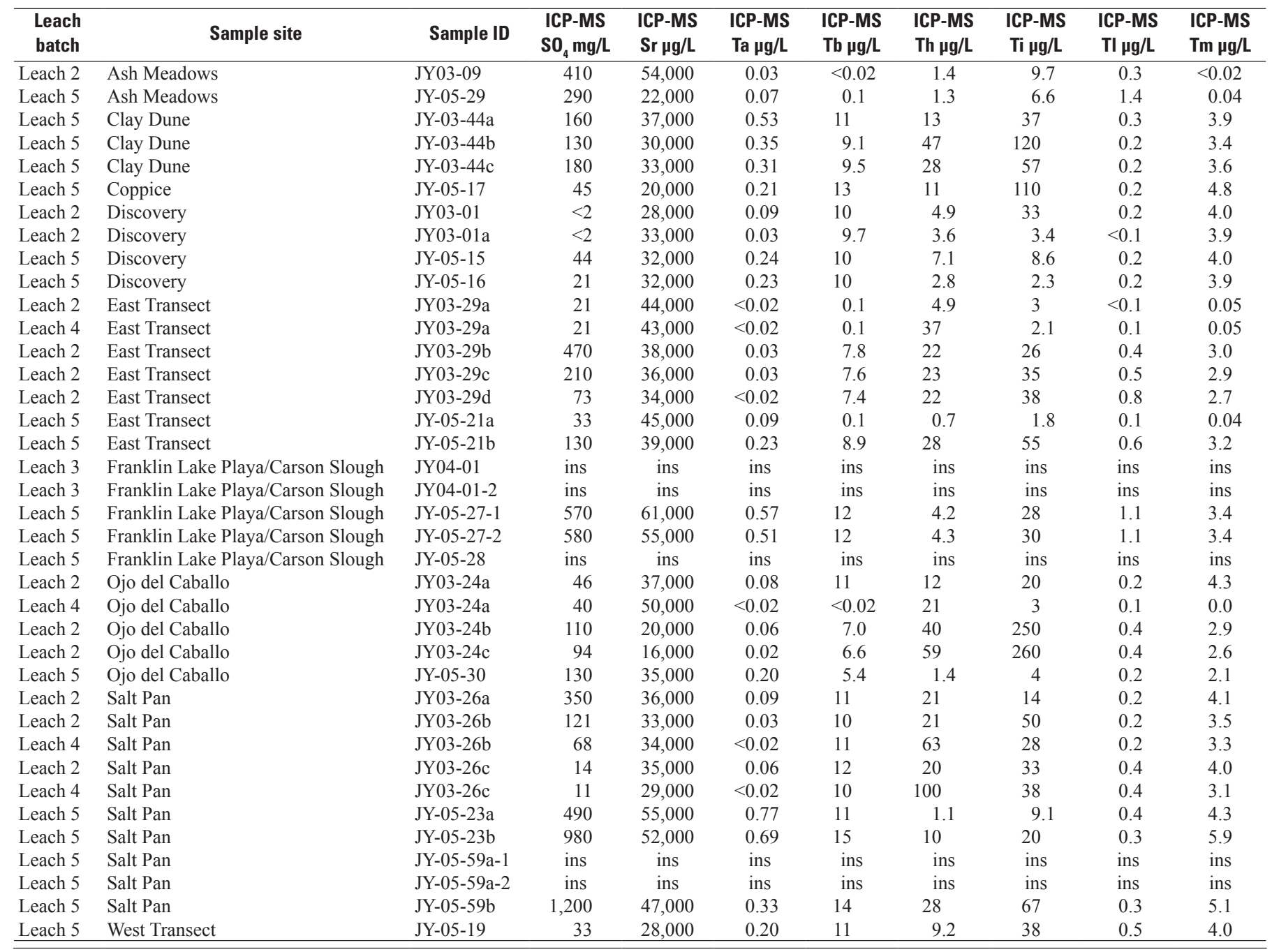


Table 12H. Composition of the nitric acid leach fine fraction of grab samples. Method of determination (ICP-MS, inductively coupled plasma-mass spectrometry; ICP-OES, inductively coupled plasma-optical emission spectrometry) is listed above each element.-Continued

[Leach batch, denotes the batch in which the sample was processed and analyzed; Sample site, name of sampling site; Sample ID, unique sample identifier; Treatment, type of sample preparation; -1, -2, denotes duplicates; ins, insufficient sample; $\mu \mathrm{g} / \mathrm{L}$, micrograms per liter; $\mathrm{mg} / \mathrm{L}$, milligrams per liter]

\begin{tabular}{|c|c|c|c|c|c|c|c|c|c|}
\hline $\begin{array}{l}\text { Leach } \\
\text { batch }\end{array}$ & Sample site & Sample ID & $\begin{array}{c}\text { ICP-MS } \\
\text { U } \mu \mathrm{g} / \mathrm{L}\end{array}$ & $\begin{array}{c}\text { ICP-MS } \\
\text { V } \mu \mathrm{g} / \mathrm{L}\end{array}$ & $\begin{array}{l}\text { ICP-MS } \\
W \mu g / L\end{array}$ & $\begin{array}{c}\text { ICP-MS } \\
Y \mu g / L\end{array}$ & $\begin{array}{l}\text { ICP-MS } \\
\mathrm{Yb} \mu \mathrm{g} / \mathrm{L}\end{array}$ & $\begin{array}{l}\text { ICP-MS } \\
\mathrm{Zn} \mu \mathrm{g} / \mathrm{L}\end{array}$ & $\begin{array}{l}\text { ICP-MS } \\
\mathrm{Zr} \mu \mathrm{g} / \mathrm{L}\end{array}$ \\
\hline Leach 5 & Ash Meadows & JY-05-29 & 24 & 10 & 5.3 & 4.3 & 0.3 & 8.2 & 0.9 \\
\hline Leach 5 & Clay Dune & $J Y-03-44 b$ & 3.0 & 7.2 & 0.9 & 250 & 22 & 180 & 1.2 \\
\hline Leach 5 & Clay Dune & $J Y-03-44 c$ & 3.1 & 3.3 & 0.9 & 270 & 25 & 120 & 1.4 \\
\hline Leach 5 & Coppice & JY-05-17 & 19 & 37 & 0.7 & 350 & 31 & 340 & 5.5 \\
\hline Leach 2 & Discovery & JY03-01 & 11 & 19 & 1.1 & 290 & 26 & 110 & 4.8 \\
\hline Leach 5 & Discovery & JY-05-16 & 4.3 & 2 & 0.7 & 280 & 26 & 57 & 1.8 \\
\hline Leach 2 & East Transect & JY03-29a & 3.0 & 1.6 & 0.5 & 6.8 & 0.3 & 4.2 & 0.7 \\
\hline Leach 4 & East Transect & JY03-29a & 4.6 & 1.4 & $<0.5$ & 5.8 & 0.3 & 5.4 & 1.3 \\
\hline Leach 2 & East Transect & JY03-29b & 6.9 & 7.0 & 0.6 & 230 & 19 & 140 & 1.4 \\
\hline Leach 2 & East Transect & JY03-29c & 12 & 10 & 0.6 & 210 & 19 & 120 & 1.9 \\
\hline Leach 2 & East Transect & JY03-29d & 22 & 13 & $<0.5$ & 210 & 18 & 100 & 3.0 \\
\hline Leach 5 & Franklin Lake Playa/Carson Slough & $J Y-05-27-1$ & 23 & 150 & 6.5 & 300 & 20 & 2,800 & 2.3 \\
\hline Leach 5 & Franklin Lake Playa/Carson Slough & $J Y-05-27-2$ & 24 & 150 & 6.4 & 290 & 20 & 3,300 & 2.5 \\
\hline Leach 5 & Franklin Lake Playa/Carson Slough & JY-05-28 & ins & ins & ins & ins & ins & ins & ins \\
\hline Leach 2 & Ojo del Caballo & JY03-24a & 6.1 & 3.1 & 0.9 & 320 & 27 & 120 & 1.3 \\
\hline Leach 4 & Ojo del Caballo & JY03-24a & 6.0 & 0.8 & $<0.5$ & 2.3 & 0.08 & 11 & 1.2 \\
\hline Leach 2 & Ojo del Caballo & JY03-24b & 3.3 & 32 & 0.7 & 210 & 19 & 170 & 1.1 \\
\hline Leach 2 & Ojo del Caballo & JY03-24c & 24 & 57 & 0.6 & 200 & 17 & 300 & 1.5 \\
\hline Leach 5 & Ojo del Caballo & JY-05-30 & 1.5 & 10 & 1.1 & 170 & 13 & 240 & 1.8 \\
\hline Leach 2 & Salt Pan & JY03-26a & 3.9 & 5.9 & 1.4 & 310 & 24 & 340 & 2.6 \\
\hline Leach 2 & Salt Pan & JY03-26b & 3.9 & 9.1 & 0.7 & 280 & 22 & 150 & 1.8 \\
\hline Leach 4 & Salt Pan & JY03-26b & 5.9 & 4.9 & $<0.5$ & 260 & 21 & 97 & 1.3 \\
\hline Leach 2 & Salt Pan & JY03-26c & 4.9 & 5.7 & 0.5 & 320 & 26 & 96 & 1.1 \\
\hline Leach 4 & Salt Pan & JY03-26c & 9.5 & 4.6 & $<0.5$ & 250 & 20 & 83 & 1.2 \\
\hline
\end{tabular}


Table 12I. Composition of the residual fraction of fine fraction of grab samples. Method of determination (ICP-MS, inductively coupled plasma-mass spectrometry; ICP-OES, inductively coupled plasma-optical emission spectrometry) is listed above each element.

[Leach batch, denotes the batch in which the sample was processed and analyzed; Sample site, name of sampling site; Sample ID, unique sample identifier; Treatment, type of sample preparation; $-1,-2$, denotes duplicates; ins, insufficient sample; ppm, parts per million; wt.\%, weight percent]

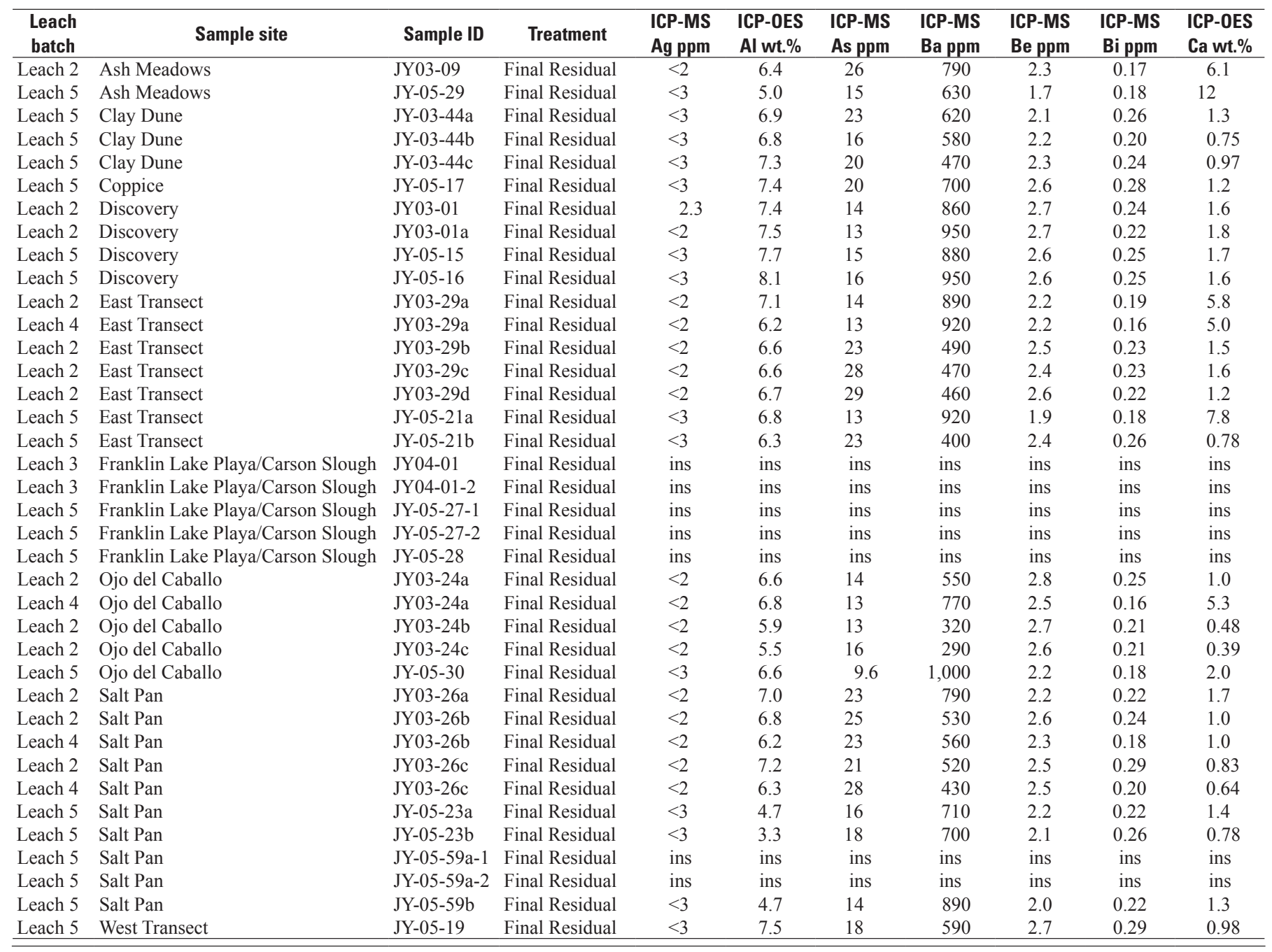


Table 12I. Composition of the residual fraction of fine fraction of grab samples. Method of determination (ICP-MS, inductively coupled plasma-mass spectrometry; ICP-OES, inductively coupled plasma-optical emission spectrometry) is listed above each element.-Continued

[Leach batch, denotes the batch in which the sample was processed and analyzed; Sample site, name of sampling site; Sample ID, unique sample identifier; Treatment, type of sample preparation; -1, -2, denotes duplicates; ins, insufficient sample; ppm, parts per million; wt.\%, weight percent]

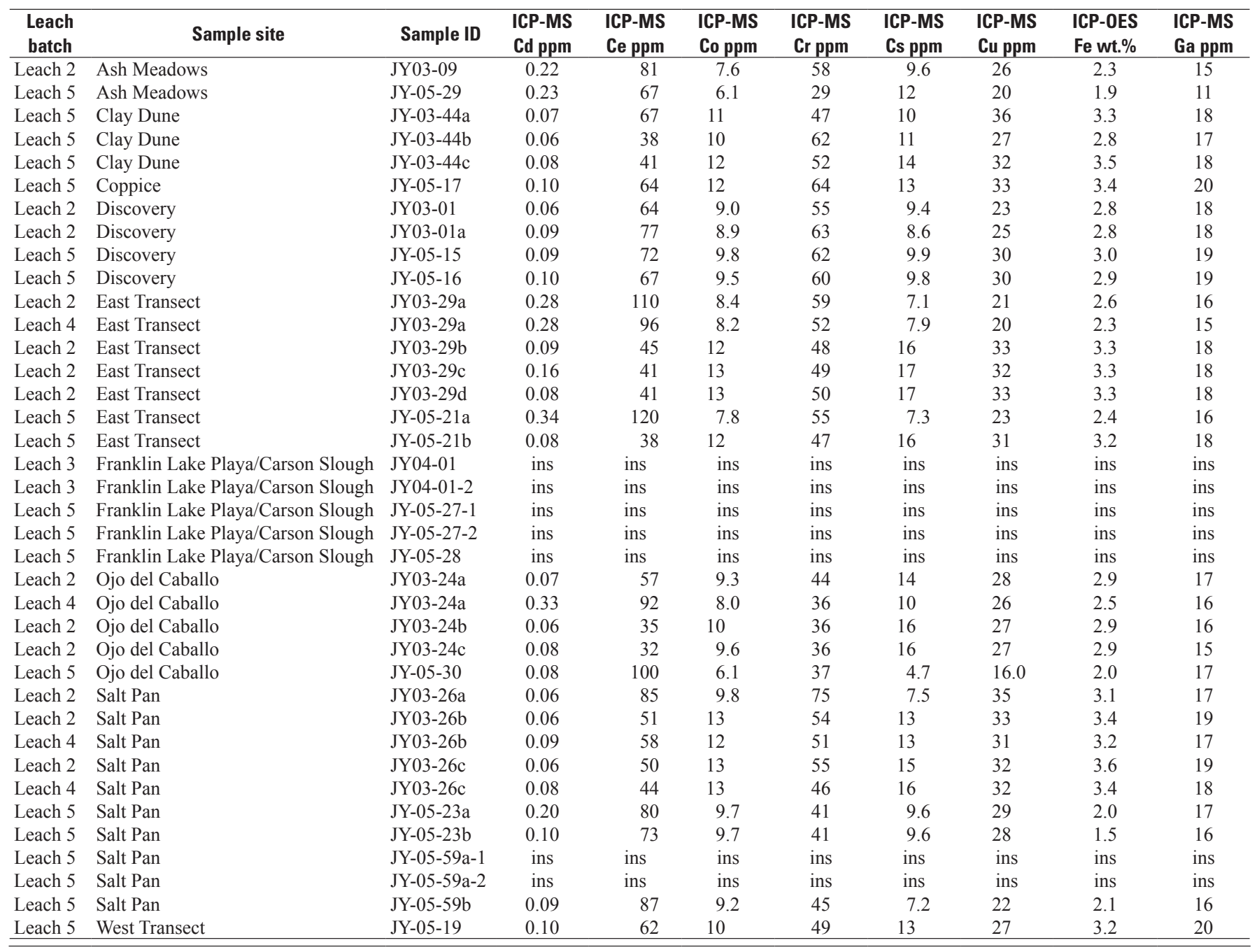


Table 12I. Composition of the residual fraction of fine fraction of grab samples. Method of determination (ICP-MS, inductively coupled plasma-mass spectrometry; ICP-OES, inductively coupled plasma-optical emission spectrometry) is listed above each element.-Continued

[Leach batch, denotes the batch in which the sample was processed and analyzed; Sample site, name of sampling site; Sample ID, unique sample identifier; Treatment, type of sample preparation; $-1,-2$, denotes duplicates; ins, insufficient sample; ppm, parts per million; wt. \%, weight percent]

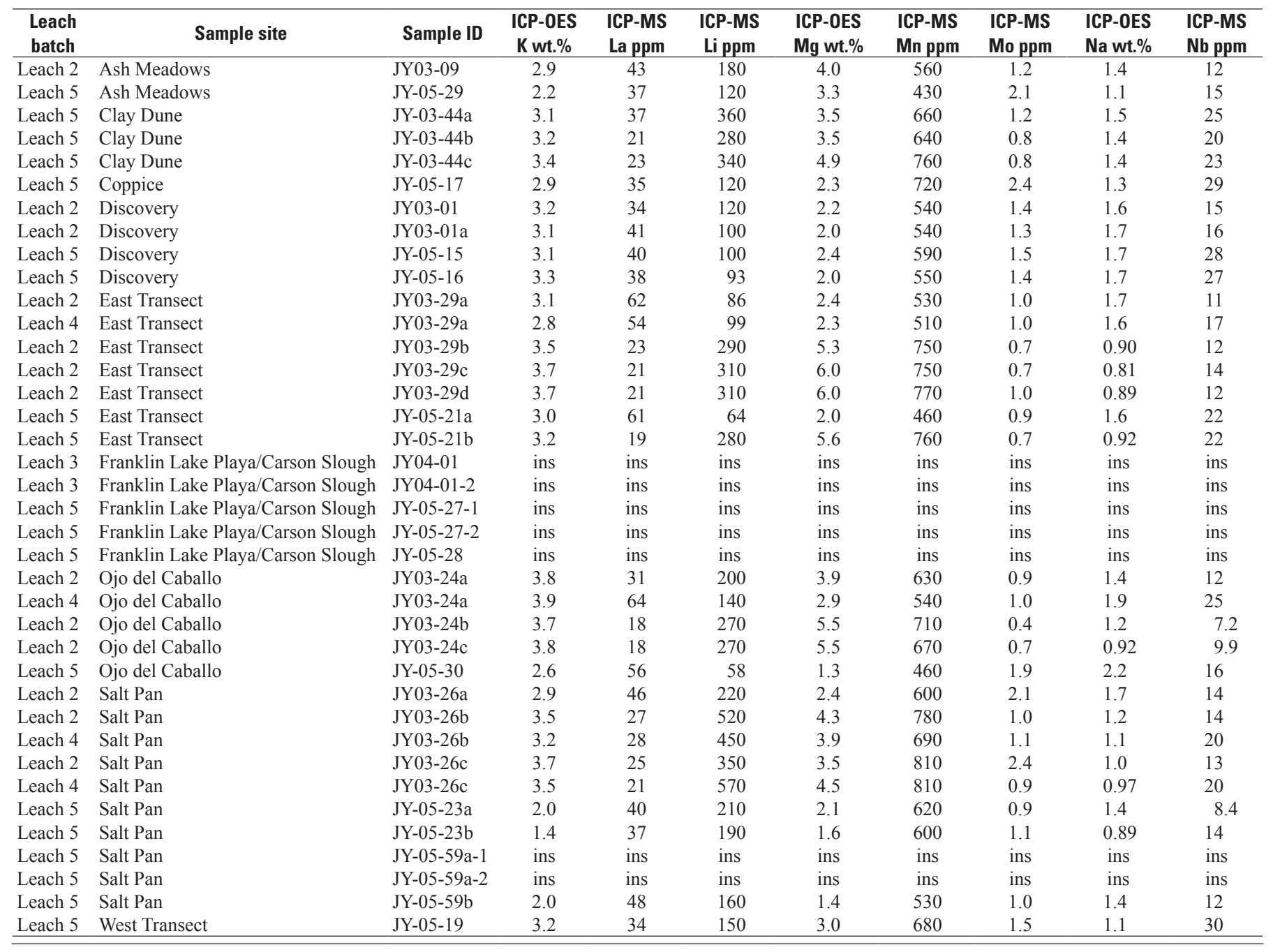


Table 12I. Composition of the residual fraction of fine fraction of grab samples. Method of determination (ICP-MS, inductively coupled plasma-mass spectrometry; ICP-OES, inductively coupled plasma-optical emission spectrometry) is listed above each element.-Continued

[Leach batch, denotes the batch in which the sample was processed and analyzed; Sample site, name of sampling site; Sample ID, unique sample identifier; Treatment, type of sample preparation; -1, -2, denotes duplicates; ins, insufficient sample; ppm, parts per million; wt.\%, weight percent]

\begin{tabular}{|c|c|c|c|c|c|c|c|c|c|c|}
\hline $\begin{array}{l}\text { Leach } \\
\text { batch }\end{array}$ & Sample site & Sample ID & $\begin{array}{l}\text { ICP-OES } \\
\text { Nd ppm }\end{array}$ & $\begin{array}{l}\text { ICP-MS } \\
\text { Ni ppm }\end{array}$ & $\begin{array}{l}\text { ICP-MS } \\
\text { P ppm }\end{array}$ & $\begin{array}{l}\text { ICP-MS } \\
\text { Pb ppm }\end{array}$ & $\begin{array}{l}\text { ICP-MS } \\
\text { Rb ppm }\end{array}$ & $\begin{array}{l}\text { ICP-MS } \\
\text { Sb ppm }\end{array}$ & $\begin{array}{l}\text { ICP-MS } \\
\text { Sc ppm }\end{array}$ & $\begin{array}{l}\text { ICP-MS } \\
\text { Sr ppm }\end{array}$ \\
\hline Leach 5 & Ash Meadows & JY-05-29 & 29 & 12 & 810 & 19 & 93 & 1.3 & 7.1 & 1,200 \\
\hline Leach 5 & Clay Dune & JY-03-44b & 16 & 21 & 230 & 14 & 150 & 1.5 & 8.3 & 190 \\
\hline Leach 5 & Clay Dune & JY-03-44c & 19 & 24 & 330 & 16 & 170 & 1.7 & 10 & 190 \\
\hline Leach 5 & Coppice & JY-05-17 & 25 & 32 & 320 & 24 & 170 & 2.0 & 12 & 260 \\
\hline Leach 2 & Discovery & JY03-01 & 23 & 19 & 320 & 20 & 150 & 1.6 & 10 & 320 \\
\hline Leach 5 & Discovery & JY-05-16 & 26 & 22 & 560 & 26 & 160 & 1.6 & 10 & 350 \\
\hline Leach 2 & East Transect & JY03-29a & 45 & 16 & 750 & 23 & 120 & 1.3 & 10 & 830 \\
\hline Leach 4 & East Transect & JY03-29a & 39 & 16 & 680 & 24 & 120 & 1.1 & 10 & 850 \\
\hline Leach 2 & East Transect & JY03-29b & 14 & 24 & 400 & 16 & 170 & 1.9 & 11 & 290 \\
\hline Leach 2 & East Transect & JY03-29c & 13 & 24 & 390 & 15 & 180 & 2.0 & 11 & 290 \\
\hline Leach 2 & East Transect & JY03-29d & 13 & 24 & 390 & 14 & 180 & 1.9 & 12 & 240 \\
\hline Leach 5 & Franklin Lake Playa/Carson Slough & JY-05-27-2 & ins & ins & ins & ins & ins & ins & ins & ins \\
\hline Leach 5 & Franklin Lake Playa/Carson Slough & JY-05-28 & ins & ins & ins & ins & ins & ins & ins & ins \\
\hline Leach 2 & Ojo del Caballo & JY03-24a & 19 & 18 & 250 & 18 & 170 & 2.6 & 9.2 & 200 \\
\hline Leach 4 & Ojo del Caballo & JY03-24a & 49 & 16 & 580 & 27 & 150 & 1.4 & 8.7 & 740 \\
\hline Leach 2 & Ojo del Caballo & JY03-24b & 11 & 19 & 150 & 12 & 170 & 1.7 & 9.2 & 96 \\
\hline Leach 2 & Ojo del Caballo & JY03-24c & 8 & 19 & 130 & 11 & 170 & 1.8 & 9.1 & 78 \\
\hline Leach 5 & Ojo del Caballo & JY-05-30 & 41 & 12 & 760 & 23 & 110 & 1.2 & 8.8 & 430 \\
\hline Leach 2 & Salt Pan & JY03-26a & 33 & 20 & 670 & 21 & 130 & 1.7 & 11 & 330 \\
\hline Leach 2 & Salt Pan & JY03-26b & 17 & 25 & 380 & 17 & 170 & 1.8 & 11 & 200 \\
\hline Leach 4 & Salt Pan & JY03-26b & 20 & 24 & 360 & 18 & 150 & 1.5 & 10 & 220 \\
\hline Leach 2 & Salt Pan & JY03-26c & 14 & 26 & 300 & 20 & 180 & 1.8 & 12 & 190 \\
\hline Leach 4 & Salt Pan & JY03-26c & 14 & 26 & 300 & 19 & 170 & 1.6 & 11 & 170 \\
\hline Leach 5 & Salt Pan & JY-05-23a & 23 & 20 & 560 & 25 & 130 & 1.4 & 9.6 & 340 \\
\hline
\end{tabular}


Table 12I. Composition of the residual fraction of fine fraction of grab samples. Method of determination (ICP-MS, inductively coupled plasma-mass spectrometry; ICP-OES, inductively coupled plasma-optical emission spectrometry) is listed above each element.-Continued

[Leach batch, denotes the batch in which the sample was processed and analyzed; Sample site, name of sampling site; Sample ID, unique sample identifier; Treatment, type of sample preparation; $-1,-2$, denotes duplicates; ins, insufficient sample; ppm, parts per million; wt.\%, weight percent]

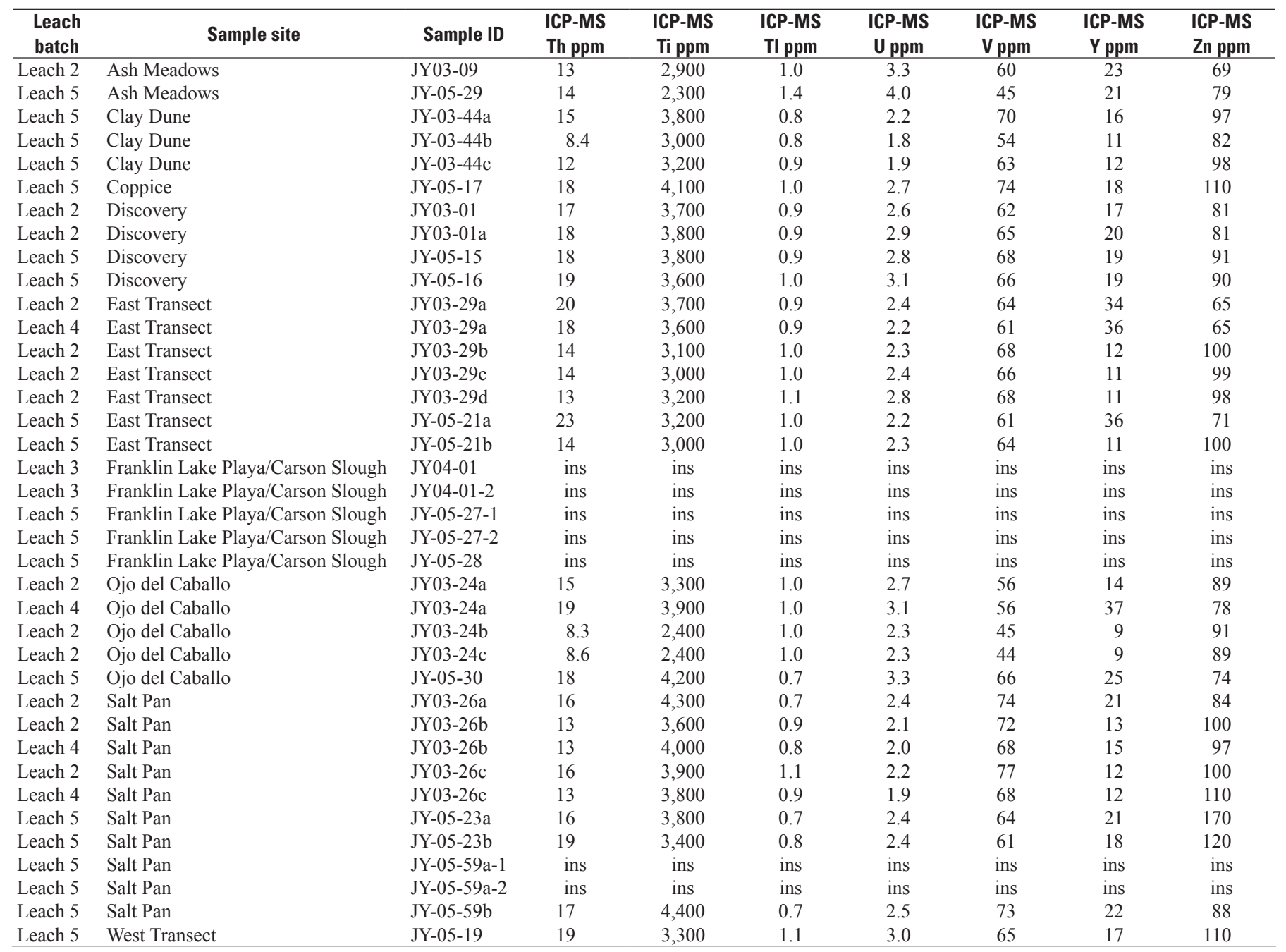


Table 13. Composition of water samples. Method of determination (ICP-MS, inductively coupled plasma-mass spectrometry; ICP-0ES, inductively coupled plasma-optical emission spectrometry; IC, ion chromatograph or IRMS, isotope ratio mass spectrometry; GSMS, gas source mass spectrometry) is listed above each element.

[Sample site, name of sampling site; Sample ID, unique sample identifier; *, well incompletely purged prior to sampling; $\mu \mathrm{g} / \mathrm{L}, \mathrm{micrograms}$ per liter; $\mathrm{mg} / \mathrm{L}$, milligrams per liter; per mil VSMOW, parts per thousand deviation from the composition of Vienna Standard Mean Ocean Water; per mil VCDT, parts per thousand deviation from the composition of Vienna Cañon Diablo Troilite]

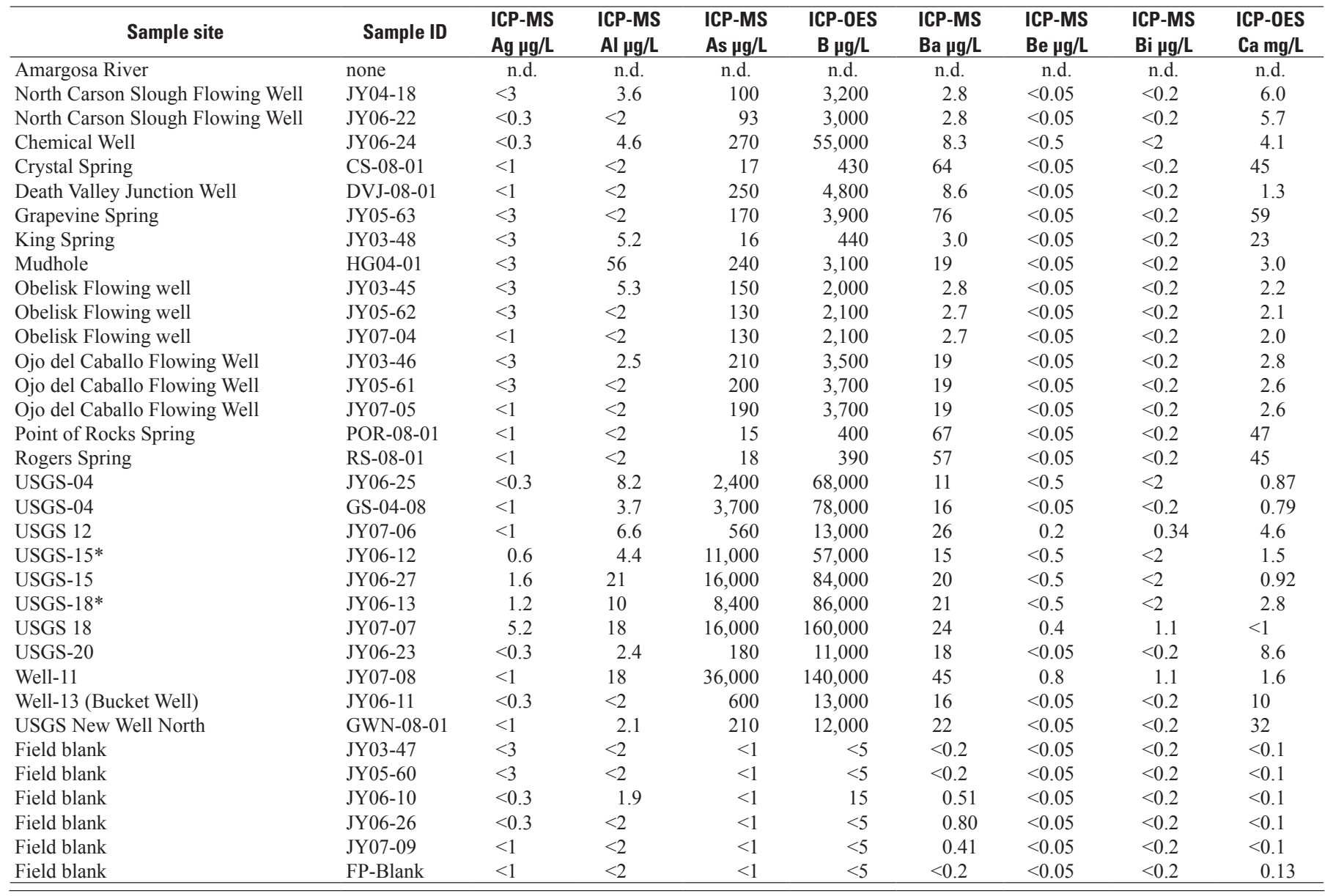


Table 13. Composition of water samples. Method of determination (ICP-MS, inductively coupled plasma-mass spectrometry; ICP-OES, inductively coupled plasma-optical emission spectrometry; IC, ion chromatograph or IRMS, isotope ratio mass spectrometry; GSMS, gas source mass spectrometry) is listed above each element.-Continued

[Sample site, name of sampling site; Sample ID, unique sample identifier; *, well incompletely purged prior to sampling; $\mu \mathrm{g} / \mathrm{L}$, micrograms per liter; mg/L, milligrams per liter; per mil VSMOW, parts per thousand deviation from the composition of Vienna Standard Mean Ocean Water; per mil VCDT, parts per thousand deviation from the composition of Vienna Cañon Diablo Troilite]

\begin{tabular}{|c|c|c|c|c|c|c|c|c|c|}
\hline Sample site & Sample ID & $\begin{array}{l}\text { ICP-MS } \\
\text { Cd } \mu \mathrm{g} / \mathrm{L}\end{array}$ & $\begin{array}{l}\text { ICP-MS } \\
\text { Ce } \mu \mathrm{g} / \mathrm{L}\end{array}$ & $\begin{array}{l}\text { ICP-MS } \\
\text { Co } \mu g / L\end{array}$ & $\begin{array}{c}\text { ICP-MS } \\
\mathrm{Cr} \mu \mathrm{g} / \mathrm{L}\end{array}$ & $\begin{array}{l}\text { ICP-MS } \\
\text { Cs } \mu \mathrm{g} / \mathrm{L}\end{array}$ & $\begin{array}{c}\text { ICP-MS } \\
\text { Cu } \mu g / L\end{array}$ & $\begin{array}{l}\text { ICP-MS } \\
\text { Dy } \mu \mathrm{g} / \mathrm{L}\end{array}$ & $\begin{array}{c}\text { ICP-MS } \\
\text { Er } \mu \mathrm{g} / \mathrm{L}\end{array}$ \\
\hline Amargosa River & none & n.d. & n.d. & n.d. & n.d. & n.d. & n.d. & n.d. & n.d. \\
\hline North Carson Slough Flowing Well & JY04-18 & 0.05 & 0.02 & $<0.02$ & 1.2 & 0.19 & 0.78 & $<0.005$ & $<0.005$ \\
\hline North Carson Slough Flowing Well & JY06-22 & 0.03 & 0.02 & $<0.02$ & $<1$ & 0.20 & 0.61 & $<0.005$ & $<0.005$ \\
\hline Chemical Well & JY06-24 & 0.82 & $<0.1$ & $<0.2$ & $<10$ & 1.0 & 7.5 & $<0.05$ & $<0.05$ \\
\hline Crystal Spring & CS-08-01 & 11 & $<0.01$ & $<0.02$ & 3.0 & 3.0 & $<0.5$ & $<0.005$ & $<0.005$ \\
\hline Death Valley Junction Well & DVJ-08-01 & 0.04 & $<0.01$ & $<0.02$ & 6.0 & 0.16 & 3.9 & $<0.005$ & $<0.005$ \\
\hline Grapevine Spring & JY05-63 & 0.05 & 0.09 & $<0.02$ & 2.9 & 0.19 & 4.8 & 0.04 & 0.03 \\
\hline King Spring & JY03-48 & 0.02 & 0.03 & 0.04 & 2.2 & 0.46 & 0.63 & $<0.005$ & $<0.005$ \\
\hline Mudhole & HG04-01 & 0.07 & 0.19 & 0.09 & 3.7 & 0.33 & 1.4 & 0.007 & 0.006 \\
\hline Obelisk Flowing Well & JY03-45 & 0.02 & 0.01 & $<0.02$ & 1.5 & 0.19 & 0.70 & $<0.005$ & $<0.005$ \\
\hline Obelisk Flowing Well & JY05-62 & 0.04 & $<0.01$ & $<0.02$ & 2.6 & 0.18 & 0.94 & $<0.005$ & $<0.005$ \\
\hline Obelisk Flowing Well & JY07-04 & 0.03 & $<0.01$ & $<0.02$ & $<1$ & 0.21 & $<0.5$ & $<0.005$ & $<0.005$ \\
\hline Ojo del Caballo Flowing Well & JY03-46 & 0.04 & $<0.01$ & 0.02 & 1.6 & 0.39 & $<0.5$ & $<0.005$ & $<0.005$ \\
\hline Ojo del Caballo Flowing Well & JY05-61 & 0.12 & $<0.01$ & $<0.02$ & 4.2 & 0.36 & 1.4 & $<0.005$ & $<0.005$ \\
\hline Ojo del Caballo Flowing Well & JY07-05 & 0.05 & $<0.01$ & $<0.02$ & 1 & 0.40 & 0.59 & $<0.005$ & $<0.005$ \\
\hline Point of Rocks Spring & POR-08-01 & $<0.02$ & $<0.01$ & $<0.02$ & 3.1 & 2.9 & $<0.5$ & $<0.005$ & $<0.005$ \\
\hline Rogers Spring & RS-08-01 & $<0.02$ & $<0.01$ & $<0.02$ & 2.9 & 3.1 & $<0.5$ & $<0.005$ & $<0.005$ \\
\hline USGS-04 & JY06-25 & 0.79 & $<0.1$ & $<0.2$ & $<10$ & 0.68 & 8.2 & $<0.05$ & $<0.05$ \\
\hline USGS-04 & GS-04-08 & 0.8 & 0.13 & 0.16 & 21 & 0.80 & 7.9 & 0.006 & $<0.005$ \\
\hline USGS 12 & JY07-06 & 0.99 & 0.02 & 0.22 & $<1$ & 2.3 & 9.6 & $<0.005$ & $<0.005$ \\
\hline USGS-15* & JY06-12 & 20 & 0.49 & 3.2 & 79 & 6.4 & 36 & $<0.05$ & $<0.05$ \\
\hline USGS-15 & JY06-27 & 20 & 2.0 & 7.5 & 210 & 10 & 50 & $<0.05$ & $<0.05$ \\
\hline USGS-18* & JY06-13 & 12 & 1.9 & 3 & 240 & 4.7 & 64 & $<0.05$ & 0.062 \\
\hline USGS 18 & JY07-07 & 17 & 7.3 & 6.7 & 450 & 8.1 & 82 & 0.04 & 0.065 \\
\hline USGS-20 & JY06-23 & 0.11 & $<0.01$ & $<0.02$ & 1 & 0.19 & 1.2 & $<0.005$ & $<0.005$ \\
\hline Well-11 & JY07-08 & 54 & 3.5 & 14 & 190 & 6.9 & 140 & 0.097 & 0.12 \\
\hline Well-13 (Bucket Well) & JY06-11 & 0.22 & 0.02 & $<0.02$ & 8 & 0.13 & 1.4 & $<0.005$ & $<0.005$ \\
\hline USGS New Well North & GWN-08-01 & 0.10 & 0.02 & 0.14 & 3.4 & 0.08 & 1.5 & 0.007 & $<0.005$ \\
\hline Field blank & JY03-47 & $<0.02$ & $<0.01$ & $<0.02$ & $<1$ & $<0.02$ & 0.66 & $<0.005$ & $<0.005$ \\
\hline Field blank & JY05-60 & $<0.02$ & $<0.01$ & $<0.02$ & $<1$ & $<0.02$ & $<0.5$ & $<0.005$ & $<0.005$ \\
\hline Field blank & JY06-10 & 0.02 & $<0.01$ & $<0.02$ & $<1$ & $<0.02$ & $<0.5$ & $<0.005$ & $<0.005$ \\
\hline Field blank & JY06-26 & $<0.02$ & $<0.01$ & $<0.02$ & $<1$ & $<0.02$ & $<0.5$ & $<0.005$ & $<0.005$ \\
\hline Field blank & JY07-09 & $<0.02$ & $<0.01$ & $<0.02$ & $<1$ & $<0.02$ & $<0.5$ & $<0.005$ & $<0.005$ \\
\hline Field blank & FP-Blank & $<0.02$ & $<0.01$ & $<0.02$ & $<1$ & $<0.02$ & 0.54 & $<0.005$ & $<0.005$ \\
\hline
\end{tabular}


Table 13. Composition of water samples. Method of determination (ICP-MS, inductively coupled plasma-mass spectrometry; ICP-0ES, inductively coupled plasma-optical emission spectrometry; IC, ion chromatograph or IRMS, isotope ratio mass spectrometry; GSMS, gas source mass spectrometry) is listed above each element.-Continued

[Sample site, name of sampling site; Sample ID, unique sample identifier; *, well incompletely purged prior to sampling; $\mu \mathrm{g} / \mathrm{L}, \mathrm{micrograms}$ per liter; $\mathrm{mg} / \mathrm{L}$, milligrams per liter; per mil VSMOW, parts per thousand deviation from the composition of Vienna Standard Mean Ocean Water; per mil VCDT, parts per thousand deviation from the composition of Vienna Cañon Diablo Troilite]

\begin{tabular}{|c|c|c|c|c|c|c|c|c|c|}
\hline Sample site & Sample ID & $\begin{array}{l}\text { ICP-MS } \\
\text { Eu } \mu g / L\end{array}$ & $\begin{array}{c}\text { ICP-0ES } \\
\mathrm{Fe} \mu \mathrm{g} / \mathrm{L}\end{array}$ & $\begin{array}{l}\text { ICP-MS } \\
\text { Ga } \mu \mathrm{g} / \mathrm{L}\end{array}$ & $\begin{array}{l}\text { ICP-MS } \\
\text { Gd } \mu \mathrm{g} / \mathrm{L}\end{array}$ & $\begin{array}{l}\text { ICP-MS } \\
\text { Ge } \mu \mathrm{g} / \mathrm{L}\end{array}$ & $\begin{array}{l}\text { ICP-MS } \\
\text { Ho } \mu \mathrm{g} / \mathrm{L}\end{array}$ & $\begin{array}{c}\text { ICP-OES } \\
\mathrm{K} \mathrm{mg/L}\end{array}$ & $\begin{array}{l}\text { ICP-MS } \\
\text { La } \mu g / L\end{array}$ \\
\hline North Carson Slough Flowing Well & JY04-18 & $<0.005$ & $<20$ & $<0.05$ & $<0.005$ & 0.6 & $<0.005$ & 17 & $<0.01$ \\
\hline North Carson Slough Flowing Well & JY06-22 & $<0.005$ & $<5$ & $<0.05$ & $<0.005$ & 0.56 & $<0.005$ & 16 & $<0.01$ \\
\hline Crystal Spring & CS-08-01 & 0.01 & $<5$ & $<0.05$ & $<0.005$ & 0.4 & $<0.005$ & 8.9 & $<0.01$ \\
\hline Death Valley Junction Well & DVJ-08-01 & $<0.005$ & $<5$ & $<0.05$ & $<0.005$ & 0.35 & $<0.005$ & 15 & $<0.01$ \\
\hline Grapevine Spring & JY05-63 & 0.01 & $<20$ & $<0.05$ & 0.04 & 0.1 & 0.01 & 52 & 0.14 \\
\hline Obelisk Flowing Well & JY03-45 & $<0.005$ & $<20$ & $<0.05$ & $<0.005$ & 0.28 & $<0.005$ & 30 & $<0.01$ \\
\hline Obelisk Flowing Well & JY05-62 & $<0.005$ & $<20$ & $<0.05$ & $<0.005$ & 0.25 & $<0.005$ & 28 & $<0.01$ \\
\hline Obelisk Flowing Well & JY07-04 & $<0.005$ & 8 & $<0.05$ & $<0.005$ & 0.28 & $<0.005$ & 29 & $<0.01$ \\
\hline Ojo del Caballo Flowing Well & JY03-46 & $<0.005$ & $<20$ & $<0.05$ & $<0.005$ & 0.64 & $<0.005$ & 56 & $<0.01$ \\
\hline Ojo del Caballo Flowing Well & JY05-61 & $<0.005$ & $<20$ & $<0.05$ & $<0.005$ & 0.53 & $<0.005$ & 57 & $<0.01$ \\
\hline Ojo del Caballo Flowing Well & JY07-05 & $<0.005$ & $<5$ & $<0.05$ & $<0.005$ & 0.66 & $<0.005$ & 57 & $<0.01$ \\
\hline USGS-15* & JY06-12 & $<0.05$ & $<20$ & $<0.5$ & $<0.05$ & $<0.5$ & $<0.05$ & 870 & $<0.1$ \\
\hline USGS-15 & JY06-27 & $<0.05$ & $<200$ & 0.74 & $<0.05$ & $<0.5$ & $<0.05$ & 1,200 & $<0.1$ \\
\hline USGS-18* & JY06-13 & $<0.05$ & $<200$ & $<0.5$ & $<0.05$ & $<0.5$ & $<0.05$ & 660 & $<0.1$ \\
\hline USGS 18 & JY07-07 & 0.01 & $<200$ & 0.37 & 0.03 & $<0.05$ & 0.007 & 1,100 & 0.03 \\
\hline USGS-20 & JY06-23 & $<0.005$ & $<5$ & $<0.05$ & $<0.005$ & 2 & $<0.005$ & 49 & $<0.01$ \\
\hline Well-11 & JY07-08 & 0.03 & $<200$ & 0.61 & $<0.005$ & $<0.05$ & 0.02 & 1,700 & 0.02 \\
\hline Well-13 (Bucket Well) & JY06-11 & $<0.005$ & $<5$ & $<0.05$ & $<0.005$ & 3 & $<0.005$ & 23 & 0.01 \\
\hline USGS New Well North & GWN-08-01 & $<0.005$ & $<5$ & $<0.05$ & 0.006 & 0.73 & $<0.005$ & 38 & $<0.01$ \\
\hline Field blank & JY03-47 & $<0.005$ & $<20$ & $<0.05$ & $<0.005$ & $<0.05$ & $<0.005$ & 0.16 & $<0.01$ \\
\hline Field blank & JY05-60 & $<0.005$ & $<20$ & $<0.05$ & $<0.005$ & $<0.05$ & $<0.005$ & $<0.1$ & $<0.01$ \\
\hline Field blank & JY06-10 & $<0.005$ & $<20$ & $<0.05$ & $<0.005$ & $<0.05$ & $<0.005$ & 0.27 & $<0.01$ \\
\hline Field blank & JY06-26 & $<0.005$ & $<20$ & $<0.05$ & $<0.005$ & $<0.05$ & $<0.005$ & $<0.1$ & $<0.01$ \\
\hline Field blank & JY07-09 & $<0.005$ & $<5$ & $<0.05$ & $<0.005$ & $<0.05$ & $<0.005$ & $<0.1$ & $<0.01$ \\
\hline
\end{tabular}


Table 13. Composition of water samples. Method of determination (ICP-MS, inductively coupled plasma-mass spectrometry; ICP-OES, inductively coupled plasma-optical emission spectrometry; IC, ion chromatograph or IRMS, isotope ratio mass spectrometry; GSMS, gas source mass spectrometry) is listed above each element.-Continued

[Sample site, name of sampling site; Sample ID, unique sample identifier; *, well incompletely purged prior to sampling; $\mu \mathrm{g} / \mathrm{L}, \mathrm{micrograms} \mathrm{per} \mathrm{liter;} \mathrm{mg/L,}$ milligrams per liter; per mil VSMOW, parts per thousand deviation from the composition of Vienna Standard Mean Ocean Water; per mil VCDT, parts per thousand deviation from the composition of Vienna Cañon Diablo Troilite]

\begin{tabular}{|c|c|c|c|c|c|c|c|c|c|}
\hline Sample site & Sample ID & $\begin{array}{l}\text { ICP-MS } \\
\text { Li } \mu \mathrm{g} / \mathrm{L}\end{array}$ & $\begin{array}{l}\text { ICP-MS } \\
\text { Lu } \mu g / L\end{array}$ & $\begin{array}{l}\text { ICP-OES } \\
\text { Mg mg/L }\end{array}$ & $\begin{array}{l}\text { ICP-MS } \\
\text { Mn } \mu \mathrm{g} / \mathrm{L}\end{array}$ & $\begin{array}{l}\text { ICP-MS } \\
\text { Mo } \mu \mathrm{g} / \mathrm{L}\end{array}$ & $\begin{array}{l}\text { ICP-OES } \\
\mathrm{Na} \mathrm{mg/L}\end{array}$ & $\begin{array}{l}\text { ICP-MS } \\
\mathrm{Nb} \mu \mathrm{g} / \mathrm{L}\end{array}$ & $\begin{array}{l}\text { ICP-MS } \\
\text { Nd } \mu g / L\end{array}$ \\
\hline North Carson Slough Flowing Well & JY04-18 & 280 & $<0.1$ & 4.4 & $<0.2$ & 27 & 370 & $<0.2$ & $<0.01$ \\
\hline North Carson Slough Flowing Well & JY06-22 & 310 & $<0.1$ & 4.3 & 0.4 & 25 & 370 & $<0.2$ & $<0.01$ \\
\hline Crystal Spring & CS-08-01 & 82 & $<0.1$ & 21 & $<0.2$ & 6.2 & 74 & $<0.2$ & $<0.01$ \\
\hline Death Valley Junction Well & DVJ-08-01 & 120 & $<0.1$ & 1.4 & 0.2 & 32 & 370 & $<0.2$ & $<0.01$ \\
\hline Grapevine Spring & JY05-63 & 310 & $<0.1$ & 59 & 12 & 21 & 570 & $<0.2$ & 0.18 \\
\hline Obelisk Flowing Well & JY03-45 & 59 & $<0.1$ & 1.8 & 1.4 & 17 & 310 & $<0.2$ & $<0.01$ \\
\hline Obelisk Flowing Well & JY05-62 & 65 & $<0.1$ & 1.8 & 1.1 & 17 & 300 & $<0.2$ & $<0.01$ \\
\hline Obelisk Flowing Well & JY07-04 & 61 & $<0.1$ & 1.7 & 1.0 & 15 & 290 & 0.28 & $<0.01$ \\
\hline Ojo del Caballo Flowing Well & JY03-46 & 36 & $<0.1$ & 3.2 & 2.5 & 47 & 610 & $<0.2$ & $<0.01$ \\
\hline Ojo del Caballo Flowing Well & JY05-61 & 44 & $<0.1$ & 3.1 & 2.5 & 44 & 600 & $<0.2$ & $<0.01$ \\
\hline Ojo del Caballo Flowing Well & JY07-05 & 36 & $<0.1$ & 3.2 & 2.2 & 40 & 650 & 0.22 & $<0.01$ \\
\hline USGS-15* & JY06-12 & 12 & $<1$ & 1.1 & 3.9 & 14,000 & 20,000 & $<2$ & $<0.1$ \\
\hline USGS-15 & JY06-27 & 13 & $<1$ & 0.44 & 5.4 & 15,000 & 28,000 & $<2$ & 0.17 \\
\hline USGS-18* & JY06-13 & 24 & $<1$ & 0.46 & 2.5 & 6,200 & 21,000 & $<2$ & $<0.1$ \\
\hline USGS 18 & JY07-07 & 11 & $<0.1$ & $<1$ & 2.5 & 12,000 & 37,000 & 1.3 & 0.05 \\
\hline USGS-20 & JY06-23 & 930 & $<0.1$ & 16 & 0.2 & 75 & 700 & 0.37 & $<0.01$ \\
\hline Well-11 & JY07-08 & 280 & 0.1 & $<1$ & 14 & 43,000 & 61,000 & 2.1 & 0.12 \\
\hline Well-13 (Bucket Well) & JY06-11 & 370 & $<0.1$ & 5.4 & 0.2 & 140 & 740 & $<0.2$ & $<0.01$ \\
\hline USGS New Well North & GWN-08-01 & 610 & $<0.1$ & 18 & 110 & 77 & 540 & $<0.2$ & $<0.01$ \\
\hline Field blank & JY03-47 & $<0.1$ & $<0.1$ & $<0.1$ & $<0.2$ & $<2$ & 0.23 & $<0.2$ & $<0.01$ \\
\hline Field blank & JY05-60 & $<0.9$ & $<0.1$ & $<0.1$ & $<0.2$ & $<2$ & $<0.1$ & $<0.2$ & $<0.01$ \\
\hline Field blank & JY06-10 & $<0.9$ & $<0.1$ & $<0.1$ & 0.6 & $<2$ & 1.3 & $<0.2$ & $<0.01$ \\
\hline Field blank & JY06-26 & $<0.9$ & $<0.1$ & $<0.1$ & 0.2 & $<2$ & $<0.1$ & $<0.2$ & $<0.01$ \\
\hline Field blank & JY07-09 & 1.2 & $<0.1$ & $<0.1$ & $<0.2$ & $<2$ & $<0.1$ & $<0.2$ & $<0.01$ \\
\hline
\end{tabular}


Table 13. Composition of water samples. Method of determination (ICP-MS, inductively coupled plasma-mass spectrometry; ICP-0ES, inductively coupled plasma-optical emission spectrometry; IC, ion chromatograph or IRMS, isotope ratio mass spectrometry; GSMS, gas source mass spectrometry) is listed above each element.-Continued

[Sample site, name of sampling site; Sample ID, unique sample identifier; *, well incompletely purged prior to sampling; $\mu \mathrm{g} / \mathrm{L}$, micrograms per liter; mg/L, milligrams per liter; per mil VSMOW, parts per thousand deviation from the composition of Vienna Standard Mean Ocean Water; per mil VCDT, parts per thousand deviation from the composition of Vienna Cañon Diablo Troilite]

\begin{tabular}{|c|c|c|c|c|c|c|c|c|c|}
\hline Sample site & Sample ID & $\begin{array}{l}\text { ICP-MS } \\
\mathrm{Ni} \mu \mathrm{g} / \mathrm{L}\end{array}$ & $\begin{array}{c}\text { ICP-MS } \\
P \mu g / L\end{array}$ & $\begin{array}{l}\text { ICP-MS } \\
\mathrm{Pb} \mu \mathrm{g} / \mathrm{L}\end{array}$ & $\begin{array}{l}\text { ICP-MS } \\
\operatorname{Pr} \mu \mathrm{g} / \mathrm{L}\end{array}$ & $\begin{array}{l}\text { ICP-MS } \\
\mathrm{Rb} \mu \mathrm{g} / \mathrm{L}\end{array}$ & $\begin{array}{l}\text { ICP-MS } \\
\text { Sb } \mu g / L\end{array}$ & $\begin{array}{l}\text { ICP-MS } \\
\text { Sc } \mu g / L\end{array}$ & $\begin{array}{l}\text { ICP-MS } \\
\text { Se } \mu \mathrm{g} / \mathrm{L}\end{array}$ \\
\hline North Carson Slough Flowing Well & JY04-18 & $<0.4$ & 0.03 & $<0.05$ & $<0.01$ & 20 & 0.50 & 11 & 2.9 \\
\hline North Carson Slough Flowing Well & JY06-22 & $<0.4$ & $<0.01$ & 0.51 & $<0.01$ & 19 & 0.80 & 4.4 & 1.7 \\
\hline Crystal Spring & CS-08-01 & 0.6 & $<0.01$ & $<0.05$ & $<0.01$ & 15 & $<0.3$ & 2.6 & 1.2 \\
\hline Death Valley Junction Well & DVJ-08-01 & $<0.4$ & 0.04 & 0.2 & $<0.01$ & 14 & 0.30 & 1.1 & 2.3 \\
\hline Grapevine Spring & JY05-63 & $<0.4$ & $<0.01$ & $<0.05$ & 0.03 & 60 & $<0.3$ & 6.0 & 11 \\
\hline Obelisk Flowing Well & JY03-45 & $<0.4$ & 0.06 & 0.06 & $<0.01$ & 20 & 0.72 & 3.2 & nd \\
\hline Obelisk Flowing Well & JY05-62 & $<0.4$ & 0.04 & $<0.05$ & $<0.01$ & 21 & 0.77 & 7.0 & 2.2 \\
\hline Obelisk Flowing Well & JY07-04 & $<0.4$ & 0.05 & $<0.05$ & $<0.01$ & 19 & 1.0 & 3.2 & 1.8 \\
\hline Ojo del Caballo Flowing Well & JY03-46 & $<0.4$ & 0.09 & $<0.05$ & $<0.01$ & 39 & $<0.3$ & 2.9 & nd \\
\hline Ojo del Caballo Flowing Well & JY05-61 & $<0.4$ & 0.07 & $<0.05$ & $<0.01$ & 38 & $<0.3$ & 8.5 & 8.3 \\
\hline Ojo del Caballo Flowing Well & JY07-05 & $<0.4$ & 0.06 & 0.2 & $<0.01$ & 36 & $<0.3$ & 2.8 & 4.2 \\
\hline USGS-15* & JY06-12 & $<4$ & 4.6 & 22 & $<0.1$ & 510 & 7.2 & 9.0 & 410 \\
\hline USGS-15 & JY06-27 & 5.4 & 13 & 9.7 & $<0.1$ & 710 & 8.7 & 16 & 420 \\
\hline USGS-18* & JY06-13 & 4.2 & 12 & 150 & $<0.1$ & 370 & 12 & 8.0 & 280 \\
\hline USGS 18 & JY07-07 & 6.4 & 30 & 150 & 0.02 & 560 & 21 & 8.4 & 680 \\
\hline USGS-20 & JY06-23 & $<0.4$ & 0.03 & 0.79 & $<0.01$ & 25 & 0.58 & 7.4 & 3.0 \\
\hline Well-11 & JY07-08 & 15 & 47 & 230 & 0.02 & 810 & 44 & 15 & 1,800 \\
\hline Well-13 (Bucket Well) & JY06-11 & $<0.4$ & 0.05 & 1.2 & $<0.01$ & 34 & 1.1 & 5.3 & 4.7 \\
\hline USGS New Well North & GWN-08-01 & $<0.4$ & 0.3 & 0.2 & $<0.01$ & 18 & 0.61 & 5.1 & 3.6 \\
\hline Field blank & JY03-47 & $<0.4$ & $<0.01$ & 0.09 & $<0.01$ & 0.15 & $<0.3$ & $<0.6$ & nd \\
\hline Field blank & JY05-60 & $<0.4$ & $<0.01$ & $<0.05$ & $<0.01$ & $<0.01$ & $<0.3$ & $<0.6$ & $<1$ \\
\hline Field blank & JY06-10 & 0.4 & $<0.01$ & 0.2 & $<0.01$ & 0.1 & $<0.3$ & $<0.6$ & $<1$ \\
\hline Field blank & JY06-26 & $<0.4$ & $<0.01$ & 0.1 & $<0.01$ & 0.02 & $<0.3$ & $<0.6$ & $<1$ \\
\hline Field blank & JY07-09 & $<0.4$ & $<0.01$ & 0.05 & $<0.01$ & 0.17 & $<0.3$ & $<0.6$ & $<1$ \\
\hline
\end{tabular}


Table 13. Composition of water samples. Method of determination (ICP-MS, inductively coupled plasma-mass spectrometry; ICP-OES, inductively coupled plasma-optical emission spectrometry; IC, ion chromatograph or IRMS, isotope ratio mass spectrometry; GSMS, gas source mass spectrometry) is listed above each element.-Continued

[Sample site, name of sampling site; Sample ID, unique sample identifier; *, well incompletely purged prior to sampling; $\mu \mathrm{g} / \mathrm{L}$, micrograms per liter; mg/L, milligrams per liter; per mil VSMOW, parts per thousand deviation from the composition of Vienna Standard Mean Ocean Water; per mil VCDT, parts per thousand deviation from the composition of Vienna Cañon Diablo Troilite]

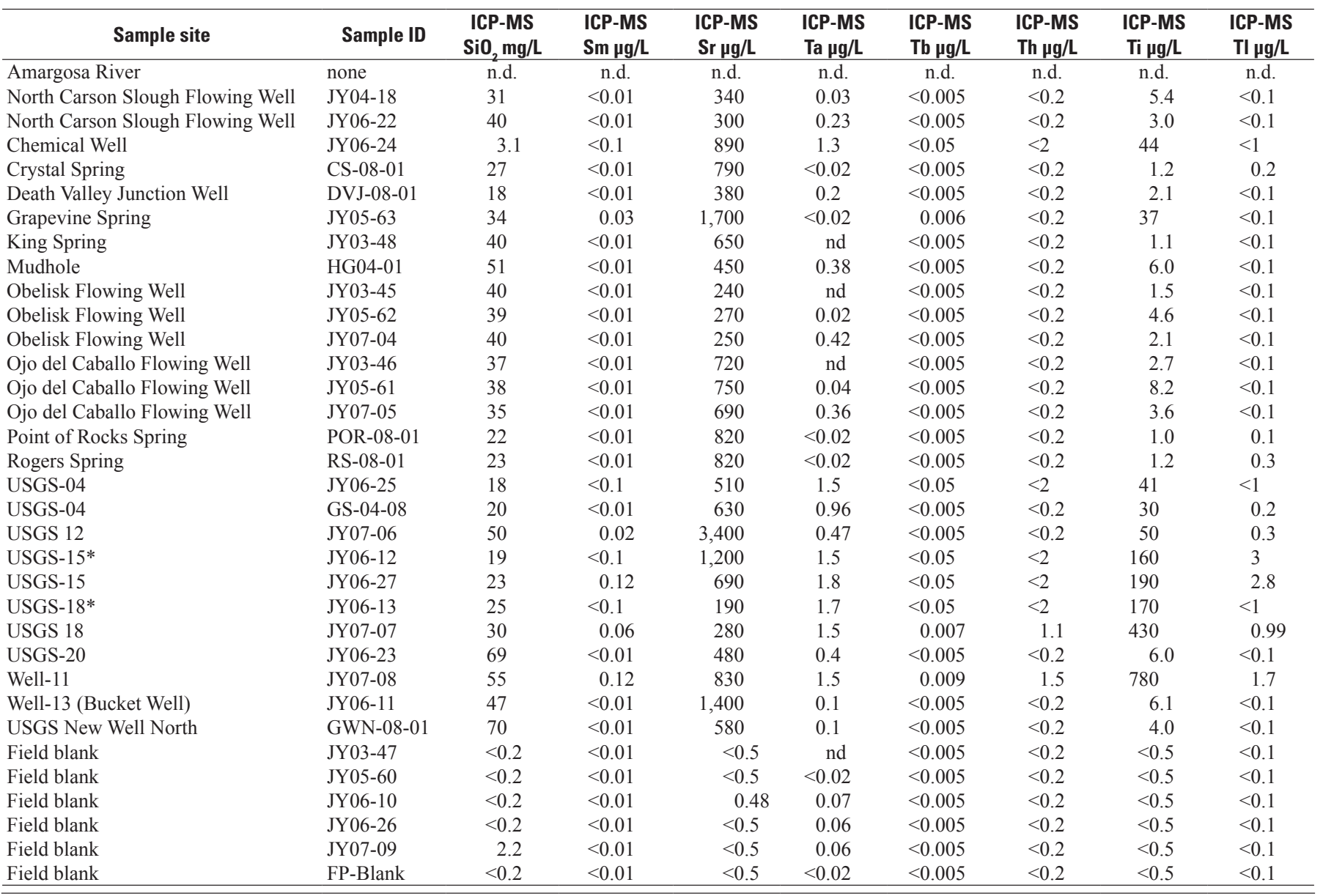


Table 13. Composition of water samples. Method of determination (ICP-MS, inductively coupled plasma-mass spectrometry; ICP-0ES, inductively coupled plasma-optical emission spectrometry; IC, ion chromatograph or IRMS, isotope ratio mass spectrometry; GSMS, gas source mass spectrometry) is listed above each element.-Continued

[Sample site, name of sampling site; Sample ID, unique sample identifier; *, well incompletely purged prior to sampling; $\mu \mathrm{g} / \mathrm{L}$, micrograms per liter; mg/L, milligrams per liter; per mil VSMOW, parts per thousand deviation from the composition of Vienna Standard Mean Ocean Water; per mil VCDT, parts per thousand deviation from the composition of Vienna Cañon Diablo Troilite]

\begin{tabular}{|c|c|c|c|c|c|c|c|c|c|c|}
\hline Sample site & Sample ID & $\begin{array}{l}\text { ICP-MS } \\
\mathrm{Tm} \mu \mathrm{g} / \mathrm{L}\end{array}$ & $\begin{array}{c}\text { ICP-MS } \\
\text { U } \mu \mathrm{g} / \mathrm{L}\end{array}$ & $\begin{array}{c}\text { ICP-MS } \\
\text { V } \mu g / L\end{array}$ & $\begin{array}{l}\text { ICP-MS } \\
W_{\mu g / L}\end{array}$ & $\begin{array}{c}\text { ICP-MS } \\
Y_{\mu g / L}\end{array}$ & $\begin{array}{l}\text { ICP-MS } \\
\text { Yb } \mu g / L\end{array}$ & $\begin{array}{l}\text { ICP-MS } \\
\text { Zn } \mu g / L\end{array}$ & $\begin{array}{l}\text { ICP-MS } \\
\mathrm{Zr} \mu \mathrm{g} / \mathrm{L}\end{array}$ & $\begin{array}{c}\text { IC } \\
\text { Cl- }^{-} \mathrm{mg} / \mathrm{L} \\
\end{array}$ \\
\hline North Carson Slough Flowing Well & JY04-18 & $<0.005$ & 9.9 & 11 & 1.9 & 0.03 & $<0.005$ & 2.6 & 0.2 & 160 \\
\hline Crystal Spring & CS-08-01 & $<0.005$ & 2.1 & 1.8 & $<0.5$ & 0.02 & $<0.005$ & 0.8 & $<0.2$ & 26 \\
\hline Death Valley Junction Well & DVJ-08-01 & $<0.005$ & 5.7 & 22 & 6.4 & 0.03 & $<0.005$ & 4.5 & $<0.2$ & 77 \\
\hline Grapevine Spring & JY05-63 & $<0.005$ & 17 & 7.5 & 0.85 & 0.34 & 0.03 & 3.4 & $<0.2$ & 230 \\
\hline Obelisk Flowing Well & JY03-45 & $<0.005$ & 5.2 & 16 & nd & 0.03 & 0.007 & 5.5 & 0.3 & 49 \\
\hline Obelisk Flowing Well & JY05-62 & $<0.005$ & 4.6 & 15 & 12 & 0.03 & $<0.005$ & $<0.5$ & $<0.2$ & 54 \\
\hline Obelisk Flowing Well & JY07-04 & $<0.005$ & 5.0 & 15 & 9.6 & 0.03 & $<0.005$ & 1.4 & 0.51 & 49 \\
\hline Ojo del Caballo Flowing Well & JY03-46 & $<0.005$ & 15 & $<0.5$ & nd & 0.06 & $<0.005$ & $<0.5$ & 0.2 & 420 \\
\hline Ojo del Caballo Flowing Well & JY05-61 & $<0.005$ & 14 & 0.5 & 12 & 0.02 & $<0.005$ & 0.8 & 0.2 & 460 \\
\hline Ojo del Caballo Flowing Well & JY07-05 & $<0.005$ & 16 & $<0.5$ & 10 & 0.03 & $<0.005$ & 0.6 & $<0.2$ & 420 \\
\hline USGS-15* & JY06-12 & $<0.05$ & 2,900 & 420 & 190 & $<0.1$ & 0.07 & 42 & $<2$ & 19,000 \\
\hline USGS-15 & JY06-27 & $<0.05$ & 4,300 & 580 & 330 & 0.16 & 0.09 & 53 & $<2$ & 26,000 \\
\hline USGS-18* & JY06-13 & $<0.05$ & 2,300 & 410 & 190 & 0.24 & 0.08 & 59 & $<2$ & 14,000 \\
\hline USGS 18 & JY07-07 & 0.02 & 4,000 & 710 & 310 & 0.39 & 0.09 & 78 & 1.9 & 29,000 \\
\hline USGS-20 & JY06-23 & $<0.005$ & 6.0 & 7.0 & $<0.5$ & $<0.01$ & $<0.005$ & 5.2 & 0.3 & 230 \\
\hline Well-11 & JY07-08 & 0.057 & 10,000 & 1,200 & 680 & 0.56 & 0.28 & 93 & 25 & 54,000 \\
\hline Well-13 (Bucket Well) & JY06-11 & $<0.005$ & 23 & 48 & 4.5 & 0.04 & 0.005 & 3.4 & $<0.2$ & 500 \\
\hline USGS New Well North & GWN-08-01 & $<0.005$ & 2.1 & 1.1 & 2.0 & 0.06 & 0.009 & 0.9 & $<0.2$ & 260 \\
\hline Field blank & JY03-47 & $<0.005$ & $<0.1$ & $<0.5$ & nd & $<0.01$ & $<0.005$ & 5.6 & $<0.2$ & $<0.08$ \\
\hline Field blank & JY05-60 & $<0.005$ & $<0.1$ & $<0.5$ & $<0.5$ & $<0.01$ & $<0.005$ & $<0.5$ & $<0.2$ & $<0.08$ \\
\hline Field blank & JY06-10 & $<0.005$ & $<0.1$ & $<0.5$ & $<0.5$ & $<0.01$ & $<0.005$ & 7.5 & $<0.2$ & $<0.08$ \\
\hline Field blank & JY06-26 & $<0.005$ & $<0.1$ & $<0.5$ & $<0.5$ & $<0.01$ & $<0.005$ & 6.6 & $<0.2$ & 0.15 \\
\hline Field blank & JY07-09 & $<0.005$ & $<0.1$ & $<0.5$ & $<0.5$ & $<0.01$ & $<0.005$ & 1.4 & $<0.2$ & $<0.08$ \\
\hline
\end{tabular}


Table 13. Composition of water samples. Method of determination (ICP-MS, inductively coupled plasma-mass spectrometry; ICP-OES, inductively coupled plasma-optical emission spectrometry; IC, ion chromatograph or IRMS, isotope ratio mass spectrometry; GSMS, gas source mass spectrometry) is listed above each element.-Continued

[Sample site, name of sampling site; Sample ID, unique sample identifier; *, well incompletely purged prior to sampling; $\mu \mathrm{g} / \mathrm{L}, \mathrm{micrograms} \mathrm{per} \mathrm{liter;} \mathrm{mg/L,}$ milligrams per liter; per mil VSMOW, parts per thousand deviation from the composition of Vienna Standard Mean Ocean Water; per mil VCDT, parts per thousand deviation from the composition of Vienna Cañon Diablo Troilite]

\begin{tabular}{|c|c|c|c|c|c|c|c|c|}
\hline Sample site & Sample ID & $\begin{array}{c}\text { IC } \\
F^{-}-\mathrm{mg} / \mathrm{L}\end{array}$ & $\begin{array}{c}\text { IC } \\
\mathrm{NO}_{3}-\mathrm{mg} / \mathrm{L}\end{array}$ & $\begin{array}{c}\text { IC } \\
\mathrm{SO}_{4}{ }^{2-} \mathrm{mg} / \mathrm{L}\end{array}$ & $\begin{array}{c}\text { IRMS } \delta \mathrm{D}-\mathrm{H}_{2} \mathrm{O} \\
\text { (per mil VSMOW) }\end{array}$ & $\begin{array}{c}\text { IRMS } \delta^{18} \mathbf{0}-\mathrm{H}_{2} \mathbf{O} \\
\text { (per mil VSMOW) }\end{array}$ & $\begin{array}{l}\text { GSMS } \delta^{34} \text { S-SO } \\
\text { (per mil VCDT) }\end{array}$ & $\begin{array}{c}\text { GSMS } \delta^{18} \mathrm{O}^{-\mathrm{SO}_{4}} \\
\text { (per mil VSMOW) }\end{array}$ \\
\hline North Carson Slough Flowing Well & JY04-18 & 0.4 & $<0.08$ & 300 & n.d. & n.d. & n.d. & n.d. \\
\hline North Carson Slough Flowing Well & JY06-22 & 2.6 & 1.3 & 300 & n.d. & n.d. & 19.8 & 6.7 \\
\hline Crystal Spring & CS-08-01 & 2.0 & 9.1 & 93 & n.d. & n.d. & 16.0 & 4 \\
\hline Death Valley Junction Well & DVJ-08-01 & 6.7 & 18 & 210 & n.d. & n.d. & 19.3 & 7.4 \\
\hline Grapevine Spring & JY05-63 & 1.8 & 1.1 & 1,100 & n.d. & n.d. & n.d. & n.d. \\
\hline Obelisk Flowing Well & JY03-45 & 4.9 & $<0.08$ & 150 & -13.5 & -103 & n.d. & n.d. \\
\hline Obelisk Flowing Well & JY05-62 & 6.5 & $<0.08$ & 150 & n.d. & n.d. & 20.4 & 5.1 \\
\hline Obelisk Flowing Well & JY07-04 & 5.0 & $<0.08$ & 140 & n.d. & n.d. & n.d. & n.d. \\
\hline Ojo del Caballo Flowing Well & JY03-46 & 6.4 & $<0.08$ & 210 & n.d. & n.d. & n.d. & n.d. \\
\hline Ojo del Caballo Flowing Well & JY05-61 & 7.6 & $<0.08$ & 250 & -13.1 & -102 & n.d. & n.d. \\
\hline Ojo del Caballo Flowing Well & JY07-05 & 5.8 & $<0.08$ & 230 & nd & n.d. & 18.7 & 5.6 \\
\hline Point of Rocks Spring & POR-08-01 & 1.6 & 9.3 & 82 & n.d. & n.d. & 16.0 & 4.2 \\
\hline USGS-15* & JY06-12 & 18 & 360 & 13,000 & n.d. & n.d. & n.d. & n.d. \\
\hline USGS-15 & JY06-27 & 26 & 550 & 17,000 & n.d. & n.d. & 15.8 & 7.4 \\
\hline USGS-18* & JY06-13 & $<1$ & 510 & 9,000 & n.d. & n.d. & n.d. & n.d. \\
\hline USGS 18 & JY07-07 & 6.0 & 1,100 & 24,000 & n.d. & n.d. & 16.8 & 7.8 \\
\hline USGS-20 & JY06-23 & 5.2 & $<0.08$ & 460 & n.d. & n.d. & 14.9 & 7.3 \\
\hline Well-11 & JY07-08 & 42 & 2,700 & 42,000 & n.d. & n.d. & 12.1 & n.d. \\
\hline Well-13 (Bucket Well) & JY06-11 & 2.3 & 2.0 & 550 & n.d. & n.d. & 11.4 & 5.8 \\
\hline USGS New Well North & GWN-08-01 & 8.5 & $<0.08$ & 420 & n.d. & n.d. & 13.8 & 8.8 \\
\hline Field blank & JY03-47 & $<0.08$ & $<0.08$ & $<1.6$ & n.d. & n.d. & n.d. & n.d. \\
\hline Field blank & JY05-60 & $<0.08$ & $<0.08$ & $<1.6$ & n.d. & n.d. & n.d. & n.d. \\
\hline Field blank & JY06-10 & $<0.08$ & $<0.08$ & $<1.6$ & n.d. & n.d. & n.d. & n.d. \\
\hline Field blank & JY06-26 & $<0.08$ & 1.6 & $<1.6$ & n.d. & n.d. & n.d. & n.d. \\
\hline Field blank & JY07-09 & $<0.08$ & $<0.08$ & $<1.6$ & n.d. & n.d. & n.d. & n.d. \\
\hline
\end{tabular}


Table 14A. Electrical conductivity measurements of bulk ager hole samples.

[Sample site, name of sampling site; Sample ID, unique sample identifier; $\mu \mathrm{S} / \mathrm{cm}$, millisiemens per centimeter]

\begin{tabular}{|c|c|c|c|c|c|}
\hline Site name & Sample ID & $\begin{array}{c}\text { Specific conductance } \\
\mu \mathrm{S} / \mathrm{cm}\end{array}$ & Site name & Sample ID & $\begin{array}{c}\text { Specific conductance } \\
\mu \mathrm{S} / \mathrm{cm}\end{array}$ \\
\hline Clay Dune & HG09-01/1-5 & 7.5 & Discovery & DS04-07/87-104 & 0.38 \\
\hline Clay Dune & HG09-01/10-20 & 7.8 & Discovery & DS04-07/118-131 & 0.39 \\
\hline Clay Dune & HG09-01/20-30 & 1.7 & Discovery & DS04-07/131-142 & 0.34 \\
\hline Clay Dune & HG09-01/30-40 & 2.6 & Discovery & DS04-07/142-157 & 0.29 \\
\hline Coppice & HG09-04/0-1 & 7.1 & Discovery & DS04-07b/0-1 & 17 \\
\hline Coppice & HG09-04/1-5 & 6.2 & Discovery & DS04-07b/1-2.5 & 12 \\
\hline Coppice & HG09-04/5-10 & 8.1 & Discovery & DS04-07b/2.5-5 & 8.2 \\
\hline Coppice & HG09-04/10-20 & 7.2 & Discovery & HG09-02/0-1 & 4.5 \\
\hline Coppice & HG09-04/20-32 & 2.9 & Discovery & HG09-02/1-5 & 6.5 \\
\hline Coppice & HG09-04/32-45 & 2.2 & Discovery & HG09-02/5-10 & 12.3 \\
\hline Coppice & HG09-04/153-177 & 0.96 & Discovery & HG09-02/80-89 & 0.38 \\
\hline Coppice & HG09-04/205-214 & 0.61 & Discovery & HG09-02/98-110 & 0.35 \\
\hline Coppice & HG09-04/242-251 & 0.52 & East Transect & HG07-04/0-1 & 0.96 \\
\hline Coppice & HG09-04/268-275 & 0.51 & East Transect & HG07-04/1-5 & 2.0 \\
\hline Coppice & HG09-04/275-284 & 0.43 & East Transect & HG07-04/5-10 & 8.0 \\
\hline Coppice & HG09-04/284-293 & 0.51 & East Transect & HG07-04/10-20 & 3.5 \\
\hline Coppice & HG09-04/326-339 & 0.33 & East Transect & HG07-04/20-30 & 4.2 \\
\hline Coppice & HG09-04/352-365 & 0.36 & East Transect & HG07-04/30-40 & 3.1 \\
\hline Coppice & HG09-04/365-380 & 0.44 & East Transect & HG07-04/40-50 & 2.7 \\
\hline Coppice & HG09-04/380-404 & 0.37 & East Transect & HG07-04/50-60 & 2.7 \\
\hline Coppice & HG09-04/404-430 & 0.39 & East Transect & HG07-04/60-70 & 2.7 \\
\hline Coppice & СРAH04-09/0-0.5 & 25 & East Transect & HG07-04/70-80 & 2.5 \\
\hline Coppice & СРАН04-09/0.5-3 & 4.7 & East Transect & HG07-04/80-90 & 2.2 \\
\hline Discovery & DS04-07/0-2 & 16 & East Transect & HG07-04/169-177 & 0.87 \\
\hline Discovery & DS04-07/2-8 & 8.5 & East Transect & HG07-04/177-184 & 0.88 \\
\hline Discovery & DS04-07/8-14 & 2.5 & East Transect & HG07-04/184-193 & 1.2 \\
\hline Discovery & DS04-07/14-20 & 2.0 & East Transect & HG07-04/193-203 & 1.4 \\
\hline Discovery & DS04-07/20-30 & 1.8 & East Transect & HG07-04/203-217 & 1.4 \\
\hline Discovery & DS04-07/30-35 & 1.1 & East Transect & HG07-04/217-228 & 0.71 \\
\hline Discovery & DS04-07/35-48 & 0.67 & East Transect & HG07-04/228-237 & 0.82 \\
\hline Discovery & DS04-07/48-57 & 0.72 & East Transect & HG07-04/237-248 & 0.89 \\
\hline Discovery & DS04-07/57-68 & 0.47 & East Transect & HG07-04/248-258 & 1.1 \\
\hline Discovery & DS04-07/68-82 & 0.45 & East Transect & HG07-04/258-266 & 1.3 \\
\hline
\end{tabular}


Table 14A. Electrical conductivity measurements of bulk ager hole samples.-Continued

[Sample site, name of sampling site; Sample ID, unique sample identifier; $\mu \mathrm{S} / \mathrm{cm}$, millisiemens per centimeter]

\begin{tabular}{|c|c|c|c|c|c|}
\hline Site name & Sample ID & $\begin{array}{c}\text { Specific conductance } \\
\mu \mathrm{S} / \mathrm{cm}\end{array}$ & Site name & Sample ID & $\begin{array}{c}\text { Specific conductance } \\
\mu \mathrm{S} / \mathrm{cm}\end{array}$ \\
\hline East Transect & FPAH06-08/0-1 & 0.72 & East Transect & FPAH02-09/20-25 & nd \\
\hline East Transect & FPAH06-08/2-5 & 2.4 & East Transect & FPAH02-09/30-35 & nd \\
\hline East Transect & FPAH06-08/5-10 & 1.4 & East Transect & FPAH02-09/35-40 & nd \\
\hline East Transect & FPAH06-08/10-20 & 2.4 & East Transect & FPAH02-09/40-45 & nd \\
\hline East Transect & FPAH06-08/30-35 & 3.2 & East Transect & FPAH04-09/0-1 & 0.75 \\
\hline East Transect & FPAH06-08/35-40 & 3.2 & East Transect & FPAH04-09/1-3 & 1.2 \\
\hline East Transect & FPAH06-08/40-45 & 3.3 & East Transect & FPAH04-09/3-5.5 & 2.3 \\
\hline East Transect & FPAH06-08/45-48 & 3.1 & East Transect & FPAH04-09/5-8 & 3.2 \\
\hline East Transect & FPAH06-08/48-52 & 3.2 & East Transect & FPAH04-09/8-11 & 2.8 \\
\hline East Transect & HG08-01/0-1 & 0.54 & East Transect & FPAH04-09/11-15 & 2.9 \\
\hline East Transect & HG08-01/15-20 & 4.2 & East Transect & FPAH04-09/35-40 & 3.0 \\
\hline East Transect & HG08-01/20-25 & 3.6 & Salt Pan & SPAH04-07/0-2 & 19 \\
\hline East Transect & HG08-01/25-30 & 3.6 & Salt Pan & SPAH04-07/2-5 & 3.0 \\
\hline East Transect & HG08-01/30-35 & 3.4 & Salt Pan & SPAH04-07/5-10 & 1.1 \\
\hline East Transect & HG08-01/35-40 & 3.0 & Salt Pan & SPAH04-07/10-20 & 0.90 \\
\hline East Transect & HG08-01/40-45 & 2.7 & Salt Pan & SPAH04-07/20-30 & 1.1 \\
\hline East Transect & HG08-01/45-50 & 2.6 & Salt Pan & SPAH04-07/30-45 & 0.52 \\
\hline East Transect & FPAH02-09/0-1 & nd & Salt Pan & SPAH04-07/45-58 & 0.63 \\
\hline East Transect & FPAH02-09/1-2.5 & nd & Salt Pan & SPAH04-07/58-69 & 0.42 \\
\hline East Transect & FPAH02-09/2.5-4 & nd & Salt Pan & SPAH04-07/69-84 & 0.57 \\
\hline East Transect & FPAH02-09/4-10 & nd & Salt Pan & SPAH04-07/84-105 & 0.47 \\
\hline
\end{tabular}

Table 14B. Electrical conductivity measurements of bulk grab samples.

[Sample site, name of sampling site; Sample ID, unique sample identifier; $\mu \mathrm{S} / \mathrm{cm}$, millisiemens per centimeter]

\begin{tabular}{llc}
\hline \multicolumn{1}{c}{ Site name } & Sample ID & $\begin{array}{r}\text { Specific conductance } \\
\boldsymbol{\mu S / c m}\end{array}$ \\
\hline Between GS-18 and East Transect & JY07-12a & 420 \\
Between GS-18 and East Transect & JY07-12b & 1,560 \\
Coppice reference & FP-cop-ref & 8,350 \\
Discovery & JY07-09 & 16,100 \\
Near GS-15 & JY07-10a & 850 \\
Near GS-15 & JY07-10b & 4,760 \\
Near GS-18 & JY07-11a & 620 \\
Near GS-18 & JY07-11b & 1,420 \\
North Carson Slough, North of Hwy & JY07-13 & 24,300 \\
North Carson Slough, North of Hwy & JY07-13d & 24,100 \\
North Carson Slough, North of Hwy & JY07-14 & 27,300 \\
\hline
\end{tabular}


Table 15. Mineralogical data of auger hole and grab samples determined by X-ray diffraction.

[Sample site, name of sampling site; Sample ID, unique sample identifier; Treatment, type of sample preparation; Size, size fraction analyzed; Solid, unaltered bulk material; Slurry, water and sediment mixture; DI residue, remaining material after DI leaching; Clay, clay separates; Final residue, remaining material after nitric acid leaching; X, mineral is detected; I, illite; K, kaolinite; Se, sepiolite; Chl, chlorite; S, smectite/montmorillonite/beidellite; C, clinoptilolite; H, heulandite; M, mordentie; A, analcime; Z, unknown zeolite; P, plagioclase; K-spar, potassium feldspar; Y, yeelimite; Aph, aphthitalite; G, gaylussite; Ar, aragonite; $\mathrm{Mg}$, magnesite; $\mathrm{Th}$, thermonatrite; $\mathrm{N}$, norsethite]

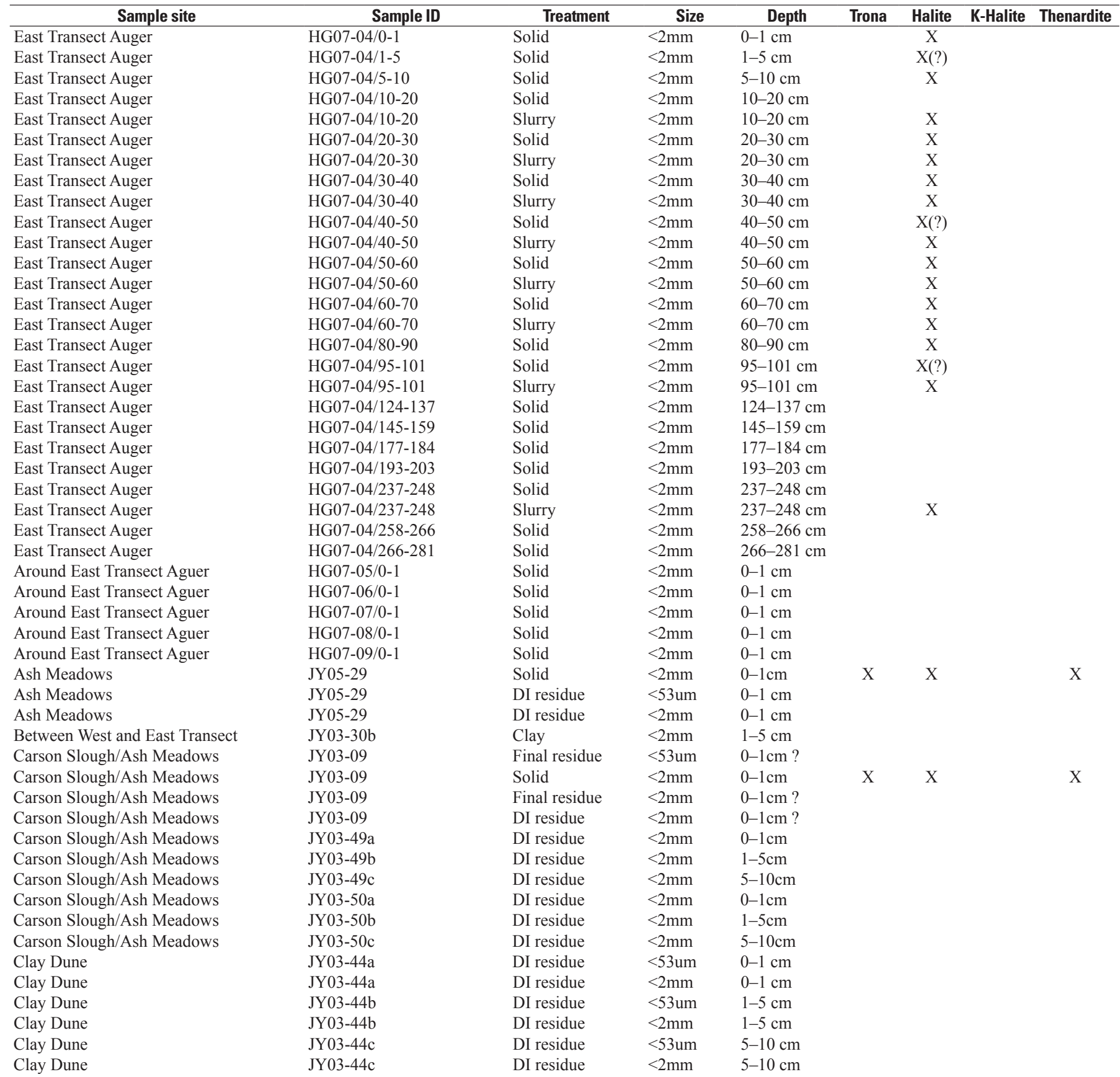


Table 15. Mineralogical data of auger hole and grab samples determined by X-ray diffraction.-Continued

[Sample site, name of sampling site; Sample ID, unique sample identifier; Treatment, type of sample preparation; Size, size fraction analyzed; Solid, unaltered bulk material; Slurry, water and sediment mixture; DI residue, remaining material after DI leaching; Clay, clay separates; Final residue, remaining material after nitric acid leaching; X, mineral is detected; I, illite; K, kaolinite; Se, sepiolite; Chl, chlorite; S, smectite/montmorillonite/beidellite; C, clinoptilolite; H, heulandite; M, mordentie; A, analcime; Z, unknown zeolite; P, plagioclase; K-spar, potassium feldspar; Y, yeelimite; Aph, aphthitalite; G, gaylussite; Ar, aragonite; $\mathrm{Mg}$, magnesite; Th, thermonatrite; $\mathrm{N}$, norsethite]

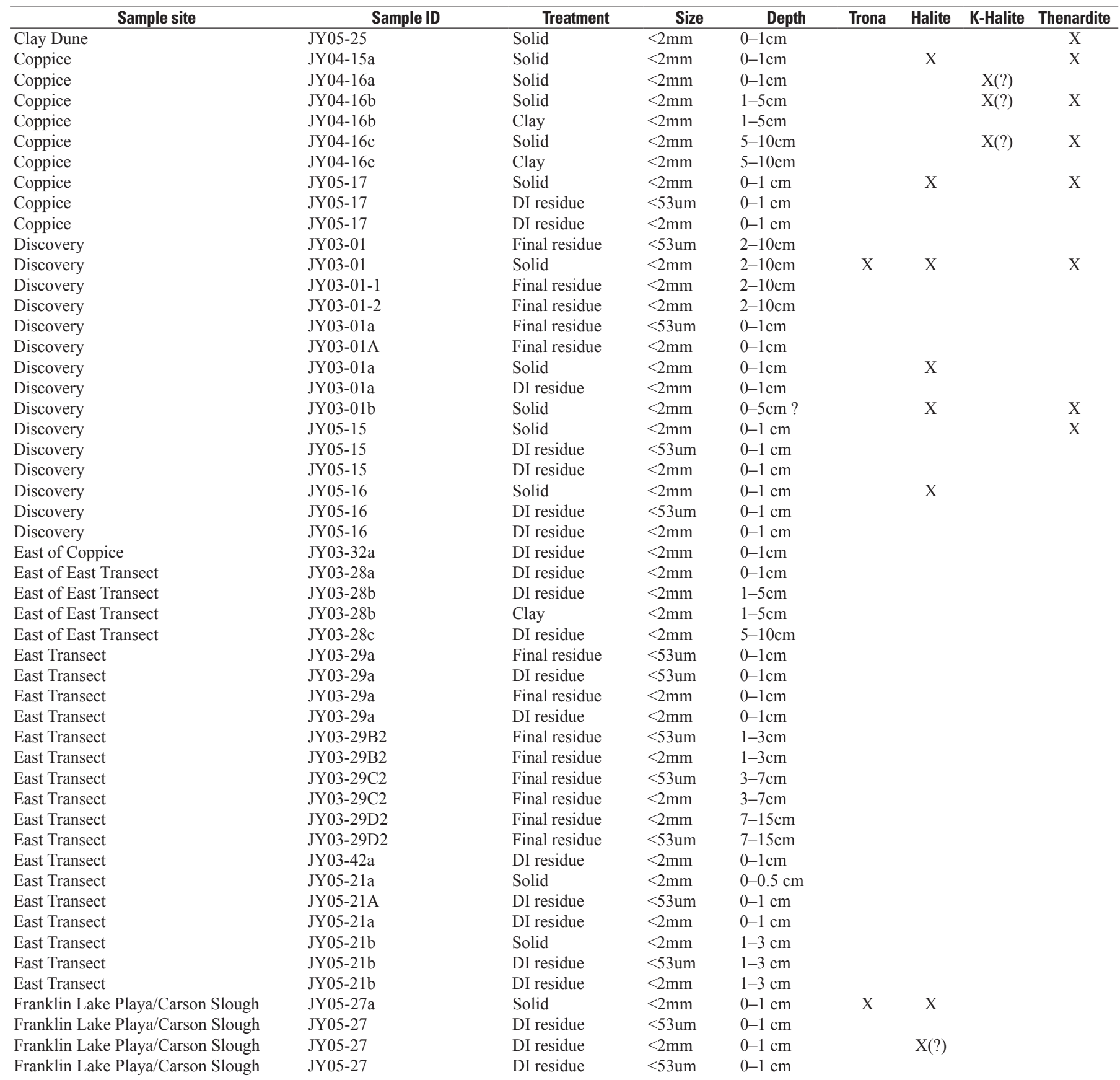




\section{Table 15. Mineralogical data of auger hole and grab samples determined by X-ray diffraction.-Continued}

[Sample site, name of sampling site; Sample ID, unique sample identifier; Treatment, type of sample preparation; Size, size fraction analyzed; Solid, unaltered bulk material; Slurry, water and sediment mixture; DI residue, remaining material after DI leaching; Clay, clay separates; Final residue, remaining material after nitric acid leaching; X, mineral is detected; I, illite; K, kaolinite; Se, sepiolite; Chl, chlorite; S, smectite/montmorillonite/beidellite; C, clinoptilolite; H, heulandite; M, mordentie; A, analcime; Z, unknown zeolite; P, plagioclase; K-spar, potassium feldspar; Y, yeelimite; Aph, aphthitalite; G, gaylussite; Ar, aragonite; $\mathrm{Mg}$, magnesite; Th, thermonatrite; $\mathrm{N}$, norsethite]

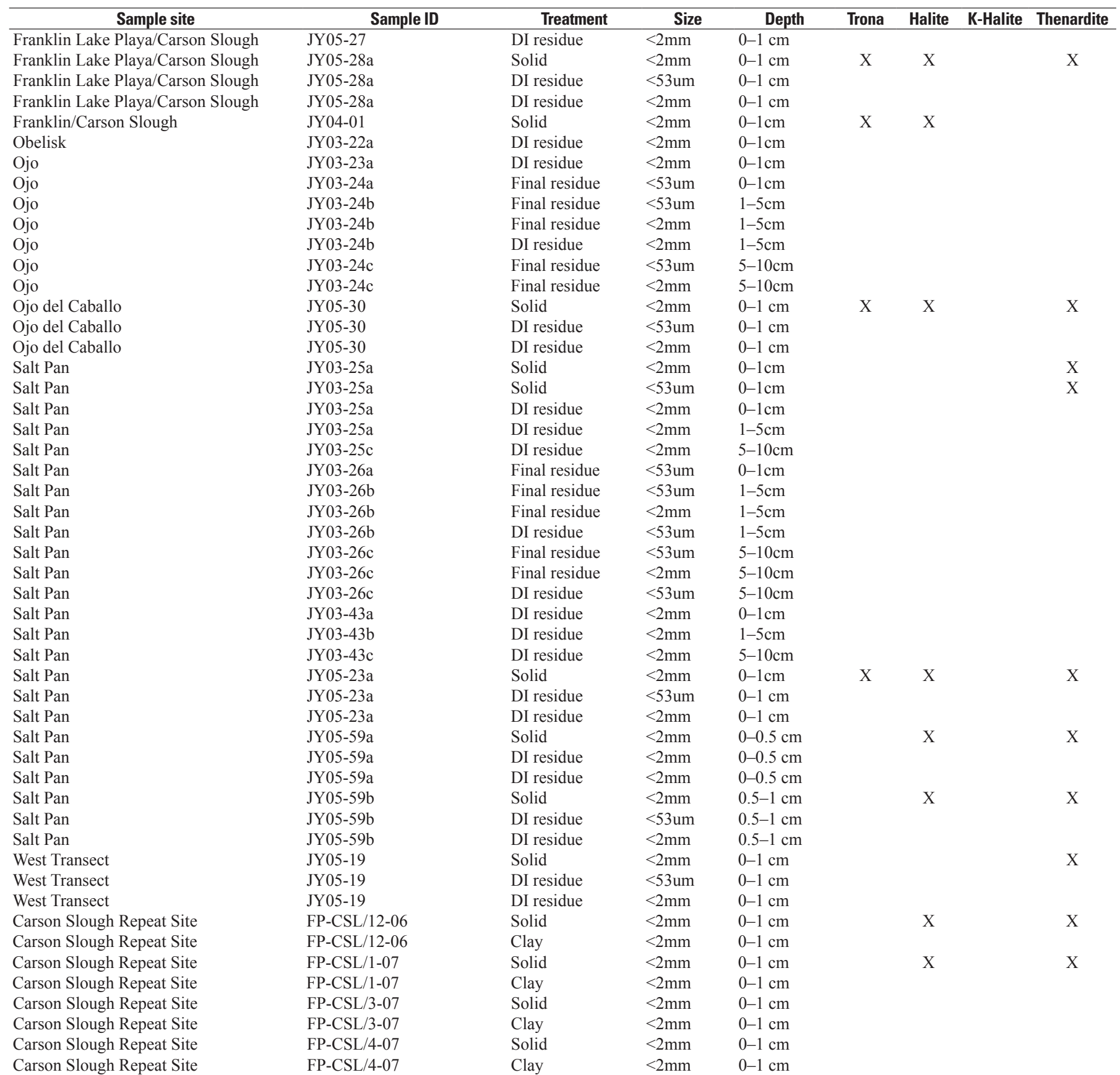


Table 15. Mineralogical data of auger hole and grab samples determined by X-ray diffraction.-Continued

[Sample site, name of sampling site; Sample ID, unique sample identifier; Treatment, type of sample preparation; Size, size fraction analyzed; Solid, unaltered bulk material; Slurry, water and sediment mixture; DI residue, remaining material after DI leaching; Clay, clay separates; Final residue, remaining material after nitric acid leaching; X, mineral is detected; I, illite; K, kaolinite; Se, sepiolite; Chl, chlorite; S, smectite/montmorillonite/beidellite; C, clinoptilolite; H, heulandite; M, mordentie; A, analcime; Z, unknown zeolite; P, plagioclase; K-spar, potassium feldspar; Y, yeelimite; Aph, aphthitalite; G, gaylussite; Ar, aragonite; $\mathrm{Mg}$, magnesite; Th, thermonatrite; $\mathrm{N}$, norsethite]

\begin{tabular}{|c|c|c|c|c|c|c|c|c|}
\hline Sample site & Sample ID & Treatment & Size & Depth & Trona & Halite & K-Halite & Thenardite \\
\hline Carson Slough Repeat Site & FP-CSL/7-07 & Solid & $<2 \mathrm{~mm}$ & $0-1 \mathrm{~cm}$ & & $\mathrm{X}$ & & $\mathrm{X}$ \\
\hline Carson Slough Repeat Site & FP-CSL/7-07 & Clay & $<2 \mathrm{~mm}$ & $0-1 \mathrm{~cm}$ & & & & \\
\hline Carson Slough Repeat Site & FP-CSL/10-07 & Clay & $<2 \mathrm{~mm}$ & $0-1 \mathrm{~cm}$ & & & & \\
\hline Carson Slough Repeat Site & FP-CSL/11-07 & Solid & $<2 \mathrm{~mm}$ & $0-1 \mathrm{~cm}$ & & $\mathrm{X}$ & & $\mathrm{X}$ \\
\hline Carson Slough Repeat Site & FP-CSL/11-07 & Clay & $<2 \mathrm{~mm}$ & $0-1 \mathrm{~cm}$ & & & & \\
\hline Carson Slough Repeat Site & FP-CSL/11-08 & Solid & $<2 \mathrm{~mm}$ & $0-1 \mathrm{~cm}$ & $\mathrm{X}$ ? & $\mathrm{X}$ & & \\
\hline Carson Slough Repeat Site & FP-CSL/11-08 & Clay & $<2 \mathrm{~mm}$ & $0-1 \mathrm{~cm}$ & & & & \\
\hline Carson Slough Repeat Site & FP-CSL/1-09 & Solid & $<2 \mathrm{~mm}$ & $0-1 \mathrm{~cm}$ & $\mathrm{X}$ & $\mathrm{X}$ & & $\mathrm{X}$ \\
\hline Carson Slough Repeat Site & FP-CSL/1-09 & Clay & $<2 \mathrm{~mm}$ & $0-1 \mathrm{~cm}$ & & & & \\
\hline Clay Dune Repeat Site & FP-CDN/12-06 & Solid & $<2 \mathrm{~mm}$ & $0-1 \mathrm{~cm}$ & & $\mathrm{X}$ & & $\mathrm{X}$ \\
\hline Clay Dune Repeat Site & FP-CDN/12-06 & Clay & $<2 \mathrm{~mm}$ & $0-1 \mathrm{~cm}$ & & & & \\
\hline Clay Dune Repeat Site & FP-CDN/4-07 & Clay & $<2 \mathrm{~mm}$ & $0-1 \mathrm{~cm}$ & & & & \\
\hline Clay Dune Repeat Site & FP-CDN/5-07 & Solid & $<2 \mathrm{~mm}$ & $0-1 \mathrm{~cm}$ & & $\mathrm{X}$ & & $\mathrm{X}$ \\
\hline Clay Dune Repeat Site & FP-CDN/5-07 & Clay & $<2 \mathrm{~mm}$ & $0-1 \mathrm{~cm}$ & & & & \\
\hline Clay Dune Repeat Site & FP-CDN/7-07 & Solid & $<2 \mathrm{~mm}$ & $0-1 \mathrm{~cm}$ & & $\mathrm{X}$ & & $\mathrm{X}$ \\
\hline Clay Dune Repeat Site & FP-CDN/7-07 & Clay & $<2 \mathrm{~mm}$ & $0-1 \mathrm{~cm}$ & & & & \\
\hline Clay Dune Repeat Site & FP-CDN/10-07 & Solid & $<2 \mathrm{~mm}$ & $0-1 \mathrm{~cm}$ & & $\mathrm{X}$ & & \\
\hline Clay Dune Repeat Site & FP-CDN/10-07 & Clay & $<2 \mathrm{~mm}$ & $0-1 \mathrm{~cm}$ & & & & \\
\hline Clay Dune Repeat Site & FP-CDN/11-07 & Solid & $<2 \mathrm{~mm}$ & $0-1 \mathrm{~cm}$ & & $\mathrm{X}$ & & \\
\hline Clay Dune Repeat Site & FP-CDN/11-07 & Clay & $<2 \mathrm{~mm}$ & $0-1 \mathrm{~cm}$ & & & & \\
\hline Clay Dune Repeat Site & FP-CDN/4-08 & Solid & $<2 \mathrm{~mm}$ & $0-1 \mathrm{~cm}$ & $\mathrm{X}$ & $\mathrm{X}$ & & \\
\hline Clay Dune Repeat Site & FP-CDN/4-08 & Clay & $<2 \mathrm{~mm}$ & $0-1 \mathrm{~cm}$ & & & & \\
\hline Clay Dune Repeat Site & FP-CDN/11-08 & Solid & $<2 \mathrm{~mm}$ & $0-1 \mathrm{~cm}$ & $\mathrm{X}$ & & & \\
\hline Clay Dune Repeat Site & FP-CDN/11-08 & Clay & $<2 \mathrm{~mm}$ & $0-1 \mathrm{~cm}$ & & & & \\
\hline Discovery Repeat Site & FP-DSC/4-07 & Solid & $<2 \mathrm{~mm}$ & $0-1 \mathrm{~cm}$ & & $\mathrm{X}$ & & $\mathrm{X}$ \\
\hline Discovery Repeat Site & FP-DSC/4-07 & Clay & $<2 \mathrm{~mm}$ & $0-1 \mathrm{~cm}$ & & & & \\
\hline Discovery Repeat Site & FP-DSC/5-07 & Solid & $<2 \mathrm{~mm}$ & $0-1 \mathrm{~cm}$ & & $\mathrm{X}$ & & \\
\hline Discovery Repeat Site & FP-DSC/5-07 & Clay & $<2 \mathrm{~mm}$ & $0-1 \mathrm{~cm}$ & & & & \\
\hline Discovery Repeat Site & FP-DSC/7-07 & Solid & $<2 \mathrm{~mm}$ & $0-1 \mathrm{~cm}$ & & $\mathrm{X}$ & & $\mathrm{X}$ \\
\hline Discovery Repeat Site & FP-DSC/7-07 & Clay & $<2 \mathrm{~mm}$ & $0-1 \mathrm{~cm}$ & & & & \\
\hline Discovery Repeat Site & FP-DSC/10-07 & Solid & $<2 \mathrm{~mm}$ & $0-1 \mathrm{~cm}$ & & $\mathrm{X}$ & & $X(?)$ \\
\hline Discovery Repeat Site & FP-DSC/10-07 & Clay & $<2 \mathrm{~mm}$ & $0-1 \mathrm{~cm}$ & & & & \\
\hline Discovery Repeat Site & FP-DSC/11-07 & Solid & $<2 \mathrm{~mm}$ & $0-1 \mathrm{~cm}$ & & $\mathrm{X}$ & & $\mathrm{X}$ \\
\hline
\end{tabular}


Table 15. Mineralogical data of auger hole and grab samples determined by X-ray diffraction.-Continued

[Sample site, name of sampling site; Sample ID, unique sample identifier; Treatment, type of sample preparation; Size, size fraction analyzed; Solid, unaltered bulk material; Slurry, water and sediment mixture; DI residue, remaining material after DI leaching; Clay, clay separates; Final residue, remaining material after nitric acid leaching; X, mineral is detected; I, illite; K, kaolinite; Se, sepiolite; Chl, chlorite; S, smectite/montmorillonite/beidellite; C, clinoptilolite; $\mathrm{H}$, heulandite; M, mordentie; A, analcime; Z, unknown zeolite; P, plagioclase; K-spar, potassium feldspar; Y, yeelimite; Aph, aphthitalite; G, gaylussite; Ar, aragonite; $\mathrm{Mg}$, magnesite; $\mathrm{Th}$, thermonatrite; $\mathrm{N}$, norsethite]

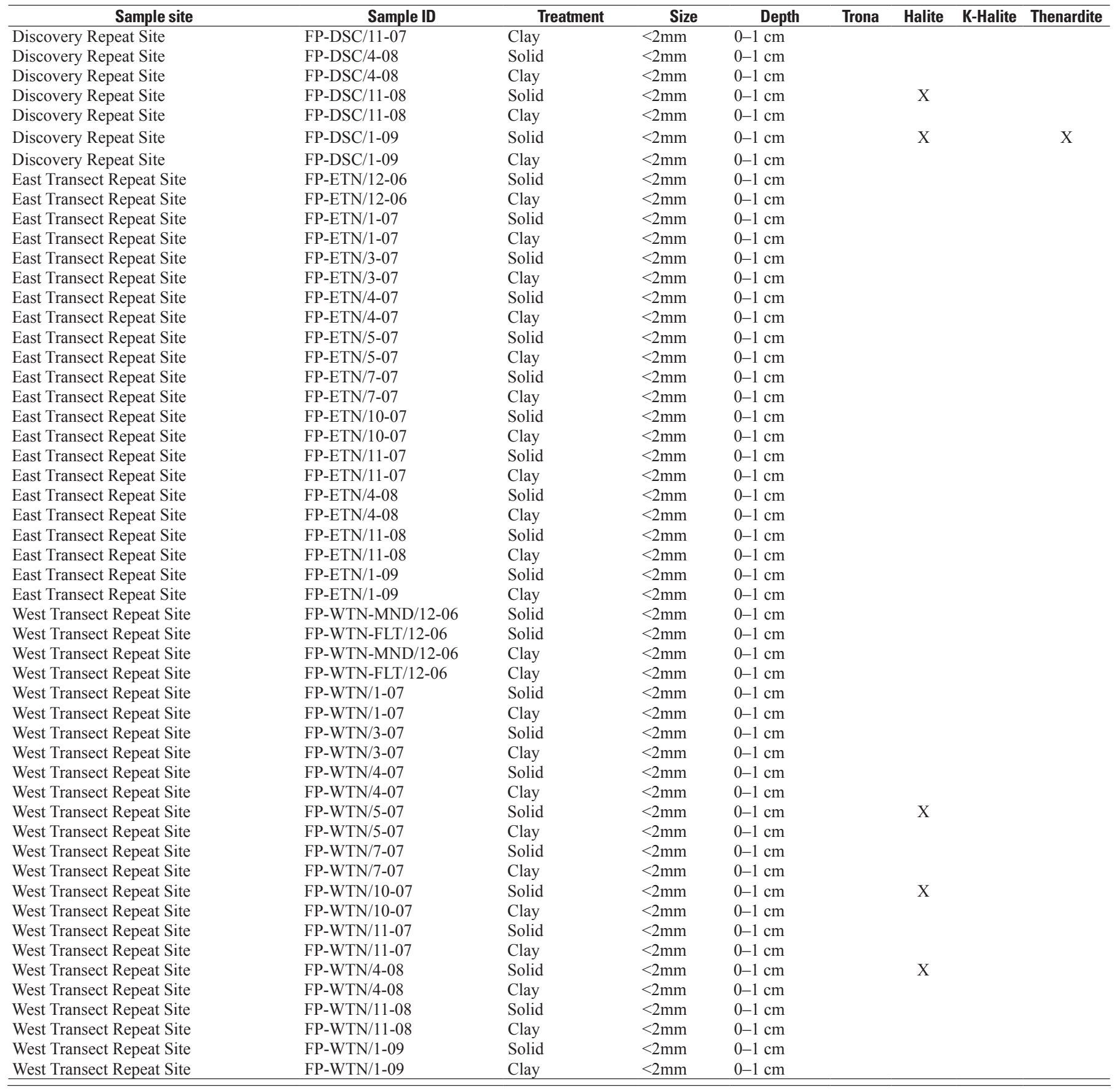


Table 15. Mineralogical data of auger hole and grab samples determined by X-ray diffraction.-Continued

[Sample site, name of sampling site; Sample ID, unique sample identifier; Treatment, type of sample preparation; Size, size fraction analyzed; Solid, unaltered bulk material; Slurry, water and sediment mixture; DI residue, remaining material after DI leaching; Clay, clay separates; Final residue, remaining material after nitric acid leaching; X, mineral is detected; I, illite; K, kaolinite; Se, sepiolite; Chl, chlorite; S, smectite/montmorillonite/beidellite; C, clinoptilolite; $\mathrm{H}$, heulandite; M, mordentie; A, analcime; Z, unknown zeolite; P, plagioclase; K-spar, potassium feldspar; Y, yeelimite; Aph, aphthitalite; G, gaylussite; Ar, aragonite; $\mathrm{Mg}$, magnesite; Th, thermonatrite; $\mathrm{N}$, norsethite]

\begin{tabular}{|c|c|c|c|c|c|c|c|c|}
\hline Sample site & Sample ID & Burkeite & Calcite & Dolomite & Clay & Amph. & Zeolite & Andalusite \\
\hline East Transect Auger & HG07-04/0-1 & & $\mathrm{X}$ & & I & & A & \\
\hline East Transect Auger & HG07-04/1-5 & & $\mathrm{X}$ & $\mathrm{X}$ & I & & A & \\
\hline East Transect Auger & HG07-04/5-10 & & $\mathrm{X}$ & & I & & A & \\
\hline East Transect Auger & HG07-04/10-20 & & $\mathrm{X}$ & & I & & A & \\
\hline East Transect Auger & HG07-04/10-20 & & $\mathrm{X}$ & & $\mathrm{I}, \mathrm{K}$ & & A & \\
\hline East Transect Auger & HG07-04/20-30 & & $\mathrm{X}$ & $\mathrm{X}$ & I & & A & \\
\hline East Transect Auger & HG07-04/20-30 & & $\mathrm{X}$ & & $\mathrm{I}, \mathrm{K}$ & & A & \\
\hline East Transect Auger & HG07-04/30-40 & & $\mathrm{X}$ & & I & & A & \\
\hline East Transect Auger & HG07-04/30-40 & & $\mathrm{X}$ & & I & & A & \\
\hline East Transect Auger & HG07-04/40-50 & & $\mathrm{X}$ & $X(?)$ & & & A & \\
\hline East Transect Auger & HG07-04/40-50 & & $\mathrm{X}$ & & $\mathrm{I}, \mathrm{S}$ & & & \\
\hline East Transect Auger & HG07-04/50-60 & & $\mathrm{X}$ & & I & & A & \\
\hline East Transect Auger & HG07-04/50-60 & & $\mathrm{X}$ & & I & & A & \\
\hline East Transect Auger & HG07-04/60-70 & & $\mathrm{X}$ & & & & A & \\
\hline East Transect Auger & HG07-04/60-70 & & $\mathrm{X}$ & & I & & A & \\
\hline East Transect Auger & HG07-04/80-90 & & $\mathrm{X}$ & $\mathrm{X}$ & I & & A & \\
\hline East Transect Auger & HG07-04/95-101 & & $\mathrm{X}$ & $\mathrm{X}(?)$ & & & $\mathrm{A}(?)$ & \\
\hline East Transect Auger & HG07-04/95-101 & & $\mathrm{X}$ & & $\mathrm{I}, \mathrm{K}, \mathrm{S}$ & & A & \\
\hline East Transect Auger & HG07-04/124-137 & & $\mathrm{X}$ & & $\mathrm{I}(?)$ & & & \\
\hline East Transect Auger & HG07-04/145-159 & & $\mathrm{X}$ & $X(?)$ & & & $\mathrm{A}(?)$ & \\
\hline East Transect Auger & HG07-04/177-184 & & $\mathrm{X}$ & & & & & \\
\hline East Transect Auger & HG07-04/193-203 & & $\mathrm{X}$ & & & & & \\
\hline East Transect Auger & HG07-04/237-248 & & $\mathrm{X}$ & & & & & \\
\hline East Transect Auger & HG07-04/237-248 & & $\mathrm{X}$ & & & & & \\
\hline East Transect Auger & HG07-04/258-266 & & $\mathrm{X}$ & & & & & \\
\hline East Transect Auger & HG07-04/266-281 & & $\mathrm{X}$ & & & & & \\
\hline Around East Transect Aguer & HG07-05/0-1 & & $\mathrm{X}$ & $\mathrm{X}(?)$ & & & & \\
\hline Around East Transect Aguer & HG07-06/0-1 & & $\mathrm{X}$ & & & & & \\
\hline Around East Transect Aguer & HG07-07/0-1 & & $\mathrm{X}$ & $\mathrm{X}$ & & & & \\
\hline Around East Transect Aguer & HG07-08/0-1 & & $\mathrm{X}$ & & & & A & \\
\hline Around East Transect Aguer & HG07-09/0-1 & & $\mathrm{X}$ & $\mathrm{X}$ & I & & A & \\
\hline Ash Meadows & JY05-29 & $\mathrm{X}$ & $\mathrm{X}$ & & I & & & \\
\hline Ash Meadows & JY05-29 & & $\mathrm{X}$ & $\mathrm{X}$ & $\mathrm{I}, \mathrm{K}$ & & & \\
\hline Ash Meadows & JY05-29 & & $\mathrm{X}$ & $\mathrm{X}$ & I & $\mathrm{X}$ & & \\
\hline Between West and East Transect & JY03-30b & & & & & & A & \\
\hline Carson Slough/Ash Meadows & JY03-09 & & $\mathrm{X}$ & $\mathrm{X}$ & I & & & \\
\hline Carson Slough/Ash Meadows & JY03-09 & $\mathrm{X}$ & $\mathrm{X}$ & $\mathrm{X}$ & & & & \\
\hline Carson Slough/Ash Meadows & JY03-09 & & $\mathrm{X}$ & & I & & & \\
\hline Carson Slough/Ash Meadows & JY03-09 & & $\mathrm{X}$ & & $\mathrm{I}, \mathrm{K}$ & $\mathrm{X}$ & & \\
\hline Carson Slough/Ash Meadows & JY03-49a & & $\mathrm{X}$ & $\mathrm{X}$ & $\mathrm{I}, \mathrm{K}$ & $\mathrm{X}$ & A & \\
\hline Carson Slough/Ash Meadows & JY03-49b & & $\mathrm{X}$ & $\mathrm{X}$ & I & & & \\
\hline Carson Slough/Ash Meadows & JY03-49c & & $\mathrm{X}$ & $\mathrm{X}$ & I & & & \\
\hline Carson Slough/Ash Meadows & JY03-50a & & $\mathrm{X}$ & $\mathrm{X}$ & I & & A (?) & \\
\hline Carson Slough/Ash Meadows & JY03-50b & & $\mathrm{X}$ & $\mathrm{X}$ & I & & & \\
\hline Carson Slough/Ash Meadows & JY03-50c & & $\mathrm{X}$ & $\mathrm{X}$ & & & & \\
\hline Clay Dune & JY03-44a & & $\mathrm{X}$ & & $\mathrm{I}, \mathrm{K}$ & $\mathrm{X}$ & A & \\
\hline Clay Dune & JY03-44a & & $\mathrm{X}$ & & $\mathrm{I}, \mathrm{K}$ & & & $\mathrm{X}(?)$ \\
\hline Clay Dune & JY03-44b & & $\mathrm{X}$ & & $\mathrm{I}, \mathrm{K}$ & $\mathrm{X}$ & A & \\
\hline Clay Dune & JY03-44b & & $\mathrm{X}$ & & $\mathrm{I}, \mathrm{K}$ & & A & \\
\hline Clay Dune & JY03-44c & & $\mathrm{X}$ & & $\mathrm{I}, \mathrm{K}$ & $\mathrm{X}$ & A & \\
\hline Clay Dune & JY03-44c & & $\mathrm{X}$ & & $\mathrm{I}, \mathrm{K}$ & & A & \\
\hline Clay Dune & JY05-25 & & $\mathrm{X}$ & & $\mathrm{I}, \mathrm{K}$ & & A & \\
\hline Coppice & JY04-15a & & $\mathrm{X}$ & & I & & & \\
\hline
\end{tabular}


Table 15. Mineralogical data of auger hole and grab samples determined by X-ray diffraction._-Continued

[Sample site, name of sampling site; Sample ID, unique sample identifier; Treatment, type of sample preparation; Size, size fraction analyzed; Solid, unaltered bulk material; Slurry, water and sediment mixture; DI residue, remaining material after DI leaching; Clay, clay separates; Final residue, remaining material after nitric acid leaching; X, mineral is detected; I, illite; K, kaolinite; Se, sepiolite; Chl, chlorite; S, smectite/montmorillonite/beidellite; C, clinoptilolite; H, heulandite; M, mordentie; A, analcime; Z, unknown zeolite; P, plagioclase; K-spar, potassium feldspar; Y, yeelimite; Aph, aphthitalite; G, gaylussite; Ar, aragonite; $\mathrm{Mg}$, magnesite; Th, thermonatrite; $\mathrm{N}$, norsethite]

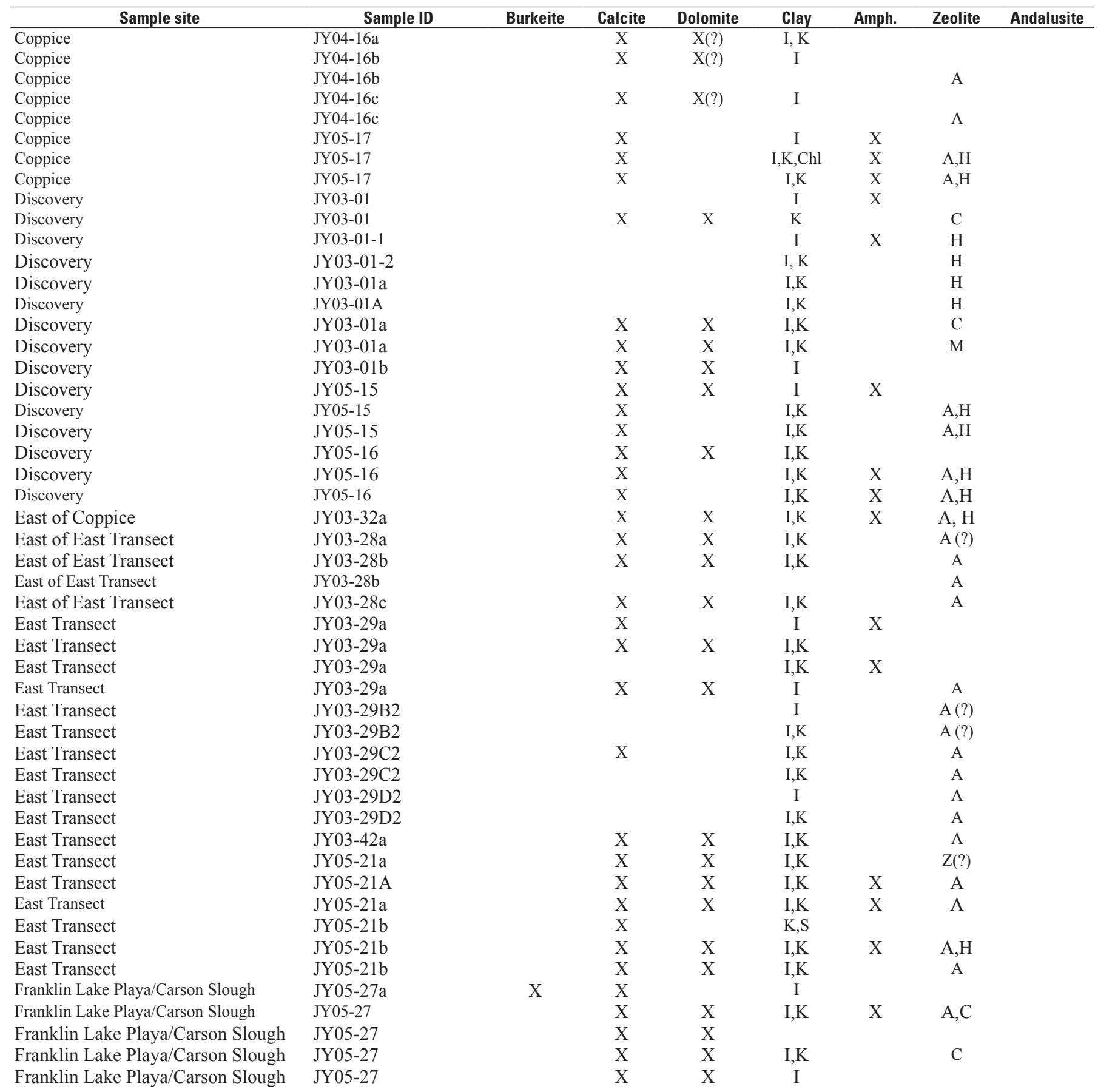


Table 15. Mineralogical data of auger hole and grab samples determined by X-ray diffraction._-Continued

[Sample site, name of sampling site; Sample ID, unique sample identifier; Treatment, type of sample preparation; Size, size fraction analyzed; Solid, unaltered bulk material; Slurry, water and sediment mixture; DI residue, remaining material after DI leaching; Clay, clay separates; Final residue, remaining material after nitric acid leaching; X, mineral is detected; I, illite; K, kaolinite; Se, sepiolite; Chl, chlorite; S, smectite/montmorillonite/beidellite; C, clinoptilolite; H, heulandite; M, mordentie; A, analcime; Z, unknown zeolite; P, plagioclase; K-spar, potassium feldspar; Y, yeelimite; Aph, aphthitalite; G, gaylussite; Ar, aragonite; Mg, magnesite; Th, thermonatrite; $\mathrm{N}$, norsethite]

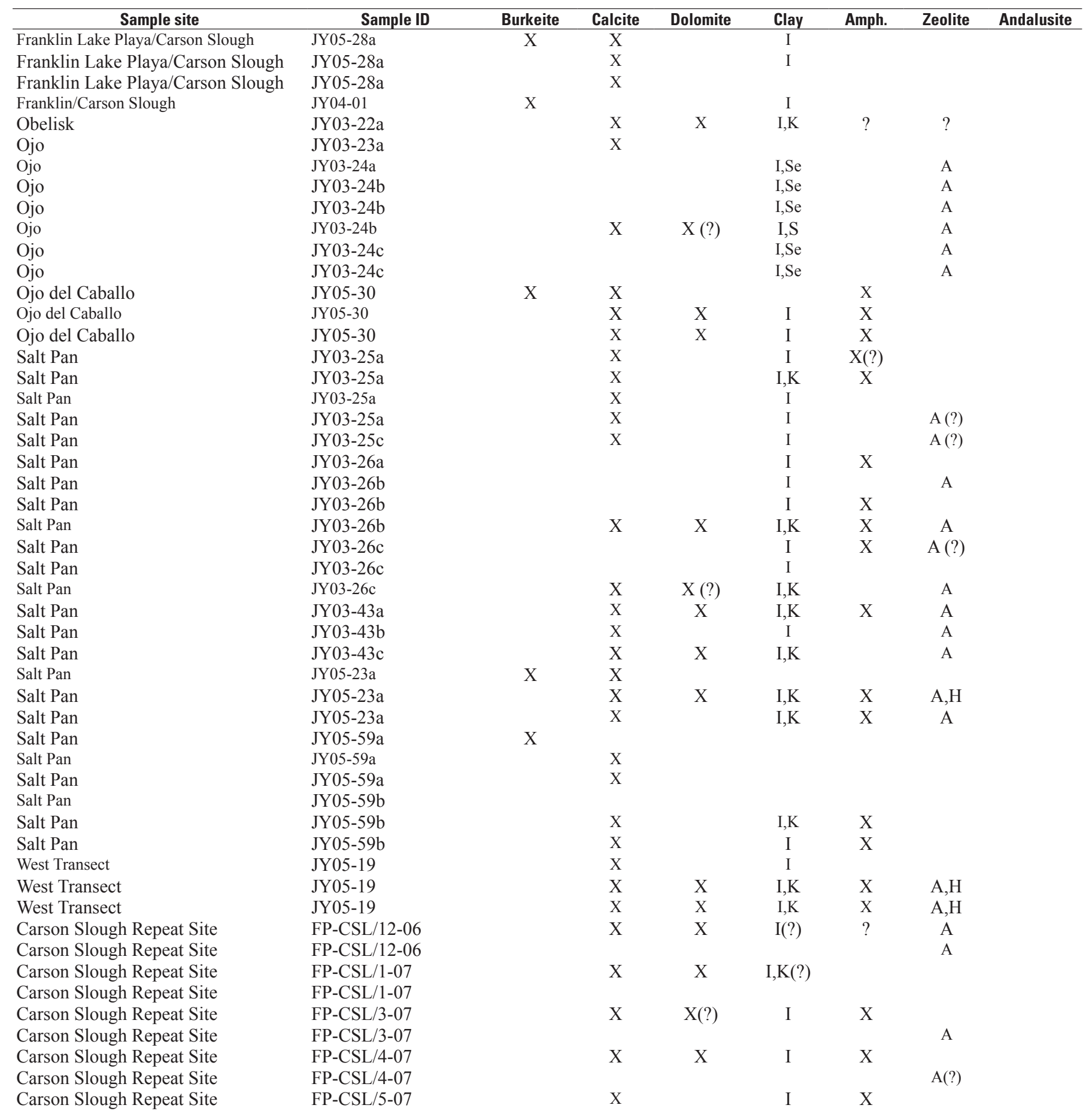


Table 15. Mineralogical data of auger hole and grab samples determined by X-ray diffraction.-Continued

[Sample site, name of sampling site; Sample ID, unique sample identifier; Treatment, type of sample preparation; Size, size fraction analyzed; Solid, unaltered bulk material; Slurry, water and sediment mixture; DI residue, remaining material after DI leaching; Clay, clay separates; Final residue, remaining material after nitric acid leaching; X, mineral is detected; I, illite; K, kaolinite; Se, sepiolite; Chl, chlorite; S, smectite/montmorillonite/beidellite; C, clinoptilolite; $\mathrm{H}$, heulandite; M, mordentie; A, analcime; Z, unknown zeolite; P, plagioclase; K-spar, potassium feldspar; Y, yeelimite; Aph, aphthitalite; G, gaylussite; Ar, aragonite; $\mathrm{Mg}$, magnesite; Th, thermonatrite; $\mathrm{N}$, norsethite]

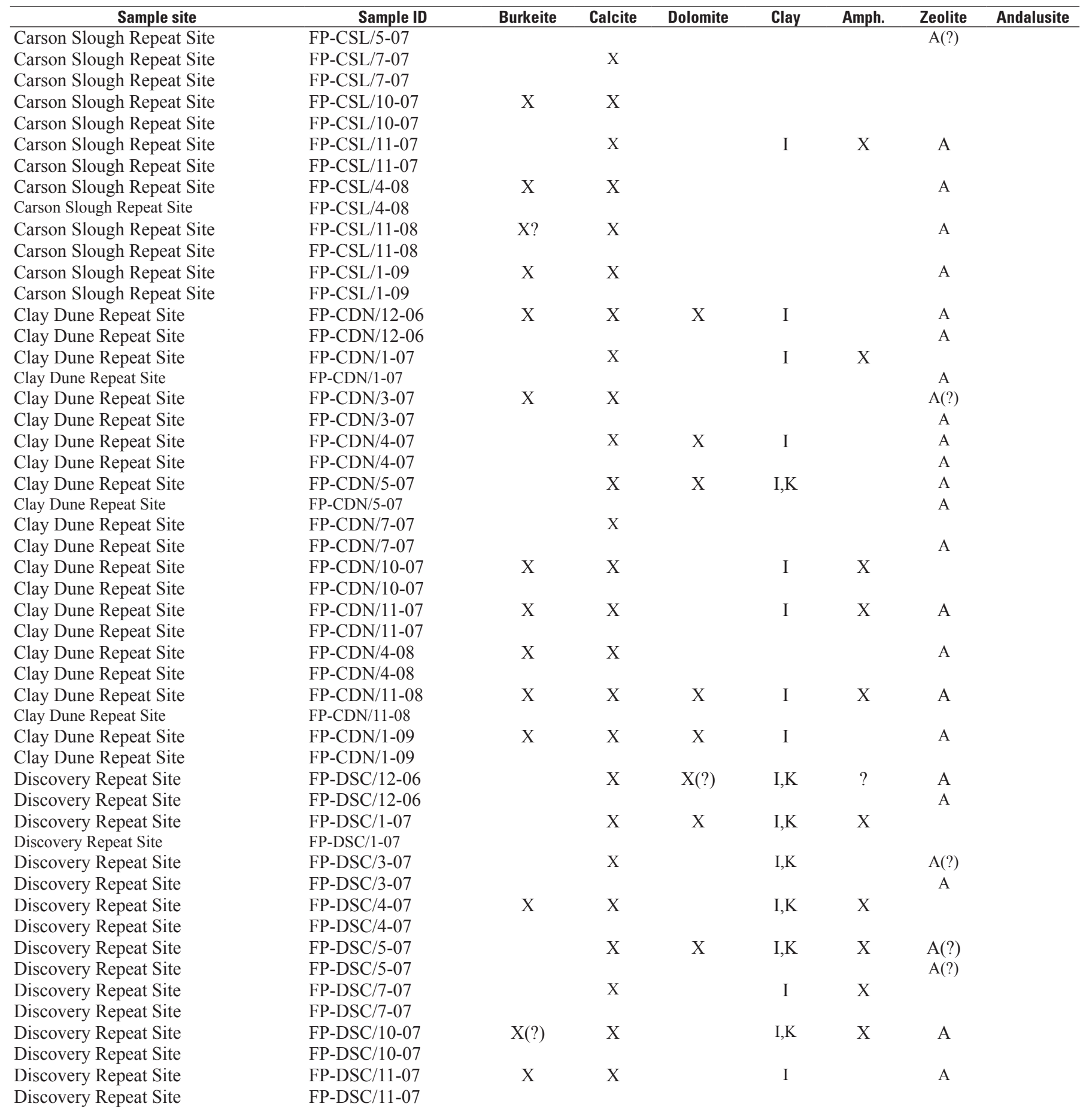


Table 15. Mineralogical data of auger hole and grab samples determined by X-ray diffraction.-Continued

[Sample site, name of sampling site; Sample ID, unique sample identifier; Treatment, type of sample preparation; Size, size fraction analyzed; Solid, unaltered bulk material; Slurry, water and sediment mixture; DI residue, remaining material after DI leaching; Clay, clay separates; Final residue, remaining material after nitric acid leaching; X, mineral is detected; I, illite; K, kaolinite; Se, sepiolite; Chl, chlorite; S, smectite/montmorillonite/beidellite; C, clinoptilolite; $\mathrm{H}$, heulandite; M, mordentie; A, analcime; Z, unknown zeolite; P, plagioclase; K-spar, potassium feldspar; Y, yeelimite; Aph, aphthitalite; G, gaylussite; Ar, aragonite; $\mathrm{Mg}$, magnesite; Th, thermonatrite; $\mathrm{N}$, norsethite]

\begin{tabular}{|c|c|c|c|c|c|c|c|c|}
\hline Sample site & Sample ID & Burkeite & Calcite & Dolomite & Clay & Amph. & Zeolite & Andalusite \\
\hline Discovery Repeat Site & FP-DSC/4-08 & & $\mathrm{X}$ & $\mathrm{X}$ & $\mathrm{I}, \mathrm{K}$ & & A & \\
\hline Discovery Repeat Site & FP-DSC/4-08 & & & & & & & \\
\hline Discovery Repeat Site & FP-DSC/11-08 & $\mathrm{X} ?$ & $\mathrm{X}$ & $X$ & I & & A & \\
\hline Discovery Repeat Site & FP-DSC/11-08 & & & & & & & \\
\hline Discovery Repeat Site & FP-DSC/1-09 & & $\mathrm{X}$ & & $\mathrm{I}, \mathrm{K}$ & & A & \\
\hline Discovery Repeat Site & FP-DSC/1-09 & & & & & & & \\
\hline East Transect Repeat Site & FP-ETN/12-06 & & $\mathrm{X}$ & $\mathrm{X}$ & $\mathrm{I}, \mathrm{K}$ & & & \\
\hline East Transect Repeat Site & FP-ETN/12-06 & & & & & & $\mathrm{A}$ & \\
\hline East Transect Repeat Site & FP-ETN/1-07 & & $\mathrm{X}$ & $\mathrm{X}$ & $\mathrm{I}, \mathrm{K}(?)$ & & A & \\
\hline East Transect Repeat Site & FP-ETN/1-07 & & & & & & A & \\
\hline East Transect Repeat Site & FP-ETN/3-07 & & $\mathrm{X}$ & $\mathrm{X}(?)$ & $\mathrm{I}, \mathrm{K}$ & $X$ & A & \\
\hline East Transect Repeat Site & FP-ETN/3-07 & & & & & & $\mathrm{A}$ & \\
\hline East Transect Repeat Site & FP-ETN/4-07 & & $\mathrm{X}$ & $\mathrm{X}$ & I & & A & \\
\hline East Transect Repeat Site & FP-ETN/4-07 & & & & & & A & \\
\hline East Transect Repeat Site & FP-ETN/5-07 & & $\mathrm{X}$ & $\mathrm{X}$ & $\mathrm{I}, \mathrm{K}$ & & A & \\
\hline East Transect Repeat Site & FP-ETN/5-07 & & & & & & A & \\
\hline East Transect Repeat Site & FP-ETN/7-07 & & $\mathrm{X}$ & & I & & A & \\
\hline East Transect Repeat Site & FP-ETN/7-07 & & & & & & A & \\
\hline East Transect Repeat Site & FP-ETN/10-07 & & $\mathrm{X}$ & & I & & A & \\
\hline East Transect Repeat Site & FP-ETN/10-07 & & & & & & A & \\
\hline East Transect Repeat Site & FP-ETN/11-07 & & $\mathrm{X}$ & & I & & $\mathrm{A}$ & \\
\hline East Transect Repeat Site & FP-ETN/11-07 & & & & & & & \\
\hline East Transect Repeat Site & FP-ETN/4-08 & & $\mathrm{X}$ & $X$ & $\mathrm{I}, \mathrm{K}$ & $\mathrm{X}$ & A & \\
\hline East Transect Repeat Site & FP-ETN/4-08 & & & & & & & \\
\hline East Transect Repeat Site & FP-ETN/11-08 & & $\mathrm{X}$ & $\mathrm{X}$ & I & & A & \\
\hline East Transect Repeat Site & FP-ETN/11-08 & & & & & & & \\
\hline East Transect Repeat Site & FP-ETN/1-09 & & $\mathrm{X}$ & $X$ & $\mathrm{I}, \mathrm{K}$ & & A & \\
\hline East Transect Repeat Site & FP-ETN/1-09 & & & & & & & \\
\hline West Transect Repeat Site & FP-WTN-MND/12-06 & & $\mathrm{X}$ & $\mathrm{X}$ & $\mathrm{I}, \mathrm{K}$ & & $\mathrm{C}(?), \mathrm{A}(?)$ & \\
\hline West Transect Repeat Site & FP-WTN-FLT/12-06 & & $\mathrm{X}$ & $\mathrm{X}(?)$ & I & $\mathrm{X}(?)$ & $\mathrm{A}$ & \\
\hline West Transect Repeat Site & FP-WTN-MND/12-06 & & & & & & $\mathrm{A}(?)$ & \\
\hline West Transect Repeat Site & FP-WTN-FLT/12-06 & & & & & & $\mathrm{A}(?)$ & \\
\hline West Transect Repeat Site & FP-WTN/1-07 & & $\mathrm{X}$ & & $\mathrm{I}, \mathrm{K}(?)$ & $\mathrm{X}$ & & \\
\hline West Transect Repeat Site & FP-WTN/1-07 & & & & & & A & \\
\hline West Transect Repeat Site & FP-WTN/3-07 & & $\mathrm{X}$ & & I & & $\mathrm{A}$ & \\
\hline West Transect Repeat Site & FP-WTN/3-07 & & & & & & A & \\
\hline West Transect Repeat Site & FP-WTN/4-07 & & $\mathrm{X}$ & $X$ & $\mathrm{I}, \mathrm{K}$ & & A & \\
\hline West Transect Repeat Site & FP-WTN/4-07 & & & & & & $\mathrm{A}(?)$ & \\
\hline West Transect Repeat Site & FP-WTN/5-07 & & $\mathrm{X}$ & $\mathrm{X}(?)$ & $\mathrm{I}, \mathrm{K}$ & & A & \\
\hline West Transect Repeat Site & FP-WTN/5-07 & & & & & & $\mathrm{A}(?)$ & \\
\hline West Transect Repeat Site & FP-WTN/7-07 & & $\mathrm{X}$ & & I & $\mathrm{X}$ & & \\
\hline West Transect Repeat Site & FP-WTN/7-07 & & & & & & & \\
\hline West Transect Repeat Site & FP-WTN/10-07 & & $\mathrm{X}$ & & I & & & \\
\hline West Transect Repeat Site & FP-WTN/10-07 & & & & & & & \\
\hline West Transect Repeat Site & FP-WTN/11-07 & & $\mathrm{X}$ & & $\mathrm{I}, \mathrm{K}$ & & A & \\
\hline West Transect Repeat Site & FP-WTN/11-07 & & & & & & & \\
\hline West Transect Repeat Site & FP-WTN/4-08 & & $\mathrm{X}$ & & $\mathrm{I}, \mathrm{K}$ & & $\mathrm{A}$ & \\
\hline West Transect Repeat Site & FP-WTN/4-08 & & & & & & & \\
\hline West Transect Repeat Site & FP-WTN/11-08 & & $\mathrm{X}$ & $\mathrm{X}$ & I & & A & \\
\hline West Transect Repeat Site & FP-WTN/11-08 & & & & & & & \\
\hline West Transect Repeat Site & FP-WTN/1-09 & & $\mathrm{X}$ & & I & & $\mathrm{A}$ & \\
\hline West Transect Repeat Site & FP-WTN/1-09 & & & & & & & \\
\hline
\end{tabular}


Table 15. Mineralogical data of auger hole and grab samples determined by X-ray diffraction.-Continued

[Sample site, name of sampling site; Sample ID, unique sample identifier; Treatment, type of sample preparation; Size, size fraction analyzed; Solid, unaltered bulk material; Slurry, water and sediment mixture; DI residue, remaining material after DI leaching; Clay, clay separates; Final residue, remaining material after nitric acid leaching; X, mineral is detected; I, illite; K, kaolinite; Se, sepiolite; Chl, chlorite; S, smectite/montmorillonite/beidellite; C, clinoptilolite; $\mathrm{H}$, heulandite; M, mordentie; A, analcime; Z, unknown zeolite; P, plagioclase; K-spar, potassium feldspar; Y, yeelimite; Aph, aphthitalite; G, gaylussite; Ar, aragonite; Mg, magnesite; Th, thermonatrite; N, norsethite]

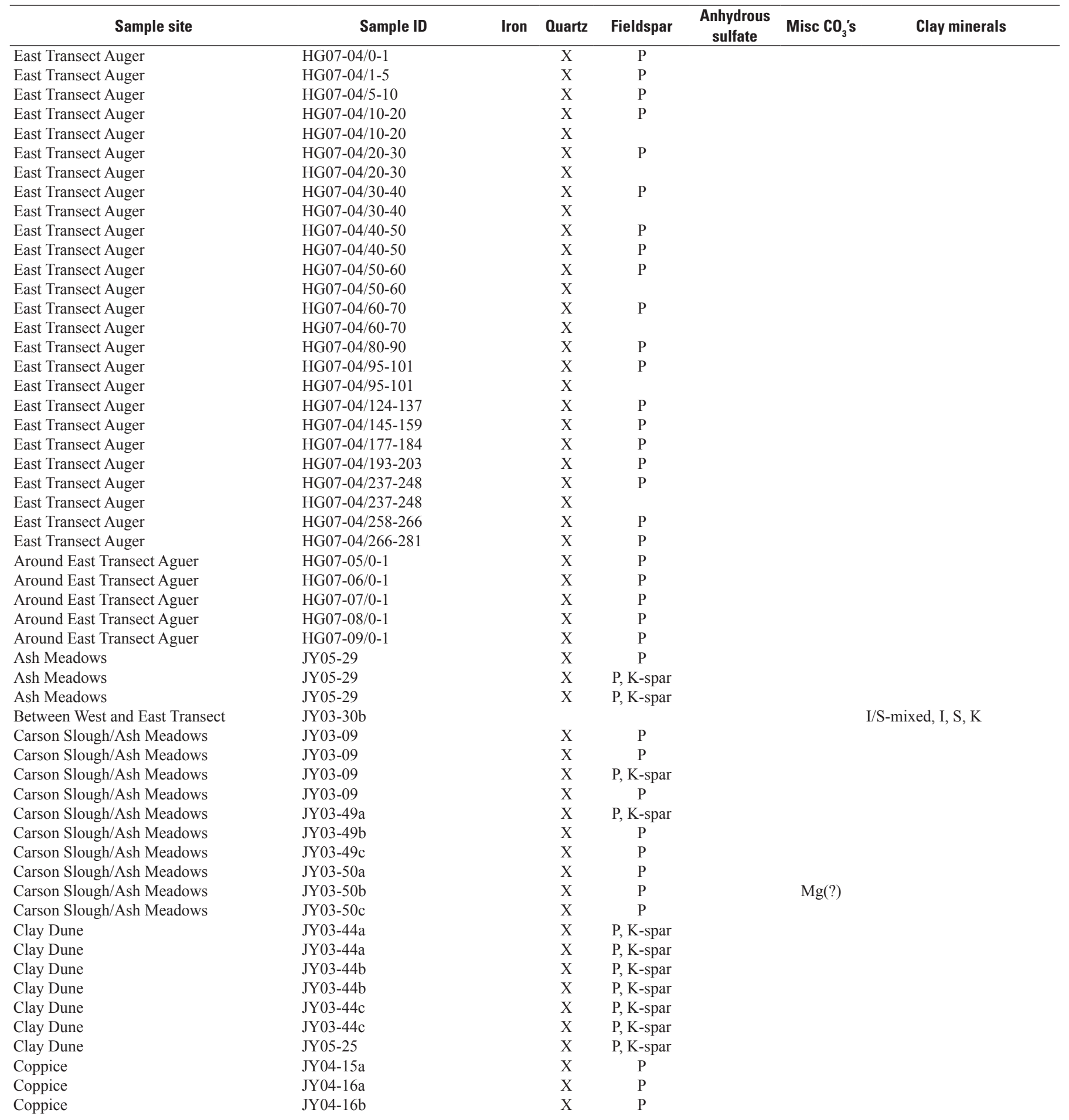


Table 15. Mineralogical data of auger hole and grab samples determined by X-ray diffraction._-Continued

[Sample site, name of sampling site; Sample ID, unique sample identifier; Treatment, type of sample preparation; Size, size fraction analyzed; Solid, unaltered bulk material; Slurry, water and sediment mixture; DI residue, remaining material after DI leaching; Clay, clay separates; Final residue, remaining material after nitric acid leaching; X, mineral is detected; I, illite; K, kaolinite; Se, sepiolite; Chl, chlorite; S, smectite/montmorillonite/beidellite; C, clinoptilolite; $\mathrm{H}$, heulandite; M, mordentie; A, analcime; Z, unknown zeolite; P, plagioclase; K-spar, potassium feldspar; Y, yeelimite; Aph, aphthitalite; G, gaylussite; Ar, aragonite; $\mathrm{Mg}$, magnesite; Th, thermonatrite; $\mathrm{N}$, norsethite]

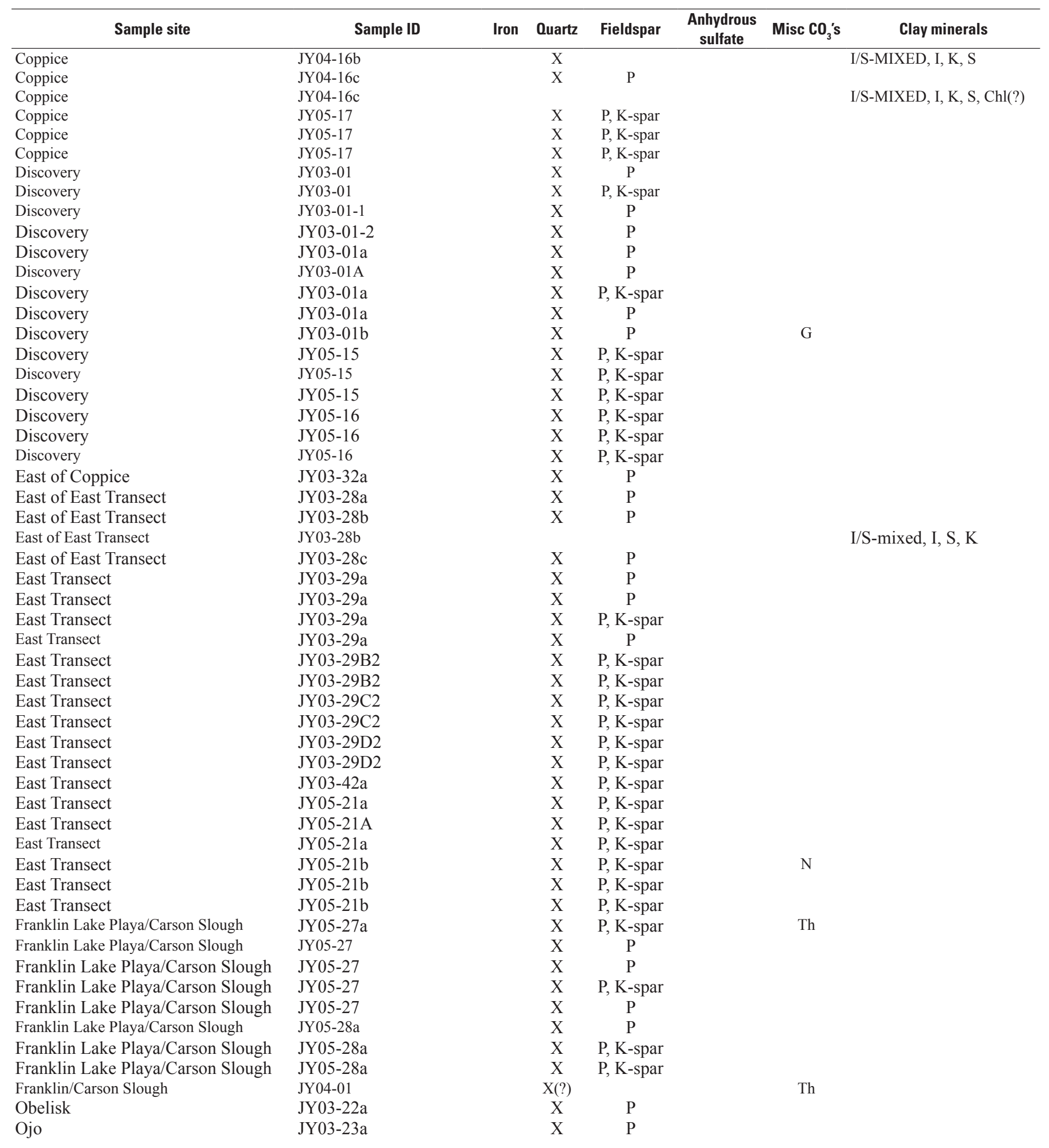


Table 15. Mineralogical data of auger hole and grab samples determined by X-ray diffraction.—Continued

[Sample site, name of sampling site; Sample ID, unique sample identifier; Treatment, type of sample preparation; Size, size fraction analyzed; Solid, unaltered bulk material; Slurry, water and sediment mixture; DI residue, remaining material after DI leaching; Clay, clay separates; Final residue, remaining material after nitric acid leaching; X, mineral is detected; I, illite; K, kaolinite; Se, sepiolite; Chl, chlorite; S, smectite/montmorillonite/beidellite; C, clinoptilolite; $\mathrm{H}$, heulandite; M, mordentie; A, analcime; Z, unknown zeolite; P, plagioclase; K-spar, potassium feldspar; Y, yeelimite; Aph, aphthitalite; G, gaylussite; Ar, aragonite; $\mathrm{Mg}$, magnesite; Th, thermonatrite; $\mathrm{N}$, norsethite]

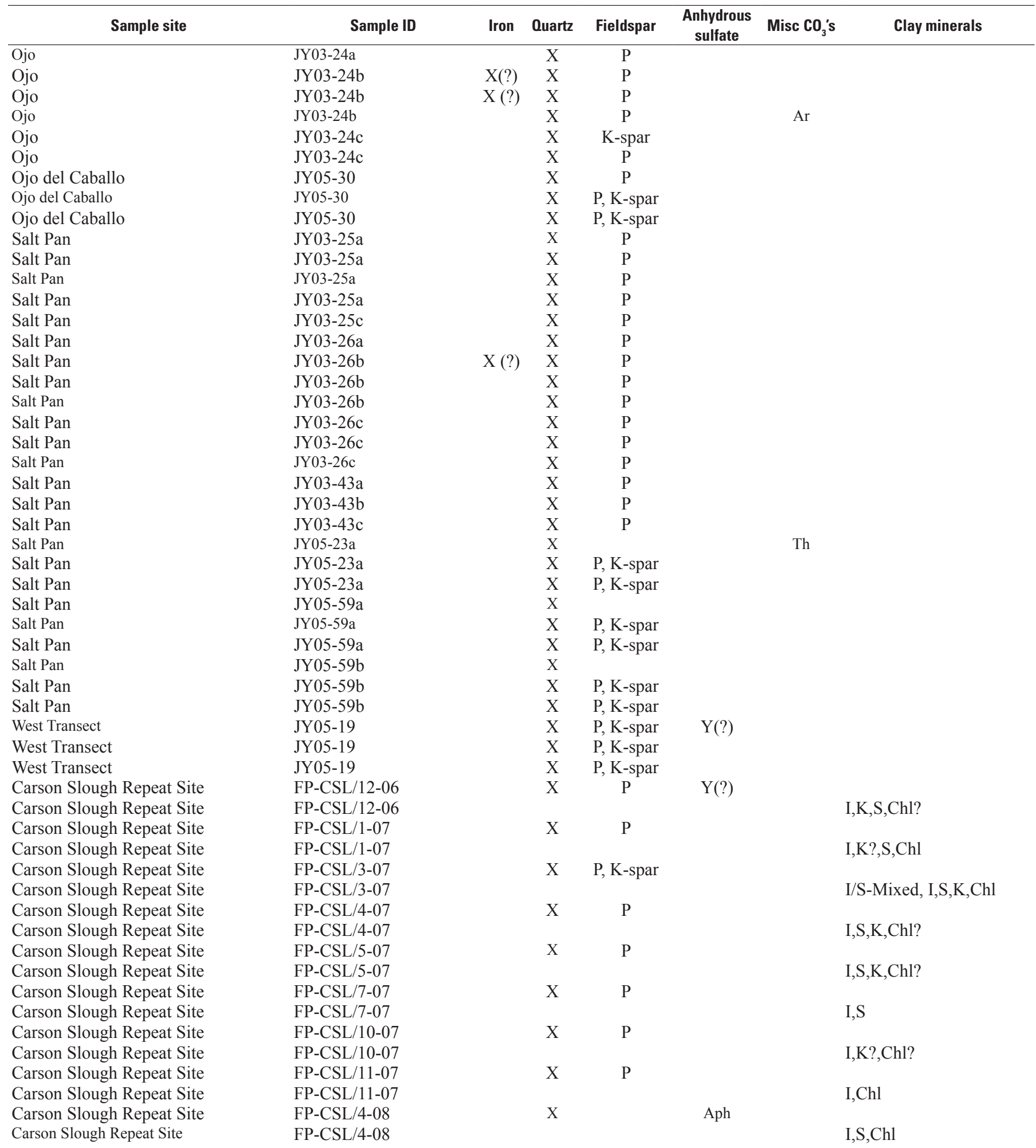


Table 15. Mineralogical data of auger hole and grab samples determined by X-ray diffraction.-Continued

[Sample site, name of sampling site; Sample ID, unique sample identifier; Treatment, type of sample preparation; Size, size fraction analyzed; Solid, unaltered bulk material; Slurry, water and sediment mixture; DI residue, remaining material after DI leaching; Clay, clay separates; Final residue, remaining material after nitric acid leaching; X, mineral is detected; I, illite; K, kaolinite; Se, sepiolite; Chl, chlorite; S, smectite/montmorillonite/beidellite; C, clinoptilolite; H, heulandite; M, mordentie; A, analcime; Z, unknown zeolite; P, plagioclase; K-spar, potassium feldspar; Y, yeelimite; Aph, aphthitalite; G, gaylussite; Ar, aragonite; $\mathrm{Mg}$, magnesite; Th, thermonatrite; $\mathrm{N}$, norsethite]

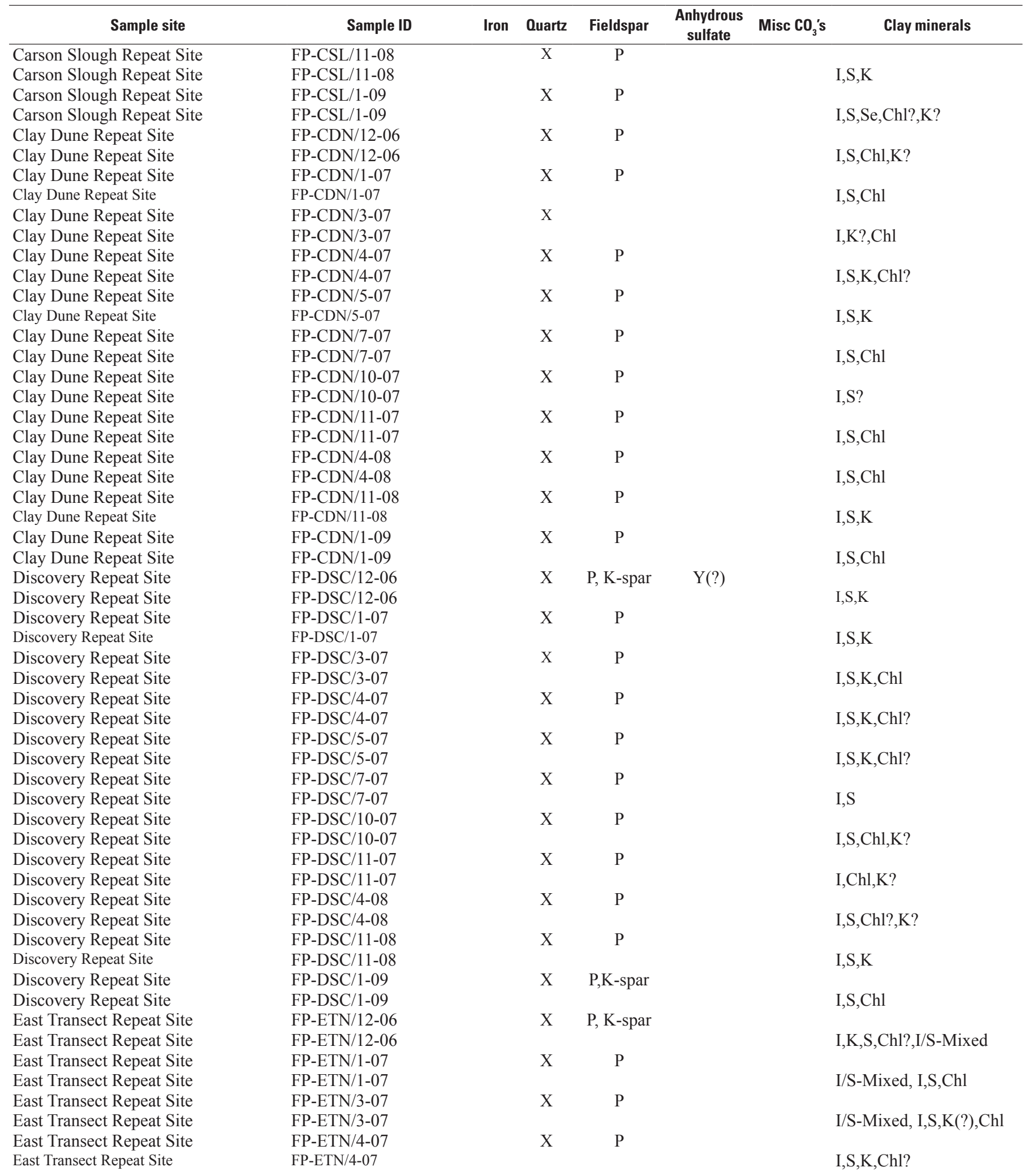


Table 15. Mineralogical data of auger hole and grab samples determined by X-ray diffraction.-Continued

[Sample site, name of sampling site; Sample ID, unique sample identifier; Treatment, type of sample preparation; Size, size fraction analyzed; Solid, unaltered bulk material; Slurry, water and sediment mixture; DI residue, remaining material after DI leaching; Clay, clay separates; Final residue, remaining material after nitric acid leaching; X, mineral is detected; I, illite; K, kaolinite; Se, sepiolite; Chl, chlorite; S, smectite/montmorillonite/beidellite; C, clinoptilolite; H, heulandite; M, mordentie; A, analcime; Z, unknown zeolite; P, plagioclase; K-spar, potassium feldspar; Y, yeelimite; Aph, aphthitalite; G, gaylussite; Ar, aragonite; Mg, magnesite; Th, thermonatrite; N, norsethite]

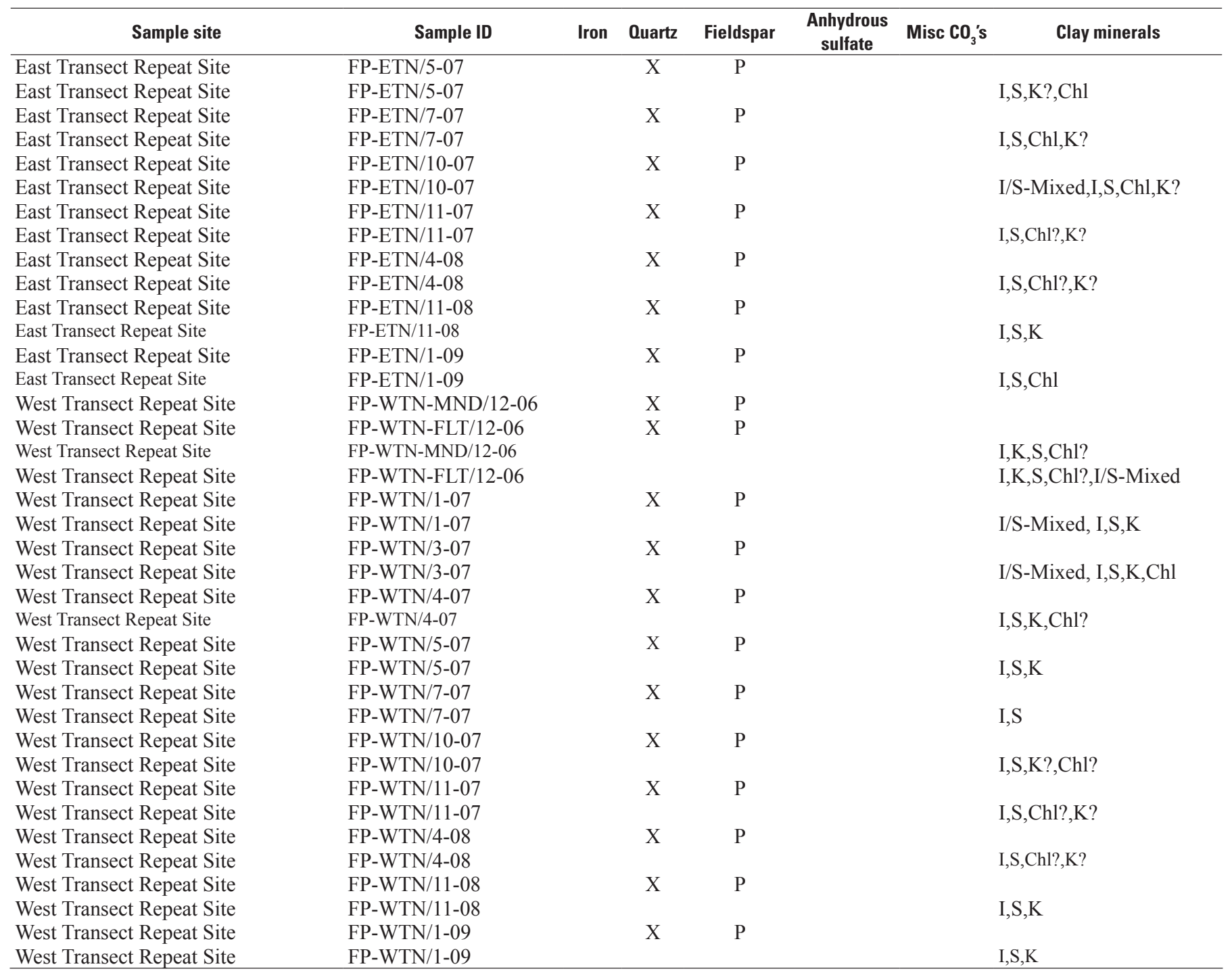

Publishing support provided by:

Denver Publishing Service Center, Denver, Colorado

For more information concerning this publication, contact:

Center Director, USGS Central Mineral and Environmental Resources

Science Center

Box 25046, Mail Stop 973

Denver, CO 80225

(303) 236-1562

Or visit the Central Mineral and Environmental Resources Science

Center Web site at:

http://minerals.cr.usgs.gov/

This publication is available online at:

http://pubs.usgs.gov/ds/607/ 


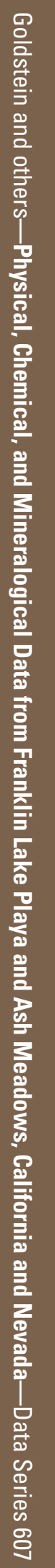

